Do gap junctions regulate synchrony in the parkinsonian basal ganglia?

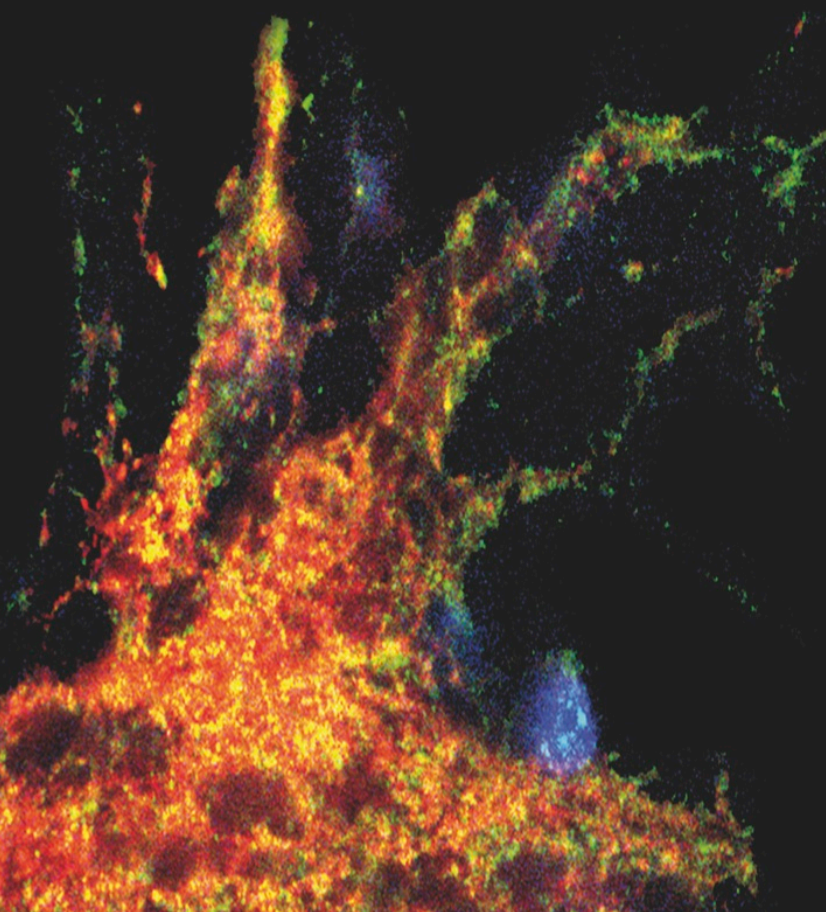

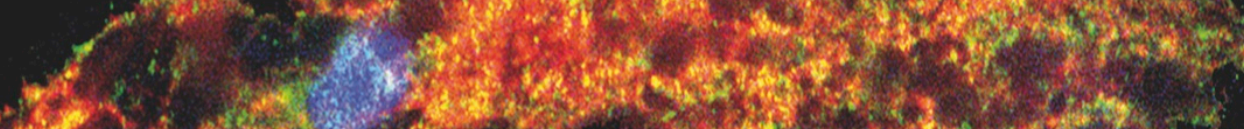

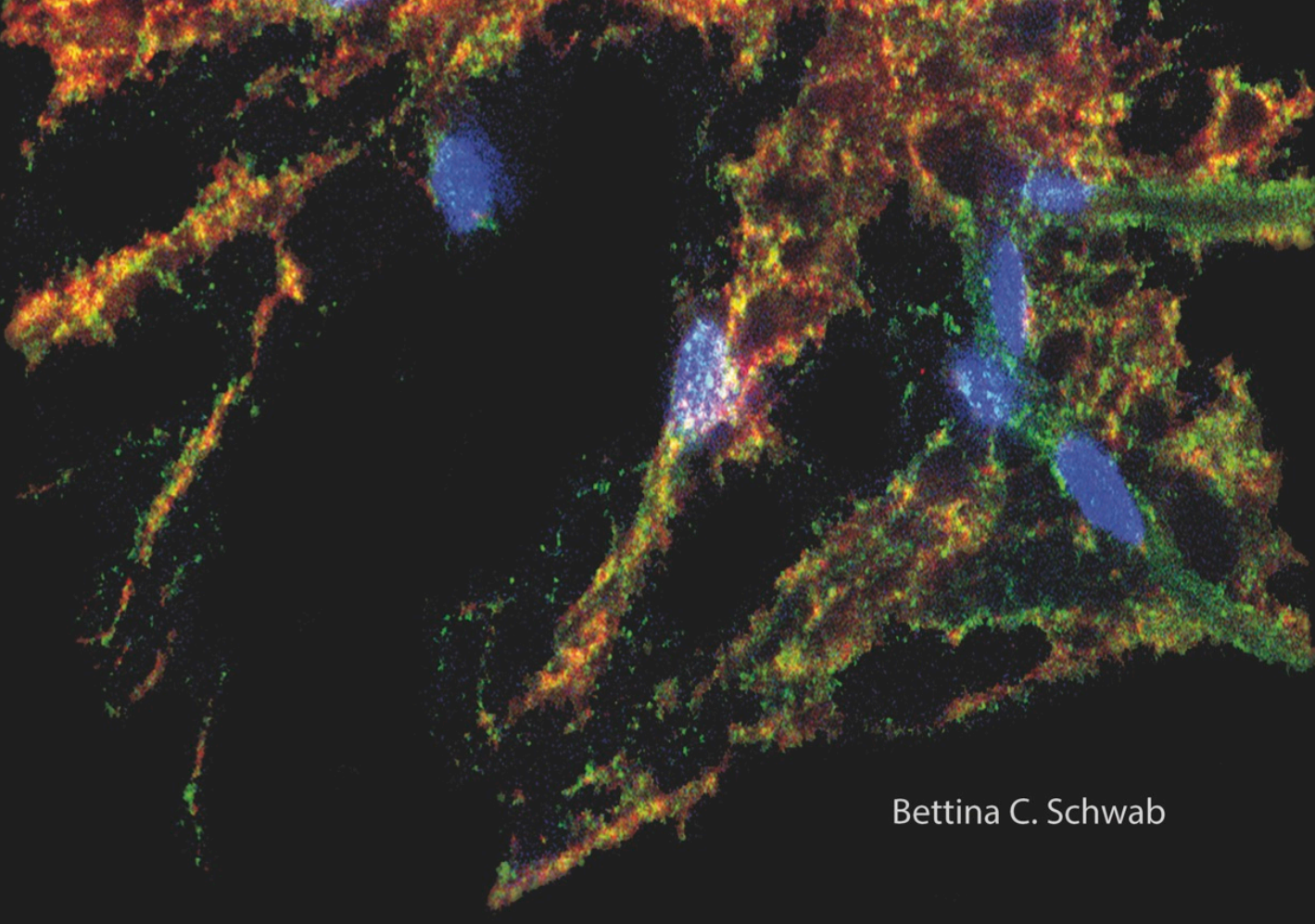




\title{
DO GAP JUNCTIONS REGULATE SYNCHRONY IN THE PARKINSONIAN BASAL GANGLIA?
}

\author{
Bettina C. Schwab
}


The research presented in this thesis was carried out in the groups Applied Analysis (AA) and Biomedical Signals and Systems (BSS), MIRA Institute for Biomedical Technology and Technical Medicine, University of Twente, PO Box 217, 7500 AE Enschede, The Netherlands, and the Department of Mathematics, University of Pittsburgh, PA, USA.

\section{MIRA}

BIOMEDICAL TECHNOLOGY

\section{UNIVERSITY OF TWENTE.}

The author acknowledges support by NDNS+ (Netherlands Organization for Scientific Research), the MIRA Institute for Biomedical Technology and Technical Medicine (University of Twente), and the German National Academic Foundation.

Schwab, Bettina C.

Do gap junctions regulate synchrony in the parkinsonian basal ganglia?

Ph.D. Thesis, University of Twente, 2016.

Copyright (C) 2016 by Bettina Schwab. All rights reserved.

Printed by Ipskamp Drukkers, Enschede, The Netherlands.

Cover: Connexin-36 (green) in the rat endopenduncular nucleus. (C) Bettina Schwab 


\title{
DO GAP JUNCTIONS REGULATE SYNCHRONY IN THE PARKINSONIAN BASAL GANGLIA?
}

\author{
PROEFSCHRIFT
}

ter verkrijging van

de graad van doctor aan de Universiteit Twente, op gezag van de rector magnificus, prof. dr. H. Brinksma, volgens besluit van het College voor Promoties

in het openbaar te verdedigen

op vrijdag 22 april 2016 om 16:45 uur

door

Bettina Christine Schwab

geboren op 19 maart 1988

te Nürtingen, Duitsland 
Dit proefschrift is goedgekeurd door de promotoren prof. dr. S.A. van Gils

en

prof. dr. R.J.A. van Wezel 
Samenstelling van de promotiecommissie:

Voorzitter en secretaris:

prof. dr. P.M.G. Apers Universiteit Twente

Promotoren:

prof. dr. S.A. van Gils Universiteit Twente

prof. dr. R.J.A. van Wezel Radboud Universiteit Nijmegen

Leden:

dr. L.J. Bour

Academisch Medisch Centrum Amsterdam

prof. dr. M.M.A.E. Claessens

Universiteit Twente

prof. dr. A.K. Engel

Universitätsklinikum Hamburg-Eppendorf

prof. dr. M.J.A.M. van Putten Universiteit Twente

prof. dr. J.E. Rubin

University of Pittsburgh

prof. dr. Y. Temel

Universiteit Maastricht 



\section{Contents}

1 Introduction

2 Synchrony in Parkinson's disease: Importance of intrinsic properties of

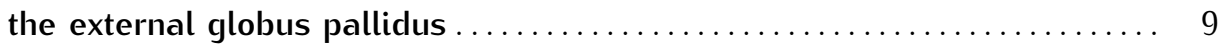

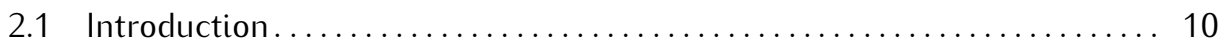

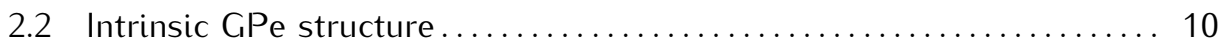

2.3 Important contribution of the GPe to the pathophysiology of

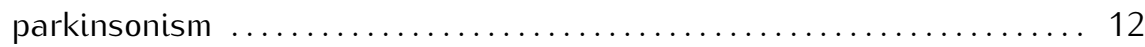

2.4 Potential intra-G.Pe mechanisms for (de)synchronization ........... 13

2.4.1 Cellular properties ............................... 13

2.4 .2 Synaptic properties .................................. 16

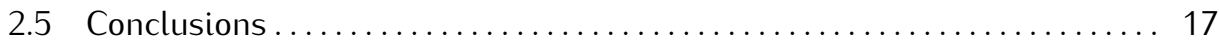

3 Existence of Connexin-36 in the human pallidum ................. 19

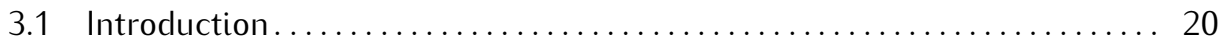

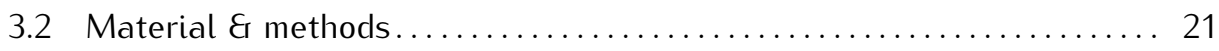

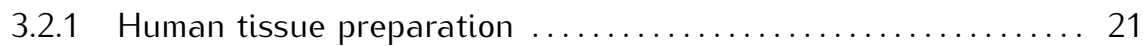

3.2.2 Fluorescent labeling and confocal imaging for $\mathrm{C} \times 36$ detection . 22

3.2.3 Quantification of the Cx36 signal .................. 24

3.2.4 Gap junctional coupling in a basic model of the basal ganglia . 24

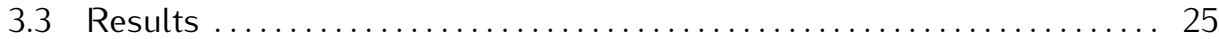

3.3.1 Cx36 was present in the human putamen, $\mathrm{C}_{1} \mathrm{Pe}$ and $\mathrm{C}_{\mathrm{P}} \mathrm{P}$, but not in the STN................................. 25

3.3.2 Rat control tissue also showed $\mathrm{C} \times 36 \ldots \ldots \ldots \ldots \ldots \ldots . \ldots . \ldots . \ldots 26$

3.3.3 Gap junctional coupling controls synchrony in a basic model

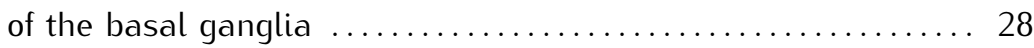

3.4 Discussion and conclusions ............................ 29 
4 Sparse pallidal connectivity shapes synchrony in a network model of

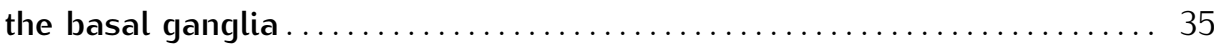

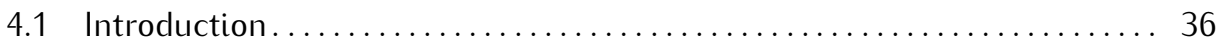

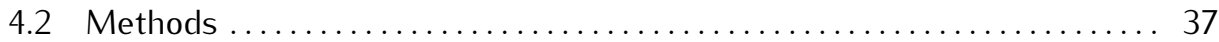

4.2.1 Basal ganglia network model .................... 37

4.2 .2 Neuron models ......................................... 39

4.2 .3 Quantification of synchrony ....................... 43

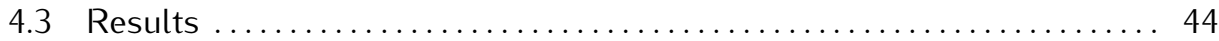

4.3.1 Estimated connectivity within the external part of the globus pallidus ...................................... 44

4.3.2 Lateral inhibition in C.Pe desynchronized the basal ganglia, whereas strong gap junction coupling synchronized......... 45

4.3.3 High gap junction coupling increased beta coherence........ 45

4.3.4 Effects already occurred in small model.................. 47

4.3.5 Similar results for Fujita neuron .................... 51

4.4 Discussion and Conclusions ................................. 51

5 Dynamics of the basal ganglia-thalamic connection during movement ... 55

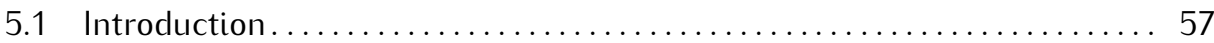

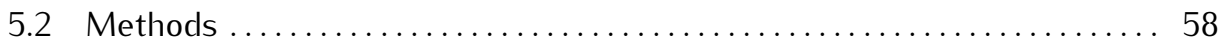

5.2.1 Experimental setup and data preprocessing .............. 58

5.2 .2 Verification of functional circuitry .................. 59

5.2 .3 Noise correlations .................................... 60

5.2.4 Detection of movement-related discharge .............. 60

5.2 .5 Spike and LFP correlations ........................ 61

5.2.6 Prediction of LFP correlations by a third LFP signal ....... 61

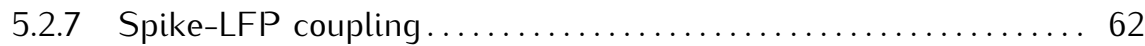

5.3 Results .............................................. 64

5.3.1 Basic properties of recorded activity . . . . . . . . . . . . 65

5.3.2 Low noise correlations between C.Pi and VLa .............. 65

5.3.3 Both $\mathrm{C}_{1} \mathrm{Pi}$ and VLa showed movement-related discharge ...... 69

5.3.4 Spike correlations between $C_{1} P i$ and VLa were absent or weak and not modulated during movement............. 72

5.3.5 LFP correlations between C.Pi and VLa were strong, and could only scarcely directly be explained by cortical input.... 74

5.3.6 Spike-LFP correlations could not confirm a clear feed-forward structure of the GiPi-VLa connection .................. 77

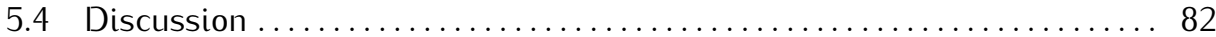


References .................................................. 101

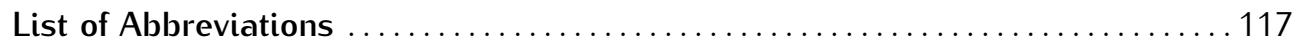

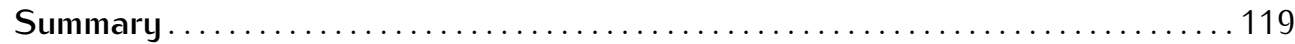

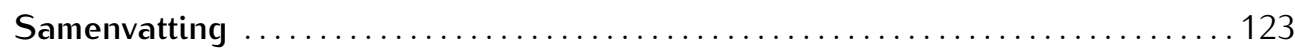

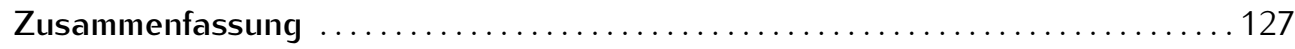

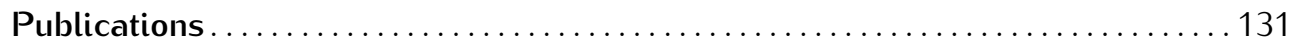

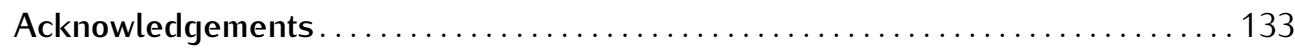





\section{Introduction}

"[...] It's like you're in the middle of the street and you're stuck there in cement shoes and you know a bus is coming at you, but you don't know when. [...]" 1

Although Parkinson's disease (PD) is often not fatal, patients suffer substantially from various symptoms, leading to high restrictions in daily life. These include motor symptoms such as involuntary rhythmic movements of the hands (tremor), stiffness, slowness of movement (bradykinesia) or even the inability to initiate movements (akinesia) (see Fig. 1.1), but also cognitive decline and psychological disorders. Typically, virtually all of those symptoms are experienced as debilitating and despite extensive research, a cure for Parkinson's disease is missing so far. However, many patients would appreciate treatments that would give relief to the symptoms, although the disease itself remains present. Up to now, treatments do exist, but their effectiveness is highly variable and often decreases over time. For example, medication replacing depleted dopamine in the brain (L-3,4-dihydroxyphenylalanine, L-Dopa) can reduce motor symptoms in initial stages of the disease, but often loses its impact after some years of treatment. In some patients, deep brain stimulation (DBS) is then used as an additional treatment. Here, an electrode has to be placed in the basal ganglia, usually in the subthalamic nucleus (STN), stimulating the surrounding tissue at high frequencies of around $130 \mathrm{~Hz}$. Not all patients show an improvement of motor symptoms after DBS, and many of them report neuropsychiatric or other side-effects. Thus, there is a strong need for improved and more gentle treatments of PD, not only targeting the initial stages of neural loss in the substantia nigra pars compacta ( $\mathrm{SNc}$ ), but also with respect to later stages and the commencement of motor symptoms. Now already, the occurrence of PD worldwide is huge, and as life expectancy is growing, even more people will suffer from this disease in the future. Basic research

\footnotetext{
${ }^{1}$ Quote, Interview with Michael J. Fox in "Good Houskeeping", May 2011, on living with Parkinson's disease.
} 
on the mechanisms underlying PD symptoms is needed to find better treatments.

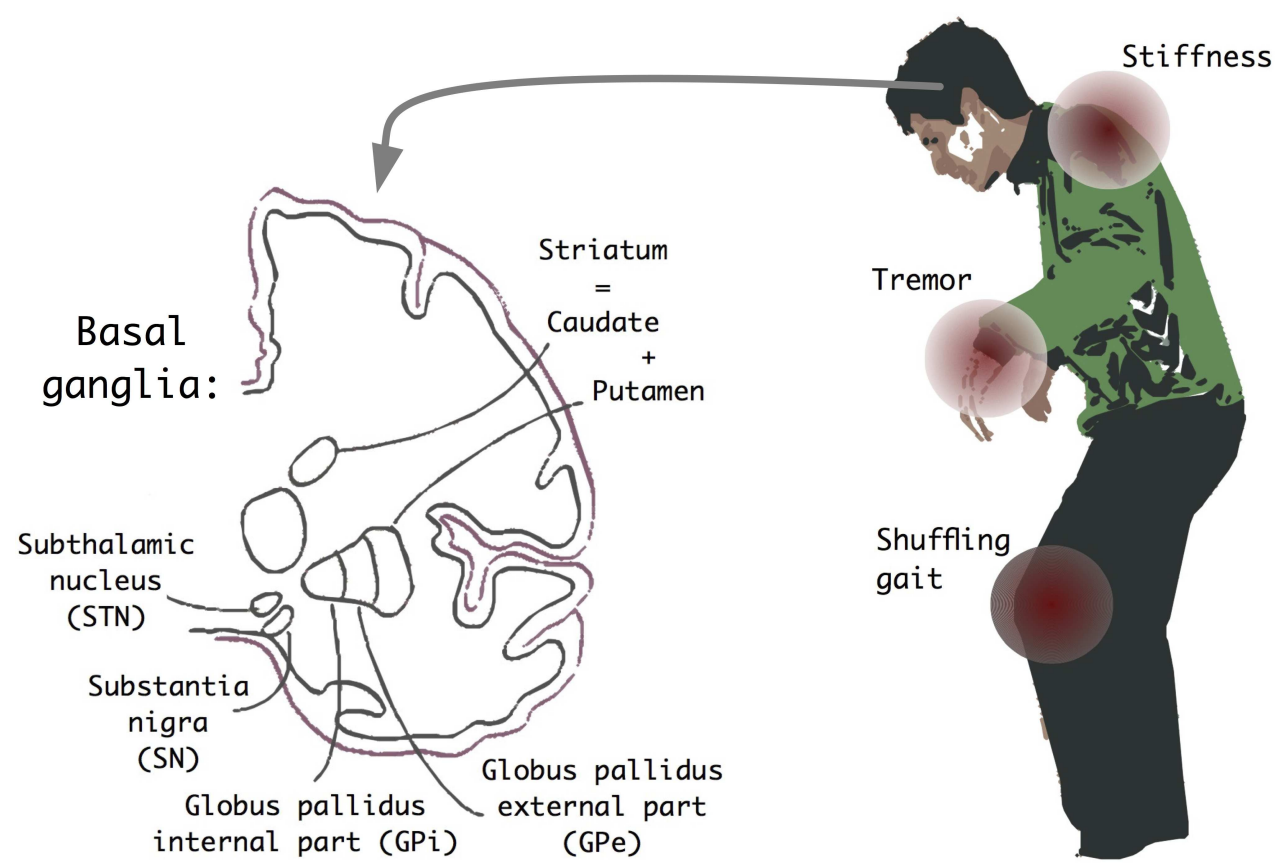

Figure 1.1: Motor symptoms in Parkinson's disease (PD) correlate with neural activity in the basal ganglia.

In this thesis, we look for possible mechanisms why PD patients exhibit motor symptoms, building the basis for future therapies. We introduce the idea that electrical coupling of neurons by gap junctions can influence activity and information processing in the basal ganglia, in particular after dopamine depletion, and thereby have a potentially high impact on movement control. In Chapter 2, we argue why the external part of the globus pallidus (CPe), having a central position in the basal ganglia, is our focus of attention. Especially, we explain how intrinsic properties of this nucleus could have a large influence on basal ganglia activity. Next, in Chapter 3, we seek experimental evidence for the existence of gap junctions within GPe. Further, we test the influence of pallidal gap junction and inhibitory coupling on activity in the basal ganglia in a computational model in Chapter 4. Finally, in Chapter 5, we aim to find out more about the relevance of basal ganglia activity for motor control, in particular of synchrony and oscillations, in recordings from monkeys performing a movement task. Although this thesis presents a storyline, and many lines of thought evolve over several chapters, it is without difficulty possible to read single chapters 
separately. First of all, we would like to introduce the main ideas here by asking some questions.

\section{Why does PD come along with motor symptoms?}

The severity of motor symptoms of PD is highly variable and can change within hours or sometimes even minutes. These symptoms often react well to L-Dopa and DBS, suggesting that not the direct neural loss is responsible for the deterioration of motor control, but that rather a dynamic process might be underlying the impairments. When dopaminergic cells in the SNc die, a lack of dopamine in the basal ganglia, in particular in striatum, emerges. Basal ganglia dopamine levels seem to be an important factor to preserve the healthy state of the basal ganglia: after severe dopamine depletion, motor symptoms occur. When PD patients take dopaminergic drugs such as L-Dopa, which increase the level of dopamine in the basal ganglia, motor symptoms are typically relieved, but not necessarily non-motor symptoms. However, not only dopamine seems to be important. Other neurotransmitters such as acetylcholine and neurotransmitter-independent processes are involved. Neural activity in the basal ganglia is thought to be crucial for precise and controlled movement. PD patients as well as animals after severe dopamine depletion show typical shifts in basal ganglia activities: more synchrony, low frequency oscillations, increased bursting and slight changes in firing rates (for review, see Galvan et al. [2015]). It has been proposed that altered activity in the basal ganglia leads to an inappropriate transmission of information to thalamus [Rubin et al., 2012, Anderson et al., 2015], finally leading to the inability to optimally control movements.

Most evidence for the importance of basal ganglia activity for impaired movement in PD is of correlative nature. Synchrony, low frequency oscillations and bursting correlate with motor symptoms, also when the symptoms are reduced by L-Dopa or DBS [Brown, 2003, Kühn et al., 2006, 2008, Galvan et al., 2015]. However, during the last years, it has become apparent that all those features are highly variable and task dependent - for example, beta oscillations might be dynamically modulated [Cagnan et al., 2015]. Thus, not only the presence of a certain type of basal ganglia activity alone could lead to motor symptoms, but especially its modulation during movement. Other possible factors contributing to motor symptoms might be competition between feedback loops [Leblois et al., 2006], reductions in movementrelated discharge [Rascol et al., 1992, Catalan et al., 1999, Turner et al., 2003], loss

of functional segregation within basal ganglia and cortex [Alexander et al., 1986, Filion et al., 1988, 1989, Boraud et al., 2000, Pessiglione et al., 2015] and abnormal 
timing of movement-related stimuli [Pasquereau et al., 2015]. Due to ample evidence for hypersynchrony in PD, we focus on abnormal synchrony in this thesis.

\section{Are connectivity changes the reason for altered basal ganglia activity?}

Many studies have targeted the question how basal ganglia activity shifts arise after dopamine depletion. Many of them assume or report a difference in connectivity within the basal ganglia (e. g., Terman et al. [2002], Miguelez et al. [2012], Fan et al. [2013], Gittis et al. [2011]) or from cortex to the basal ganglia [Magill et al., 2001, Deffains and Bergman, 2015, Mathai et al., 2015, DeLong and Wichmann, 2015, Chu et al., 2015]. It is unclear what connectivity changes are pathological, adaptive, maladaptive or epiphenomenological, and which connectivity changes occur only in animal models but not in patients. A lot of attention was given to the origin of lowfrequency oscillations: they might arise in cortex [Magill et al., 2001, Brown, 2003, Tachibana et al., 2011] or in the basal ganglia [Plenz and Kital, 1999, Terman et al., 2002, Tachibana et al., 2011] or by network effects involving cortex and basal ganglia [Pavlides et al., 2015]. Therefore, it seems crucial to study how the basal ganglia react on incoming synaptic currents from cortex. Also cellular properties of basal ganglia neurons can change and thereby define how the basal ganglia process incoming signals. We review both cellular and synaptic changes within the external part of the globus pallidus (GPe) in PD that could lead to the described activity shifts in Chapter 2. As the C.Pe has a very central position in the basal ganglia (see Fig. 1.2), it can have an organising and orchestrating role to define the level of synchrony in other basal ganglia nuclei.

In particular, we introduce a novel connectivity within GPe in Chapter 3: gap junctional coupling. Gap junctions are direct connections between cells that can lead to passive diffusion of electric charge and small particles. Often, gap junctions are associated with synchrony, but they can also induce desynchronization [Pfeuty et al., 2005, Vervaeke et al., 2010]. We describe how gap junctions in GPe might even be up-regulated in PD and can thereby shape synchrony in the basal ganglia. Notably, gap junctions in the retina change their conductance with the level of dopamine [Hampson et al., 1992, Li et al., 2013]. If pallidal gap junctions should have similar properties, regulation of gap junctional coupling by dopamine could be another way how dopamine influences basal ganglia activity. Phookan et al. [2015] had a similar hypothesis: they tested the effects of gap junction blockers, both in $\mathrm{C}_{1} \mathrm{Pe}$ and systemically, on basal ganglia activity. In particular, beta oscillations 
decreased after blocking gap junctions, which can be seen as a first confirmation of the importance of gap junctions for PD.

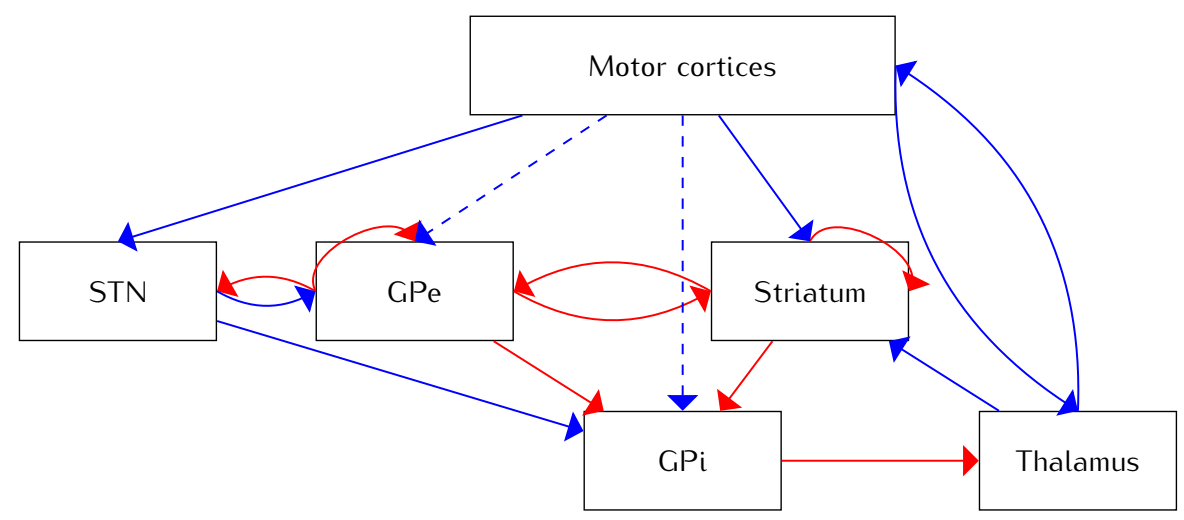

Figure 1.2: Scheme of macroscopic basal ganglia connectivity. Although the $\mathrm{C}_{1} \mathrm{Pe}$ is not an output nucleus that transmits information to thalamus, it has a very central position in the basal ganglia, projecting to all other basal ganglia nuclei and taking part in multiple feedback loops. Inhibitory pathways are shown in red, excitatory pathways in blue. Pathways with sparse evidence are depicted dashed.

\section{How can computational modeling help to understand those activity shifts?}

Mechanistic computational modeling can serve as an efficient tool to test the effect of connectivity changes on basal ganglia activity. Seeing a subset of the brain as a dynamic system, it can be described quantitatively, often yielding valuable insights into neural dynamics. Complementary to experiments, a lot of different settings can be described and guide further investigations. In contrast to descriptive or interpretive models, physiological and anatomical detail known from in-vivo and in-vitro studies is essential for mechanical models, and verification by further experiments is an important step. Such modeling has been done extensively for dynamics of basal ganglia activity related to Parkinson's disease. Early models [Terman et al., 2002, Rubin and Terman, 2004] described the system of STN and C.Pe as a pacemaker in the basal ganglia, leading to either uncorrelated or synchronized, bursting neural activity depending on connectivity, and studied down-stream effects of such activity on thalamus. Related models picked up those ideas and investigated different connectivity changes and their effects on activity (e. g., Kumar et al. [2011]). Later, 
when it became clear how important the intrinsic oscillatory nature of basal ganglia neurons is [Wilson, 2013], models using phase response curves (PRCs) became established (e. g., Schultheiss et al. [2010, 2012], Wilson et al. [2011], Holt and Netoff [2014]) which described the reactions of oscillating systems on inputs. Finally, in the last years, models of microcircuitries, for example in striatum [Gittis et al., 2011, Damodaran et al., 2015], have been investigated. Unfortunately, up to now, a lot of information on the mirco-architecture of basal ganglia connections is missing, impeding the development of such models.

In this thesis, we model the basal ganglia as a network of neurons (Chapter 4). Network models describe the dynamics of every single neuron explicitly, and can thereby be used to analyze synchrony between these neurons. We study the influence of pallidal inhibition and gap junction coupling on synchrony in the basal ganglia. Other types of models include for example neural mass or neural field models that lump the dynamics of single neurons and describe only average properties of neural populations. The latter models are therefore capable of describing large neural populations in tissue and their dynamics like oscillations.

\section{What is the (patho)physiological relevance of basal ganglia activity?}

As mentioned earlier, it is a critical question which activity changes in the basal ganglia are actually causal to PD symptoms, and, in which way basal ganglia activity influences movement in general. Tremor is often related to theta frequency oscillations (around $4-7 \mathrm{~Hz}$ ), and depression of this rhythm in motor cortex of PD patients by transcranial alternating current stimulation (tACS) was successful in reducing tremor [Brittain and Brown, 2013]. However, the situation seems to be more complicated for motor symptoms other than tremor, such as bradykinesia and stiffness. As one very popular hypothesis states that pathologically increased beta oscillations can lead to impaired movement (for review, see Brown [2003] and Engel and Fries [2010]), a number of studies used stimulation at beta frequency to test if there is indeed a causal relation. For example, Chen et al. [2003] stimulated the STN of PD patients via DBS electrodes at $20 \mathrm{~Hz}$, leading to moderate reductions in tapping rates. Pogosyan et al. [2009] used tACS at $20 \mathrm{~Hz}$ to drive cortical activity, which slightly reduced the velocity of voluntary movement compared to stimulation at $5 \mathrm{~Hz}$. However, reaction time did not differ and and the effects on movement were much lower than expected in both studies. Also direct stimulation of the rat STN at beta frequency using optogenetics did not lead to impaired movement, whereas stimula- 
tion of afferent fibers to STN did [Gradinaru et al., 2009]. Hence, the total power of beta oscillations might not be the sole critical factor, but details like, for example, timing of beta coherence could be essential.

Another way to relate basal ganglia activity changes to symptoms are models of progressive parkinsonism. Leblois et al. [2007] described that synchronized oscillations in the monkey pallidum appeared later than the parkinsonism when dopamine was depleted slowly over time, making a major influence of those oscillations on the symptoms unlikely. In a similar study using a rat model, Janssen et al. [2012] reported that bursting of neurons is present before motor symptoms appear, and might therefore be rather compensatory than causal to impaired movement. Finally, it is also possible to study the relevance of basal ganglia activity by looking at its downstream effects: if a certain feature of basal ganglia activity - say, increased synchrony - does not have any effect on downstream structures, it is unlikely to cause symptoms. With this approach, it is also possible to study effects of physiological basal ganglia activity, and how the basal ganglia exert their influence on thalamus and successively on motor cortex. In Chapter 5, we do this in monkeys: both basal ganglia output and thalamic input activity were recorded. Although we did not record from parkinsonian animals yet, the effects of heathy basal ganglia activity during movement could be studied. Chapter 5 is independent of the gap junction hypothesis presented earlier.

\section{Will we ever understand what our brain really does?}

It is not the aim of this thesis to find out how our mind works, or why we behave the way we behave. Indeed, we focus on tiny features, like gap junctions in the $\mathrm{C}_{1} \mathrm{Pe}$, and how these features change certain types of activity, like synchrony. The models used in this thesis include far too few neurons, or neural tissue, not enough realistic heterogeneity and other biological detail to reproduce actual dynamics in the brain. Still, by only looking at those little subsystems of the brain, we can already draw conclusions on how the brain should not work - or, in other words, what could make it sick. This is possible without understanding the full system, and opens possibilities for novel treatments. Nevertheless, much more - for example, the questions whether our brain is deterministic, why we are all so different, how neurons of mathematicians work - is left for important discussions after sunset. 



\title{
Synchrony in Parkinson's disease: Importance of intrinsic properties of the external globus pallidus
}

\begin{abstract}
The mechanisms for the emergence and transmission of synchronized oscillations in Parkinson's disease, which are potentially causal to motor deficits, remain debated. Aside from the motor cortex and the subthalamic nucleus, the external globus pallidus ( $\left.C_{1} P e\right)$ has been shown to be essential for the maintenance of these oscillations and plays a major role in sculpting neural network activity in the basal ganglia. While neural activity of the healthy C.Pe shows almost no correlations between pairs of neurons, prominent synchronization in the $\beta$ frequency band arises after dopamine depletion. Several studies have proposed that this shift is due to network interactions between the different basal ganglia nuclei, including the GPe. However, recent studies demonstrate an important role for the properties of neurons within the $C_{1} P e$ In this review, we will discuss these intrinsic GPe properties and review proposed mechanisms for activity decorrelation within the dopamine-intact C.Pe. Failure of the GPe to desynchronize correlated inputs can be a possible explanation for synchronization in the whole basal ganglia. Potential triggers of synchronization involve the enhancement of $\mathrm{C}_{1} \mathrm{Pe}-\mathrm{C}_{1} \mathrm{Pe}$ inhibition and changes in ion channel function in $\mathrm{C}_{1} \mathrm{Pe}$ neurons. ${ }^{1}$
\end{abstract}

\footnotetext{
1 Adapted from Schwab et al. [2013a], Frontiers in Systems Neuroscience, 7 (60).
} 


\subsection{Introduction}

Neural activity in the basal ganglia of patients with idiopathic Parkinson's disease (PD) and animal models of PD commonly shows high levels of synchronization, bursting and oscillations in low frequency bands such as $\theta(4-7 \mathrm{~Hz})$ and $\beta(15-30 \mathrm{~Hz})$ frequencies [Bergman et al., 1994, Obeso et al., 2000, Brown et al., 2001, Montgomery, 2007, Wichmann et al., 2011]. Although it is not completely clear whether these abnormal neural activities cause PD motor symptoms, they are reliable disease markers as they coincide with motor symptoms after severe dopamine depletion [Kühn et al., 2006, 2009, Hammond et al., 2007, Eusebio et al., 2012, Quiroga-Varela et al., 2013]. Nevertheless, the mechanisms and origins of the emergence and transmission of synchronization, bursting and oscillations remain controversial. Oscillations in the $\beta$ frequency range, often related to rigidity, akinesia and bradykinesia, have been proposed to arise via the cortex [Brown, 2003, Sharott et al., 2005, Tachibana et al., 2011] or via interactions of the subthalamic nucleus (STN) and the external globus pallidus (G.Pe) [Plenz and Kital, 1999, Bevan et al., 2002, Terman et al., 2002, Tachibana et al., 2011, Fan et al., 2013].

After dopamine depletion, prominent changes in neural synchronization occur in projection neurons of the $\mathrm{C}_{1} \mathrm{Pe}$, which has a central position in the basal ganglia loop [Smith et al., 1998] $]^{2}$. Under healthy conditions, activity in the G.Pe shows almost no correlations between pairs of neurons [Nini et al., 1995, Raz et al., 2000, Mallet et al., 2008], including spatially nearby neurons [Bar-Gad et al., 2003], although neurons in the G.Pe possess a large number of local axon collaterals and are believed to receive common inputs [Francois et al., 1984, Percheron et al., 1991, Yelnik, 2002]. In contrast, after dopamine depletion, strong synchronization in the $\beta$ frequency range was found [Nini et al., 1995, Raz et al., 2000, Heimer et al., 2002, Mallet et al., 2008]. These findings led to the suggestion of a local mechanism that decorrelates activity in the healthy GPe [Bar-Gad et al., 2003]. Failure of the C.Pe to decorrelate synchronized input can be an explanation for abnormal synchrony of the whole basal ganglia.

In this review, we discuss recent evidence supporting the crucial role of GPe properties in synchronizing and desynchronizing afferent activity and their remodeling in Parkinsonism. We describe proposed mechanisms for this synchronization process intrinsic to the G.Pe, based on synaptic and cellular properties.

\footnotetext{
2 The primate external and internal globus pallidus (GPe and $\mathrm{G}_{\mathrm{P}} \mathrm{Pi}$ ) are named globus pallidus (G,P) and endopenduncular nucleus (EP), respectively, in rodents. We will refer to $C_{1} P e$ and $C_{1} P i$ for these nuclei in general.
} 


\subsection{Intrinsic G.Pe structure}

The G.Pe is located centrally in the basal ganglia and contributes to its multiple feedback loops [Jaeger and Kita, 2011]. Its neural dynamics, involving high firing rates, are strongly influenced by excitatory inputs from the STN [Goldberg et al., 2003]. $C_{1} A B A e r g i c$ synapses projecting to the CPe are provided mainly by the striatum and about $10 \%$ of the synapses arise in the GPe itself [Shink and Smith, 1995]. Morphological characterization of these local axon collaterals in the rat brain indicates that the G.P not only acts as a relay nucleus, but has intrinsic structures capable of internal information processing [Sadek et al., 2007]. In these structures, information is processed from neurons in the outer part of the GP to neurons in the inner part [Sadek et al., 2007]. This elaborate C.P internal connectivity seems essential for sculpting GP activity, and C,P projection neurons may take additional roles as inhibitory interneurons that control spiking behavior.

In healthy awake animals, two electrophysiological cell types have been identified in the GPe based on their firing rates and patterns [deLong, 1971, Bugaysen et al., 2010, Benhamou et al., 2012]. 6-hydroxydopamine (6-OHDA) treated rats also showed clear differences in the firing rates and patterns between two distinct C.P neuron populations in vivo [Mallet et al., 2008]. In contrast, studies using healthy rat brain slices described three electrophysiological subgroups of neurons in the C.P [Cooper and Stanford, 2000, Bugaysen et al., 2010]. However, other in vitro studies reported no clear qualitative electrophysiological differences amongst GP neurons and challenge the existence of distinct G.P neuron types [Chan et al., 2004, Hashimoto and Kita, 2006, Giünay et al., 2008, Chan et al., 2011, Deister et al., 2013].

Nevertheless, anatomical dichotomy has often been described in the C.P [Hoover and Marshall, 2002, Cooper and Stanford, 2002, Nobrega-Pereira et al., 2010]. For instance, a group of proenkephalin positive neurons that preferentially target the striatum [Hoover and Marshall, 2002] and a small population of calretinin positive interneurons [Cooper and Stanford, 2002] have been reported. Based on fate mapping analysis, even five neural populations have been identified in the mouse GP which differ in progenitor lineage and partly in their embryonic domains [Nobrega-Pereira et al., 2010].

Recently, Mallet et al. [2012] combined anatomical and electrophysiological characteristics of classes of G.P neurons. They described the existence of two distinct neural populations in the GP of a 6-OHDA treated rat that have distinct molecular profiles and axonal connectivities. Neurons of the first population fired antiphasic to STN neurons, often expressed parvalbumin (PV) and targeted the STN or the 
EP. The second population described a novel cell type: neurons that fired in-phase with STN neurons expressed proenkephalin and innervated both projection neurons and interneurons of the striatum. Mallet et al. [2012] also found differences in the dendritic and axonal architectures of the two cell types. In particular, local axon collaterals of the first neural population were longer while their dendritic spine density was lower in comparison to the second population.

Altogether, the complex structure of the GPe on both the synaptic and cellular levels indicates that information processing within the $\mathrm{C}_{1} \mathrm{Pe}$ is possible and might be critical for modulating dynamics in the whole basal ganglia network. Studies using rodents have extensively described different groups of cells within the G.P, but published evidence for different GPe cell types in higher species is lacking. Combinations of electrophysiology and anatomy, as done for rodents in Mallet et al. [2012], will be needed to evaluate whether cell differences are also of importance in primates, and to clarify their role for information processing in the basal ganglia.

\subsection{Important contribution of the G.Pe to the pathophysiology of parkinsonism}

The GPe is in a unique position to propagate and orchestrate synchronized oscillatory activity, since it projects to virtually all other basal ganglia nuclei [Mallet et al., 2008]. Furthermore, its neurons possess intrinsic oscillatory properties, leading to a steady pacemaking function [Wilson, 2013]. Nevertheless, $\beta$ band oscillations in the $\mathrm{C}_{1} \mathrm{Pe}$ in Parkinsonism commonly exhibit smaller amplitudes than those in the STN or the GPi [Stein and Bar-Gad, 2013]. An important hypothesis proposes that the GPe plays a major role in information processing in the dopamine depleted basal ganglia, in particular by interacting with the STN [Plenz and Kital, 1999, Bevan et al., 2002, Terman et al., 2002, Fan et al., 2013]. A study in 1-Methyl-4-Phenyl-1,2,3,6Tetrahydropyridine (MPTP) treated monkeys showed that muscimol inactivation of the $\mathrm{G}_{1} \mathrm{Pe}$ to block its $\mathrm{C}_{1} \mathrm{ABAergic}$ outputs led to prominent reductions of $\beta$ oscillations in the STN [Tachibana et al., 2011]. The C.Pe is therefore assumed to regulate the presence of oscillations in the dopamine depleted basal ganglia, while the origins of these oscillations remain unclear.

Due to the central position within the basal ganglia and its potential to orchestrate basal ganglia activity, the GPe may be a natural target for deep brain stimulation. Although C.Pi and especially STN are virtually the only sites where deep brain stimulation for patients with $\mathrm{PD}$ is applied in common clinical practice, some 
studies have evaluated the C.Pe as a target [Vitek et al., 2004, Angeli et al., 2015]. While deep brain stimulation of $\mathrm{C}_{1} P$ e in general led to an improvement of akinesia, rigidity and tremor, initially even in comparison to $\mathrm{C}_{\mathrm{P}} \mathrm{Pi}$ stimulation, the improvement declined over the subsequent weeks [Angeli et al., 2015]. Given the detailed intrinsic structure of $\mathrm{G}_{1} \mathrm{Pe}$, it is possible that adaptive processes like synaptic plasticity occurred during prolonged stimulation, altering the clinical effect of deep brain stimulation. As Angeli et al. [2015] speculated, deep brain stimulation in GPe might thus particularly profit from an adaptive stimulation type such as closed-loop stimulation.

Since neural activity abnormalities in PD are at least partly reversible with L3,4-dihydroxyphenylalanine (L-Dopa) treatment, their emergence and reversal are both thought to be crucially dependent on dopamine levels [Brown et al., 2001, Kühn et al., 2006, Tachibana et al., 2011]. However, it is not clear yet how dopamine acts on the basal ganglia and whether it reverses the parkinsonian activity to the original. Despite the fact that in literature the effects of dopamine depletion are often focused on the striatum, PD patients lose about $82 \%$ of the original dopamine levels in the G.Pe [Rajput et al., 2008]. This specific loss is also linked to motor symptoms as supported by several studies investigating the influence of dopamine directly in the rat C.P. Firstly, dopamine receptor $D_{1} / D_{2}$ blockage in the $C_{1} P$ induced akinesia [Hauber and Lutz, 1999]. Secondly, direct application of dopamine in the G.P restored motor behaviour in a 6-OHDA model [Galvan et al., 2001]. Thirdly, injections of 6OHDA in the $\mathrm{G}_{1} \mathrm{P}$ induced dopamine depletion in both $\mathrm{G}_{1} \mathrm{P}$ and striatum and mimicked the parkinsonian motor symptoms and neural activity abnormalities resulting from striatal 6-OHDA injections [Abedi et al., 2013].

These findings support the important role of dopamine depletion in the GPe for PD. Furthermore, the results of Abedi et al. [2013] additionally indicate that direct injury of the GPe could contribute to PD pathology. Indeed, Fernandez-Suarez et al. [2012] reported prominent cell death of PV-positive $C_{1} A B A$ Aergic $G_{1} P e$ neurons, commonly projecting to STN and $\mathrm{C}_{\mathrm{P}} \mathrm{Pi}$, in 6-OHDA treated rats and in MPTP treated monkeys. In contrast, an earlier study by Hardman and Halliday [1999] did not describe abnormalities in the total number of PV-positive GPe neurons in PD patients. However, when considering cell density rather than absolute cell counts, death of C.Pe neurons is also possible here as seen in a trend towards a decrease of PVpositive neuron density [Fernandez-Suarez et al., 2012]. Fernandez-Suarez et al. [2012] speculate that a loss of $C_{1} A B A$ Aergic $C_{1} P_{e}$ neurons could decrease inhibition of the STN and thus support its hyperactivity. Furthermore, the C.Pe may lose parts of its intrinsic structure, thereby forfeiting its ability to perform complex information 
processing. To prevent secondary cell death, adaptive processes could be triggered that may additionally impede information processing.

\subsection{Potential intra-C.Pe mechanisms for (de)synchronization}

Several mechanisms have been proposed for increased synchronization inside the $\mathrm{C}_{1} \mathrm{Pe}$ in Parkinsonism or, in turn, for desynchronisation of this nucleus under healthy conditions. The majority of these mechanisms are based on interactions between the C.Pe and other nuclei, namely the STN and striatum [Alexander and Crutcher, 1990, Ingham et al., 1997, Plenz and Kital, 1999, Terman et al., 2002, Kumar et al., 2011, Fan et al., 2013]. In the following sections, we describe intrinsic C.Pe mechanisms for (de)synchronization, involving cellular and synaptic C.Pe properties.

\subsubsection{Cellular properties}

Intrinsic properties of $\mathrm{C}_{1} \mathrm{Pe}$ neurons are determined by more than 10 voltage-gated ion channel types [Mercer et al., 2007, Günay et al., 2008, Jaeger and Kita, 2011]. Changes in the expression or function of these channels can contribute to changes in activity dynamics and influence synchrony in vivo. Hyperpolarization and cyclic nucleotide-gated (HCN), small conductance calcium-activated potassium (SK) and fast, transient, voltage-dependent sodium ( $\mathrm{NaF}$ ) channels as well as cellular heterogeneity in general have been proposed to desynchronize the dopamine intact G.Pe.

\subsubsection{HCN channel expression}

HCN channels, permeable to both sodium and potassium, are activated by hyperpolarization of the membrane and stay open at voltages near the resting membrane potential [Benarroch, 2013]. They are widely expressed in the dendrites of neurons in various parts of the brain such as the cortex, hippocampus and thalamus [Poolos, 2012]. They support pacemaking and can take part in sculpting synaptic responses [Chan et al., 2004]. Chan et al. [2004] proposed HCN channels in G.P neuron dendrites as key determinants of regular spiking and synchronization. In a study of HCN channel function in 6-OHDA lesioned mice, Chan et al. [2011] uncovered an HCN channelopathy in C.P neurons that accompanied pacemaking deficits. HCN channels 
located presynaptically on GP terminals are known to decrease the likelihood of CABA release [Boyes and Bolam, 2007]. Viral delivery of HCN subunits and L-type calcium channel agonists restored pacemaking, but did not improve motor symptoms, suggesting that the channelopathy might therefore be an adaptive process and not causal for motor deficiency [Chan et al., 2011].

\subsubsection{SK channel expression}

Activated by increases in the intracellular calcium concentration, SK channels lead to a low-conductance potassium current [Adelman et al., 2012]. These channels are assumed to contribute to the firing dynamics in most excitable cells [Bond et al., 1999] and can modulate plasticity [Woodward et al., 2010]. Studies with brain slices of healthy rats [Deister et al., 2009] and computational models of G.Pe neurons [Deister et al., 2009, Schultheiss et al., 2010] proposed a mechanism of decorrelation via an SK current. Deister et al. [2009] showed that rat C.P neurons express functional SK channels that contribute to the precision of autonomous firing in GP neurons, and strong SK currents can decrease the sensitivity of GPe neurons to smaller synchronized inputs [Deister et al., 2009]. Phase response curve analysis suggested that dendritic SK channel expression controls synchronization by changing the phase dependence of synaptic effects on spike timing [Schultheiss et al., 2010]. Further, SK channels can indirectly be modulated via dopamine [Ramanathan et al., 2008] and may therefore exhibit altered dynamics in PD.

\subsubsection{NaF channel expression}

Some dendritic voltage-dependent channels can open very fast and lead to transient sodium currents after membrane depolarization. The resulting sodium influx can further depolarize the membrane and induce an action potential. Hence, the initiation and propagation of action potentials on dendrites significantly depends on these NaF channels [Hanson et al., 2004]. High expression of dendritic NaF channels has been suggested as a potential mechanism that actively decorrelates the $\mathrm{C}_{1} \mathrm{Pe}$ [Edgerton and Jaeger, 2011]. In their computational model, Edgerton and Jaeger [2011] showed that neurons with low dendritic NaF channel expression have a high tendency to phase lock with synchronized synaptic input. They estimated that SK channel expression is only relevant in synchronizing neural activity if the dendritic $\mathrm{NaF}$ channel conductance is low compared to the conductance of other channels. 
Additionally, Edgerton and Jaeger [2011] report that HCN channel expression did not significantly alter oscillatory firing, leaving dendritic $\mathrm{NaF}$ channel expression as the main factor in determining the phase-locking properties of neurons. GP neurons of rats express dendritic $\mathrm{NaF}$ channels and their distribution is enriched near sites of excitatory synaptic input [Hanson et al., 2004]. Whether dendritic NaF channel expression actually decreases in PD has not been investigated yet. However, in other neuron types, it has been reported that $\mathrm{NaF}$ current density is subject to regulation through multiple pathways and on multiple timescales [Herzog et al., 2003, Hu et al., 2005, Xu et al., 2005], for example by dopamine $\mathrm{D}_{2}$ receptor-activated $\mathrm{Ca}^{2+}$ signaling within few minutes [Hu et al., 2005].

\subsubsection{Cellular heterogeneity}

Recently, Deister et al. [2013] suggested cellular heterogeneity as an active decorrelation mechanism. They found that the heterogeneity in firing rates and patterns found in G.P neurons in healthy rats are not due to multiple cell types or synaptic transmission but rather caused by a change over time in cellular properties common to all neurons, leading to different cellular characteristics within minutes. Quantitative changes in the expression of $\mathrm{HCN}$ or other ion channels could underly this dynamic cellular heterogeneity. Continuous variations in ion channel composition could account for the entire range of firing rates and patterns in the GPe [Günay et al., 2008]. Since neurons firing at widely different rates do not tend to synchronize with each other, this cellular heterogeneity may make the G.Pe less susceptible to synchronized inputs. Deister et al. [2013] therefore describe a powerful mechanism of decorrelation in the healthy C.Pe. However, changes in this heterogeneity after dopamine depletion have not yet been investigated.

In summary, in the dopamine intact basal ganglia, HCN, SK and NaF channels as well as cellular heterogeneity have been convincingly argued to contribute to neural dynamics in the G.Pe. A qualified hypothesis states that $C_{1} P e$ neurons are not very dependent on synaptic input due to their intrinsic pacemaker function, potentially sustained by HCN and SK channel function [Wilson, 2013]. Loss of the autonomous $\mathrm{C}$ Pe activity could lead to correlation of neural activity by shared inputs. However, cellular changes in the GPe after dopamine depletion that could cause such a loss are rarely studied in experiments. It therefore seems likely that cellular properties contribute to desynchronization of the healthy G.Pe, but it remains unclear whether these properties induce synchronization after dopamine depletion. 


\subsubsection{Synaptic properties}

Synaptic coupling inside the C.Pe via local axon collaterals is well established [Francois et al., 1984, Kita, 1994, Sadek et al., 2007, Miguelez et al., 2012] although functional C.Pe connectivity is highly variable and depends on the brain state [Magill et al., 2006]. Rat G.P-G.P synapses have considerably different properties than striatum-G,P synapses, with a lower paired-pulse ratio and weak responses to stimulation [Sims et al., 2008]. Although little is known about the effects of GABAergic transmission within the $\mathrm{C}_{1} \mathrm{Pe}$, connections from the GPe to the STN and the substantia nigra pars reticulata $(\mathrm{SNr})$ are better described and may share characteristics of $\mathrm{C}_{1} \mathrm{Pe}-\mathrm{G}_{1} \mathrm{Pe}$ connections. In rat brain slices, G.P-STN connections have been found to be sparse, but sufficiently powerful to inhibit and synchronize the autonomous activity of STN neurons [Baufreton et al., 2009]. Bursts of activity from the rat G.P are also able to effectively silence the firing of $\mathrm{SNr}$ neurons, although they can start firing again due to depression of these G.P-SNr synapses [Connelly et al., 2010]. A recent study demonstrates that rat G.P-G.P connections are also highly efficacious in modulating postsynaptic activity despite substantial short time depression and sparse connectivity [Bugaysen et al., 2013].

\subsubsection{Synaptic strength}

Miguelez et al. [2012] showed that G.P-G,P inhibitory synaptic transmission increased in a rat 6-OHDA model, leading to enhanced rebound bursting. This altered transition may have major impacts on neural dynamics. Kita et al. [2004, 2006] demonstrated that specific blocking of $C_{1} A B A$ receptors in the monkey $C_{1} P e$ regularizes neuron firing, indicating that $C_{1} A B A$ ergic inhibition from the striatum and $C_{1} P e$ regulates palliclal firing. It is still unclear how much and which influence inhibitory $\mathrm{C}_{1} \mathrm{Pe}-\mathrm{C}_{1} \mathrm{Pe}$ coupling has on synchrony in Parkinsonism. Coupling between GiPe cells could either synchronize or desynchronize activity [Wilson, 2013]. In the healthy GiPe, given the pacemaking function of these neurons, local axon collaterals may act as desynchronizing elements [Sims et al., 2008, Wilson, 2013]. However, after dopamine depletion, the effect of local axon collaterals could be reversed and synchronize activity [Wilson, 2013]. 


\subsubsection{Synaptic architecture}

Highly heterogeneous synaptic coupling between C.Pe neurons can also be a factor for their desynchronization. As heterogeneity on a cellular basis can act as a decorrelator, highly inhomogeneous coupling amongst neurons could lead to similar effects. Sadek et al. [2007] described the anatomical network of C.P-C.P axon collaterals in the rat as structured rather than homogeneously distributed. It can be speculated that through injury or adaptive remodeling, this structure may become damaged and lose the ability to desynchronize.

Although changes in synaptic transmission within the rat G.P after dopamine depletion have been measured [Miguelez et al., 2012], the detailed intrinsic connectivity of GPe still remains poorly understood. Nevertheless, it has become evident that this nucleus cannot only be considered as a homogeneous relay nucleus [Sadek et al., 2007]. Further studies of its structural and functional connectivity, especially at different dopamine levels, are needed to shed light on information processing inside the GPe.

\subsection{Conclusions}

Several lines of evidence emphasize the importance of intrinsic C.Pe properties in abnormal synchronization in Parkinsonism. This makes the GPe an attractive target for future therapies, potentially involving direct pharmacological targeting.

Most of the evidence provided in this review is based on rodent studies, but the rodent G.P may differ substantially from the human G.Pe in some aspects. Functionally, a lower average firing rate has been observed in the rodent C.P compared to the primate GPe, while firing patterns were very similar [Benhamou et al., 2012]. Anatomically, little is known about the level of human GPe local collateralization, although its existence is hardly debated [Francois et al., 1984]. The rat G.P is studied in more detail and shows a high level of complex local connections [Sadek et al., 2005, 2007].

Though often assumed, it remains unclear whether increased synchronization in the basal ganglia causes motor impairments in PD patients [Quiroga-Varela et al., 2013]. The onset of the synchronization process occurs independently of the onset of motor symptoms in animal models of increasing levels of dopamine depletion [Leblois et al., 2007, Dejean et al., 2012]. Nevertheless, impact of $\beta$ band synchro- 
nization on motor control remains a common assumption [Brittain and Brown, 2013]. A comprehensive mechanism responsible for synchronization and desynchronization of the GPe, that is dependent on dopamine levels, is still missing. However, loss of pacemaker function in $C_{1} P e$ neurons and altered function of G Pe-G.Pe synapses are important candidates [Wilson, 2013].

Although this review focuses on intrinsic GPe properties, we do not suggest that interactions in the basal ganglia network are less important. Synaptic input to the $\mathrm{C}_{1} \mathrm{Pe}$, mainly from STN and striatum, plays a major role in pallidal synchronization [Goldberg et al., 2003, Tachibana et al., 2011]. We propose that intrinsic mechanisms of the GPe are crucial in processing these synchronized or partly synchronized inputs, thereby determining dynamics of feedback loops to STN or striatum.

After dopamine depletion, G.P neurons undergo plastic changes in their synaptic and cellular structure [Chan et al., 2011, Miguelez et al., 2012, Wichmann and Smith, 2013], which may potentially trigger synchronized neural activity. However, further studies on ion channel remodeling after dopamine depletion and their effects on synchrony and motor performance are missing. Intrinsic G.Pe connectivity is still insufficiently described and may not be restricted to GABAergic transmission. We emphasize that special attention should be drawn to possible cell death in the $\mathrm{C}_{\mathrm{Pe}}$ [Fernandez-Suarez et al., 2012]. Adaptive processes could be triggered to prevent further cell death that may lead to altered neural activity, which might involve synaptic as well as cellular changes. 



\title{
Existence of Connexin-36 in the human pallidum
}

\begin{abstract}
Background: While increased synchrony of the neural activity in the basal ganglia may underlie the motor deficiencies exhibited in Parkinson's disease (PD), it remains unknown how this synchrony arises, propagates through the basal ganglia and changes under dopamine replacement. Gap junctions could play a major role in modifying this synchrony, as they show functional plasticity under the influence of dopamine and after neural injury.

Methods: Confocal imaging was used to detect connexin-36, the major neural gap junction protein, in post-mortem tissues of PD patients and control subjects in the putamen, subthalamic nucleus (STN) and external and internal globus pallidus (GPe and $\mathrm{C}_{1} \mathrm{Pi}$, respectively). We quantified how gap junctions affect synchrony in an existing computational model of the basal ganglia.

Results: We detected connexin-36 in the human putamen, GPe and C.Pi, but not in the STN. Furthermore, we found that the number of connexin-36 spots in PD tissues increased by $50 \%$ in the putamen, $43 \%$ in the C.Pe and $109 \%$ in the C.Pi compared to controls. In the computational model, gap junctions in the C.Pe and C.Pi strongly influenced synchrony. The basal ganglia became especially susceptible to synchronize with input from the cortex when gap junctions were numerous and high in conductance.

Conclusions: Connexin-36 expression in the human $\mathrm{C}_{1} \mathrm{Pe}$ and $\mathrm{C}_{1} \mathrm{Pi}$ suggests that gap junctional coupling exists within these nuclei. In PD, neural injury and clopamine depletion could increase this coupling. Therefore, we propose that gap junctions act as a powerful modulator of synchrony in the basal ganglia. ${ }^{1}$
\end{abstract}

\footnotetext{
1 Adapted from Schwab et al. [2014], Movement Disorders, 29 (12), pp. 1486-1494.
} 


\subsection{Introduction}

In the basal ganglia of patients with Parkinson's disease (PD) and corresponding animal models, unusual high amounts of synchrony, bursting and low frequency oscillations have been recorded [Wichmann et al., 2006]. These abnormalities are thought to underlie the motor symptoms of PD, but it remains unclear whether they are causal [Quiroga-Varela et al., 2013]. Still, the mechanisms for the emergence and transmission of synchrony and oscillations in the basal ganglia remain debated. Experimental and computational studies have shown that interactions between the subthalamic nucleus (STN) and the external segment of the globus pallidus (GPe) are important for the emergence of synchrony [Plenz and Kital, 1999, Terman et al., 2002, Bevan et al., 2002, Fan et al., 2013]. Other studies highlighted the role of synaptic input from the cortex to the STN [Magill et al., 2001, Brown, 2003, Sharott et al., 2005, Hahn and Mclntyre, 2010, Tachibana et al., 2011].

Changes in the intrinsic properties of the C.Pe can also lead to synchrony [Chan et al., 2011, Miguelez et al., 2012, Wilson, 2013, Schwab et al., 2013a]. While the healthy GPe shows almost no correlations between pairs of neurons despite the presence of local axon collaterals and correlated inputs [Nini et al., 1995, Bar-Gad et al., 2003], synchronization in the $\beta$ frequency band $(13-30 \mathrm{~Hz})$ is prominent after dopamine loss [Nini et al., 1995, Raz et al., 2000, Heimer et al., 2002]. Therefore, it has been suggested that a decorrelation mechanism exists in the healthy C.Pe [BarGad et al., 2003]. Given the pacemaking function of G.Pe neurons, intra-G.Pe synaptic coupling may play an important role in synchronization and desynchronization in the C.Pe. However, few experimental studies have described changes in the GPe after dopamine loss that would explain the clear shifts in network dynamics seen in PD. Furthermore, it remains unclear how these mechanisms may change under dopamine replacement.

Pallidal gap junctions may provide an intrinsic mechanism of synchronization. It has already been proposed that gap junctional coupling in cortex and striatum contributes to the pathology of PD [Yamawaki et al., 2008, Traub and Whittington, 2010, Weinberger and Dostrovsky, 2011, Dere, 2012, Damodaran et al., 2014]. Crap junctions between interneurons of striatum and cortex consist of connexin-36 (Cx36) [Galarreta and Hestrin, 2001, Fukuda, 2009], which has also been found in the rat globus pallidus (C.P), corresponding to the human C.Pe [Rash et al., 2000]. Various other neurological pathologies are thought to involve altered gap junction coupling, including epilepsy, stroke, spreading depression and ischemia [Nemani et al., 2005, Wang et al., 2010, Bargiotas et al., 2012], all of which involve neural injury which is in general asso- 
ciated with remodeling of gap junctions [Belousov et al., 2012]. Fernandez-Suarez et al. [2012] reported the death of parvalbumin (PV)-positive CABAergic neurons in the $\mathrm{C}_{\mathrm{P}} \mathrm{P}$ of animal models of $\mathrm{PD}$, raising the possibility of gap junction remodeling.

Dopamine can also influence gap junctional coupling: for example, gap junctions in the retina change their conductance in response to variations in the dopamine level [McHahon et al., 1989, Hampson et al., 1992, Li et al., 2013]. The majority of studies on gap junctions in the brain found a decrease in gap junction conductance with increased dopamine levels [Traub and Whittington, 2010]. Dye coupling, an indication for gap junction coupling, was increased in the striatum after dopamine loss in rats [Cepeda et al., 1989, Onn and Crace, 1999]. Dopamine modulation of gap junction coupling in the striatum has also been associated with stereotypic behavior [Moore and Cirace, 2002], emphasizing the potential impact of gap junction coupling on clinical characteristics. Although the presence of gap junctions in the human $\mathrm{C}_{1} \mathrm{Pe}$, $\mathrm{C}_{1} \mathrm{Pi}$ and STN would significantly impact information processing in the basal ganglia, it remains unknown whether they exist and how they may remodel after clopamine depletion.

In this study, we therefore studied Cx36 expression in the putamen, G.Pe, GPi and STN of post-mortem tissues from PD patients and control subjects. We furthermore incorporated gap junctions into a basic conductance-based computational model of the basal ganglia to examine their potential influence on synchrony. Based on our findings, we hypothesize that gap junctions exist between CABAergic neurons of the $\mathrm{C}_{1} \mathrm{Pe}$ and $\mathrm{C}_{\mathrm{Pi}} \mathrm{a}$ and suggest that they undergo redistribution due to neural injury in $\mathrm{PD}$ and exhibit up-regulated conductances after dopamine depletion. The existence of numerous high-conductance gap junctions in the C.Pe may diminish the ability of pallidal neurons to desynchronize correlated input.

\subsection{Material $\varepsilon$ r methods}

\subsubsection{Human tissue preparation}

Human tissue was obtained from The Netherlands Brain Bank (NBB), Netherlands Institute for Neuroscience, Amsterdam. All material was collected from donors for or from whom written informed consent for a brain autopsy and the use of the material and clinical information for research purposes had been obtained by the NBB. One control subject that showed a local bacterial proliferation in the basal ganglia was excluded (not shown in Table 3.1). All patients were male and between 71 and 
96 years of age. The control and PD patient groups were matched in age $(82 \pm 11$ years) and post-mortem delay $(5.1 \pm 1.4 \mathrm{~h})$. Based on their clinical information, the control subjects did not suffer from any neurological disease. Quantitative scores on the severity of motor symptoms in the PD patients were not available.

The formalin-fixed, paraffin-embedded tissues were deparaffinized using xylene and ethanol. Biopsies were taken from putamen (part of the striatum), $\mathrm{G}_{1} \mathrm{Pe}$ and $\mathrm{C}_{1} \mathrm{Pi}$ (6 PD patients and 6 control subjects as described in Table 3.1) and STN (2 PD patients and 2 control subjects, partly coinciding with the previous group). Tissues were immersed in $25 \%$ sucrose for at least 48 hours prior to being frozen to prevent tissue damage. Frozen biopsies were then sectioned using a cryostat along the coronal plane at a thickness of $60 \mu \mathrm{m}$.

\subsubsection{Fluorescent labeling and confocal imaging for Cx36 detection}

We used triple labeling to image $C \times 36$ on CABAergic neurons. Free-floating sections were first permeabilized and blocked with phosphate buffered saline containing $0.5 \%$ Triton-X-100 and 10\% goat serum. Next, sections were incubated in primary and secondary antibodies for approximately 24h and $8 \mathrm{~h}$, respectively. 1:300 dilutions of mouse monoclonal IgC1 anti-Cx36 (Invitrogen) and rabbit polyclonal Ig $C_{1}$ anti-C,AD65/67 (Sigma) were used to detect gap junctions and CABAergic neurons, respectively. 4',6-diamidino-2-phenylindole (DAPI) was applied in a 1:500 dilution to label the cell nuclei. The secondary antibodies were conjugated to Alexa Fluor 488 and 633 (Invitrogen).

To reduce lipofuscin-like autofluorescence, which was in particular present our human tissue, we applied an autofluorescence eliminator reagent (Millipore) for 10 min. The samples were then rinsed in ethanol and mounted on glass slides with Fluoromont-G (Electron Microscopy Sciences). Imaging was performed on a Nikon A1 confocal microscope with a 100x oil lens. To avoid bleed-through, we sequentially scanned the specimens with individual lasers. A minimum of 20 images per tissue group was taken, with a resolution of $1024 \times 1024$ pixels or $0.124 \mu \mathrm{m}$ in both directions. 
Table 3.1: List of patients from whom tissue of putamen, GiPe and GiPi has been analyzed.

\begin{tabular}{|c|c|c|c|c|c|c|c|c|c|c|c|c|}
\hline Patient \# & 1 & 2 & 3 & 4 & 5 & 6 & 7 & 8 & 9 & 10 & 11 & 12 \\
\hline PD & no & no & no & no & no & no & 11 years & 8 years & 4 years & 3 years & 21 years & 10 years \\
\hline $\begin{array}{l}\text { Cause of } \\
\text { Death }\end{array}$ & \begin{tabular}{|c|} 
heart \\
failure
\end{tabular} & \begin{tabular}{|c|} 
bronchus \\
carcinoma
\end{tabular} & \begin{tabular}{|c|} 
myocardial \\
infarction
\end{tabular} & sepsis & $\begin{array}{l}\text { cardiac } \\
\text { asthma }\end{array}$ & $\begin{array}{c}\text { urinary tract } \\
\text { infection }\end{array}$ & \begin{tabular}{|c|} 
metabolic \\
de-regulation
\end{tabular} & \begin{tabular}{|c|} 
heart \\
failure
\end{tabular} & \begin{tabular}{|l|} 
respiratory \\
insufficiency
\end{tabular} & $\begin{array}{l}\text { respiratory } \\
\text { insufficiency }\end{array}$ & $\begin{array}{c}\text { de- } \\
\text { hydration }\end{array}$ & euthanasia \\
\hline Age & 96 & 74 & 87 & 71 & 85 & \begin{tabular}{|c|}
96 \\
\end{tabular} & 87 & \begin{tabular}{|l|}
84 \\
\end{tabular} & \begin{tabular}{|l|}
83 \\
\end{tabular} & \begin{tabular}{|l|}
87 \\
\end{tabular} & 74 & 81 \\
\hline $\begin{array}{c}\text { Post-Mortem } \\
\text { Delay }\end{array}$ & \begin{tabular}{|c|}
$6 \mathrm{~h}$ \\
$30 \mathrm{~min}$
\end{tabular} & $5 \mathrm{~h}$ & $4 \mathrm{~h}$ & \begin{tabular}{|c|}
$7 \mathrm{~h}$ \\
$40 \mathrm{~min}$
\end{tabular} & \begin{tabular}{|c|}
$4 \mathrm{~h}$ \\
$15 \mathrm{~min}$
\end{tabular} & $\begin{array}{c}4 \mathrm{~h} \\
10 \mathrm{~min}\end{array}$ & $\begin{array}{c}4 \mathrm{~h} \\
45 \mathrm{~min}\end{array}$ & $\begin{array}{c}5 \mathrm{~h} \\
10 \mathrm{~min}\end{array}$ & $\begin{array}{c}5 \mathrm{~h} \\
45 \mathrm{~min}\end{array}$ & $\begin{array}{c}7 \mathrm{~h} \\
20 \mathrm{~min}\end{array}$ & $\begin{array}{c}2 \mathrm{~h} \\
50 \mathrm{~min}\end{array}$ & $\begin{array}{c}4 \mathrm{~h} \\
30 \mathrm{~min}\end{array}$ \\
\hline Tremor & \begin{tabular}{|c|} 
no \\
\end{tabular} & no & no & no & no & no & yes & yes & no & yes & yes & yes \\
\hline Stiffness & no & no & no & no & no & no & yes & no & yes & yes & no & yes \\
\hline $\begin{array}{l}\text { Brady-I } \\
\text { Akinesia }\end{array}$ & no & no & no & no & no & no & yes & yes & yes & yes & yes & yes \\
\hline $\begin{array}{c}\text { L-Dopa } \\
\text { Medication } \\
\end{array}$ & no & no & no & no & no & no & $\begin{array}{l}\text { not during } \\
\text { last year }\end{array}$ & yes & yes & yes & no & yes \\
\hline \begin{tabular}{|c|} 
Cx36 Putamen \\
$\left(\right.$ spots $\left./ \mathrm{mm}^{2}\right)$
\end{tabular} & 1770 & 830 & 1266 & 1171 & 1187 & 788 & 2332 & 2159 & 1723 & 865 & 1475 & 1939 \\
\hline \begin{tabular}{|c|} 
Cx36 Putamen \\
(spot area/\%)
\end{tabular} & 0.022 & 0.018 & 0.028 & 0.019 & 0.026 & 0.017 & 0.039 & 0.024 & 0.026 & 0.016 & 0.038 & 0.037 \\
\hline $\begin{array}{c}\mathrm{C} \times 36 \mathrm{C} \text { Pe } \\
\left(\text { spots } / \mathrm{mm}^{2}\right)\end{array}$ & 1266 & 902 & 1001 & 371 & 919 & 1083 & 1299 & 1462 & 1580 & 711 & 1254 & 1611 \\
\hline $\begin{array}{c}\text { Cx36 C.Pe } \\
\text { (spot area/\%) }\end{array}$ & 0.018 & 0.013 & 0.021 & 0.008 & 0.019 & 0.025 & 0.025 & 0.017 & 0.026 & 0.011 & 0.029 & 0.038 \\
\hline $\begin{array}{c}\mathrm{C} \times 36 \mathrm{C} \mathrm{CPi} \\
\left(\text { spots } / \mathrm{mm}^{2}\right)\end{array}$ & 1059 & 556 & 712 & 771 & 709 & 779 & 2165 & 2204 & 1109 & 1955 & 940 & 1220 \\
\hline $\begin{array}{c}\mathrm{C} \times 36 \mathrm{C} \mathrm{CPi} \\
\text { (spot area/\%) }\end{array}$ & 0.016 & 0.008 & 0.013 & 0.012 & 0.018 & 0.015 & 0.038 & 0.025 & 0.017 & 0.025 & 0.023 & 0.033 \\
\hline
\end{tabular}

We show clinical information and detected Cx36 levels for individual subjects and patients.

Occurrence of the PD symptoms tremor, stiffness and brady- or akinesia were described based on the clinical reports.

The symptoms were assumed to be absent if not mentioned in the report. 
The tissue selection process was not blinded. However, we tried to minimize the effects of unblinded sampling by selecting tissue areas solely based on the DAPI signal. Our Cx36 antibody has been tested in Cx36-knockout mice [Bautista et al., 2014]. Only very slight background staining was present in this negative control (Bautista et al. [2014], Fig. S1, supporting information). As the antibody itself has been raised in mouse, we expect that this background staining is even decreased in our rat or human tissue.

\subsubsection{Quantification of the Cx36 signal}

Confocal micrographs were analyzed using ImageJ [Rasband, 1997-2012]. We used a threshold segmentation approach to quantitatively estimate the level of Cx36 expression: assuming that the image intensity histogram is a bimodal distribution, the threshold was defined as the arithmetic mean of $p_{1}$, the peak intensity of the background noise, and and $p_{2}$, the highest signal intensity:

$$
t=\frac{\left(p_{1}+p_{2}\right)}{2}
$$

Images without a bimodal intensity distribution or with bright unspecific staining were rejected. In the segmented image, only spots with an area between 4 and 40 pixels were considered. In this way, segmentation of noise and unspecific labeling was suppressed.

\subsubsection{Gap junctional coupling in a basic model of the basal ganglia}

Depending on their architecture and strength, gap junctions can be both synchronizing or desynchronising [Chow and Kopell, 2000, Lewis and Rinzel, 2003, Vervaeke et al., 2010] and can interact in a nonlinear way with inhibitory synapses [Pfeuty et al., 2005]. Computational modelling can be used to study how a correlated input from cortex to STN affects synchronization, and how synchrony is spread to other nuclei. We implemented the network model proposed by Rubin and Terman [2004] including 16 cells to represent each of STN, G.Pe and G.Pi using MATLAB [Mathworks, 2012]. As shown in Fig 1a, the STN received excitatory input from the cortex, both $\mathrm{C}_{1} \mathrm{Pe}$ and $\mathrm{C}_{1} \mathrm{Pi}$ received inhibitory input from the striatum. We added gap junctions between pairs of neurons inside the $C_{1} P e$ and $C_{1} P i$. The neural dynamics in the $C_{1} P e$ and $C_{1} P i$ were thus governed by: 


$$
C_{m} \frac{d V}{d t}=-l_{\text {ionic }}-I_{\text {syn }}-I_{G J}+I_{a p p}
$$

where $C_{m}$ is the membrane capacity, $V$ the transmembrane voltage and $I_{\text {ionic }}, I_{\text {syn }}$, $I_{G J}$ and $I_{\text {app }}$ the ionic, synaptic, gap junction and applied currents, respectively. Gap junctions were modelled as ohmic resistors:

$$
I_{G J}=g_{G J} \cdot \Delta V
$$

with gap junction conductance $g_{C_{1}}$. $\Delta V$ represents the difference in transmembrane voltage between the connecting cells.

We chose two different gap junction coupling architectures (Fig $1 \mathrm{~b}$ ) to estimate the effect of newly synthesized gap junction channels: (1) sparse coupling with an average of 0.5 gap junctions per cell and (2) numerous coupling with an average of 1 gap junction per cell. To simulate dopamine modulation of the gap junction strength, the gap junction conductance in the $C_{1} P e\left(g_{G_{1} P e}\right)$ and $C_{1} P i\left(g_{G_{1}} P_{i}\right)$ was varied between 0 and $0.25 \frac{\mathrm{mS}}{\mathrm{cm}^{2}}$, a realistic range for neural gap junctions [Fortier, 2010], but low compared to the conductances of chemical synapses. The STN received excitatory input from the cortex in the form of white noise, either completely correlated or completely uncorrelated. The inhibitory input from the striatum to the G.Pe and C.Pi was uncorrelated white noise.

To quantify synchrony, we performed principal component analysis (PCA) on spike activity as described in Lourens et al. [2015]. In short, we calculated the number of principal components (PCs) needed to describe 95\% of the information contained in the spike times for all 16 cells in each nucleus. High synchronization is associated with a small number of PCs, indicating that little variation is needed to describe the network state.

\subsection{Results}

\subsubsection{Cx36 was present in the human putamen, GPe and GPi, but not in the STN}

STN tissues from neither PD patients nor control subjects showed significant levels of Cx36 (data not shown) and were thus excluded from further analysis. However, we found a clear punctuate pattern of $C_{x} 36$ labeling in the putamen, C.Pe and C.Pi of all subjects (Fig. 3.2), which was absent in a negative control without the primary anti- 


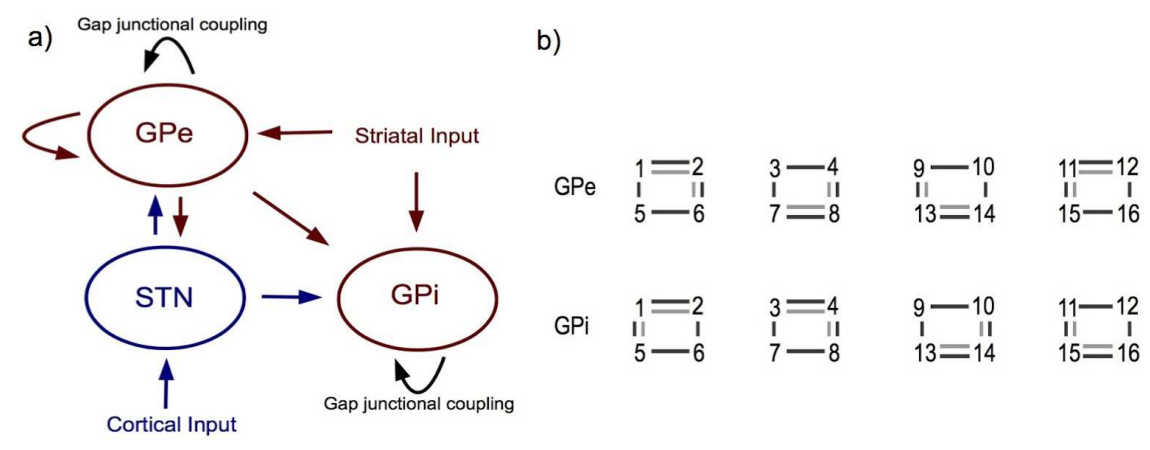

Figure 3.1: Placement of gap junctions added to the Rubin-Terman model [Rubin and Terman, 2004]. (a) General setup of STN, G.Pe, G.Pi and inputs from striatum and cortex. Red indicates inhibitory connections, blue excitatory connections, black gap junction coupling. (b) Gap junction architectures in the C.Pe and C.Pi. Numbers represent the 16 cells in both nuclei, connected in groups of four via gap junctions and in the GPe also via inhibitory synapses (not shown). Light grey lines indicate the architecture for sparse gap junction coupling, dark grey lines the architecture for numerous gap junction coupling.

body (data not shown). Table 3.1 summarizes the clinical background of all subjects and the results of the $\mathrm{C} \times 36$ quantification. An average of 18, but at least 12 images per tissue group could be included for image analysis.

Gap junction coupling in the putamen is well described and thought to be increased after dopamine depletion [Cepeda et al., 1989, Onn and Grace, 1999]. We therefore used $\mathrm{C} \times 36$ expression in the putamen as a reference for numerous gap junction coupling. Control subjects showed the most $\mathrm{C} \times 36$ expression in the putamen; less $\mathrm{C} \times 36$ was found in their $\mathrm{C}_{1} \mathrm{Pe}$ and $\mathrm{CP}$. Compared to controls, the number of Cx36 spots in PD patients increased by 50\% in the putamen, $43 \%$ in the $\mathrm{C}_{1} \mathrm{Pe}$ and $109 \%$ in the $\mathrm{CIPi}$ (Fig. 3.3(a)). Furthermore, the cumulative area of the Cx36 spots increased significantly in the putamen and $\mathrm{C}_{1} \mathrm{Pi}$ of $\mathrm{PD}$ patients, but no significant increase was detected in the GPe (Fig. 3.3(b)).

\subsubsection{Rat control tissue also showed CX36}

The labelling and tissue quality of our human tissue was restricted due to a postmortem delay of several hours and formalin fixation. We therefore applied the same labelling to rat tissue slices that had been fixed in 4\% paraformaldehyde for $15 \mathrm{~min}$. 

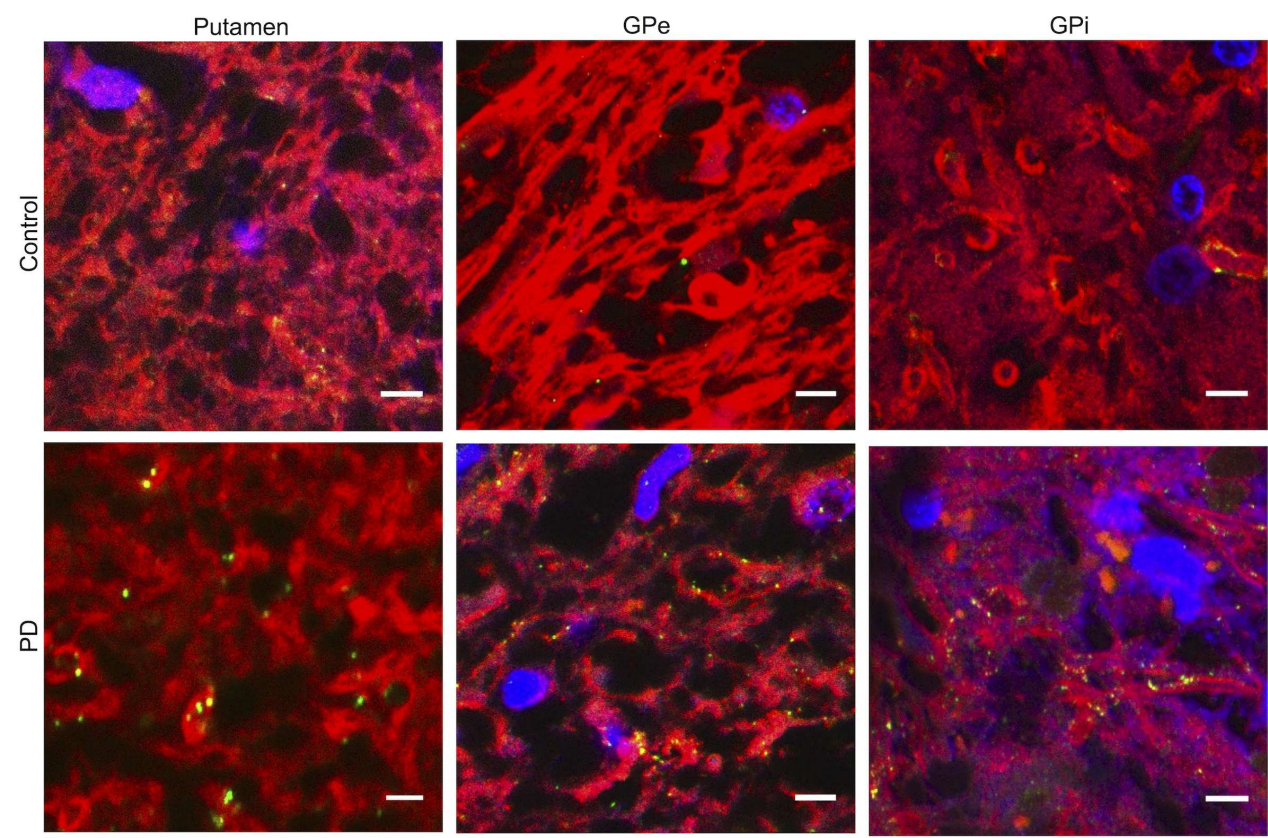

GAD67/65 Cx36 DAPI

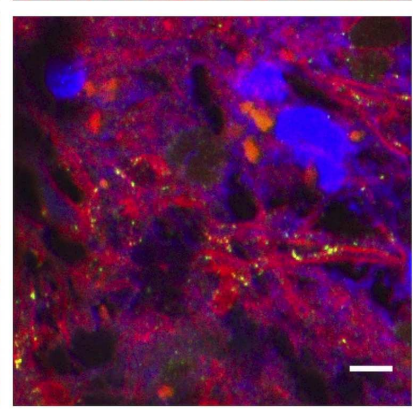

Scale bar: $5 \mu \mathrm{m}$

Figure 3.2: Cx36 in the human basal ganglia: Small high-resolution outtakes from confocal images. Cell nuclei are labelled by DAPI (blue), GABAergic neurons by anti-CAD65/67 (red) and Cx36 by anti-Cx36 (green). Some Cx36 is present in the putamen, $\mathrm{G}_{1} \mathrm{Pe}$ and $\mathrm{CP}$ i of control subjects, while an increase of $\mathrm{C} \times 36$ can be seen in the PD patients.

a)

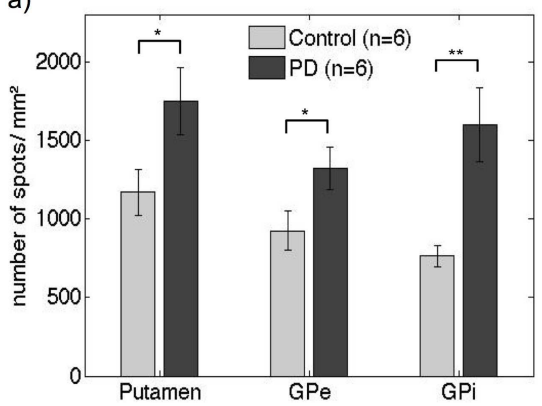

b)

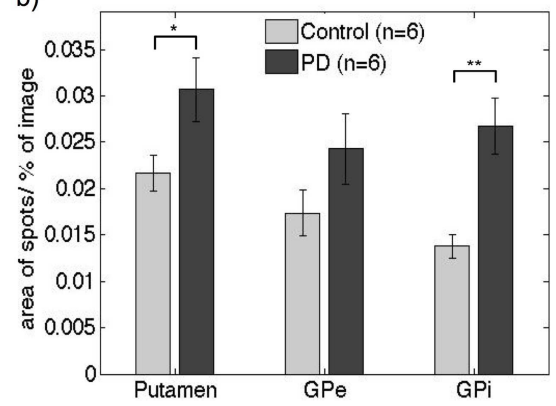

Figure 3.3: Average expression of $\mathrm{C} \times 36$ : (a) number of spots per $\mathrm{mm}^{2}$; (b) total area of the spots as a fraction of the total image. Cx36 spots are significantly $(p<0.05)$ increased in all three nuclei. The increase in cumulative area of detected Cx36 spots is significant only in putamen and $\mathrm{C}_{1} \mathrm{Pi}$. The standard errors of the mean are presented as thin bars. " $p<0.05$; $\mathrm{p} p<0.01$ (two sample t-test) 
Images of the rat globus pallidus (C,P, analog of the human $\left.C_{1} P e\right)$ and endopenduncular nucleus (EP, analog of the human $\left.C_{1} P i\right)$ clearly showed the existence of $C \times 36$ (Fig. 3.4). We did not investigate the remodeling of $C \times 36$ in a rat model of PD.
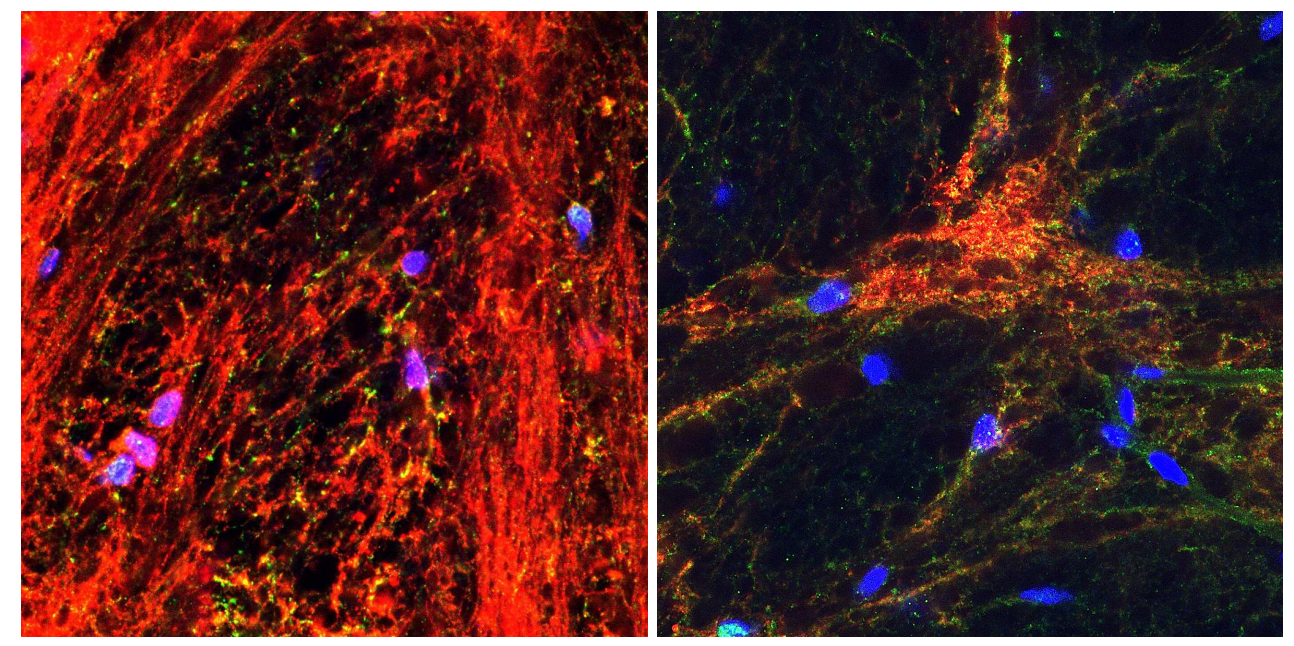

Figure 3.4: Rat GP (left) and EP (right). Clear Cx36 staining (green) is visible on CABAergic neurons (red). The labelling quality is enhanced compared to human tissue.

\subsubsection{Gap junctional coupling controls synchrony in a basic model of the basal ganglia}

Based on our experimental findings of $\mathrm{C} 36$ in the human $\mathrm{C}_{1} \mathrm{Pe}$ and $\mathrm{C}_{1} \mathrm{Pi}$, we used a small network model to demonstrate effect of gap junction coupling in these nuclei. Fig. 3.5 shows the results of our PCA analysis for different gap junction densities, gap junction conductances $g_{G} P_{e}$ and $g_{G P} P_{i}$, and cortical input. We compared the number of PCs as we increased $\mathrm{g}_{G_{P}} P_{e}$ and $\mathrm{g}_{C_{i}} \mathrm{P}_{i}$ in order to model modulation of the gap junction conductance by dopamine. As gap junctions in $\mathrm{C}_{1} \mathrm{Pi}$ cannot have any effects upstream, the variation of PCs in STN and C.Pe dependent on $g_{G_{1} P_{i}}$ indicates solely the level of variation with random input and initial conditions.

For sparse gap junction coupling and uncorrelated cortical input, the increase in gap junction conductance induced moderate synchronization as indicated by a decrease in the number of PCs (Fig. 3.5(a)). Similar results were achieved with cor- 
related cortical input to the STN (Fig. 3.5(b)). In contrast, cortical inputs impacted synchronization when numerous gap junctions were present. When cortical input was uncorrelated, higher gap junction conductances in the sparse architecture led to synchronization, generating a minimum of 4 PCs (Fig. 3.5(c)). Under the influence of correlated cortical input, a minimum of 2 PCs could be achieved, indicating almost complete synchronization (Fig. 3.5(d)). Thus, in our computational model, synchrony in the basal ganglia depended on pallidal gap junction coupling as well as the cortical input to STN. Although the STN itself did not contain any gap junctions, pallidal gap junctions could influence its synchrony. Furthermore, reducing the number of gap junctions to on average 0.25 per cell led to desynchronization at medium gap junction conductances (data not shown).

\subsection{Discussion and conclusions}

We detected $\mathrm{C} \times 36$ in the human putamen, $\mathrm{C}_{1} \mathrm{Pe}$ and $\mathrm{C}_{1} \mathrm{Pi}$, suggesting gap junction coupling in these nuclei. In contrast, no Cx36 was found in the human STN. Cx36 remodeling was seen in the $C_{1} P$ e and $C_{1} P i$ of the PD patients. In a small network model of the basal ganglia, we demonstrated that numerous high-conductance gap junction coupling rendered the GPe more susceptible with cortical inputs transmitted via the STN. Cells of the STN also showed high synchronization, although they were not coupled via gap junctions themselves. We suggest pallidal gap junction coupling as a mechanism for the transmission and reinforcement of neural synchrony in the dopamine depleted basal ganglia, which can be tested in vivo or in vitro in an animal model of PD. We predict that the application of a gap junction blocker directly on the C.Pe and C.Pi will decrease neural synchronization in the dopamine-depleted basal ganglia. Should this prove true, it would be interesting to see how gap junction blocking affects the motor signs of the animal. A direct involvement of gap junction coupling in the pathophysiology of PD would open up novel treatment possibilities, including pharmacological modulation of gap junctions.

Our findings of $\mathrm{C} 36$ in the human C.Pe and $\mathrm{C}_{1} \mathrm{Pi}$ are novel. $\mathrm{C} \times 36$ has however already been detected in the healthy rat GP [Rash et al., 2000]. As with our human tissue, the level of $\mathrm{C} \times 36$ in the rat C.P was low compared to $\mathrm{Cx36}$ in the rat striatum. Kita [1994] also described single gap junctions on PV-negative neurons in the rat GP using electron microscopy. While the messenger ribonucleic acid (mRNA) of Cx36 has been found in rat STN and GP [Vandecasteele et al., 2007], no gap junctions were found the rat STN using electron microscopy [Chang et al., 1983]. We also did not find significant levels of Cx36 in the human STN. Gao et al. [2013] showed 


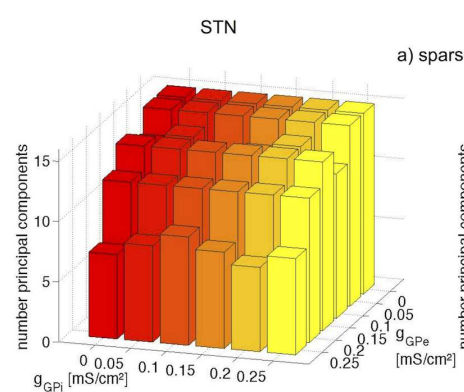

GPe
a) sparse gap junctional coupling; input from Cortex uncorrelated

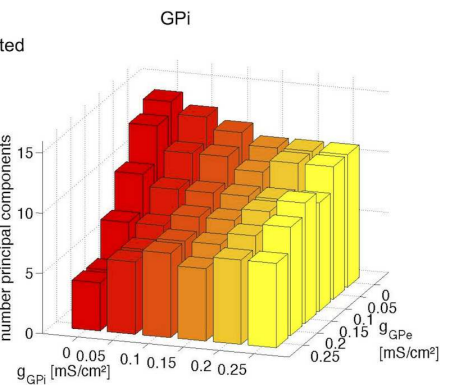

b) sparse gap junctional coupling; input from Cortex correlated
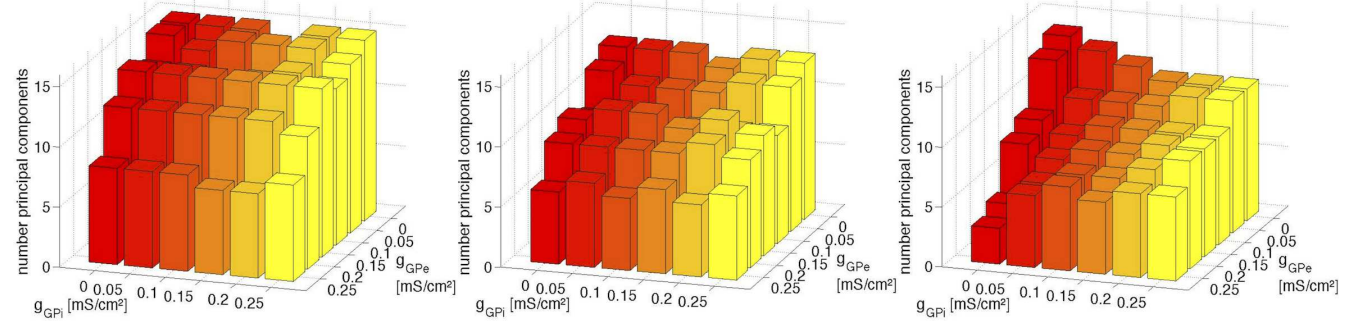

c) numerous gap junctional coupling; input from cortex uncorrelated
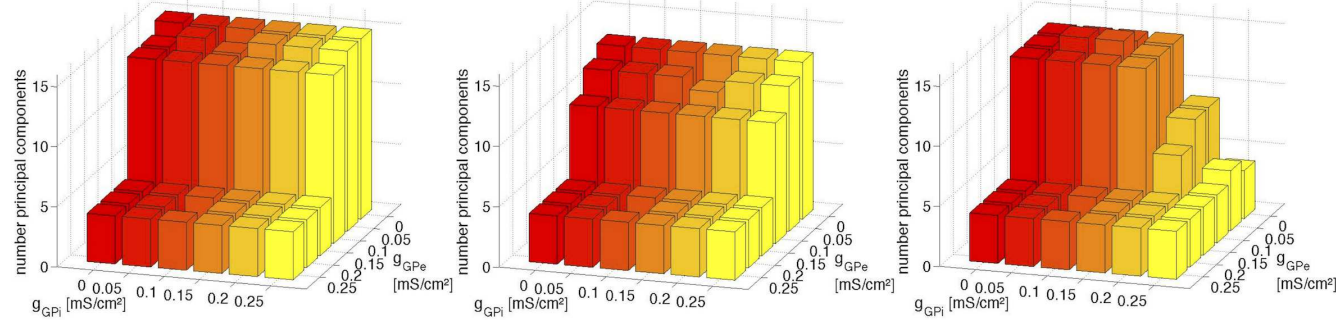

d) numerous gap junctional coupling; input from cortex correlated
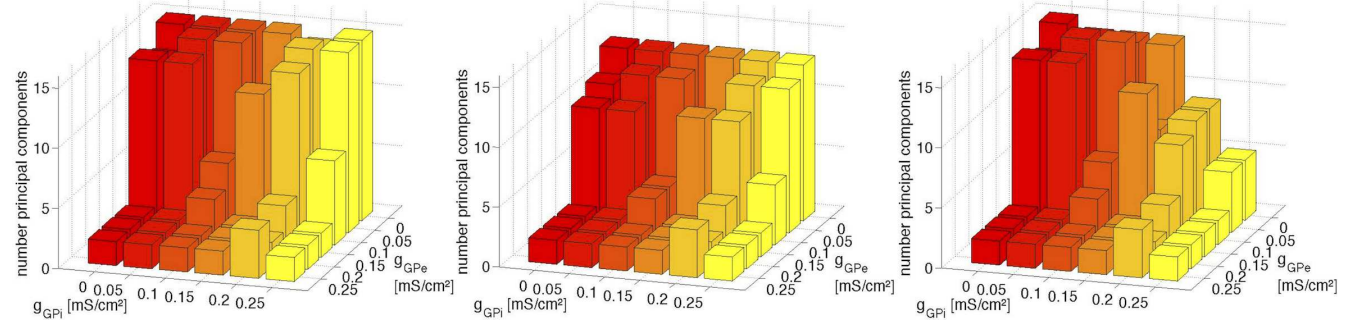

Figure 3.5: Gap junctions affect synchrony in a basic computational model of the basal ganglia. We show the results of the principal component analysis of neural activity in the STN (first column), GPe (second column) and C.Pi (third column) at sparse (a and b) and numerous ( $c$ and $d$ ) gap junction coupling in $\mathrm{C}_{1} \mathrm{Pe}$ and $\mathrm{C}_{1} \mathrm{Pi}$ as well as with uncorrelated ( $a$ and $c$ ) and uncorrelated (b and d) inputs from the cortex. Bars show the number of principal components dependent on gap junction conductance in the $C_{1} P e\left(g_{C_{1}} P_{e}\right)$ and in the $C_{1} P i\left(g_{C_{l}} P_{i}\right)$. Points with gap junction conductance zero indicate the reference without gap junction coupling. Colors are only used for better visibility. 
up-regulated expression of Cx36 in the motor cortex and striatum of a rat model of PD. In the striatum, they found an increase of 38\% using immunohistochemistry and $15 \%$ with immunoblotting. Similar, our immunohistochemistry of the putamen yielded a $50 \%$ increase in the number of $\mathrm{C} \times 36$ spots and a $42 \%$ increase in their area.

The use of human tissue imposed limitations on our study. First, the total number of subjects was low, but all of them showed Cx36 in C.Pe and C.Pi. Furthermore, the tissue had post-mortem delays between three and eight hours. Although this is a short time for processing human tissue, some proteins may have already degenerated. Moreover, the tissue has been fixed in formalin, which restricts the labeling quality and hinders the possibility of immunoblotting. The quality of the human tissue is therefore not comparable to directly fixed animal tissue. Additionally, most PD patients in this study were treated with L-3,4-dihydroxyphenylalanine (L-Dopa), which may also have effects on Cx36 expression. However, PD patients \# 7 and \#11 did not receive L-Dopa the year before their death and their Cx36 levels were not lower than those of the other PD patients. In general, post-mortem studies do not allow a definite statement on whether the observed changes are causal, compensatory or epiphenomenal for pathology. Studies with animal models of PD will be appropriate to address these questions in further detail.

We also considered limitations related to gap junction functionality and their role in the basal ganglia. Firstly, detection of Cx36 on cell membranes does not directly imply gap junction coupling, but can also indicate hemi-channels or non-functional gap junctions. Fukuda et al. [2006] mentioned that 2-5\% of Cx36 spots indicate functional gap junctions in the open configuration, whereas Curti et al. [2012] estimate about $0.1 \%$ of $\mathrm{C} \times 36$ spots to be open, functional gap junctions. In pathological states such as PD, the average function of the gap junctions may change. Nevertheless, the occurrence and the detected levels of $\mathrm{C} \times 36$ give indications of gap junction coupling. We neglected potential gap junction coupling between different nuclei since gap junctions typically occur between adjacent cells [Soehl et al., 2005] of the same type [Katsumaru et al., 1988, Galarreta and Hestrin, 1999, Gibson et al., 1999, Tamás et al., 2000, Connors et al., 2004, Hestrin and Galarreta, 2005]. However, we cannot exclude the possibility of gap junction coupling for example to and from the striatum as gap junction coupling between different nuclei is potentially not explored yet. Secondly, we did not investigate the expression of Cxs other than Cx36. Although gap junction coupling between $C_{1} A B A$ ergic neurons mainly involves $C \times 36$ [Connors et al., 2004, Soehl et al., 2005, Fukuda et al., 2006, Dere, 2012], glutamatergic neurons may express other Cxs such as Cx45 [Ma et al., 2011]. Thus, we cannot provide 
a definite statement on the absence of gap junctions in the human STN.

Thirdly, for gap junction coupling to occur, we need to assume that anatomical contacts exist between cells. While local axon collaterals are extensively present in the human $\mathrm{C}_{1} \mathrm{Pe}$ [Francois et al., 1984], they seem to be low in number in the $\mathrm{C}_{1} \mathrm{Pi}$ [Parent and Parent, 2004]. It is therefore questionable whether Cx36 in the human $\mathrm{C}$ Pi can lead to functional gap junction coupling. Fourthly, we did not consider heterogeneity within the GPe. Recent studies have demonstrated functional as well as structural dichotomy in the GPe [Mallet et al., 2012, Mastro et al., 2014, Dodson et al., 2015, Abdi et al., 2015]. As gap junctions between cortical interneurons typically couple cells of the same type (see above), it is possible that also pallidal gap junctions couple only within one population, or within both populations. Future studies may target more specifically on which kind of C.Pe neurons Cx36 is located. We noticed that $\mathrm{C} \times 36$ was not distributed homogeneously within the $\mathrm{C}_{1} \mathrm{Pe}$, and that some clusters of cells had high levels of $\mathrm{C} \times 36$, whereas the protein was almost absent in other regions. This observation motivated the modeling of gap junctions in small clusters (Fig. 3.1(b)), but remains to be linked to functional connectivity.

Finally, the computational model demonstrated synchronizing roles of pallidal gap junctions depending on their strength and architecture, but may not realistically reproduce the neural dynamics in the whole basal ganglia. Specifically, it cannot replicate oscillations within different frequency ranges. In Chapter 4, we elaborate this model, including a larger number of cells, random synapse exclusions, and quantitative estimates of connectivity from experimental data. In particular, we then average the results of four simulations and present averages as well as the standard error of the mean.

Based on our findings of $C_{x 36}$ in the human $C_{1} P e$ and $C_{1} P i$, we hypothesize the existence of gap junction coupling in these nuclei. Further, we suggest a remodeling of gap junctions after neural injury and an increase of gap junction conductance after dopamine depletion in PD. In PD patients, dopamine loss also occurs in the GPe $(-82 \%)$ and $\mathrm{C}_{1} \mathrm{Pi}(-51 \%)$ [Rajput et al., 2008]. Although the study of Rajput et al. [2008] also detected dopamine in bypassing axons through the $\mathrm{C}_{1} \mathrm{Pe}$, the dopamine level in the $\mathrm{C}_{1} \mathrm{Pe}$ itself can be expected to be of a similar magnitude. Application of dopamine to the G.P of rat models of PD improved the rats' motor behavior [Galvan et al., 2001]. Dye-coupling in the striatum of 6-OHDA rat models of PD, indicative of gap junction coupling, can increase up to four-fold [Cepeda et al., 1989]. A similar increase in gap junction coupling in the $\mathrm{C}_{1} \mathrm{Pe}$ and $\mathrm{C}_{1} \mathrm{Pi}$ after dopamine depletion would be sufficient 
to trigger strong synchronization in the basal ganglia.

In our computational model, small changes in gap junction conductance of about $0.05 \frac{\mathrm{mS}}{\mathrm{cm}^{2}}$ could shift the basal ganglia to a synchronised state. We stress that the pathology of PD may also involve altered gap junction coupling in the cortex, striatum, substantia nigra and retina. Using a computational model, Damodaran et al. [2014] described how altered gap junction coupling between fast-spiking interneurons of the striatum can lead to an imbalance of the direct and indirect pathway in PD. gap junctions between interneurons of the cortex could be involved in the generation of $\theta$ or $\gamma$ oscillations [Traub et al., 2000]. Modulation of the gap junctions between dopaminergic cells of the substantia nigra pars compacta [Vandecasteele et al., 2005] may contribute to cell death [Sung et al., 2007] and it can be speculated that similar processes occur in the retina.

Gap junction remodeling may also depend on factors other than neural injury and the dopamine level. Palacios-Prado et al. [2013] demonstrated an increase in gap junction coupling with decreasing intracellular magnesium concentration. Given that the intracellular magnesium concentration is decreased in the resting brain of patients with PD and other neurological diseases [Barbiroli et al., 1999], gap junction coupling may incleed increase in PD. In contrast, Sung et al. [2007] showed that overexpression of $\alpha$-synuclein, as seen in PD brains, can decrease gap junction coupling. The role of gap junctions in health and disease is far from understood and their interplay with chemical synapses can sculpt neural network dynamics in various ways. 



\title{
Sparse pallidal connectivity shapes synchrony in a network model of the basal ganglia
}

\begin{abstract}
Neural synchrony in the basal ganglia, especially in the beta frequency band (13$30 \mathrm{~Hz}$ ), is a hallmark of Parkinson's disease and considered as antikinetic. In contrast, the healthy basal ganglia show low levels of synchrony. It is currently unknown where synchrony and oscillations arise in the parkinsonian brain and how they are transmitted through the basal ganglia, as well as what makes them dependent on dopamine. The external part of the globus pallidus has recently been identified as a hub nucleus in the basal ganglia, possessing intrinsic inhibitory connections and possibly also gap junctions. In a conductance-based network model of the basal ganglia, the combination of sparse, high-conductance inhibitory synapses and sparse, low-conductance gap junctions in the external part of the globus pallidus could effectively desynchronize the whole network. However, when gap junction coupling became strong enough, the effect was impeded and the network synchronized. In particular, sustained periods of beta coherence occurred between some neuron pairs. As gap junctions can change their conductance with the dopamine level, we suggest pallidal gap junction coupling as a mechanism contributing to the development of beta synchrony in the parkinsonian basal ganglia. ${ }^{1}$
\end{abstract}

\footnotetext{
1 Adapted from Schwab et al. [2016], submitted.
} 


\subsection{Introduction}

After severe dopamine depletion in the basal ganglia of Parkinson's disease (PD) patients, or of animal models, robust synchrony of neural activity in the beta band $(13-30 \mathrm{~Hz})$ emerges [Brown, 2003]. Nevertheless, the origins of such synchrony and low-frequency oscillations, which have been suggested to underlie motor deficiencies, remain debated (for review, see Galvan et al. [2015]). Many studies highlight contributions of motor cortex [Magill et al., 2001, Tachibana et al., 2011, Chu et al., 2015] or the system of the external part of the globus pallidus (C.Pe) and subthalamic nucleus (STN) for the generation of synchrony and oscillations [Plenz and Kital, 1999, Terman et al., 2002, Tachibana et al., 2011]. Recently, also other feedback loops have been acknowledged, for example between C.Pe and striatum [Gittis et al., 2014, Fujiyama et al., 2015]. Most theories for the generation of synchrony and oscillations have in common that the C.Pe is involved, either in a direct feedback loop or in the transmission of activity to downstream nuclei of the basal ganglia. For example, beta oscillations could be generated in cortex and amplified by the STN-G.Pe circuit which is resonating at a similar frequency [Pavlides et al., 2015].

As the C.Pe has a very central position in the basal ganglia, it might be ideally located to orchestrate synchrony in the basal ganglia [Kita, 2007, Schwab et al., 2013a, Gittis et al., 2014]. It is remarkable that the G.Pe has a very rich intrinsic connectivity [Sadek et al., 2007], including few, but effective inhibitory synapses [Sadek et al., 2007, Bugaysen et al., 2013] and possibly also gap junctions [Schwab et al., 2014, Phookan et al., 2015]. Furthermore, particularly prominent activity changes occur in $\mathrm{C}_{1} \mathrm{Pe}$ after dopamine depletion. As under healthy conditions, G.Pe does not show any correlations between the activity of pairs of neurons [Nini et al., 1995, Bar-Gad et al., 2003], an active mechanism of decorrelation may be present in this nucleus [Bar-Gad et al., 2003]. However, after dopamine depletion, C.Pe shows strong correlations in the beta frequency band [Nini et al., 1995]. It has therefore been suggested that the mechanism of active decorrelation fails in PD [Bar-Gad et al., 2003], where dopamine might play an important role. Local field potentials (LFPS) in the healthy basal ganglia show only short beta periods [Feingold et al., 2015], whereas these periods are prolonged or continuous in parkinsonism [Brown, 2003]. Up to now, this mechanism for decorrelation of such beta epochs is unknown.

Besides several cellular processes that change the phasic response of single neurons, decorrelation of G.Pe by synaptic interactions has been proposed [Coldberg et al., 2013, Wilson, 2013, Terman et al., 2013]. C.Pe neurons are subject to lateral inhibition, and although the number of those synapses is small, they seem to be 
highly efficient [Bugaysen et al., 2013]. Since the phase response curve (PRC) of G.Pe neurons includes a small negative part [Coldberg et al., 2013], called a type II PRC, lateral inhibition could decorrelate pallidal activity [van Vreeswijk et al., 1994, Hansel et al., 1995, Goldberg et al., 2013, Terman et al., 2013]. Desynchronization of a neuron pair by lateral C.Pe inhibition has also been described experimentally by Sims et al. [2008]: when coupling two G.Pe neurons via an artificial inhibitory current, the neurons became decorrelated. This might explain the absence of correlations in $\mathrm{C}$ Pe under healthy conditions, although it is not clear if the sparse architecture allows such efficient decorrelation. After dopamine depletion, lateral inhibition in $\mathrm{G}_{\mathrm{Pe}}$ might even be increased [Miguelez et al., 2012], but apparently fails to thoroughly decorrelate the basal ganglia.

Here, we show in a neural network model that sparse inhibitory coupling within $\mathrm{C}_{1} \mathrm{Pe}$ is able to effectively desynchronize pallidal activity, and activity in both STN and the internal part of the globus pallidus $\left(\mathrm{C}_{1} \mathrm{Pi}\right)$ which are targeted by $\mathrm{G}_{1} \mathrm{Pe}$. Sparse gap junction coupling of low conductance could even boost this decorrelation. However, gap junction coupling, if present in GPe, might change in under parkinsonian conditions [Schwab et al., 2014]. Due to dopamine depletion, the conductance of single gap junctions could be increased [Hampson et al., 1992, Li et al., 2013], and after neural injury, an up-regulation of the number of gap junctions might occur [Belousov et al., 2012]. Increased gap junction coupling in C.Pe hindered desynchronization in our model and thus promote the transmission of synchronized oscillations to the basal ganglia. Both gap junction and inhibitory coupling are expected to be very sparse in the globus pallidus [Sadek et al., 2007, Bugaysen et al., 2013, Schwab et al., 2014], and we show in our model that this sparsity does not stand in contrast to the importance of these connections, being able to control synchrony in the basal ganglia.

\subsection{Methods}

\subsubsection{Basal ganglia network model}

Based on the work of Terman et al. [2002], we implemented a conductance-based network model of the basal ganglia in MATLAB [Mathworks, 2012]. It included in total 500 cells (100 STN, 300 G.Pe and 100 G.Pi cells), or 50 cells (10 STN, 30 GPe and $10 \mathrm{CPi}$ cells) in the small version of the model. Neurons were connected via excitatory and inhibitory connections similar to the synaptic architecture of Hahn and Mclntyre [2010], shown in Fig. 4.1. In contrast to the model of Hahn and Mclntyre 
[2010], intrinsic inhibitory synapses of the GPe in our model connected also to more distant neurons. To make synaptic coupling sparse and efficient, as indicated by experiments [Baufreton et al., 2009, Bugaysen et al., 2013], we randomly excluded 50\% or $80 \%$ of the inhibitory GPe-G.Pe synapses and $20 \%$ of the other chemical synapses: from $C_{1} P$ e to STN and to $C_{1} P i$, as well as from STN to $C_{1} P e$ and to $C_{1} P i$.

Finally, we added gap junctions as ohmic resistors between spatially close neurons. Also gap junctions were excluded randomly to $50 \%$ or $80 \%$. STN neurons were driven by cortical excitatory input, $\mathrm{C}_{1} \mathrm{Pe}$ and $\mathrm{C}_{1} \mathrm{Pi}$ neurons by inhibitory input from striatum. Independent, thus uncorrelated white noise was added to all neuron inputs. Chemical synapses were described by

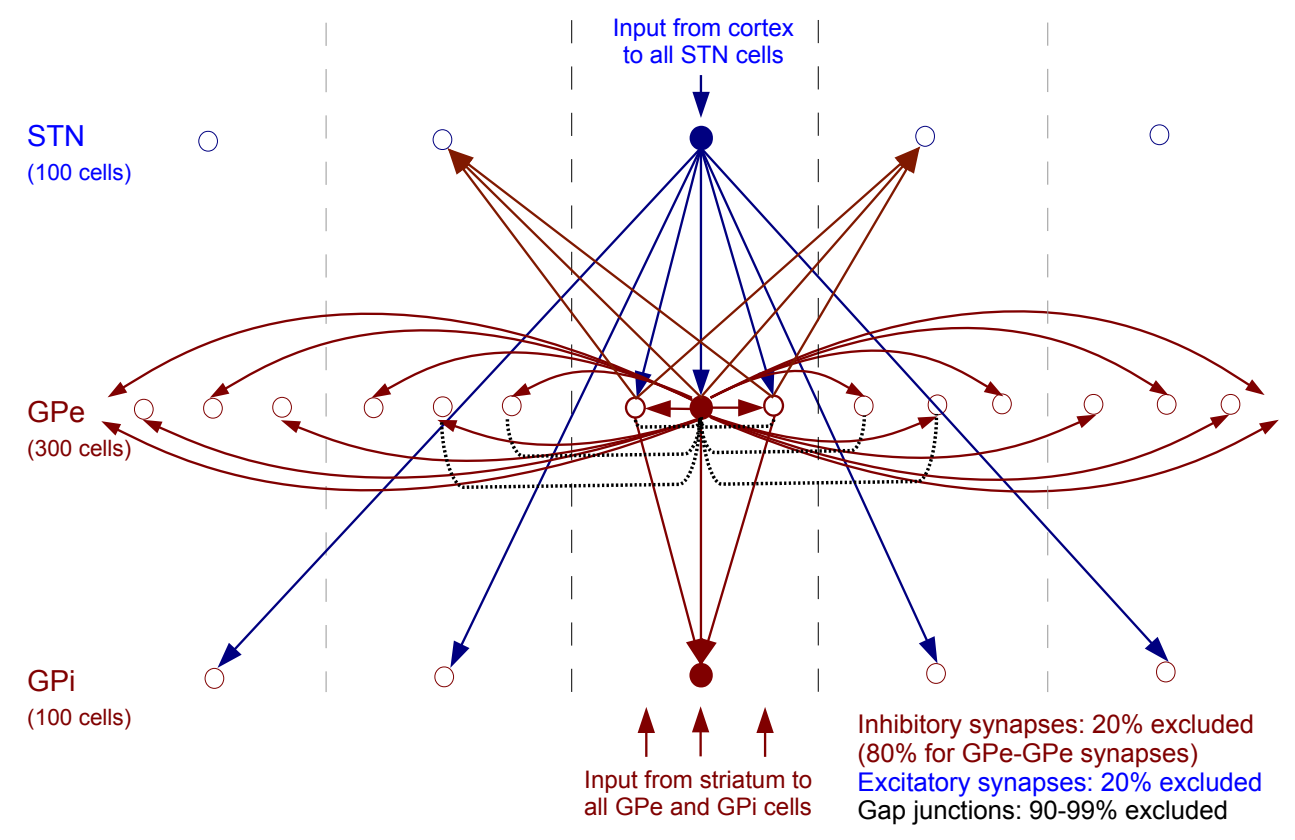

Figure 4.1: Synaptic architecture of the basal ganglia network model, based on Hahn and Mclntyre [2010], before synapses were randomly excluded. Neurons target other neurons in their column more than distant ones, accounting for segregated pathways in the basal ganglia. Gap junctions are placed between spatially close C.Pe neurons. We randomly excluded both chemical synapses and gap junctions shown in this figure, leading to a sparse connectivity.

$$
I_{s y n}(V)=g_{s y n} s\left(V-E_{s y n}\right)
$$


were $g_{s y n}$ is the maximum synapse conductance, $V$ the transmembrane voltage of the presynaptic neuron, and $E_{\text {syn }}$ the synaptic reversal potential. In the following, the index syn will be replaced by nuclei indices, for example by $S T N \rightarrow G_{P} P$. The variable $s$ represents the synaptic open probability and obeyed the following differential equation [Terman et al., 2002]

$$
\begin{aligned}
\frac{d s}{d t} & =\alpha \frac{1}{1+\chi(V)}(1-s)-\beta s, \\
\chi(V) & =e^{-\frac{V-\theta_{g}}{\sigma_{g}}} .
\end{aligned}
$$

$\alpha, \beta, \theta_{g}$, and $\sigma_{g}$ are synaptic constants. Parameter values were taken from Terman et al. [2002] and are summarized in Table 4.1 together with the synaptic strengths $g_{s y n}$ and the reversal potentials $E_{s y n}$. Gap junctions were simulated as ohmic resistors carrying the bidirectional current

$$
I_{G J}(\Delta V)=g_{s y n}\left(V_{1}-V_{2}\right)
$$

with the gap junction conductance $g_{G_{1}}$ and the transmembrane voltages $V_{1}$ and $V_{2}$

\begin{tabular}{|c|c|c|c|c|c|}
\hline & $\theta_{g}$ & $\sigma_{g}$ & $\alpha$ & $\beta$ & \\
\hline STN & $-9 \mathrm{mV}$ & 8 & $5 \mathrm{kHz}$ & $1 \mathrm{kHz}$ & \\
\hline \multirow[t]{5}{*}{$\mathrm{G}_{1} \mathrm{PeE}_{\mathrm{I}} \mathrm{CPi}_{\mathrm{P}}$} & $-37 \mathrm{mV}$ & 2 & $2 \mathrm{kHz}$ & $0.04 \mathrm{kHz}$ & \\
\hline & $g_{G P e \rightarrow S T N}$ & $g_{C i P e \rightarrow G P i}$ & $E_{G P e \rightarrow S T N}$ & $E_{G P e \rightarrow G P i}$ & $E_{G P e \rightarrow G P e}$ \\
\hline & $1.2 \frac{\mathrm{mS}}{\mathrm{cm}^{2}}$ & $1.8 \frac{\mathrm{mS}}{\mathrm{cm}^{2}}$ & $-100 \mathrm{mV}$ & $-100 \mathrm{mV}$ & $-80 \mathrm{mV}$ \\
\hline & $g_{S T N \rightarrow G P i}$ & $g_{S T N \rightarrow G P e}$ & $E_{S T N \rightarrow G P i}$ & $E_{S T N \rightarrow G P e}$ & \\
\hline & $0.4 \frac{\mathrm{mS}}{\mathrm{cm}^{2}}$ & $0.4 \frac{\mathrm{mS}}{\mathrm{cm}^{2}}$ & $0 \mathrm{mV}$ & $0 \mathrm{mV}$ & \\
\hline
\end{tabular}
of the two coupled neurons.

Table 4.1: Synaptic parameters

\subsubsection{Neuron models}

Terman et al. [2002], and later Rubin and Terman [2004], designed conductance-based neuron models to describe C.Pe, STN and GPi activity. We used their model for STN and $\mathrm{CPi}_{\mathrm{P}}$ cells, and adapted their GPe neuron model as described in the following 
subsections. The PRCs of both models were computed with MATCONT [Dhooge et al., 2003] and are shown in Fig. 4.2. Mathematically, MATCONT uses the adjoint method for PRC computation. For numerical details, see Govaerts and Sautois [2006].

\subsubsection{Rubin Er Terman neurons}

Terman et al. [2002] and Rubin and Terman [2004] developed a single-compartment C.Pe neuron model based on voltage-clamp and current-clamp data. The transmembrane potential $V$ of a neuron is described by

$$
\begin{aligned}
\frac{d V}{d t} & =\frac{1}{C_{m}}\left(-I_{L}(V)-I_{K}(V)-I_{N a}(V)-I_{T}(V)-I_{C a}(V)-I_{A H P}(V)\right. \\
& \left.-I_{\text {syn }}(V)-I_{C_{I} J}(\Delta V)+I_{a p p}\right) .
\end{aligned}
$$

$I_{L / K / \mathrm{Na} / T / \mathrm{Ca} / A H P}$ are ionic currents that were governed by the equations

$$
\begin{aligned}
I_{L}(V) & =g_{L}\left(V-E_{L}\right) \\
I_{K}(V) & =g_{K} n^{4}\left(V-E_{K}\right) \\
I_{N a}(V) & =g_{N a}\left(m_{\infty}(V)\right)^{3} h\left(V-E_{N a}\right) \\
I_{I T}(V) & =g_{T}\left(a_{\infty}(V)\right)^{3} r\left(V-E_{C a}\right) \\
I_{C a}(V) & =g_{C a}\left(s_{\infty}(V)\right)^{2}\left(V-E_{C a}\right) \\
I_{A H P}(V) & =g_{A H P}\left(V-E_{K}\right) \frac{[C a]}{[C a]+k_{1}} .
\end{aligned}
$$

The gating variables $X \in\{n, r, h\}$ are functions of both time and voltage, and satisfied the equations

$$
\begin{aligned}
\frac{d X}{d t} & =\phi_{X} \frac{X_{\infty}(V)-X}{\tau_{X}(V)} \\
\tau_{X}(V) & =\tau_{X}^{0}+\frac{\tau_{X}^{1}}{\left[1+\exp \left[-\frac{V-\theta_{X}^{t}}{\sigma_{X}^{t}}\right]\right]} \\
X_{\infty}(V) & =\frac{1}{1+e^{-\left(V-\theta_{X}\right) / \sigma_{X}}} .
\end{aligned}
$$

All rapidly activating channels $X \in\{m, a, s\}$ were treated as instantaneous and are their steady state voltage dependence could directly be computed with $X_{\infty}$ above. Calcium dynamics were governed by the equation

$$
\frac{d[C a]}{d t}=\epsilon\left(-I_{C a}-I_{T}-k_{C a}[C a]\right)
$$


where $\epsilon$ is a buffer constant, $I_{C a}$ a high-threshold calcium current, $I_{T}$ a low-threshold T-type calcium current and $[\mathrm{Ca}]$ the intracellular concentration of calcium ions. We enhanced the calcium pump constant $k_{C_{a}}$ of $C_{1} P e$ and $C_{1} P i$ neurons from 15 to 38, leading to the type II PRC shown in Fig. 4.2(a). The neuron was then spiking at $42 \mathrm{~Hz}$. This spontaneous firing rate has been found in rat pallidal neurons [Deister et al., 2013]. All other parameters were taken from Terman et al. [2002].

\subsubsection{Fujita et al. neurons}

Based on the complex multi-compartment model of Günay et al. [2008], Fujita et al. [2012] described a single-compartment model capturing the soma dynamics of the Günay model. It is also described by a Hodgkin-Huxley formalism:

$$
\begin{aligned}
\frac{d V}{d t} & =\frac{1}{C_{m}}\left(-I_{L}(V)-I_{N a F}(V)-I_{N a P}(V)-I_{K v 2}(V)-I_{K v 3}(V)\right. \\
& -I_{K v 4 f}(V)-I_{K v 4 s}(V)-I_{K C N Q}(V)-I_{C a H}(V)-I_{H C N}(V) \\
& \left.-I_{S K}(V)-I_{S y n}(V)-I_{C J}(\Delta V)\right)+I_{a p p}
\end{aligned}
$$

with the ionic currents

$$
\begin{aligned}
I_{L}(V) & =g_{L}\left(V-E_{L}\right) \\
I_{N a F}(V) & =g_{N a F}\left(m_{N a F}\right)^{3} h_{N a F} S_{N a F}\left(V-E_{N a}\right) \\
I_{N a P}(V) & =g_{N a P}\left(m_{N a P}\right)^{3} h_{N a P S N a P}\left(V-E_{N a}\right) \\
I_{K v 2}(V) & =g_{K v 2}\left(m_{K v 2}\right)^{4} h_{K v 2}\left(V-E_{K}\right) \\
I_{K v 3}(V) & =g_{K v 3}\left(m_{K v 3}\right)^{4} h_{K v 3}\left(V-E_{K}\right) \\
I_{K v 4 f}(V) & =g_{K v 4 f}\left(m_{K v 4 f}\right)^{4} h_{K v 4 f}\left(V-E_{K}\right) \\
I_{K v 4 s}(V) & =g_{K v 4 s}\left(m_{K v 4 s}\right)^{4} h_{K v 4 s}\left(V-E_{K}\right) \\
I_{K C N Q}(V) & =g_{K C N Q}\left(m_{K C N Q}\right)^{4}\left(V-E_{K}\right) \\
I_{C a H}(V) & =g_{C a H} m_{C a H}\left(V-E_{C a}\right) \\
I_{H C N}(V) & =g_{H C N} m_{H C N}\left(V-E_{C a t}\right) \\
I_{S K}(V) & =g_{S K} m_{S K}\left(V-E_{K}\right),
\end{aligned}
$$

where $g_{x}$ are conductances, $E_{x}$ are reversal potentials, and $m, h$, and $s$ are (in)activation state variables. Those state variables followed the differential equation

$$
\frac{d X}{d t}=\frac{X_{\infty}(V)-X}{\tau_{X}(V)}
$$


with $X \in\left\{r, m_{S K}, m_{H C N}, m_{C a H}, m_{K C N Q}, m_{K v 4 s}, h_{K v 4 s}, m_{K v 4 f}, h_{K v 4 f}, m_{K v 3}, h_{K v 3}\right.$, $\left.m_{K v 2}, h_{K v 2}, m_{\mathrm{Na} a}, h_{\mathrm{Na} a}, s_{\mathrm{Na} a}, m_{\mathrm{NaF}}, h_{\mathrm{NaF}}, s_{\mathrm{NaF}}\right\}$, and

$$
\begin{aligned}
& X_{\infty}(V)=X_{\min }+\frac{1-X_{\min }}{1+e^{\left(\theta_{X}-V\right) / k_{X}}} \\
& \tau_{X}(V)=\tau_{X}^{0}+\frac{\tau_{X}^{1}-\tau_{X}^{0}}{e^{\left(\phi_{X}-V\right) / \sigma_{X}^{0}}+e^{\left(\phi_{X}-V\right) / \sigma_{X}^{1}}}
\end{aligned}
$$

for the gating variables. However, for the $I_{\mathrm{NaP}}$ current, the time constant of $s$ was determined by

$$
\tau_{s}=\frac{1}{\alpha_{s}(V)+\beta_{s}(V)}
$$

with

$$
\begin{aligned}
& \alpha_{s}(V)=\frac{A_{s}^{\alpha} V+B_{s}^{\alpha}}{1-e^{\left(V+\frac{B_{\alpha}^{\alpha}}{A_{s}}\right) / K_{s}^{\alpha}}}, \\
& \beta_{s}(V)=\frac{A_{s}^{\beta} V+B_{s}^{\beta}}{1-e^{\left(V+\frac{B_{s}^{\beta}}{A_{s}^{\beta}}\right) / K_{s}^{\beta}}} .
\end{aligned}
$$

The intra-cellular calcium concentration [Ca] obeyed the equation

$$
\frac{d[C a]}{d t}=-\frac{\gamma}{2 F} I_{C a H}-K_{[C a]}\left([C a]-[C a]_{0}\right)
$$

where $F$ is the Faraday constant, $[\mathrm{Ca}]_{0}$ the baseline calcium concentration, $K_{[\mathrm{Ca}}$ the calcium removal rate, and $\gamma$ the cell's surface to volume ratio.

Finally, the $m$ kinetics of $I_{S K}$ followed the Hill equation

$$
m_{\infty}([C a])=\frac{[C a]^{H_{c o e f f}}}{\left(C_{50}\right)^{H_{c o e f f}}+[C a]^{H_{\text {coeff }}}} .
$$

$H_{\text {coeff }}$ is the Hill coefficient and $C_{50}$ the semi-saturation. The time constant for those kinetics reads

$$
\tau_{m}([C a])=\tau^{1}-\frac{\left(\tau^{1}-\tau^{0}\right)[C a]}{[C a]_{\max }}
$$

if $[C a]<[C a]_{\max }$. All parameters were taken from Fujita et al. [2012]. We injected a permanent current $I_{\text {app }}$ of $0.65 \frac{\mu \mathrm{A}}{\mathrm{cm}^{2}}$ to make it spike at $42 \mathrm{~Hz}$. Fig. 4.2 (b) shows its type II PRC. 
(a)

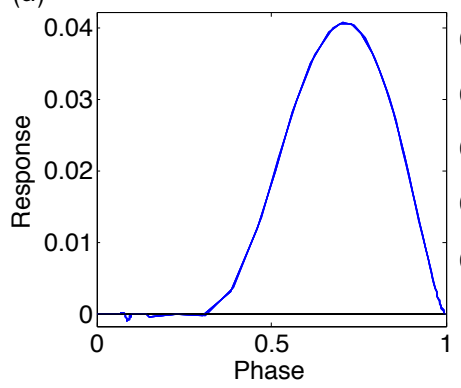

(b)

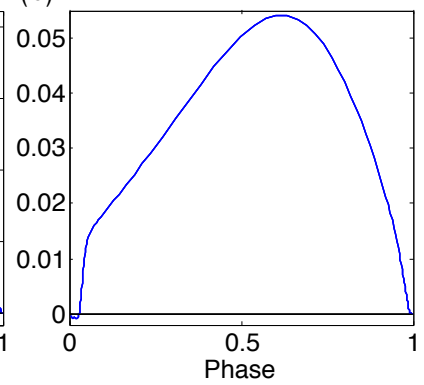

Figure 4.2: PRC of G.Pe model neurons, calculated with MATCONT: (a) Rubin $\varepsilon$ Terman model with enhanced calcium pump, (b) Fujita et al. model. The PRCs of both models show a small negative part for small phases, and a large peak for phases between 0.5 and 0.8 .

\subsubsection{Quantification of synchrony}

To quantify the level of synchrony in a population by a single value, we conducted a principal component analysis (PCA). Spikes were convolved with a Gaussian of standard deviation $7.5 \mathrm{~ms}$. As described in Lourens et al. [2015], we calculated the number of principal components that were needed to describe $95 \%$ of spike time information. A low number of principal components therefore indicates high synchrony. As a control, we also calculated cross-correlation functions (CCFs) of two transmembrane voltage traces, and averaged the obtained CCFs over all pairs in the network. Furthermore, we calculated the magnitude squared coherence between the convolved spike trains of each possible neuron pair. The coherences were either averaged over the whole time course of one simulation, and averaged over all pairs of neurons in one nucleus, or averaged only over sliding 409.6 ms windows in $51.2 \mathrm{~ms}$ steps (coherograms). The term "relative coherence" refers to the summed coherence in one frequency band, for example the beta band, divided by the total coherence summed over all frequencies.

As an indication for the significance of changes in synchrony, we compared the following groups with a two-sample t-test: (1) To test for desynchronization of activity by inhibition, we compared the average number of principal components when the chemical synapse strength was $0.5 \frac{\mathrm{mS}}{\mathrm{cm}^{2}}$ and when it was $1.5 \frac{\mathrm{mS}}{\mathrm{cm}^{2}}$. This was done for both low $\left(0.1 \frac{\mathrm{mS}}{\mathrm{cm}}\right)$ and high gap junction strength $\left(0.5 \frac{\mathrm{mS}}{\mathrm{cm}^{2}}\right)$. (2) To test for desynchronization of activity by low gap junction coupling, we compared the average number of principal components when gap junction coupling strength was zero, and when it was $0.1 \frac{\mathrm{mS}}{\mathrm{cm}^{2}}$, given a medium strength of chemical synapses $\left(1.5 \frac{\mathrm{mS}}{\mathrm{cm}^{2}}\right)$. (3) To test for 
synchronization of activity by high gap junction coupling, we compared the average number of principal components when gap junction coupling strength was $0.1 \frac{\mathrm{mS}}{\mathrm{cm}^{2}}$, and when it was $0.5 \frac{\mathrm{mS}}{\mathrm{cm}^{2}}$, again at medium strength of chemical synapses $\left(1.5 \frac{\mathrm{mS}}{\mathrm{cm}^{2}}\right)$. (4) To test for an increase in beta coherence with high gap junction coupling, we compared the mean beta coherence, averaged over all strengths of chemical synapses, when gap junction coupling strength was zero, and when it was $0.5 \frac{\mathrm{mS}}{\mathrm{cm}^{2}}$. (5) The same as in (4) was done for relative beta coherence.

\subsection{Results}

\subsubsection{Estimated connectivity within the external part of the globus pallidus}

Coupling within the basal ganglia has often been described as sparse [Morris et al., 2003, Baufreton et al., 2009, Bugaysen et al., 2013]. In particular, cells within G.Pe seem to exhibit inhibitory connections only with a probability of around 1\% (Sadek et al. [2007], based on anatomical tracing) or 1.4\% (Bugaysen et al. [2013], based on voltage-clamp). Given a network of $300 \mathrm{C}$ Pe neurons, a connection probability of $1.4 \%$ would be equivalent to on average 2.1 unidirectional synapses per neuron. However, for realistic numbers of neurons - about 45,960 neurons in the rat G.P [Oorschot, 1996] or about 904,365 neurons in the human C.Pe [Wegiel et al., 2014] - we would estimate a much higher number of synapses per neuron. It thus makes more sense to consider the number of inputs that a GPe neuron receives from other neurons. Based on electron microscopy, Sadek et al. [2005] estimated that the number of inputs is between 23 and 194. Here, we investigated even sparser coupling, starting at 3.6 synapses per neuron, corresponding to $80 \%$ synapse exclusion in Fig. 4.1. This value is in the order of the estimated connectivity with $1.4 \%$ connection probability in a 300 cell network ( 2.1 inputs per neuron, see above). 50\% synapse exclusion was equivalent to 9 synapses per neuron.

Also gap junctions, if present in G.Pe, are sparse [Schwab et al., 2014]. Fukuda et al. [2006] mention that about 2-5\% of Cx36 spots in the visual cortex indicate functional gap junctions. This is in rough accordance with the estimations of Curti et al. [2012] that only about $0.1 \%$ of gap junction channels are functional and in the open configuration, under the assumption that each Cx36 spot includes tens to hundreds of gap junction channels [Bennett and Zukin, 2004, Bukauskas et al., 2000]. Supposing that this ratio is similar for G.Pe, i.e. about $1 \%$ of all Cx36 spots indicate functional gap junctions, we rudely approximate the number of functional gap junc- 
tions per neuron based on our human tissue [Schwab et al., 2014]. Healthy tissue had a density of around $900 \mathrm{C} \times 36$ puncta $/ \mathrm{mm}^{2}$. Civen a resolution of the confocal microscope of around $1 \mu \mathrm{m}$ in axial direction, this density scales to $9 \cdot 10^{5}$ puncta/ $/ \mathrm{mm}^{3}$. With a neuron density of 2,485 neurons $/ \mathrm{mm}^{3}$ in the human G.Pe [Wegiel et al., 2014], we get 362 puncta/neuron, and thus on average around 3.6 functional gap junctions per neuron. In PD patients, we detected around 1,300 puncta/ $\mathrm{mm}^{2}$, which relates to roughly 5.2 functional gap junctions per neuron. Again, we modeled even lower densities of gap junctions from 0.6 gap junction per neuron ( $80 \%$ exclusion) to 1.5 gap junctions per neuron (50\% synapse exclusion). We considered here the relation of the total number of gap junctions to the total number of neurons, which should not be confused with the number of gap junction connections that a single neuron makes on average - the latter is a factor two higher.

\subsubsection{Lateral inhibition in Ge desynchronized the basal ganglia, whereas strong gap junction coupling synchronized}

We simulated the model, including 500 Rubin $\varepsilon$ Terman neurons, as described in Section 4.2 for 18 seconds. For the analysis, we neglected the first $2 \mathrm{~s}$ of each simulation. Fig. 4.3 shows how synchrony, quantified by a PCA, was modulated by lateral inhibition in G.Pe and by gap junctions, both excluded to $50 \%$. All results are shown as the mean $+/$ - standard error of the mean (SEM), calculated after four simulation trials with different random synapse exclusion and initial conditions. Independent of the gap junction strength, inhibition led to a relative desynchronization of the whole network, including STN and C.Pi, seen as an increased number of principal components when the strength of inhibition increased $(p<0.05$ for all nuclei, see Section 4.2.3). Slight gap junction coupling on top of inhibition was able to further desynchronize G.Pe $(p<0.01)$. However, when gap junctions became strong enough $\left(0.3 \frac{\mathrm{mS}}{\mathrm{cm}^{2}}\right.$ or larger), a synchronization was visible in all three nuclei $(p<0.01)$. When even more gap junctions and synapses were excluded, for example $80 \%$ as shown in Fig. 4.4, all three described effects were still visible $(p<0.05)$ but decreased in magnitude. Further exclusion of more than $80 \%$ of synapses failed to show similar effects (not shown).

The PCA is not an appropriate method to detect out-of-phase synchrony. We therefore confirmed our PCA results with CCFs, averaged over all pairs of the network (not shown). CCFs were either flat, corresponding to a high number of principal components, or showed a peak at time lag zero, decreasing in height with stronger inhibition. Low gap junction coupling slightly decreased the CCF peaks, but increased 
them for higher strengths. In both cases, SEMs were small compared to the mean number of principal components, indicating that the concrete connection architecture played only a minor role for synchrony.

(a)

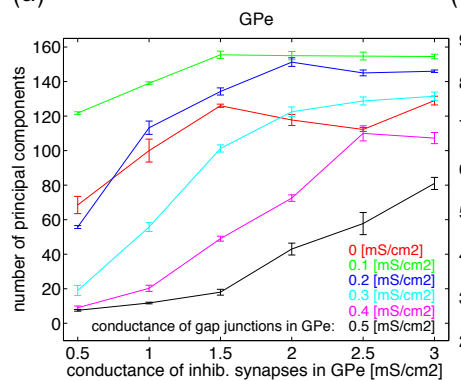

(d)

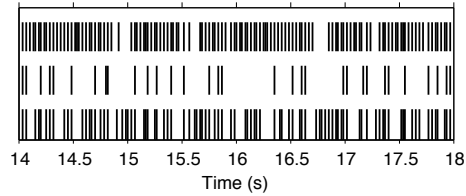

(b)

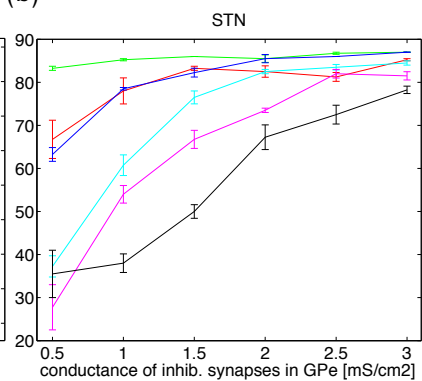

(e)

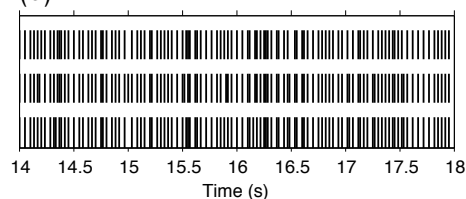

Figure 4.3: PCA of neural activity in C.Pe (a), STN (b) and CPi (c) when 50\% of all gap junctions and $50 \%$ of all lateral C.Pe synapses were randomly excluded. Bars indicate the SEM. Inhibition within C.Pe was able to effectively desynchronize the whole network, including STN and $\mathrm{C}_{1} \mathrm{Pi}$. While low gap junction coupling in $\mathrm{CPe}$ further desynronized GPe, medium to high values of gap junction strength could synchronize activity. (d) Example raster plot of spikes in three example GPe neurons when both gap junction coupling and synchrony was low. Gap junction conductance: $0.1 \frac{\mathrm{mS}}{\mathrm{cm}^{2}}$, chemical synapse conductance: $1.5 \frac{\mathrm{mS}}{\mathrm{cm}^{2}}$. (e) Example raster plot for synchronized spiking in C.Pe when gap junction coupling was high. Firing rates increased and bursts often occurred at the same time in all three spike trains. Crap junction conductance: $0.5 \frac{\mathrm{mS}}{\mathrm{cm}^{2}}$, chemical synapse conductance: $1.5 \frac{\mathrm{mS}}{\mathrm{cm}^{2}}$.

\subsubsection{High gap junction coupling increased beta coherence}

Synchrony in the parkinsonian basal ganglia is typically found in the beta band [Nini et al., 1995, Brown, 2003]. In our model, the emergence of beta synchrony, here restricted to the core of the beta band $(20-25 \mathrm{~Hz})$, depended on gap junction coupling, if the coupling was not very sparse (Fig. 4.5 and 4.6). For 50\% synapse and gap junction exclusion (Fig. 4.5), beta coherence was suppressed for low gap 
(a)

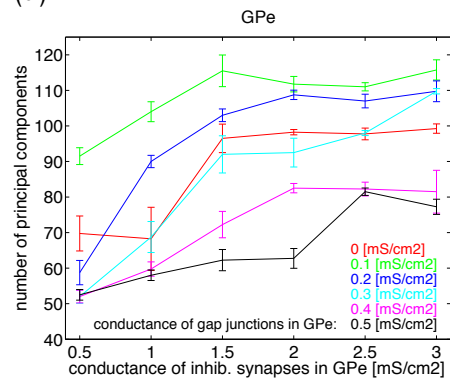

(d)

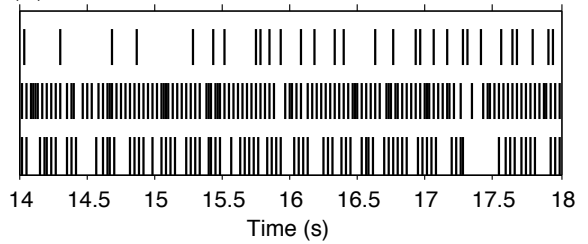

(b)

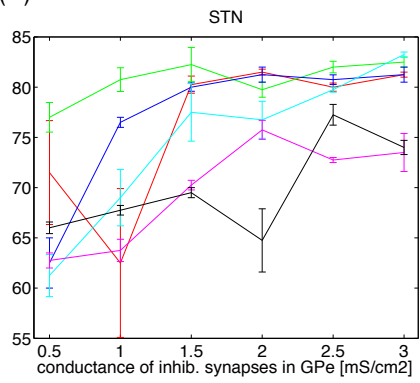

(c)

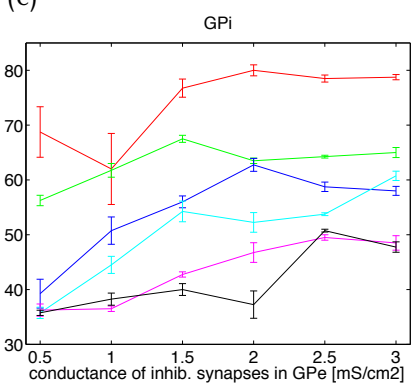

(e)

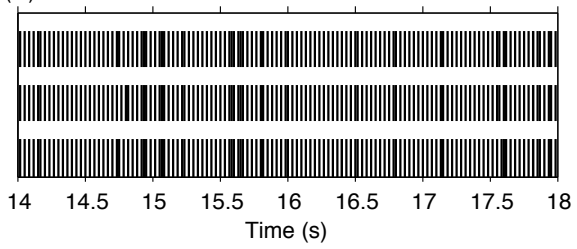

Figure 4.4: PCA of neural activity in GPe (a), STN (b) and GPi (c) when $80 \%$ of all gap junctions and $80 \%$ of all lateral C.Pe synapses were randomly excluded. Bars indicate the SEM. In comparison to Fig. 4.3, desynchronization by inhibition, but also synchronization by gap junctions is weaker. Still, both effects are clearly visible. $(\mathrm{d}, \mathrm{e})$ Example raster plots for low and high gap junction coupling, respectively. Gap junction conductance: $0.1 \frac{\mathrm{mS}}{\mathrm{cm}^{2}}$ (d) and $0.2 \frac{\mathrm{mS}}{\mathrm{cm}^{2}}$ (e), chemical synapse conductance: 1.5 $\frac{m S}{c m^{2}}$.

junction conductances, and emerged for high conductances. Coherence, averaged over all values for different chemical synapse strengths, significantly increased from zero gap junction coupling to high gap junction coupling $\left(0.5 \frac{\mathrm{mS}}{\mathrm{cm}^{2}}\right)(p<0.01$, see Section 4.2.3). Also relative beta coherence, i. e. the fraction of beta coherence relative to coherence in general, showed a similar trend $(p<0.01)$, although the magnitude of those changes was only small. When looking at example coherograms (Fig. 4.5(a-d)), we can see that in some neuron pairs, long epochs of beta coherence emerged for high gap junction coupling. When both gap junctions and inhibitory synapses were very sparse (Fig. 4.6), any effects on beta coherence were not significant any more. We stress that synchrony did not occur only in the beta band: for example, some pairs showed coherence in a wide frequency band, as in the middle row of Fig. 4.5(c). 
(a)

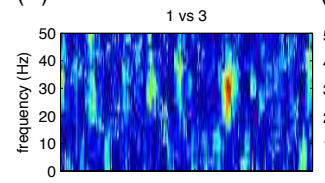

(b)
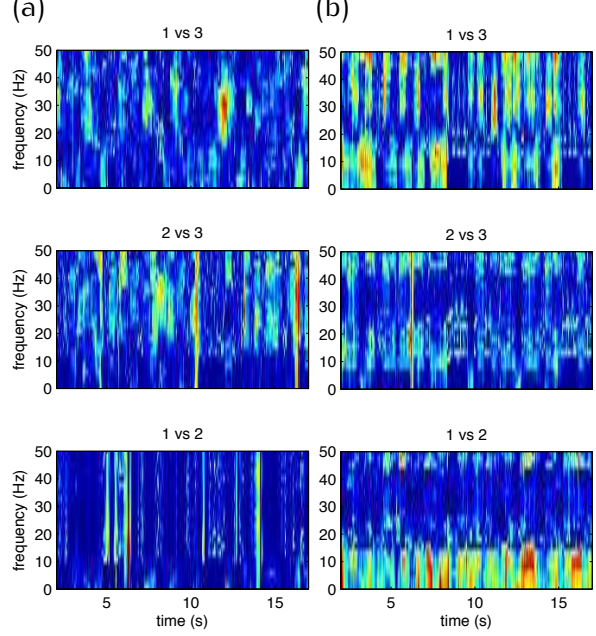

(e)

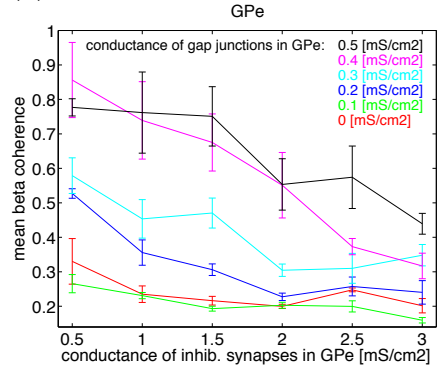

(f)
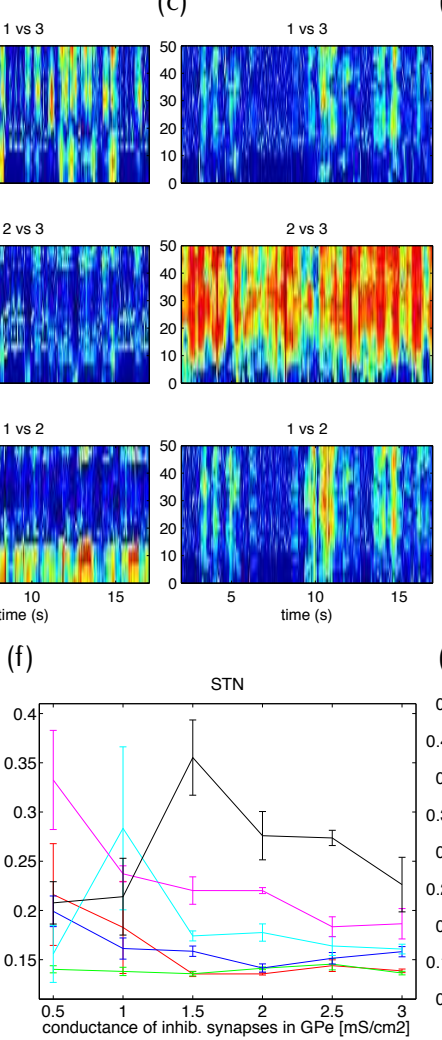

(d)
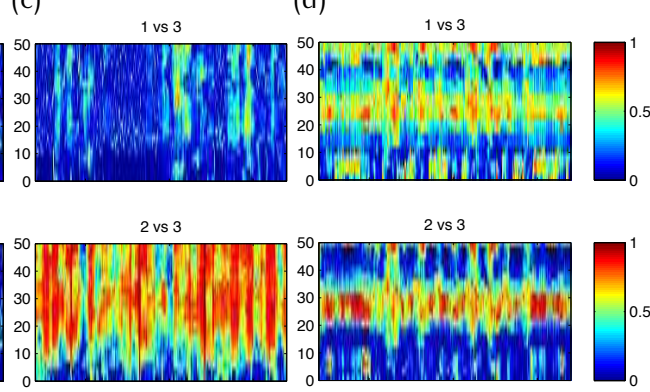

1 vs 2
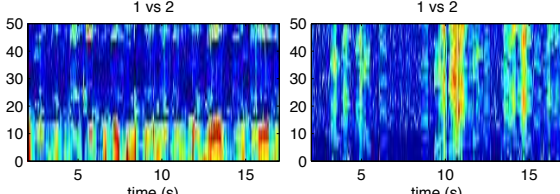

time (s)

(g)
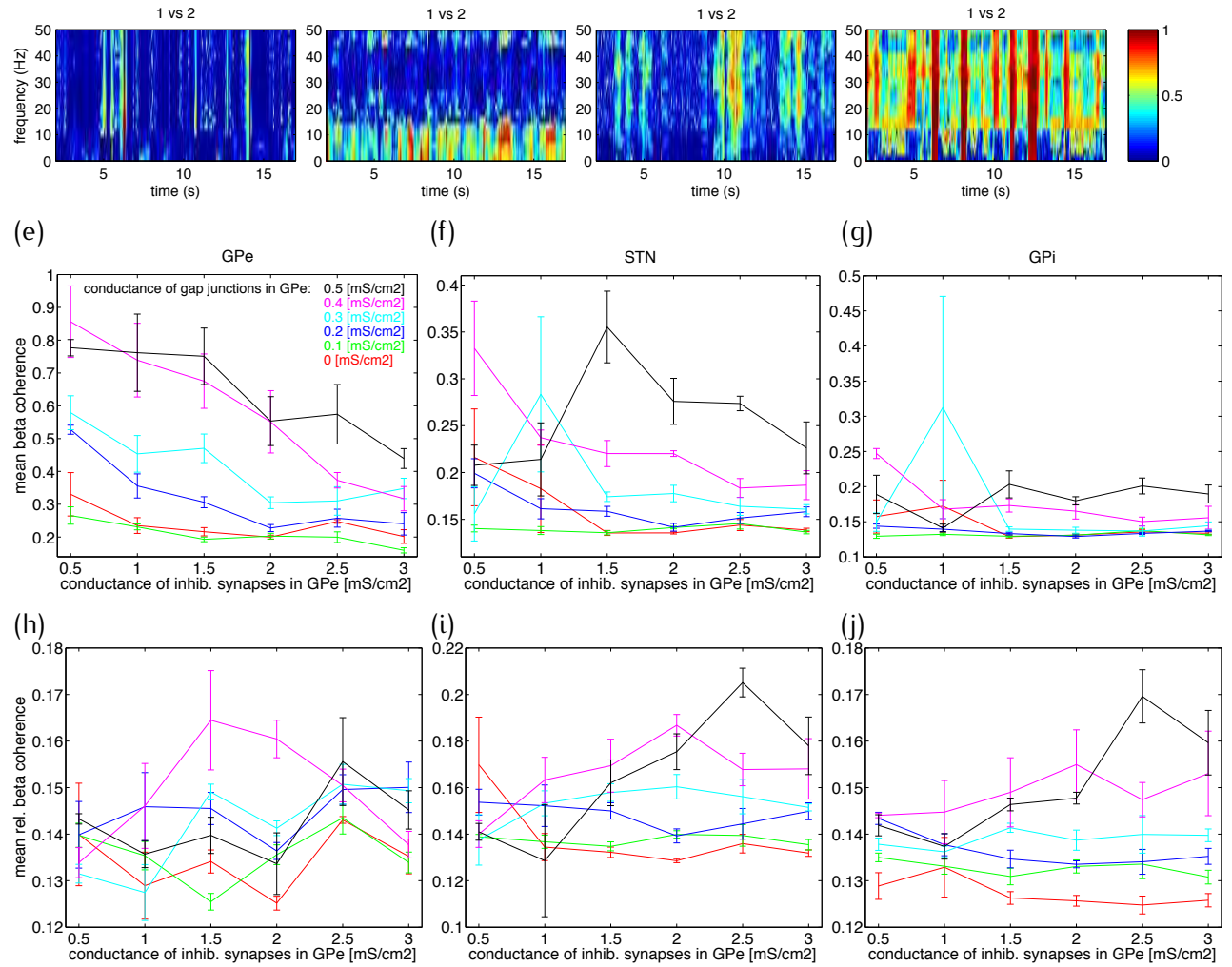

(i)

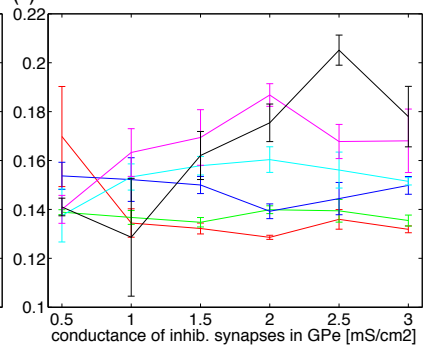

(j)

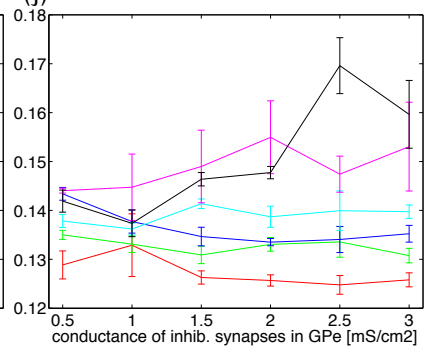

Figure 4.5: Coherograms for 50\% synapse and gap junction exclusion: examples of coherence between cell pairs evolving over time (a) without, (b) with low $\left(0.1 \frac{\mathrm{mS}}{\mathrm{cm}^{2}}\right)$, (c) medium $\left(0.3 \frac{\mathrm{mS}}{\mathrm{cm}^{2}}\right)$ and $(\mathrm{d})$ high $\left(0.5 \frac{\mathrm{mS}}{\mathrm{cm}^{2}}\right)$ gap junction coupling. Beta epochs become less frequent with low gap junction coupling, but become dominant for high gap junction coupling. (e-g) Average beta coherence in G.Pe (e), STN (f) and C.Pi (g), decreases with slight gap junction coupling and increases with high gap junction coupling. (h-j) Relative beta coherence (normalized by the total coherence) in (h) $\mathrm{C}_{1} \mathrm{Pe}$, (i) STN and (j) $\mathrm{C}_{1} \mathrm{Pi}$ shows a similar trend as the absolute beta coherence. 
(a)
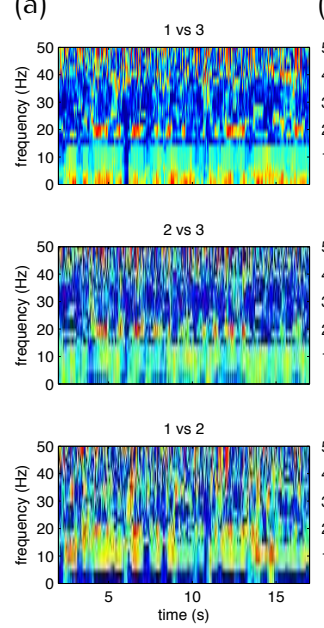

(e)

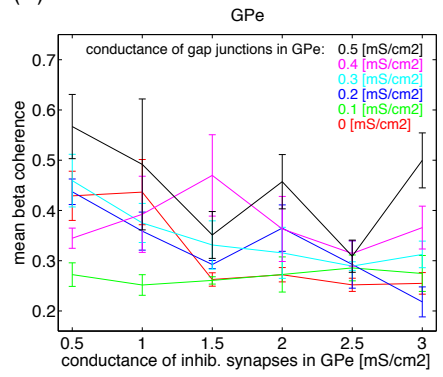

(h)

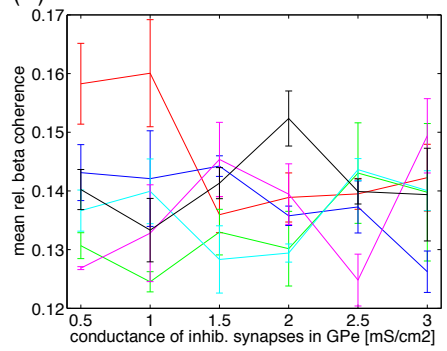

(c)
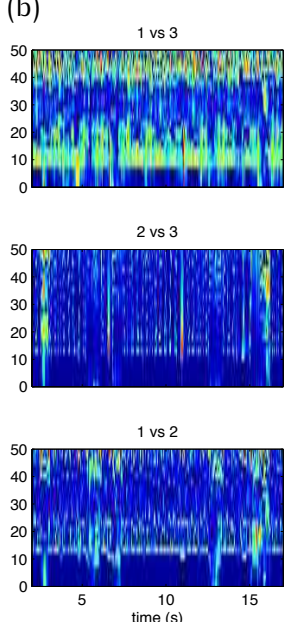

(f)

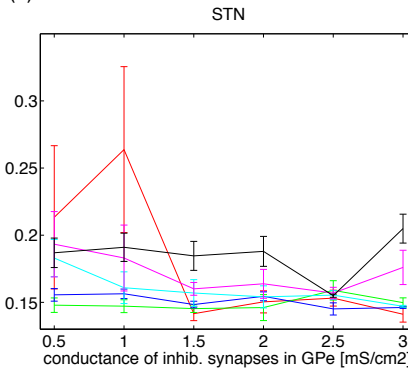

(i)

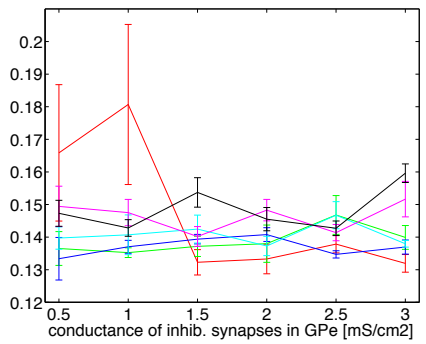

2 vs 3
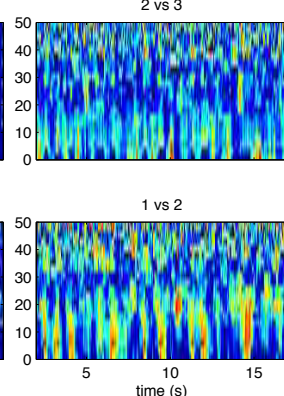

(d)
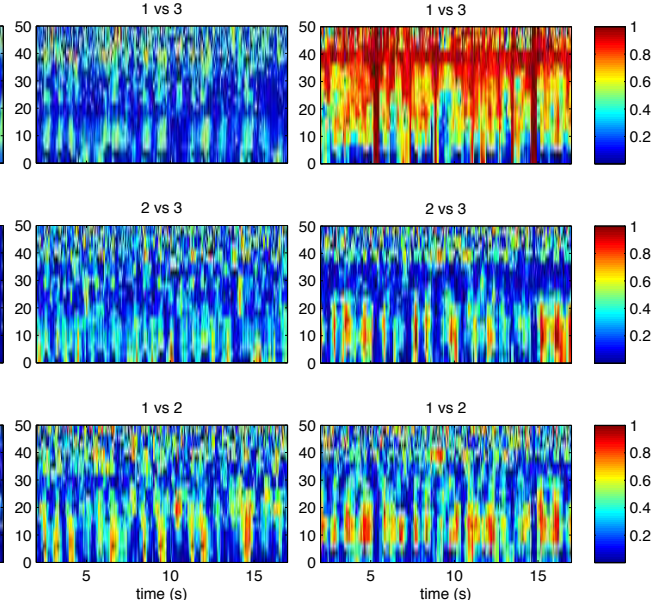

(g)

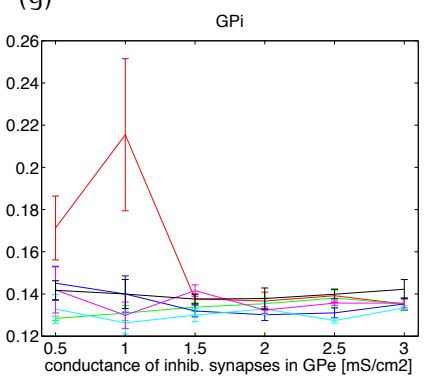

(j)

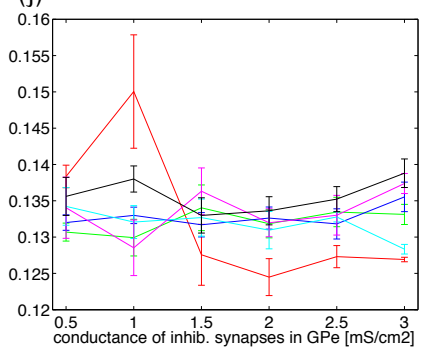

Figure 4.6: Coherograms for $80 \%$ synapse and gap junction exclusion: examples of coherence between cell pairs evolving over time (a) without, (b) with low $\left(0.1 \frac{\mathrm{mS}}{\mathrm{cm}^{2}}\right)$, (c) medium $\left(0.3 \frac{\mathrm{mS}}{\mathrm{cm}^{2}}\right)$ and (d) high $\left(0.5 \frac{\mathrm{mS}}{\mathrm{cm}^{2}}\right)$ gap junction coupling. (e-g) Average beta coherence in $C_{1} P e(e), S T N(f)$ and $C_{1} P i(g)$, and $(h-j)$ relative beta coherence (normalized by the total coherence) in (h) G.Pe, (i) STN and (j) G.Pi do not show clear trends towards more beta coherence with increasing gap junction coupling. 


\subsubsection{Effects already occurred in small model}

We also simulated a small network of 50 Rubin $\varepsilon_{r}$ Terman neurons (Fig. 4.7). Both synapses and gap junctions were excluded to $50 \%$. This small model essentially showed the same behavior as the full model. Fig. 4.7(a-c) depicts the number of principal components for GPe, STN and $\mathrm{G}_{1} \mathrm{Pi}$, respectively. As fewer connections in total existed in the small network compared to the full network, also variations were higher. Still, a desynchronization with increasing inhibition could be seen in $\mathrm{C}_{1} \mathrm{Pe}$ $(p<0.05$, see Section 4.2.3). Also slight gap junction coupling led to decorrelation $(p<0.05)$, whereas strong gap junction coupling synchronized the network $(p<0.01)$. Beta coherence and relative beta coherence (Fig. 4.7(d-i)) showed roughly the same behavior as the full model, namely an amplification of beta coherence with high gap junction coupling $(p<0.01)$. The effects of this beta amplification were not seen when gap junctions were very sparse (80\% exclusion, not shown). However, synchrony in general as quantified by the PCA was still dependent on both gap junctions and inhibition as in the full model. Since the behavior of the small model was very similar to the behavior of the full model including 500 cells at least when using Rubin $\varepsilon$ Terman neurons, we chose to simulate only 50 cells when using the computationally more expensive Fujita GPe neuron model.

\subsubsection{Similar results for Fujita neuron}

We also used a second neuron model, the Fujita neuron [Fujita et al., 2012], and simulated a small network of 50 cells. As shown in Fig. 4.8, the main results were similar to the small network with Rubin \& Terman neurons (Section 4.3.4). Beta coherence, but also relative beta coherence, increased with high gap junction coupling $(p<0.01$, see Section 4.2.3) when $50 \%$ of synapses and gap junctions were excluded (Fig. 4.8(a-f)). On the other hand, desynchronization by inhibition was less clear when using the Fujita neuron, possibly due to the only very short negative part of the PRC. Increasing inhibition had barely any effect on beta coherence when gap junction coupling was low, but could desynchronize for high gap junction strengths (Fig. 4.8(a-c)). The effects again were not significant when $80 \%$ of synapses and gap junctions were excluded (Fig. 4.8(g-l)). 
(a)

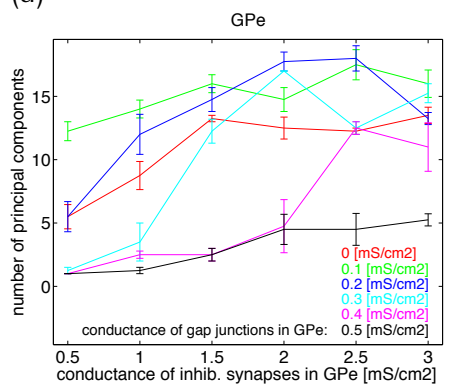

(d)

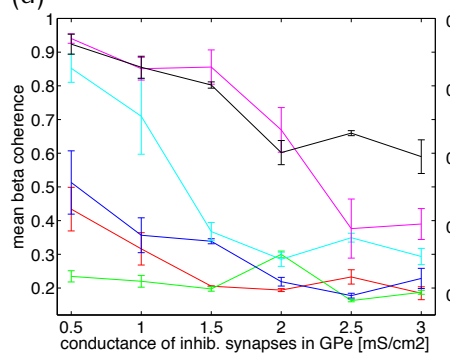

(g)

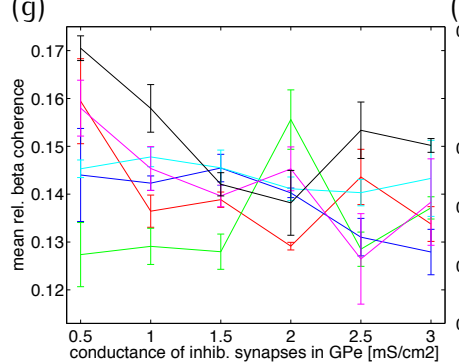

(b)

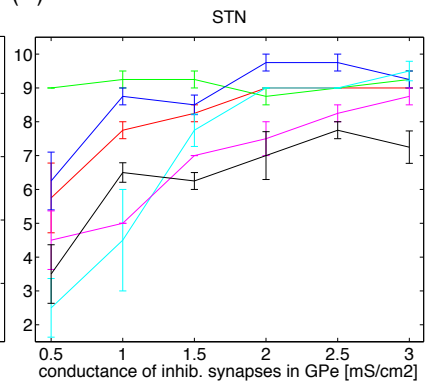

(e)

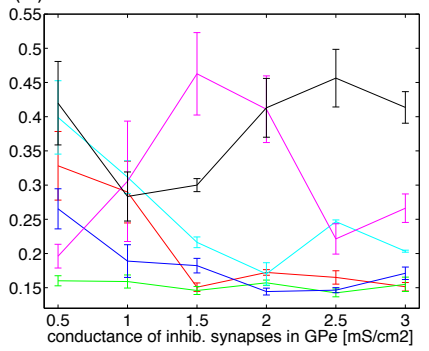

(h)

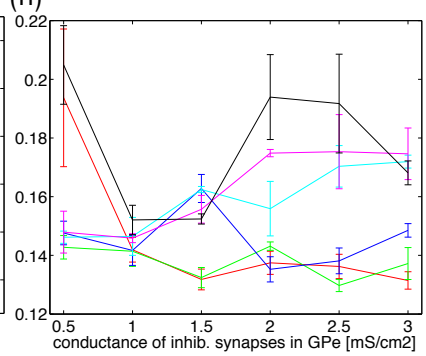

(c)

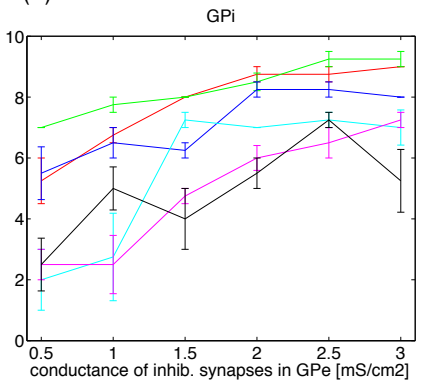

(f)

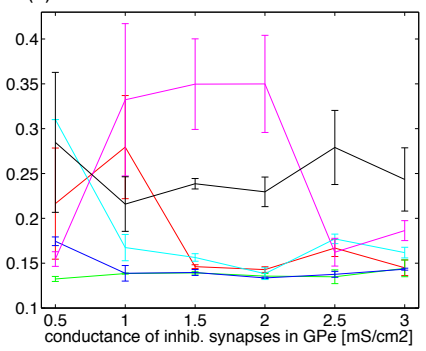

(i)

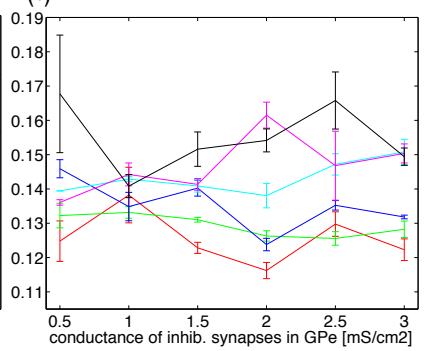

Figure 4.7: Main results for the small model that included 50 Rubin E Terman neurons, with 50\% synapse and gap junction exclusion: PCA analysis in GPe (a), STN (b) and $C_{1} P i$ (c). Mean beta coherence in $C_{1} P e(d), S T N(e)$ and $C_{1} P i(f)$, and relative beta coherence in $\mathrm{G}_{1} \mathrm{Pe}(\mathrm{g}), \mathrm{STN}(\mathrm{h})$ and $\mathrm{C}_{1} \mathrm{Pi}(\mathrm{i})$. The results were similar to the full model, but variations were stronger.

\subsection{Discussion and Conclusions}

In our network model, sparse inhibition between G.Pe cells was able to efficiently desynchronize neural activity in the whole basal ganglia. Additionally, sparse gap junction coupling of low strength boosted this desynchronization, leading to irregular firing in C.Pe already at low strengths of inhibitory synapses. On the other hand, stronger gap junction coupling, although still sparse, was able to synchronize activity. Notably, when gap junction coupling in GPe was sufficiently high, sustained 
(a)

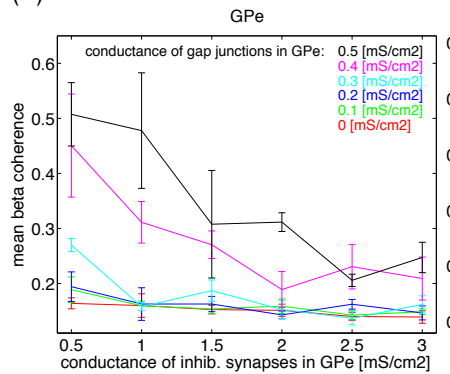

(d)

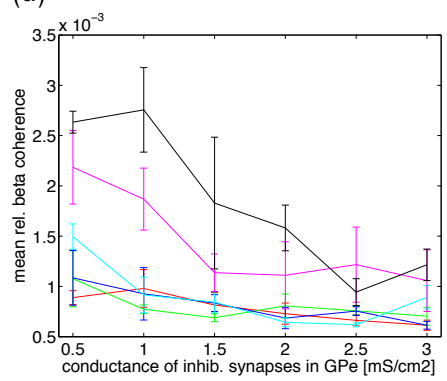

(g)

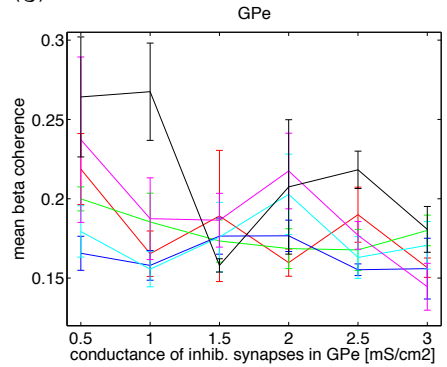

(j)

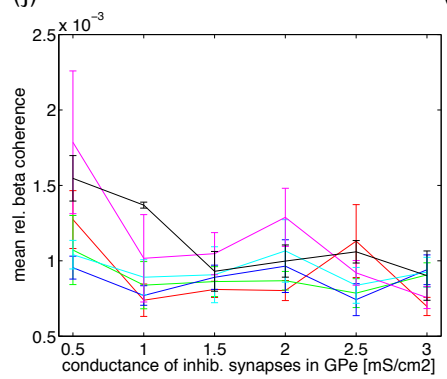

(b)

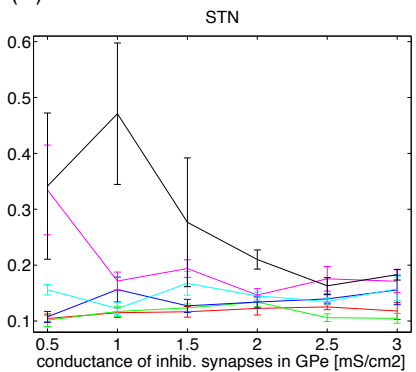

(e)

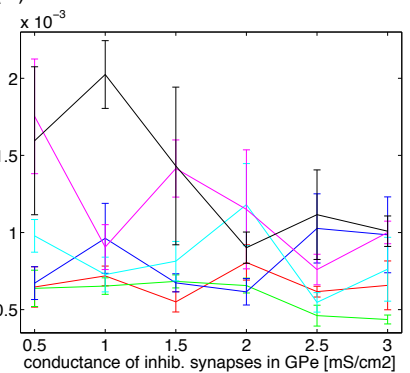

(h)

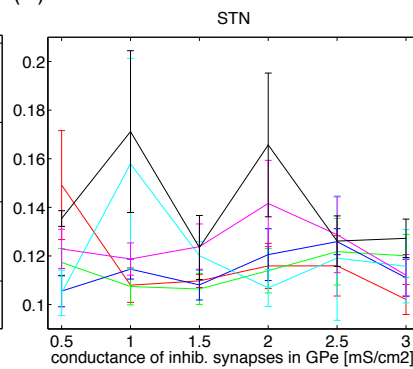

(k)

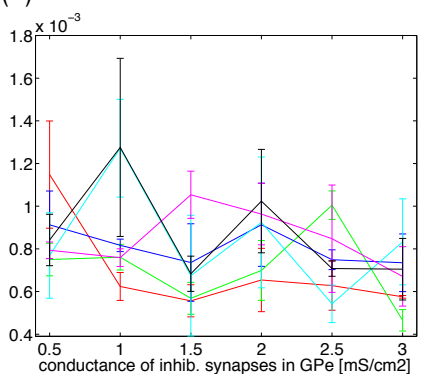

(c)

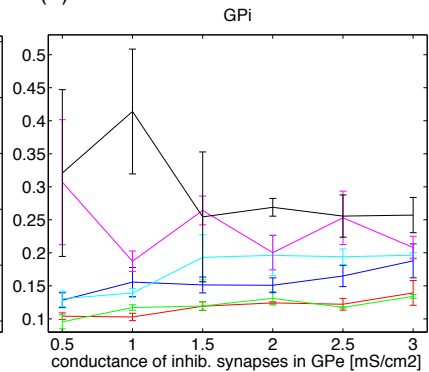

(f)

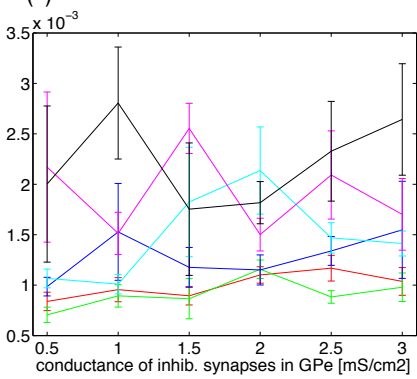

(i)

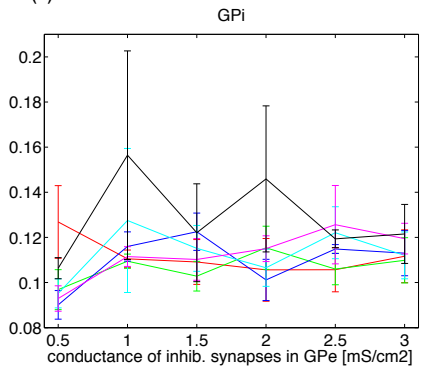

(l)

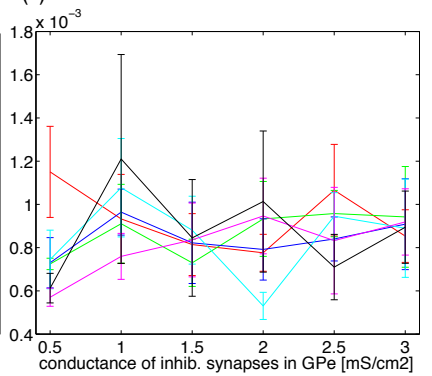

Figure 4.8: Main results for the small model with Fujita GPe neurons, (a-f) with $50 \%$ synapse and gap junction exclusion, $(\mathrm{g}-\mathrm{l})$ with $80 \%$ synapse and gap junction exclusion. $(a-c)$ and $(g-i)$ show the beta coherence, $(d-f)$ and $(j-l)$ the relative beta coherence. $(a, d, g, j)$ depict results for the $C_{1} P e,(b, e, h, k)$ for the STN and $(c, f, i, l)$ for the $\mathrm{C}_{\mathrm{P}} \mathrm{Pi}$. Both beta coherence and relative beta coherence increased with gap junction coupling if the connectivity was not very sparse. 
epochs of synchronized firing at low frequencies occurred in some neuron pairs of all simulated nuclei. As many neurons were spiking with frequencies around the beta band, it is not very surprising that gap junctions led more often to synchronous firing at beta frequency than at other frequencies. However, it is remarkable that sparse gap junction coupling in $\mathrm{C}_{1} \mathrm{Pe}$ is sufficient to induce this synchronization in $\mathrm{C}_{1} \mathrm{Pe}$ as well as in $\mathrm{CPi}_{\mathrm{P}}$ and STN. We suggest that, if gap junctions are present in $\mathrm{CP}_{\mathrm{Pe}}$, the modulation of gap junction conductance by dopamine could contribute to the shifts in synchronous basal ganglia activity seen in PD patients.

We acknowledge several limitations of this study. First of all, our network is a highly simplified description of the nuclei STN, G.Pe and GPi. We used single compartment cell models, neglecting for example dendrites. Gap junctions might in particular occur at dendritic contacts, as well as on axons or somata [Witkovsky et al., 1983]. Such coupling via dendrites could weaken the roles of gap junctions, since an additional resistance is located between gap junction and soma. Also dendritic conduction delays could occur which have been omitted in our model. Further, connectivity within and between nuclei was only rudely estimated. We did not model striatum itself but only input from striatum to G.Pe. However, it has become apparent that G.Pe also densely inhibits striatum [Fujiyama et al., 2015], building the basis for a feedback loop between these two nuclei. Moreover, we modeled only one GPe population, although it is known that several types of C.Pe neurons, differing in both structure and function, exist [Mallet et al., 2012, Mastro et al., 2014, Dodson et al., 2015, Abdi et al., 2015]. Despite all this neglected detail and heterogeneity, we suggest that our model has biological relevance. We could show that, for example, the concrete connection architecture had only a minor influence on the development of synchrony. Hence, we propose that the mechanism, namely control of synchrony by sparse gap junction coupling, is robust with respect to this detail.

Gap junctions have often been related to synchrony and oscillations [Draguhn et al., 1998, Tamás et al., 2000]; in particular, recently also in the context of PD [Traub and Whittington, 2010, Phookan et al., 2015]. While an early study showed first evidence of gap junctions in the rodent GP by using electron microscopy [Kita, 1994], and also Cx36 was reported in the rodent G.P [Rash et al., 2000], we described the presence of $\mathrm{C} 36$ in the human G.Pe and C.Pi [Schwab et al., 2014]. In our previous work [Schwab et al., 2014], we showed only a very small and basic computational model of the basal ganglia, which is worked out here in detail. The idea that pallidal gap junctions contribute to synchrony in the parkinsonian basal ganglia has also been brought up by Phookan et al. [2015], who used broad-band gap junction blockers in a 6-hydroxydopamine (6-OHDA) rat model of parkinsonism. Both sys- 
temic and local injection of carbenoxolone or octanol into G.Pe led to an attenuation of beta oscillations, and even an improvement of akinesia in a step test. This is in line with our model, suggesting that a decrease in pathologically high gap junction conductance leads to a decrease in synchrony and hindered decorrelation of beta input. However, it is not known to which extent the effects seen by Phookan et al. [2015] are due to blocking of neural gap junctions, as carbenoxolone and octanol also act on gap junctions between glial cells.

Therefore, to validate our model, Cx36 knock-out (KO) mice would be ideally suited. If gap junctions between pallidal neurons play a role in PD pathology, beta oscillations of such $\mathrm{KO}$ mice should show attenuated beta oscillations and less synchrony in the basal ganglia than wild type animals. As Cx36 seems to be solely expressed by neurons [Rash et al., 2000], this experiment would be able to distinguish the influence of neural and glial gap junctions. Astrocytes, on the other hand, are abundant in C.Pe [Lange et al., 1976] and could also influence synaptic transmission [Galvan et al., 2005, 2010, Gittis et al., 2014]. In a rotenone rat model of PD, Cx43, mainly expressed by astrocytes, was increased in C.Pe compared to control rats, possibly as a sign of inflammation [Kawasaki et al., 2009]. Many factors might contribute to altered activity in the basal ganglia, among them possibly a loss of neural pacemaking function [Chan et al., 2011] and synaptic changes (e.g., Fan et al. [2013], Miguelez et al. [2012], Chu et al. [2015]). Since gap junctions show fast functional changes dependent on, for example, neurotransmitter concentrations, $\mathrm{pH}$, or even activity [Spray et al., 1981, Haas et al., 2011, Li et al., 2013, Palacios-Prado et al., 2013], they also might be ideally suited to take part in inducing pathological activity shifts. Nevertheless, the occurrence and modification of gap junctions in the basal ganglia, including striatum, have barely been studied yet. 


\title{
Dynamics of the basal ganglia-thalamic connection during movement
}

\begin{abstract}
The basal ganglia are considered as crucial for motor control, and movement disorders such as Parkinson's disease come along with altered basal ganglia activity. However, it is unclear how the basal ganglia exert their influence on thalamic nuclei and successively on premotor and motor cortices, and thereby contribute to the execution of learned and novel movements. Most theories state that the output stages of the basal ganglia inhibit or disinhibit thalamic firing, leading to modulation or gating of thalamic activity, which is then relayed to cortical areas.

To test this assumption, we recorded single unit activity and local field potentials (LFPs) in-vivo from the output stage of the basal ganglia, the internal globus pallidus $\left(\mathrm{C}_{1} \mathrm{Pi}\right)$, and the basal ganglia input stage of thalamus, the ventrolateral anterior nucleus (VLa), in one monkey performing a choice reaction time reaching task. Recorded neurons from $\mathrm{C}_{1} \mathrm{Pi}$ and VLa were part of a functional loop including the primary motor cortex (M1). The data were complemented by M1 LFP recordings from implanted electrodes in the same monkey and by both single unit and LFP recordings from movable electrodes in another monkey performing the same task.

Neural activity was qualitatively different in C.Pi compared to M1, lacking spike correlations during both rest and movement periods. Inconsistent with the theory that basal ganglia activity entrains thalamic firing, we found only very weak spike correlations between $\mathrm{C}_{1} \mathrm{Pi}$ and VLa that were furthermore not altered during movement. When combining information of all single unit data from C.Pi and VLa, spike counts showed subtle excitatory correlations for C.Pi leading VLa, and subtle inhibitory correlations when VLa led $\mathrm{C}_{1} \mathrm{Pi}$. Nevertheless, LFP correlations between these nuclei were strong, and could only scarcely be explained by a common cortical influence. Spike-LFP correlations were lower in $\mathrm{C}_{1} \mathrm{Pi}$ than in M1. As correlations between $\mathrm{C}_{1} \mathrm{Pi}$ spikes and VLa LFPs were virtually absent, we were not able to demonstrate a feed-
\end{abstract}


forward connection from CIPi to VLa.

Our results suggest that processing of movement-related information in subcortical structures differs from cortical processing. We further conclude that the interaction of the basal ganglia with thalamus during learned movements is more complicated than previously assumed, potentially involving weak connections between large numbers of neurons. 1

1 The study described in this chapter is part of a collaboration with Jonathan E. Rubin and Robert S. Turner at the University of Pittsburgh, PA, USA, and has not been published yet. We describe here the core findings using preliminary data of one monkey, complemented by cortical data from another monkey. Data collection from more animals, including parkinsonian monkeys, is ongoing. The experimental part of this work as well as preprocessing of the data were carried out by Andrew J. Zimnik and Robert S. Turner, University of Pittsburgh, PA, USA. 


\subsection{Introduction}

The basal ganglia consist of segregated reentrant loops, arising in motor cortex, and projecting back to motor cortex via thalamic nuclei [Alexander et al., 1986, Graybiel et al., 1994]. Movement disorders, especially Parkinson's disease (PD), are often mentioned to have their origin in abnormalities of these nuclei since their motor symptoms correlate with characteristic changes in basal ganglia activity (for review, see Galvan et al. [2015]). It has been suggested that an increase in bursting, the presence of slightly altered firing rates as well as synchronous and oscillatory firing, are of pathophysiological relevance as these changes might affect thalamic ability to relay cortical inputs [Brown, 2003, Rubin et al., 2012, Galvan et al., 2015].

Surprisingly little is known on how the basal ganglia contribute to skeletal movement under physiological conditions. In contrast to cortical activity, basal ganglia output, measured in the internal globus pallidus ( $\left.\mathrm{C}_{1} \mathrm{Pi}\right)$, does not show a clear linear relationship with basic movement parameters such as movement amplitude or movement velocity [Brotchie et al., 1991, Mink and Thach, 1991]. Nevertheless, basal ganglia output discharge is highly influenced by movement kinematics [Turner and Anderson, 1997]. It has thus been suggested that the basal ganglia regulate the amount of movement rather than directly control it [DeLong and Wichmann, 2007]. During the last years, a number of studies have investigated the influence of basal ganglia activity downstream in order to estimate their influence on movement under physiological and pathological conditions (for review, see Rubin et al. [2012], BoschBouju et al. [2013]).

Different theories have been proposed for the healthy brain: One theory states that the basal ganglia normally inhibit motor thalamus, and gate thalamic activity via disinhibition [Horak and Anderson, 1984, Deniau and Chevalier, 1985, Chevalier and Deniau, 1990, Nambu et al., 1991, Nambu, 2004, Hikosaka, 2007]. Within the disinhibitory phases, cortex would drive thalamic activity. In another theory, the inhibitory influence of the basal ganglia allows only very small, precise windows of thalamic firing, thereby leading to an anti-phasic driving of thalamus by GPi activity [Goldberg et al., 2012, Goldberg and Fee, 2012]. Finally, it has been proposed that inhibition by the basal ganglia can trigger rebound low threshold spike (LTS) bursts in thalamus [Person and Perkel, 2005, 2007, Bosch-Bouju et al., 2013, 2014]. These bursts might occur after basal ganglia inhibition decreases, thereby effectively driving thalamic activity [Bosch-Bouju et al., 2013]. 
In this study, we recorded basal ganglia output activity ( $\left.\mathrm{C}_{1} \mathrm{Pi}\right)$ as well as activity in the basal ganglia-receiving territories of motor thalamus (ventrolateral anterior nucleus, VLa) in order to investigate information transmission between these two structures relevant for motor control. We compared dynamics of single unit activity and local field potentials (LFPs) in GPi and VLa to cortical dynamics during a simple, highly-learned movement task. Spike dynamics were of a different nature in $\mathrm{C}_{1} \mathrm{Pi}$ than in primary motor cortex (M1). Based on single unit data, we could not confirm the theory of entrainment or gating of VLa by $C_{1} P i$, but saw only very slight and delayed influences between these structures. In contrast, LFPS in both GPi and VLa correlated with cortical LFPs and also with each other, although M1 seemed not to be the main driver of the $\mathrm{C}_{1} \mathrm{Pi}-\mathrm{VLa}$ correlations. Our results therefore suggest that large numbers of neurons are necessary to transmit information from basal ganglia to thalamus.

\subsection{Methods}

\subsubsection{Experimental setup and data preprocessing}

One female monkey $\left(C_{1}\right)$ and one male monkey $(B)$, both Macaca mulatta, were used in this study. All aspects of animal care were in accord with the National Institutes of Health Guide for the Care and Use of Laboratory Animals, the PHS Policy on the Humane Care and Use of Laboratory Animals, and the American Physiological Society's Guiding Principles in the Care and Use of Animals. All procedures were approved by the institutional animal care and use committee. Surgery was performed as described in Zimnik et al. [2015] and Zimnik and Turner [2015]. The data set from monkey $C_{1}$ is identical to the data presented by Zimnik and Turner [2015].

In monkey $\mathrm{G}_{1}$, LFPs were recorded from each two movable electrodes in the internal part of the globus pallidus ( $\mathrm{C} P \mathrm{Pi}$ ) and the ventrolateral anterior nucleus (VLa) as well as from one implanted electrode in the subthalamic nucleus (STN) and three implanted electrodes in the distal arm region, the elbow region, and the face region of the primary motor cortex (M1). Further, single unit data was obtained from the four movable electrodes in C.Pi and VLa. In monkey B, both LFP and spike train data were recorded from movable electrodes in M1. An overview of these electrode positions is given in Table 5.1. All electrodes were referenced to a dura-piercing cannula.

Both monkeys performed a highly trained reaching task as in Franco and Turner [2012], Zimnik et al. [2015], and Zimnik and Turner [2015]. In short, the monkeys were 
seated in front of two visual targets (LEDs) and held their hands for $2.6-4.8 \mathrm{~s}$ at the home keys. When one of the targets was illuminated, the animals had to hold their hand at this target for $0.5-1.0 \mathrm{~s}$ before a reward was delivered. Thereafter, the animals could return their hand to the home-key without a time limit. Holding times and selection of the targets were randomised so that the animals were not able to predict the time of an upcoming stimulus.

\begin{tabular}{c|c|c|c} 
& LFP (movable electrodes) & LFP (implanted electrodes) & Single unit (movable electrodes) \\
\hline $\mathrm{C}_{1} \mathrm{Pi}$ & $\mathrm{G}_{1}$ & & $\mathrm{G}_{1}$ \\
\hline $\mathrm{VLa}$ & $\mathrm{G}_{1}$ & & $\mathrm{G}_{1}$ \\
\hline $\mathrm{M} 1$ & $\mathrm{~B}$ & $\mathrm{C}_{1}$ & $\mathrm{~B}$ \\
\hline $\mathrm{STN}$ & & $\mathrm{G}_{1}$ &
\end{tabular}

Table 5.1: Overview of the recorded electrodes in monkey $G$ and $B$.

Data acquisition and preprocessing has partly been reported in McCairn and Turner [2009], Zimnik et al. [2015], and Zimnik and Turner [2015], and is only summarized briefly here. All signals were amplified $(x 4)$. Signals from implanted electrodes were acquired at $1017.3 \mathrm{~Hz}$ resolution and filtered between 2 and $400 \mathrm{~Hz}$. Signals from movable electrodes were acquired at $25 \mathrm{kHz}$ resolution before wide-band filtering $(2 \mathrm{~Hz}$ to $7.5 \mathrm{kHz})$. Offline, the data from movable electrodes were low pass filtered $(<400 \mathrm{~Hz})$ to obtain local field potentials (LFPs), and high pass filtered $(>200 \mathrm{~Hz})$ to obtain single unit activity. The single unit data were thresholded and candidate action potentials were sorted into clusters by principal component analysis using the commercial software Plexon.

For all analyses, the LFP and single unit data were down-sampled to $1 \mathrm{kHz}$ resolution. The single unit data consisted of binary time series indicating a spike (1) or no spike (0). Only paired recordings with at least one single unit signal in both $\mathrm{C}_{1} \mathrm{Pi}$ and VLa were included in the analysis. Preliminary data used in this study consisted in total of 330 units 606 LFP signals in monkey $C_{1}$ as well as 327 units and 134 LFP signals in monkey $\mathrm{B}$.

\subsubsection{Verification of functional circuitry}

Zimnik and Turner [2015] confirmed that the recorded areas belonged to a functional circuit between basal ganglia, thalamus and cortex. Macroelectrodes were implanted 
chronically into the superior cerebellar peduncle (SCP) and arm-related locations of M1. VLa neurons were orthodromically activated by M1 stimulation, but not excited from SCP. M1 stimulation further led to responses in $\mathrm{C}_{1} \mathrm{Pi}$, possibly mediated via STN. Finally, stimulation of $\mathrm{C}_{1} \mathrm{Pi}$ by movable electrodes could in one recording completely inhibit spiking in VLa with short latencies of $1-4 \mathrm{~ms}$. Although most neurons in $\mathrm{C}_{1} \mathrm{Pi}$ and VLa responded to M1 stimulation, some did not. It is therefore possible that a fraction of the recorded data was not part of one basal ganglia-thalamo-cortical pathway.

\subsubsection{Noise correlations}

To estimate whether the spike train data contained common times of discharge, e. g. during movement, we calculated noise correlations of the whole recordings. The number of spikes in $10 \mathrm{~ms}$ time bins were counted for every spike train. Each spike count pair $\{x, y\}$ was assigned a Pearson's coefficient $\rho(x, y)$ :

$$
\rho(x, y)=\frac{1}{N-1} \sum_{i=1}^{N} \frac{x_{i}-\bar{x}}{\sigma_{x}} \frac{y_{i}-\bar{y}}{\sigma_{y}} \in[-1,1]
$$

where $N$ is the number of time bins considered, $x_{i}$ and $y_{i}$ are the spike count value at time bin $i$ for each unit, $\bar{x}$ and $\bar{y}$ are the mean spike counts of $x$ and $y$, respectively, and $\sigma_{x}$ and $\sigma_{y}$ their respective standard deviations. A Pearson's coefficient of 1 indicates a perfect positive correlation, -1 a perfect negative correlation. Note that this analysis was done without correcting for movement-related discharge. We also performed the same analysis with shuffled spike times, leading to a control distribution of Pearson's coefficients.

\subsubsection{Detection of movement-related discharge}

Similar as in Zimnik and Turner [2015], we studied discharge of single unit data around movement onset. The data were segmented into windows of $800 \mathrm{~ms}$ before and $800 \mathrm{~ms}$ after movement onset. All spike trains were convolved with a Craussian kernel of standard deviation $20 \mathrm{~ms}$ and unit area, and averaged over all trials of one single unit recording. The period of $-700 \mathrm{~ms}$ to $-200 \mathrm{~ms}$ relative to movement onset was used as a baseline. A cell was classified as "increasing", if the firing rate increased in the time range $-200 \mathrm{~ms}$ to $200 \mathrm{~ms}$ relative to movement onset with more than 3.1 standard deviations of the baseline. 3.1 standard deviations were chosen 
as they relate to a $99.9 \%$ confidence interval. Likewise, the cell was classified as "decreasing", if the firing rate dropped more than 3.1 standard deviations, and it was classified as "increasing $\varepsilon$ decreasing" if it fulfilled both of the latter criteria. All remaining cells were classified as "constant". Based on the obtained groups, we averaged the obtained spike densities around movement onset.

\subsubsection{Spike and LFP correlations}

The spike trains, consisting of unit pulses, were convolved with a Gaussian kernel of standard deviation $2 \mathrm{~ms}$ and unit area. Both single unit and LFP data were divided into 200 ms segments relative to the behavioral events cue onset, movement initiation, time of reward, and return movement initiation (see Table 5.2). For each of these segments, the normalized cross-correlation function (CCF) between two signals was computed separately and for both unit and LFP data:

$$
\begin{aligned}
\operatorname{CCF}_{x, y}(m) & =\frac{1}{R} \sum_{n=0}^{N-m-1} x_{n+m} y_{n} & & \text { for } m \geq 0 \\
& =\frac{1}{R} E\left[x_{n+m} y_{n}^{*}\right] & & \\
\operatorname{CCF}_{x, y}(m) & =\operatorname{CCF}_{x, y}(-m) & & \text { for } m<0
\end{aligned}
$$

where $\{x, y\}$ is the signal pair. We normalized the CCFs with $R=\sum_{n=0}^{N-1} x_{n} y_{n}$. Autocorrelations therefore have a peak value of 1 at $m=0$ and $C C F_{x, y} \in[-1,1]$.

Thereafter, the CCFs were averaged over all trials within one recording. Thus, we obtained one averaged CCF per recording for each time window relative to an event, e.g., after movement initiation. From those averaged CCFs, we stored both the peak value of the absolute value of the CCF and the location (time lag) of this peak. For spike-spike correlations within C.Pi, VLa or M1, we used only positive peak values of the CCF to avoid artifacts due to the shadowing effect. Shuffling all spike times before convolution with a gaussian and all LFP data at $1 \mathrm{~ms}$ resolution, we obtained control correlations for both spike and LFP CCFs. We detected the maxima of those distributions in the same way as for the actual CCFs. 


\subsubsection{Prediction of LFP correlations by a third LFP signal}

To estimate whether any of our LFP correlations resulted from the common linear influence of a source, namely a third LFP signal, we conducted a multivariate analysis [Halliday and Rosenberg, 1999]. As for the direct calculation of correlations, LFP signals were cut into segments of $200 \mathrm{~ms}$ relative to events. For each segment, we calculated the "predicted CCF" (pCCF)

$$
{ } C C F_{L_{i}, L_{j}, S}=\mathcal{F}^{-1}\left(\frac{\mathcal{F}\left(C C F_{L_{i}, S}\right) \mathcal{F}\left(C C F_{S, L_{j}}\right)}{\left|\mathcal{F}\left(C C F_{S, S}\right)\right|}\right),
$$

characterising the correlation between two LFP signals $x$ and $y$ arising from the common linear effect of the third signal $S$. $\mathcal{F}$ and $\mathcal{F}^{-1}$ indicate the forward and inverse Fourier transform, respectively. As the CCF is normalized, also the pCCF peak values are $\in[-1,1]$. The pCCF can be compared to the directly calculated CCF. $A$ motivation of Equation (5.5) is given in Goldberg and Bergman [2005] and summarized in the appendix, based on Priestley [1981] and Percival and Walden [1993]. In short, we assume that all LFPs underlie stationary processes. The two LFPs $L_{i}$ and $L_{j}$ are assumed to have a linear relationship to the source LFP $S$. Formal re-computation of the CCF between $L_{i}$ and $L_{j}$, both clescribed by the convolution of an arbitrary function $\left(a_{i}\right.$ and $\left.a_{j}\right)$ with $S$, leads to the pCCF.

When both denominator and numerator become very small, the pCCF has a low signal-to-noise ratio. Therefore, for frequencies where the power of both the denominator and the numerator were marginal ( $<10 \%$ of the mean LFP power), the power of the predicted spectrum was set to zero. Still, autocorrelations were without loss predicted by their own LFP. Further processing was done as described in Section 5.2.5. The pCCFs were again averaged over all trials of one recording, and their peak value and the time lag of the peak were stored. Finally, corrected LFP correlations (cCCFs) were calculated by subtraction of the predicted CCFs from the directly calculated CCFs:

$$
\mathrm{cCCF}_{x, y}(m)=\left|\mathrm{CCF}_{x, y}(m)\right|-\left|p C C F_{x, y, S}(m)\right| .
$$

Fig. 5.1(a) depicts an example of a CCF which is partly predicted by a third LFP signal.

The shuffled controls consisted of two original LFP signals and one shuffled source signal. The shuffled source signal is not expected to predict nonzero correlations, thus the correlations predicted by the shuffled source were used as the control. Peaks of 
those controls were detected the same way as for the original data, and plotted as control distributions.

(a)

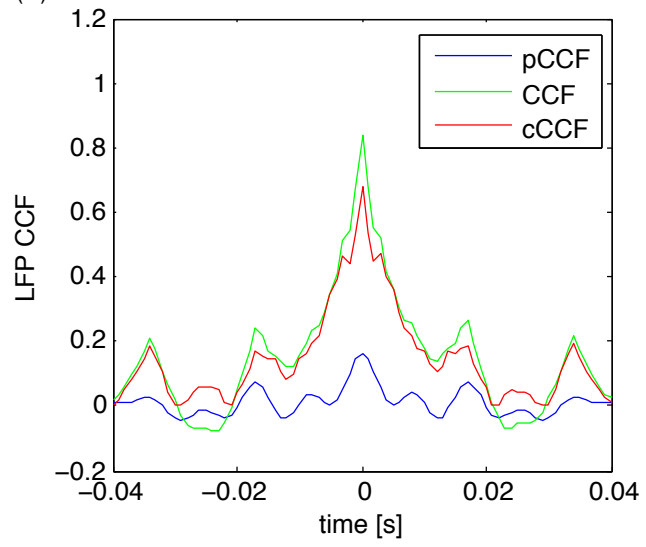

(b)

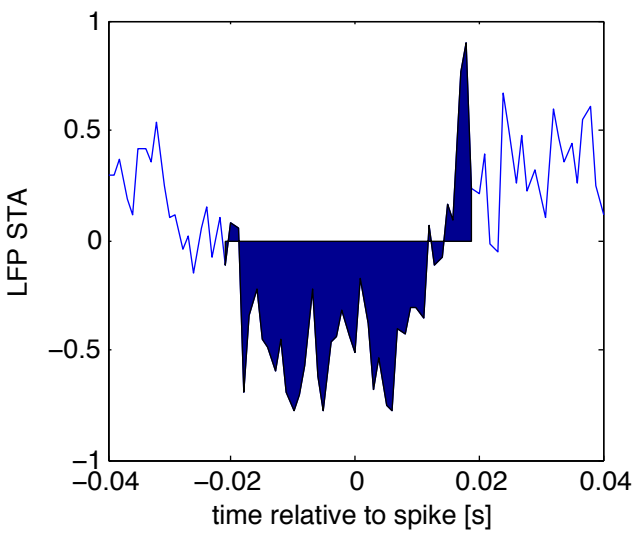

Figure 5.1: (a) Example of an LFP CCF (CCF), its prediction by another LFP (pCCF), and the corrected CCF (cCCF). (b) Example of an LFP STA, averaged over all trials of one recording. Values of the STA within an interval of $20 \mathrm{~ms}$ before and after the spike (filled area) were integrated to obtain one value for spike-LFP coupling.

\subsubsection{Spike-LFP coupling}

We used two different measures to assess phase coupling between spikes and LFP data. All data were again cut into $200 \mathrm{~ms}$ windows relative to behavioral events (see Table 5.2). Both measures were computed separately for each $200 \mathrm{~ms}$ window and then averaged over all trials of one movement.

First, we calculated spike triggered averages (STAs) of the LFP data $X(t)$ as in Cioldberg et al. [2004]:

$$
\mu_{s}(\tau)=\frac{\frac{1}{N_{s}} \sum_{t_{s}} X\left(t_{s}+\tau\right)}{\sigma}
$$

where $t_{s}$ denotes the spike times and $\sigma$ is the standard deviation of $X(t)$. An example LFP STA, averaged over all trials of one recording, is shown in Fig. 5.1(b). Finally, we integrated the STA values over all time delays between -20 ms and $20 \mathrm{~ms}$ relative to the spike time to obtain the correlation coefficient. STAs that are equally positive and negative within this interval thus lead to a small correlation coefficient 
in this approach. To each pair of spike and LFP data, one correlation coefficient was assigned for each event window. Control STAs were generated by shuffling spike times, and processing the resulting control STAs equally as described before.

Second, we estimated whether spikes occured preferentially at a certain LFP phase of a certain LFP rhythm. For this, we filtered the LFP signal with a second order Butterworth bandpass filter in $5 \mathrm{~Hz}$ intervals between 5 and $45 \mathrm{~Hz}$. The filtered LFP signal $X(t)$ was then Hilbert transformed

$$
H[X(\tau)]=\frac{1}{\pi} P V \int_{-\infty}^{\infty} \frac{X(t) d t}{t-\tau}
$$

where PV denotes the Cauchy principal value. The phase of $H[X(t)]$ served as the phase of our filtered signal. We counted the number of spikes that occurred in a certain phase interval, and averaged these counts over all trials and recordings. Control distributions were calculated by shuffling all spike times and doing the same analysis. $95 \%$ confidence intervals were set to two standard deviations of the control distribution.

\subsection{Results}

We analysed single unit and LFP data as described in Section 5.2 during $200 \mathrm{~ms}$ windows relative to behavioral events. Table 5.2 summarizes our choice of those windows. Outward and return movements were both of durations longer than $200 \mathrm{~ms}$. "Movement initiation" refers to the time when the monkey limb passed a light beam, and might therefore be some ms after actual movement onset. Respectively, the time window "directly before movement" might include the actual movement onset. For most of the results, the time windows "after movement" and "after return movement" were neglected here as they did not show clear differences to other time windows.

To avoid volume conduction effects, all LFP analyses were done only with bipolar LFPs, i.e. using an electrode in the same nucleus as the reference. Since the STN included at most one electrode, bipolar LFPs were not available for the STN and are therefore not included in this chapter. As the preliminary clata presented here is limited, we cannot make a final statement yet about the significance of the results. This is in particular the case for differences between histograms in Sections 5.3.45.3.6. 


\begin{tabular}{l|l} 
before movement & $-400 \mathrm{~ms}$ to $-200 \mathrm{~ms}$ relative to outward movement initiation \\
\hline directly before movement & $-200 \mathrm{~ms}$ to $0 \mathrm{~ms}$ relative to outward movement initiation \\
\hline during movement & $0 \mathrm{~ms}$ to $200 \mathrm{~ms}$ relative to outward movement initiation \\
\hline during return movement & $0 \mathrm{~ms}$ to $200 \mathrm{~ms}$ relative to return movement initiation \\
\hline after cue onset & $0 \mathrm{~ms}$ to $200 \mathrm{~ms}$ relative to cue onset \\
\hline after reward & $0 \mathrm{~ms}$ to $200 \mathrm{~ms}$ relative to reward onset \\
\hline after movement & $0 \mathrm{~ms}$ to $200 \mathrm{~ms}$ relative to movement offset \\
\hline after return movement & $0 \mathrm{~ms}$ to $200 \mathrm{~ms}$ relative to return movement offset
\end{tabular}

Table 5.2: Time windows relative to behavioral events.

\subsubsection{Basic properties of recorded activity}

To introduce the analyzed data, we present several basic properties of the obtained single unit and LFP data in this section. Mean firing rates, averaged over whole recordings independent of movement, were higher in C.Pi than in M1 and VLa (Fig. 5.2(a); $p<0.001$, two-sample t-test). However, the rates varied highly between the recorded units. We detected bursts with the "surprise" method of Legendy and Salcman [1985] and its implementation by Wichmann and Soares [2006] with a surprise value of 4 , a minimum of 3 spikes per burst and at least a doubled frequency within bursts compared to the preceding $500 \mathrm{~ms}$ of firing. Bursts occurred in both $\mathrm{C}_{\mathrm{P}} \mathrm{Pi}$ and VLa, (Fig. 5.2(b)), but were more common in VLa (with respect to both the fraction of spikes and time in bursts, $p<0.005$, two-sample t-test). With the described parameters, we did not find bursts in the M1 data.

Using the LFP data from all movable electrodes, we examined whether the average LFP spectra changed during movement (Fig. 5.3). Fig. 5.3(a) shows an example spectrogram of a C.Pi LFP recording during one trial. The spectrogram was calculated using segments of $96 \mathrm{~ms}$ and an overlap of $60 \mathrm{~ms}$. Frequencies below about $10 \mathrm{~Hz}$ are thus not captured in this spectrogram. Bursts at high beta frequency with lengths of around 20-200 ms are clearly visible. When averaging over all trials and recordings, these bursts were lost and only expected values of beta power stayed. Before averaging, all spectra were normalized with the spectrum of the time window "before movement onset". Deviations between recordings were high, indicated by error bars (standard error of the mean, SEM).

In all M1, GPi and VLa, LFP power in general was higher during movement compared to the rest period, and especially huge in C.Pi. Both C.Pi and VLa showed peaks at high beta frequency $(20-30 \mathrm{~Hz})$. In VLa, these peaks were clearest during 
(a)

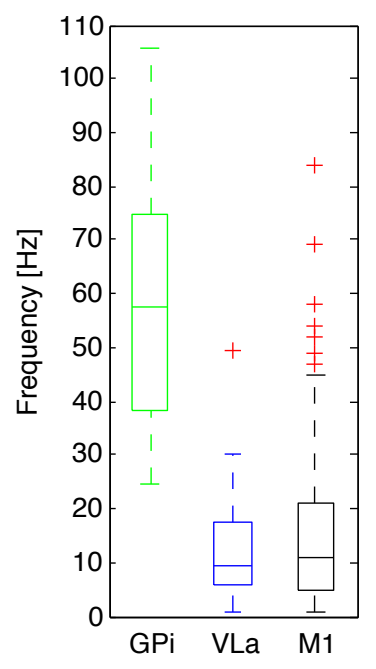

(b)

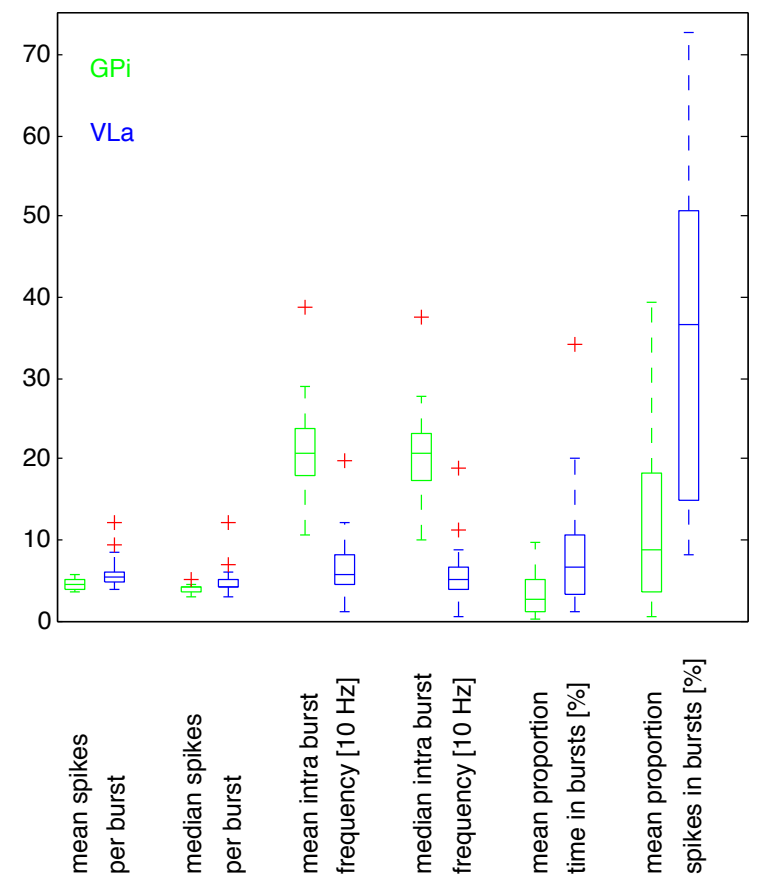

Figure 5.2: (a) Firing rates averaged over all recordings in C.Pi, VLa and M1. (b) Burst parameters in $\mathrm{CPi}$ and VLa. Although mean firing rates were much higher in $\mathrm{C}_{1} \mathrm{Pi}$ than in VLa, bursts were more common in VLa than in $\mathrm{C}_{1} \mathrm{Pi}$. No bursts were detected in M1. Central marks indicate the median, and box edges the 25th and 75th percentile. The dashed whiskers extend to the smallest and largest data points, excluding outliers which are plotted individually as red crosses. Square brackets at the end of a property indicate the scaling of the $y$-axis.

return movement and after reward. We summed the power at beta frequencies (12$30 \mathrm{~Hz}$ ) for each time window and divided this beta power by the total power of each spectrum, leading to the relative beta power (Fig. 5.3(e)). Relative beta power in $\mathrm{C}_{1} \mathrm{Pi}$ was significantly decreased during movement compared to the resting period $(p<0.0001$; two-sample t-test). In both VLa and M1, the relative beta power already dropped significantly directly before movement ( $p<0.0001$, two-sample t-test). 
(a)

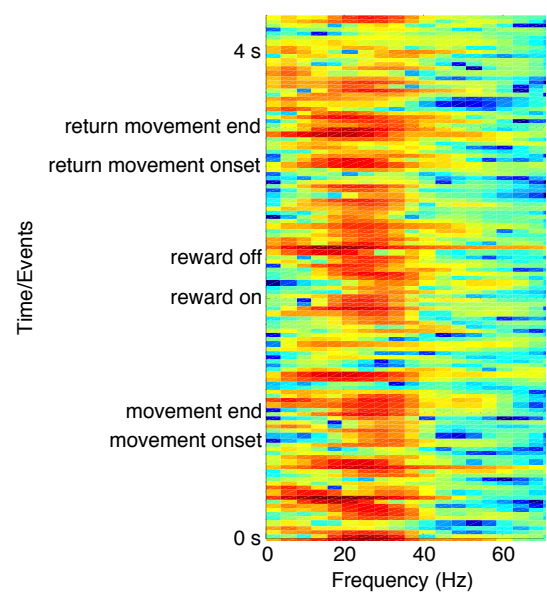

(c)
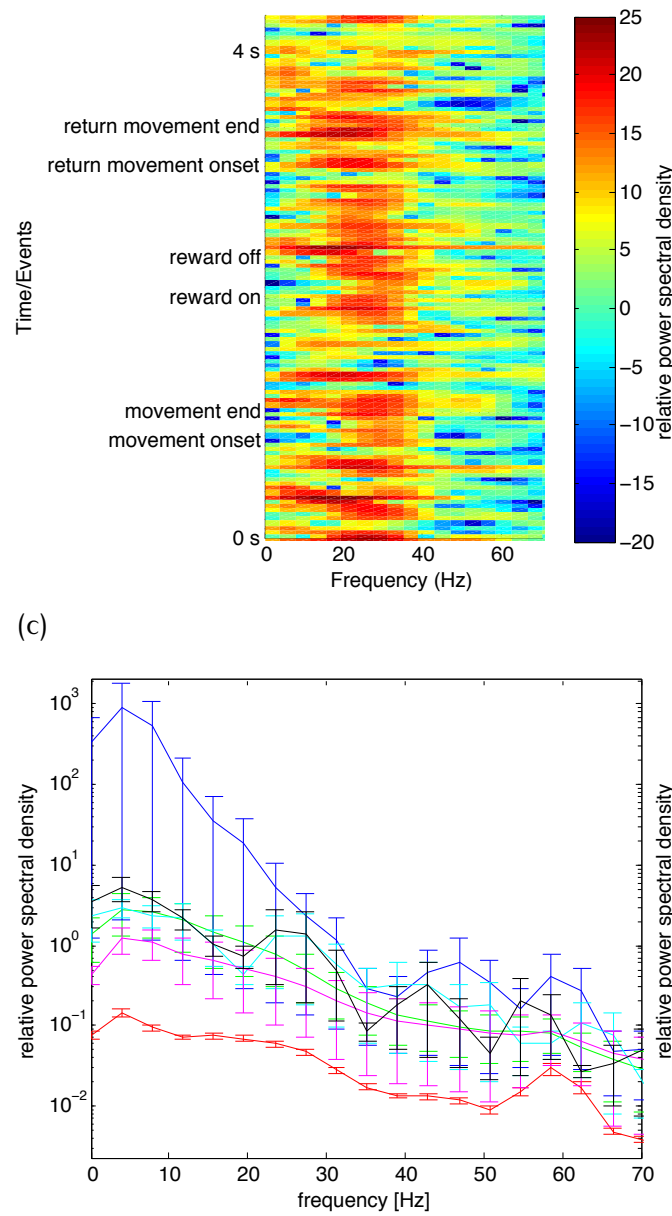

(b)

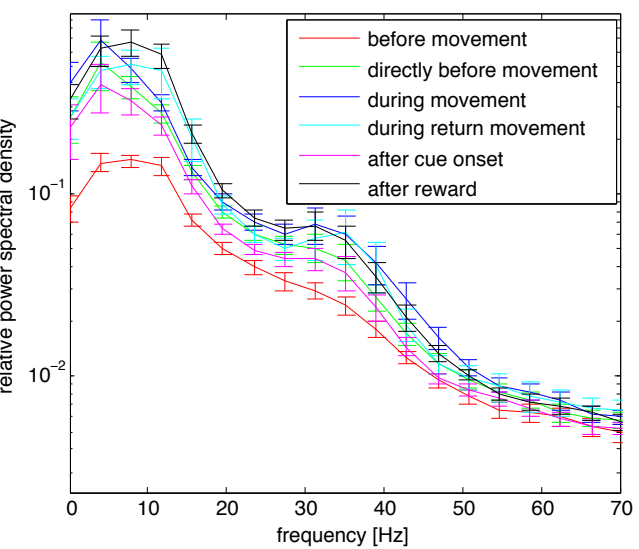

(d)

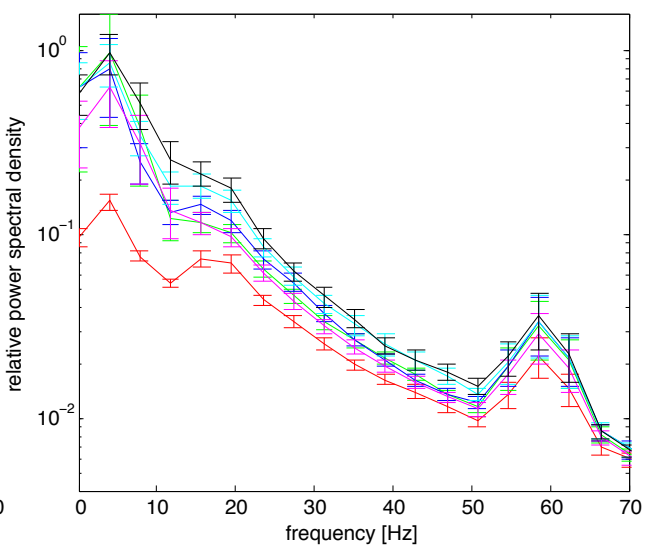

Figure 5.3: (a) Example of a C.Pi LFP spectrogram during one trial in one recording. Beta bursts occur both before and during movement. Averaged LFP spectra of (b) M1, (c) $C_{1} P i$ and (d) VLa, using time windows described in the legend of (b). Only LFP data from movable electrodes were used, and all spectra were normalized with the total power of the spectrum "before movement onset". After normalization, the LFP spectra were first averaged over all trials of one recording, and then over all recordings. Error bars indicate the standard errors of the mean (SEM) with respect to averaging over recordings, i.e. after averaging over trials. The little insets highlight the spectrum "during movement". Spectra vary highly between recordings, seen as large error bars, but also between the time windows. 
(e)

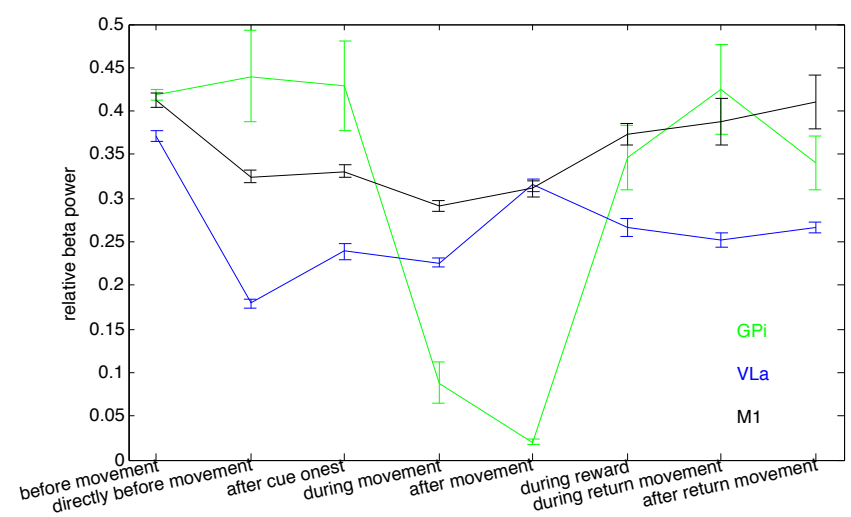

Figure 5.3: (Continued) (e) When looking at the beta power $(12-30 \mathrm{~Hz})$, normalized with the total power of each spectrum, variations during movement can be seen.

\subsubsection{Low noise correlations between C.Pi and VLa}

Noise correlations, or spike count correlations, are frequently used to quantify synchrony between spike trains when the very precise spike timing is of minor importance [Perkel et al., 1967]. Here, we use noise correlations to estimate whether spike counts in $\mathrm{G}_{1} \mathrm{Pi}$ and VLa were correlated, for example due to movement relateddischarge which is passed from G.Pi to VLa.

Both positive and negative spike noise correlations within M1, GPi and VLa were common (Fig. 5.4). However, noise correlations between C.Pi and VLa, as expected for the transmission of movement-related discharge, were only slightly stronger than the control noise correlations (Fig. 5.4). Introducing a time lag between C.Pi and VLa did not lead to strong correlations, as shown for some example time lags in Fig. 5.4. Nevertheless, we could see slight variations in the average noise correlations and the average absolute value of the noise correlations when varying the time lag (Fig. 5.5).

In particular, the average noise correlations were maximal for a time lag of $-68 \mathrm{~ms}$ and minimal for a time lag of $+66 \mathrm{~ms}$ (Fig. 5.5(a)). The positive peak at negative time lags indicates that there is a positive correlation between C.Pi activity and VLa activity when $\mathrm{C}_{1} \mathrm{Pi}$ activity was shifted back in time. Such a time lag of VLa behind $\mathrm{C}_{1} \mathrm{Pi}$ would be expected for transmission of activity from C.Pi to VLa, although at smaller lags. The positive correlation could for example be achieved by rebound-inhbition or disinhibition. In contrast, at positive time lags, an inverse correlation between $\mathrm{C}_{1} \mathrm{Pi}$ and VLa activity could be seen which indicates that G Pi firing was on average 
suppressed after the occurrence of VLa spikes.

Notably, as visible in Fig. 5.4(right), G.Pi-VLa noise correlations were about equally positive and negative and therefore partly canceled out each other during averaging, leading to near-zero values for the average correlations. The average absolute correlations (Fig. 5.5(b)), in contrast, were significant at any time lag between $-500 \mathrm{~ms}$ and $500 \mathrm{~ms}$, but maximal at at $-33 \mathrm{~ms}$ and $+3 \mathrm{~ms}$. Interestingly, both average correlations and average absolute correlations showed rhythmic modulations at high beta frequencies (Fig. 5.5, small insets).

In conclusion, we found only very low noise correlations between C.Pi and VLa. This stands in contrast to the common assumption that discharge in C.Pi, for example accompanying movement, suppresses firing in VLa. When averaging noise correlations of all neuron pairs, we found a slight rhythmic modulation at high beta frequency with the time delay between $C_{1} P i$ and VLa. This could be a first indication that the common influence of many neuron is important for transmission of information between $\mathrm{C}_{1} \mathrm{Pi}$ and VLa.

\subsubsection{Both GPi and VLa showed movement-related discharge}

Since noise correlations (Section 5.3.2) were low, we quantified movement related discharge in $\mathrm{C}_{1} \mathrm{Pi}$ and VLa independently. For example, a lack of movement-related discharge in VLa could explain the absence of noise correlations. In line with Zimnik and Turner [2015], we found both increasing and decreasing firing rates at movement onset (see Fig. 5.6 for examples). Fig. 5.7 depicts spike-density averages around movement onset after classification.

A small fraction of cells showed discharge that was both increasing and decreasing around movement onset, and some cells did not show any significant modulation of discharge. With outward movement onset, many cells in both C.Pi and VLa increased their firing rate $\left(46 \%\right.$ in $\mathrm{C}_{1} \mathrm{Pi}$ and $58 \%$ in VLa, not significantly different in $\chi^{2}$-test, $\left.p>0.05\right)$, whereas only few cells decreased their firing rate $\left(12 \%\right.$ in $\mathrm{C}_{1} \mathrm{Pi}$ and $19 \%$ in VLa, not significantly different in $\chi^{2}$-test, $\left.p>0.05\right)$. Assuming that GiPi would gate VLa activity via disinhibition, we would expect that increases in G.Pi firing rate lead to reductions in VLa firing rate and vice versa. Since this was not the case in our data, we could not confirm such gating so far. 

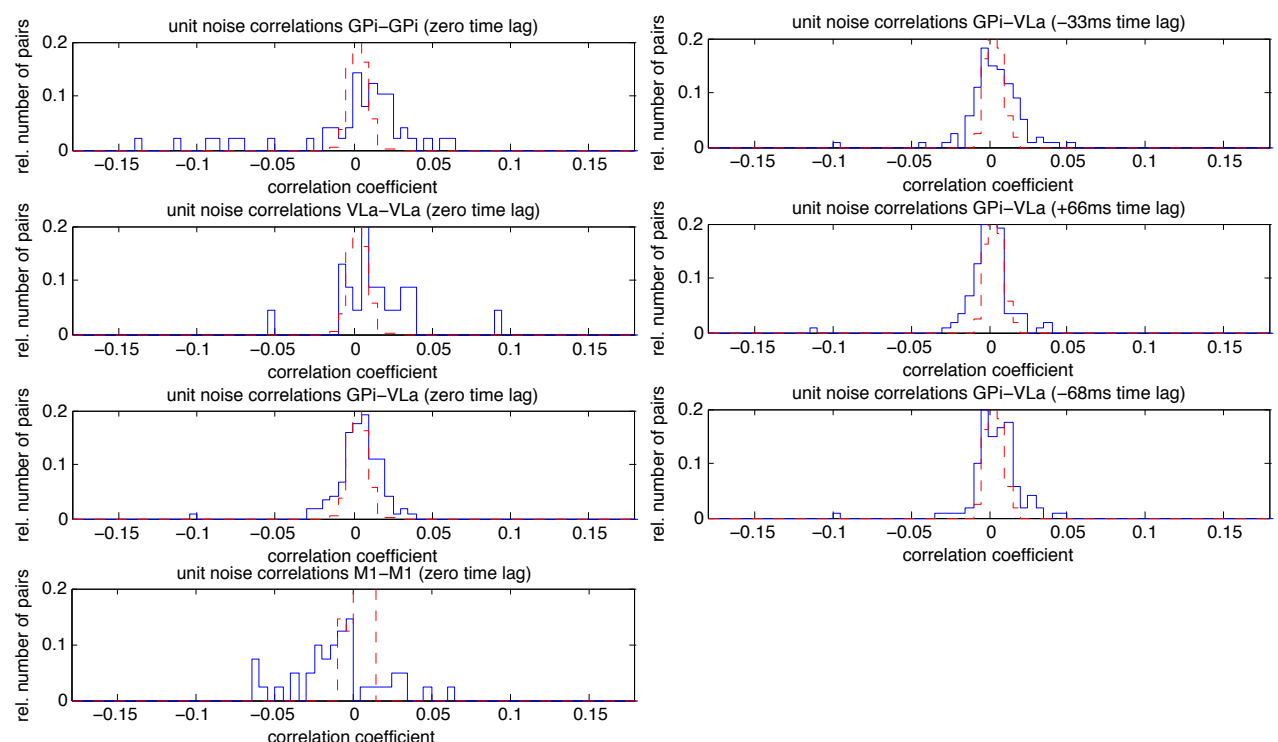

Figure 5.4: Noise correlation histograms for $10 \mathrm{~ms}$ time bins. We show the number of pairs (y-axis) that have a certain correlation coefficient (x-axis). Control distributions, based on shuffled controls, are drawn with red dashed lines. While significant noise correlations were common within $\mathrm{C}_{1} \mathrm{Pi}$, VLa and $\mathrm{M} 1$, only few cell pairs with significant noise correlations existed between G.Pi and VLa. At some example time lags (right), both positive and negative significant correlations were still present. $A$ positive time lag indicates that VLa leads GPi.

However, the situation changed for return movement. High variations in the baseline could be seen (Fig. 5.7(c,d)), referring to the holding period and impeding the detection of changes in discharge. Nevertheless, we could also detect movementrelated discharge for return movement, showing both increases (25\%) and decreases $(34 \%)$ in $\mathrm{CPi}_{1}$, and equally many increases and decreases in VLa (both 19\%). In particular, $\mathrm{C}_{1} \mathrm{Pi}$ cells decreased their firing rates more often during return movement than during outward movement ( $p<0.001, \chi^{2}$-test). Return movement was initiated intrinsically. It is therefore possible that some part of the discharge during outward movement is related to the visual cue rather than to movement itself. For both outward and return movement, G.Pi as well as VLa showed discharge up to around $200 \mathrm{~ms}$ prior to movement initiation.

To sum up, movement-related discharge was present in both C.Pi and VLa. Hence, the absence of strong noise correlations cannot be explained by missing discharge. 
(a)

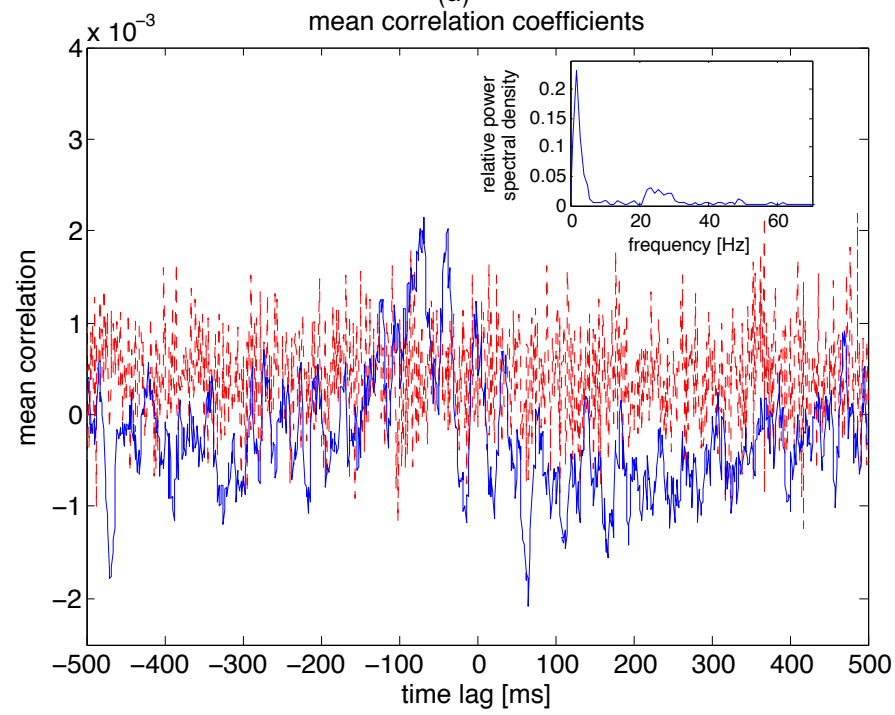

(b)

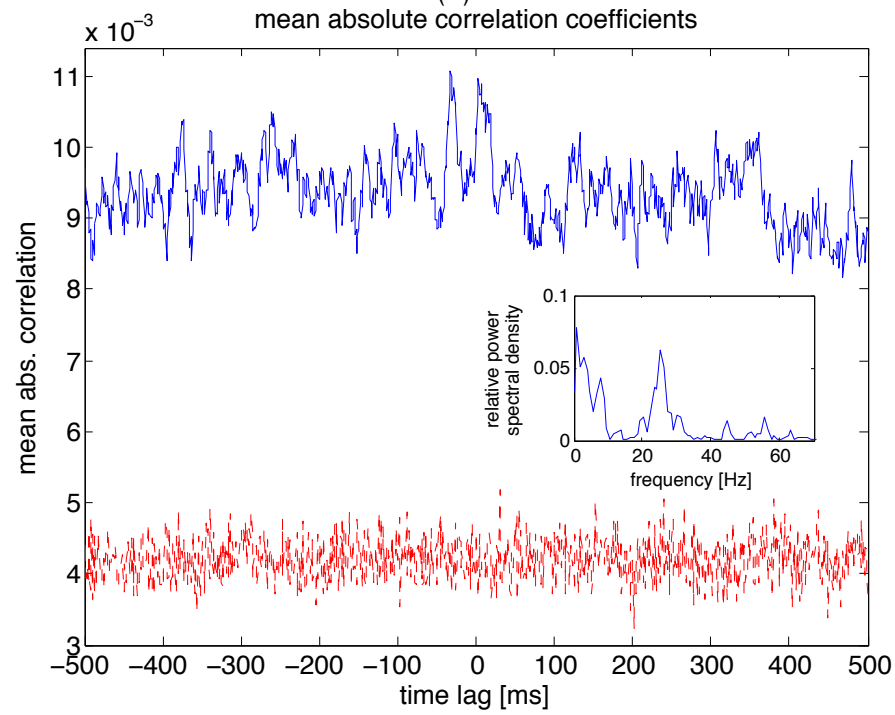

Figure 5.5: (a) Average noise correlation coefficients between GPi and VLa dependent on the time lag, using $10 \mathrm{~ms}$ time bins. The average correlation is maximal at time lag $-68 \mathrm{~ms}$ and minimal at time lag $+66 \mathrm{~ms}$. (b) Average absolute noise correlation coefficients. The highest absolute correlations are seen at $-33 \mathrm{~ms}$ and $+3 \mathrm{~ms}$. Red dashed lines indicate control distributions. For both figures, a positive time lag means that VLa leads C.Pi. The small insets show the power spectra of the blue curves. Next to a peak at low frequencies $(<5 \mathrm{~Hz})$, they also both show a peak at high beta frequencies $(20-30 \mathrm{~Hz})$. 


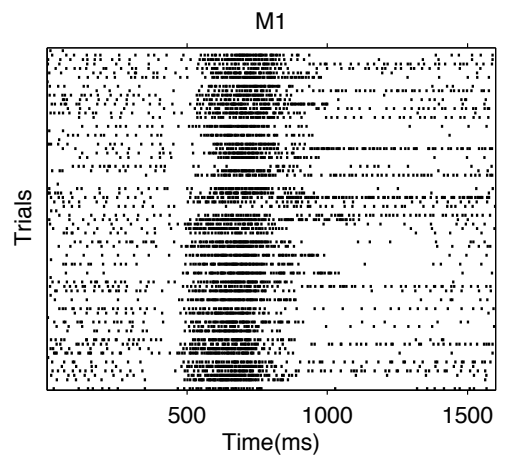

VLA

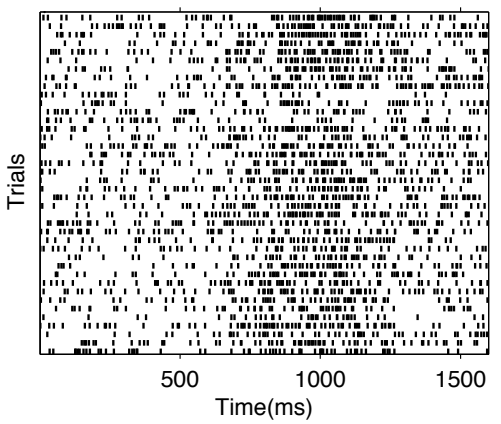

GPI

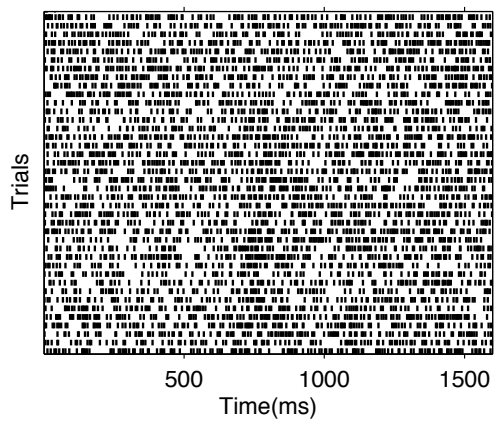

M1
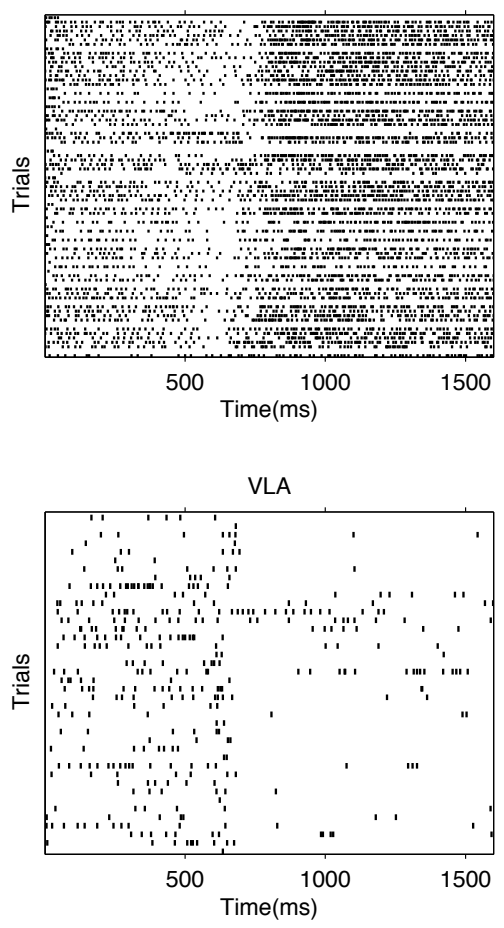

GPI

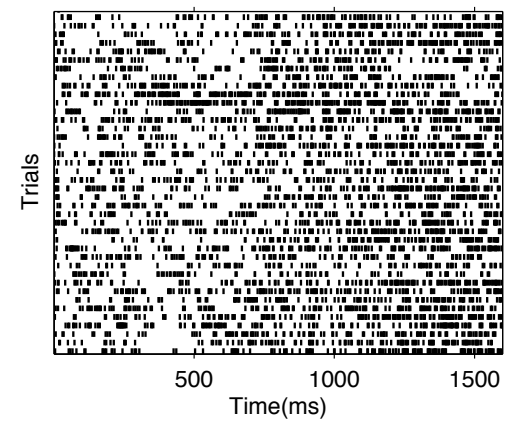

Figure 5.6: Raster plots of M1, G.Pi and VLa spiking activity in an example recording, aligned with outward movement onset. Movement starts at time $800 \mathrm{~ms}$. Increases, decreases and constant firing rates can be seen in all nuclei. 
Our results thus suggest that discharge in VLa is primarily not directly caused by discharge in $\mathrm{C}_{1} \mathrm{Pi}$, but rather indirectly or by common input.

\subsubsection{Spike correlations between C.Pi and VLa were absent or weak and not modulated during movement}

Next to noise correlations, we also looked at precise spike synchrony quantified by CCFs. This approach also allowed to detect the timing of possible correlations via the CCF time lag. Spike correlations were weak in all M1, C.Pi, VLa and between $\mathrm{C} . \mathrm{Pi}$ and VLa (examples shown in Fig. 5.8(a-d)). Although the CCF peak histograms in M1 were significantly different from the control distribution (Fig. 5.8(e), $p<0.05$ for all time windows except "directly before movement" and "during return movement", two-sample Kolmogorov-Smirnov (K-S) test), we could not detect any significant increase during outward or return movement which has been described in literature [Riehle et al., 1997, Engel and Singer, 2001] ( $p>0.1$, two-sample K-S test).

In contrast, no significant spike correlations were found within $\mathrm{C}_{1} \mathrm{Pi}$, also not during movement (Fig. 5.8(f), $p>0.1$, two-sample K-S test). This finding has been reported in Bergman et al. [1998] for pallidal cells in general, and in Morris et al. [2003] for the substantia nigra pars reticulata ( $\mathrm{SNr}$ ). VLa correlations were significant for all time windows except "during movement" (Fig. 5.8(g), $p<0.05$, two-sample K-S test). Finally, spike correlations between $C_{1} P i$ and VLa were very weak, and significant for only $11.7 \%$ of all pairs. Still, the C.Pi-VLa peak CCF distributions were significant for all time windows except "directly before movement" (Fig. 5.8(h), $p<0.01$, two-sample K-S test). We did not detect a significant change with movement compared to rest ( $p>0.05$, two-sample K-S test).

Thus, not only noise correlations between C.Pi and VLa were weak, but also spike CCFs. Direct or indirect interactions between single $\mathrm{C}_{1} \mathrm{Pi}$ and VLa cells therefore were not as prominent as expected for gating or even driving of thalamus by basal ganglia output. 
(a)

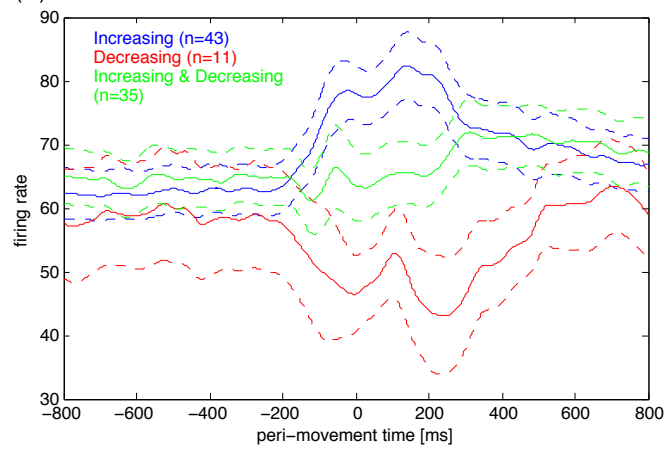

(c)

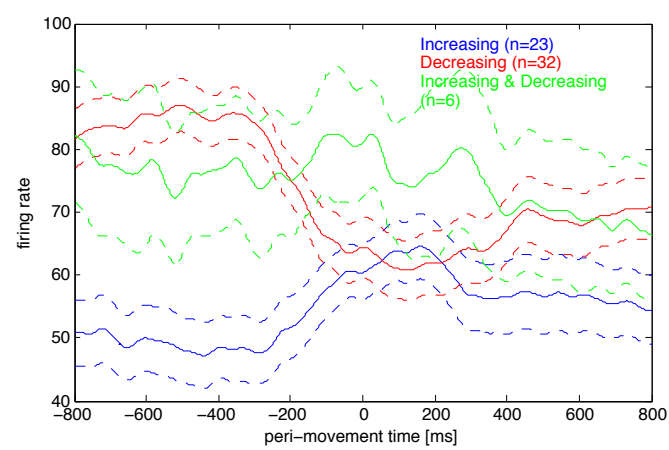

(e)

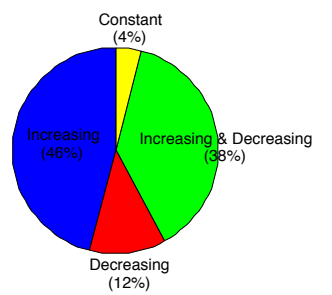

(f)

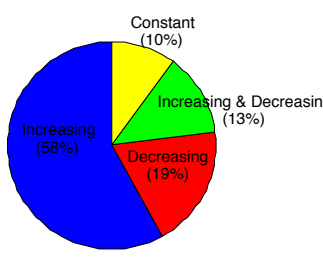

(b)

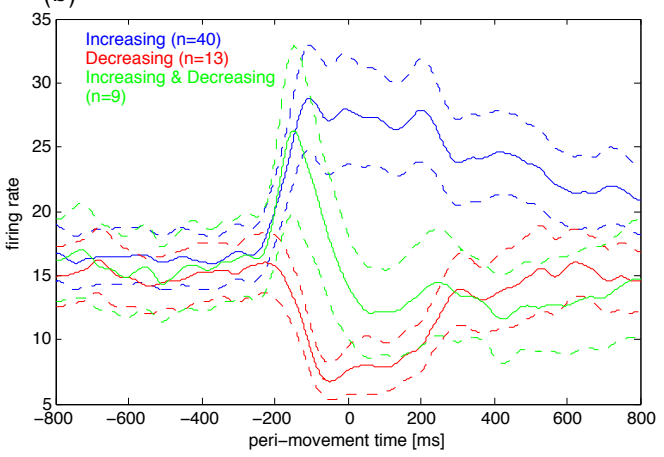

(d)

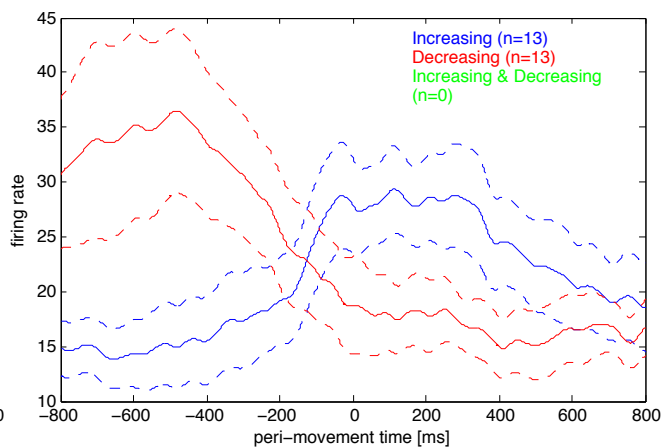

(g)

(h)

Figure 5.7: Spike-density averages around movement onset. Dashed lines indicate the SEM. During outward movement, both in GPi (a) and VLa (b), increases in firing rate were most common. However, increases and decreases in firing rates were roughly equally common during return movement in C.Pi (c) and VLa (d). As return movement was preceded by the holding period, the baseline showed high variations in this case. $n$ denotes the number of cells in certain classification. The relative fractions of cells in each class are shown in panels $(e-h)$. (e,f) refer to C.Pi and VLa, respectively, at outward movement, and $(g, h)$ to $C_{1} P i$ and VLa, respectively, at return movement. 
(a)

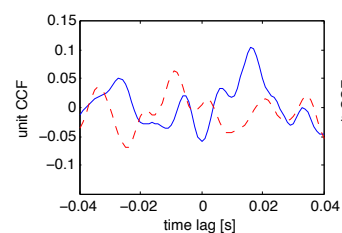

(b)

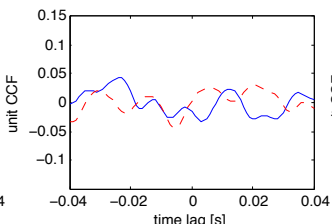

(c)

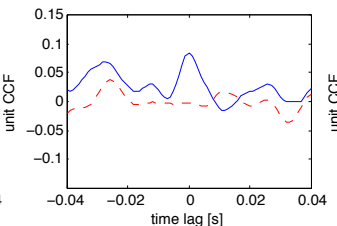

(d)

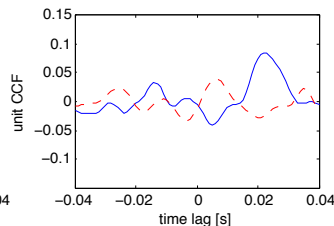

(e)
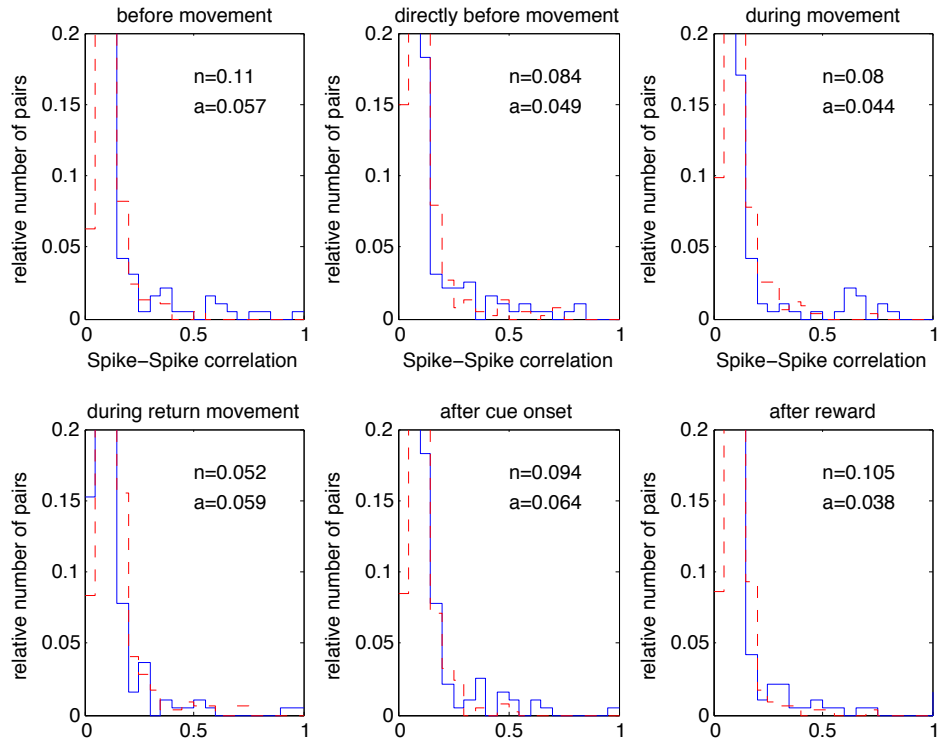

(f)
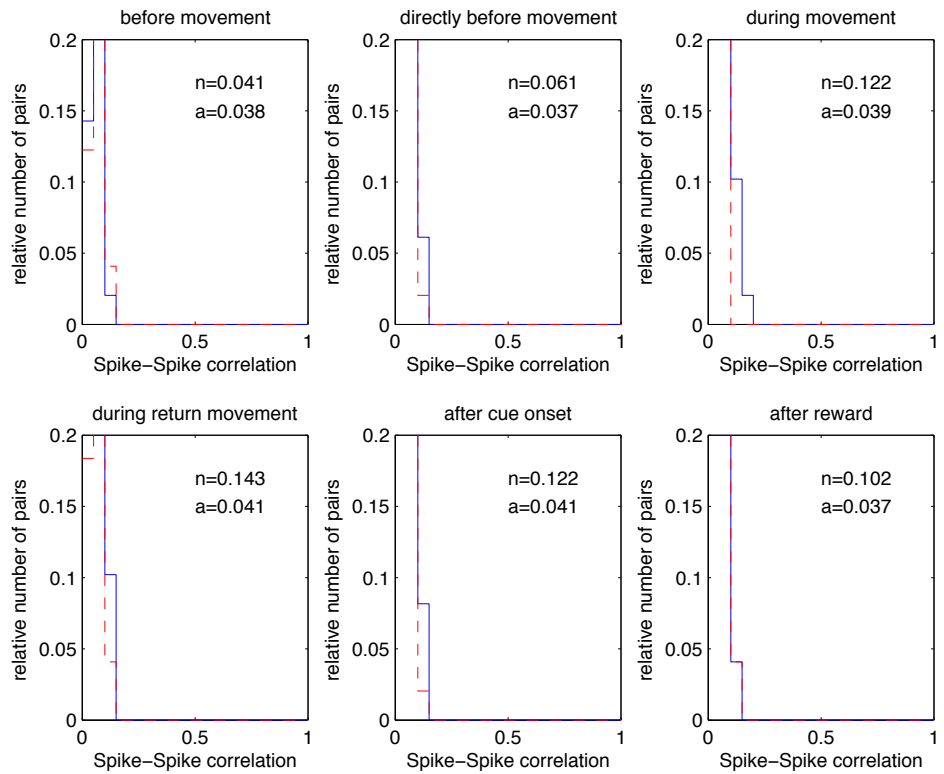

Figure 5.8 
(g)
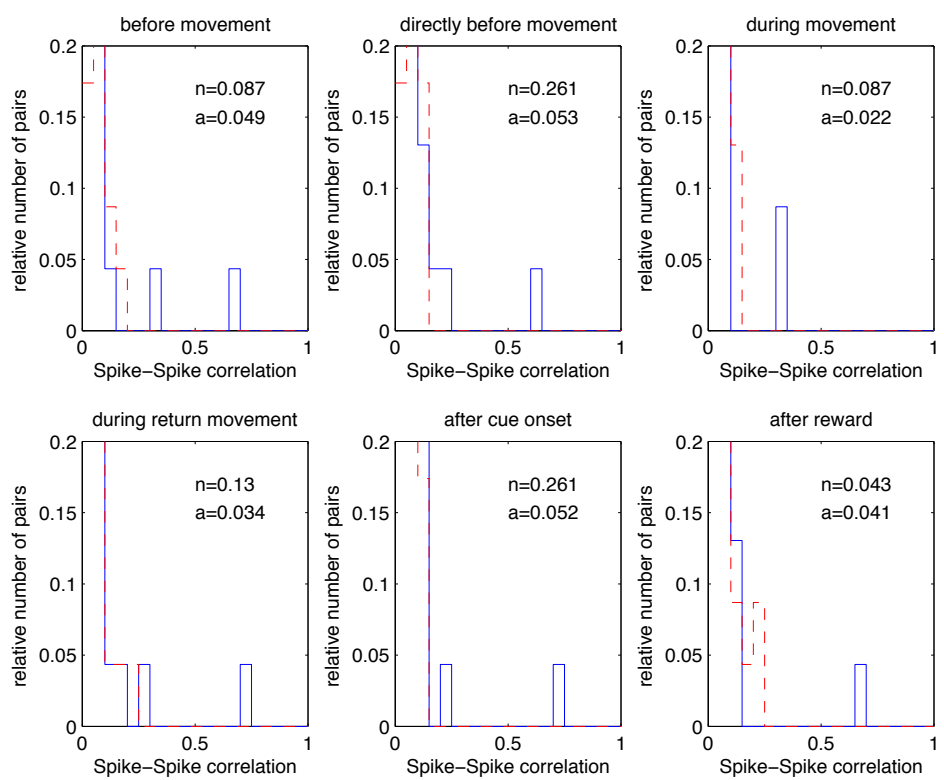

(h)
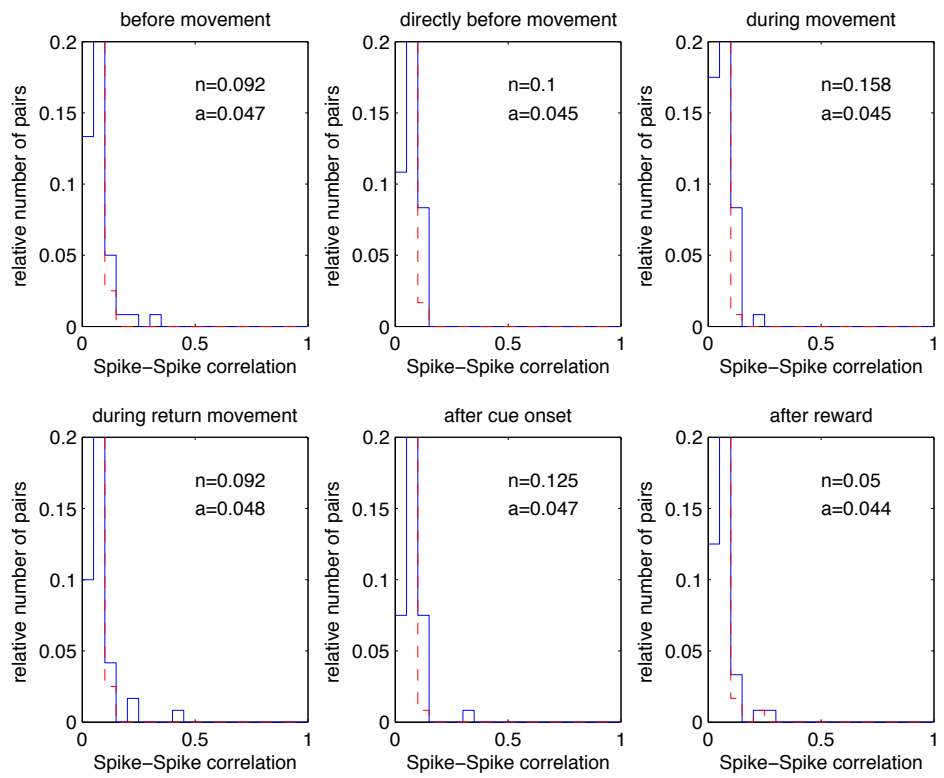

Figure 5.8: (Continued) Example unit CCFs (averaged over all trials of one recording) in M1 (a), G.Pi (b), VLa (c) and between C.Pi and VLa (d) are shown in blue, and their respective shuffled controls red dashed. Histograms of peak spike CCF values of all recordings: (e) in $\mathrm{M1}$, ( $\mathrm{f}$ ) in $\mathrm{C}_{1} \mathrm{Pi},(\mathrm{g})$ in VLa, (h) between $\mathrm{C}_{1} \mathrm{Pi}$ and VLa. Red dashed marks indicate control distributions. $n$ denotes the fraction of significant pairs $(95 \%$ confidence), and $a$ the average peak correlation. Spike correlations were very weak, in particular in C.Pi (f). 


\subsubsection{LFP correlations between CPi and VLa were strong, and could only scarcely directly be explained by cortical input}

LFPs integrate the signals of many neurons, and might thus give an indication on population dynamics. As for spikes, we also calculated LFP CCFs to detect synchrony and its timing. In contrast to spike correlations, LFP correlations were strong and occurred at various time lags.

Fig. 5.9 shows example LFP CCFs between CPPi and VLa (a), between C.Pi and M1 (b) as well as between VLa and M1 (c), all having higher amplitudes than their respective shuffled controls. Notably, all LFP correlations between C.Pi-VLa pairs were significant, and all histograms were significant compared to the control distribution (Fig. 5.9(d), $p<0.05$, two-sample K-S test), but also between M1 and $\mathrm{C}_{1} \mathrm{Pi}$ (Fig. 5.9(f)). M1-VLa correlations were significant for most pairs (Fig. 5.9(h)). Remarkably, VLa often led M1 (Fig. 5.9(i)), but M1 often led G.Pi (Fig. 5.9(g)). Nevertheless, correlations between $\mathrm{C}_{1} \mathrm{Pi}$ and VLa occurred mainly around time lag zero (Fig. 5.9(e)).

We saw a large difference between LFP and spike CCFs: LFP correlations were much stronger. Although it is not clear what properties exactly are reflected in the recorded LFPS, this could be another indication that the activity of many cells is necessary to transmit information.

Both C.Pi and VLa might be modulated directly or indirectly by M1. To estimate what influence $\mathrm{M} 1$ had on LFP correlations between $\mathrm{CP}$ i and VLa, we predicted these correlations by using the method of partial spectra [Halliday and Rosenberg, 1999], with the M1 LFP from implanted electrodes as the source signal. The pCCFs were rather flat, although often significant (Fig. 5.10(a), histograms differed from control distribution, $p<0.05$, two-sample K-S test). Corrected LFP correlation peak values are shown in Fig. 5.10(b). The cCCFs were thus still strong and most correlations stayed significant, indicating that the majority of LFP correlations between C.Pi and VLa were not or only partly predicted by M1.

\subsubsection{Spike-LFP correlations could not confirm a clear feed-forward structure of the GPi-VLa connection}

If LFPs in VLa are caused by synaptic currents, and these synaptic currents arise from spiking in $\mathrm{C}_{1} \mathrm{Pi}$, we would expect to see a correlation between $\mathrm{C}_{1} \mathrm{Pi}$ spikes and VLa LFPs. In contrast, correlations between VLa spikes and C.Pi LFPs should be 
(a)
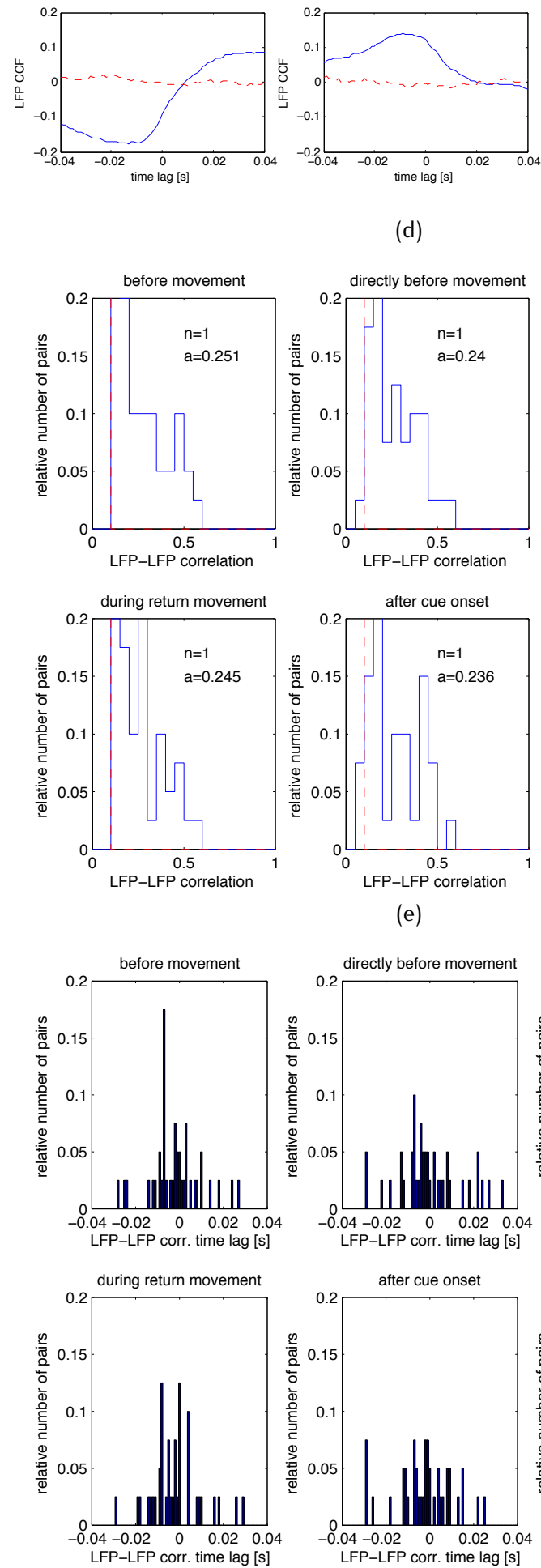

(d)

(b)

(c)
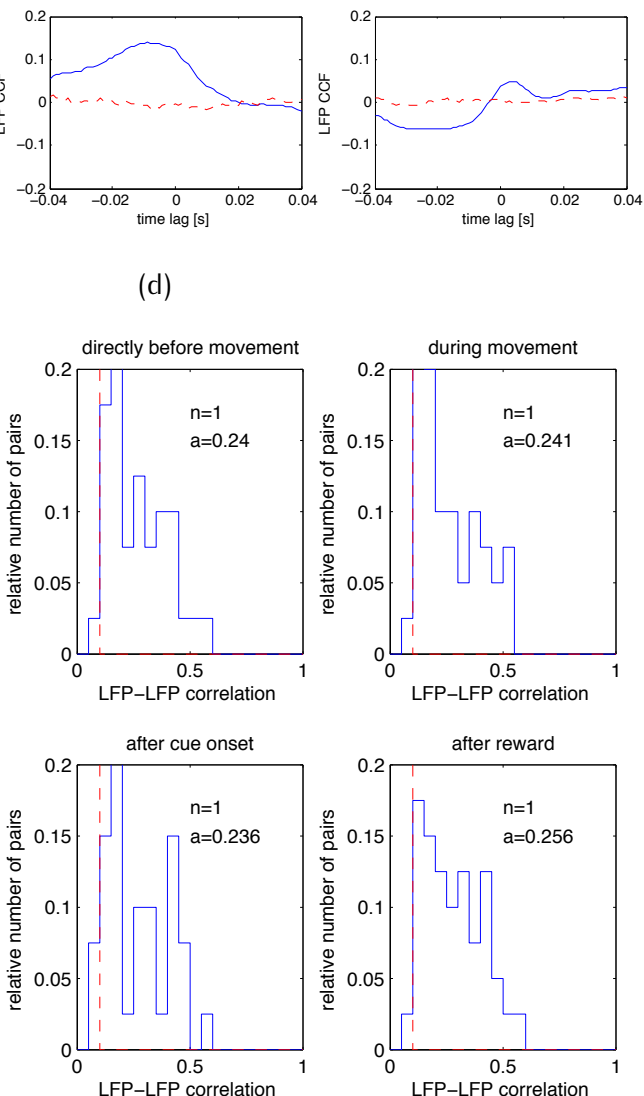

(e)

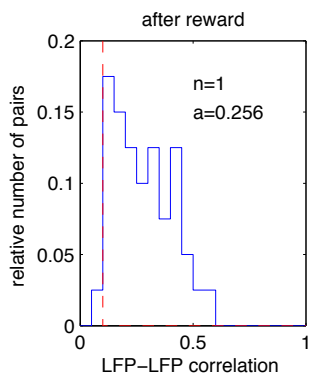

LFP-LFP correlation
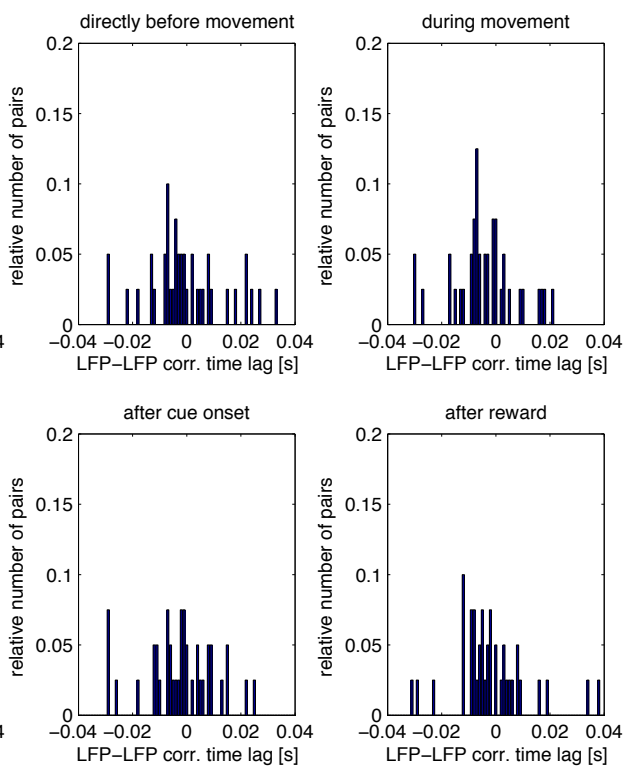

Figure 5.9 
(f)
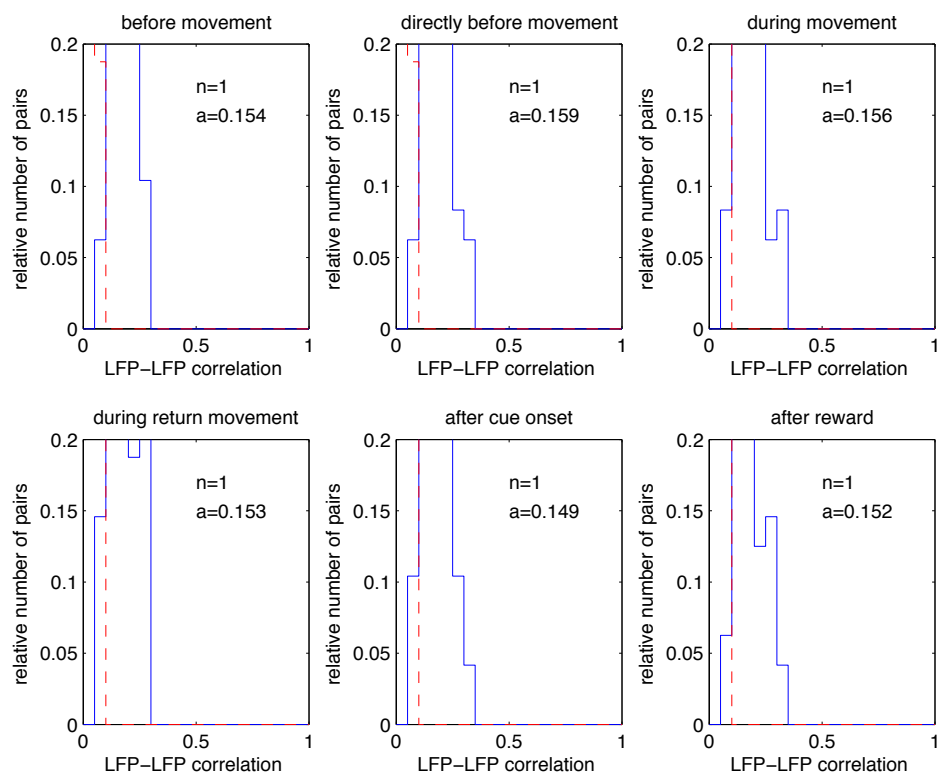

(g)
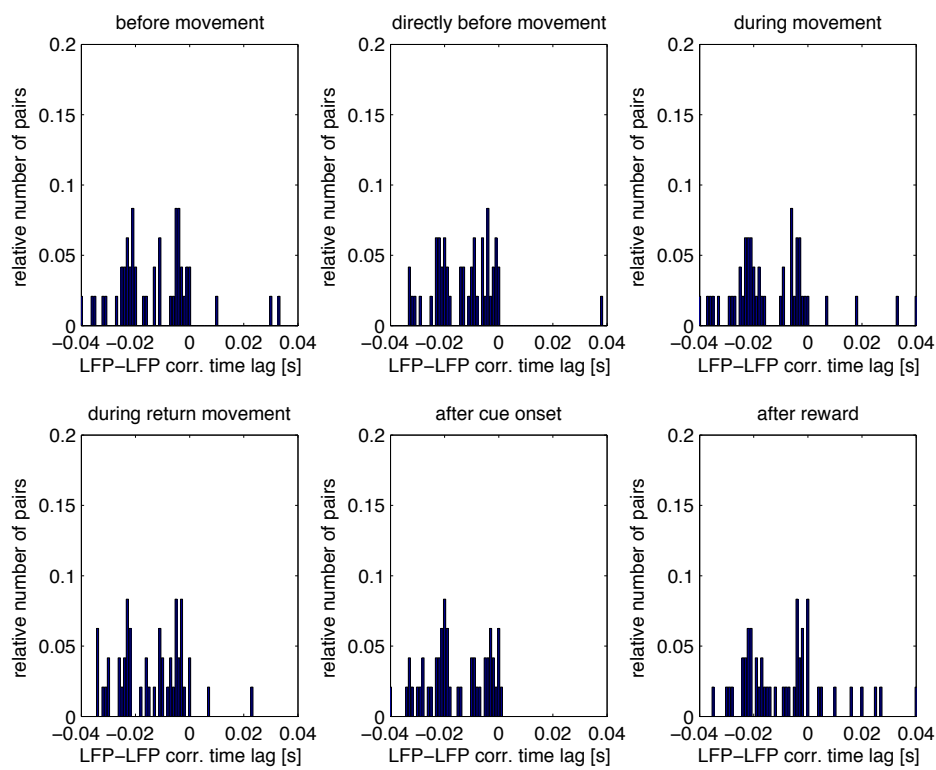

Figure 5.9: (Continued) (a-c) Examples of LFP CCFs, between CPi and VLa (a), between $\mathrm{M} 1$ and $\mathrm{C}_{1} \mathrm{Pi}$ (b) and between M1 and VLa (c). Red dashed lines indicate shuffled controls. Histograms of peak C.Pi LFP - VLa LFP CCF values (d) and location of significant highest peaks (e). Positive time lags indicate a lead of VLa. 
(h)
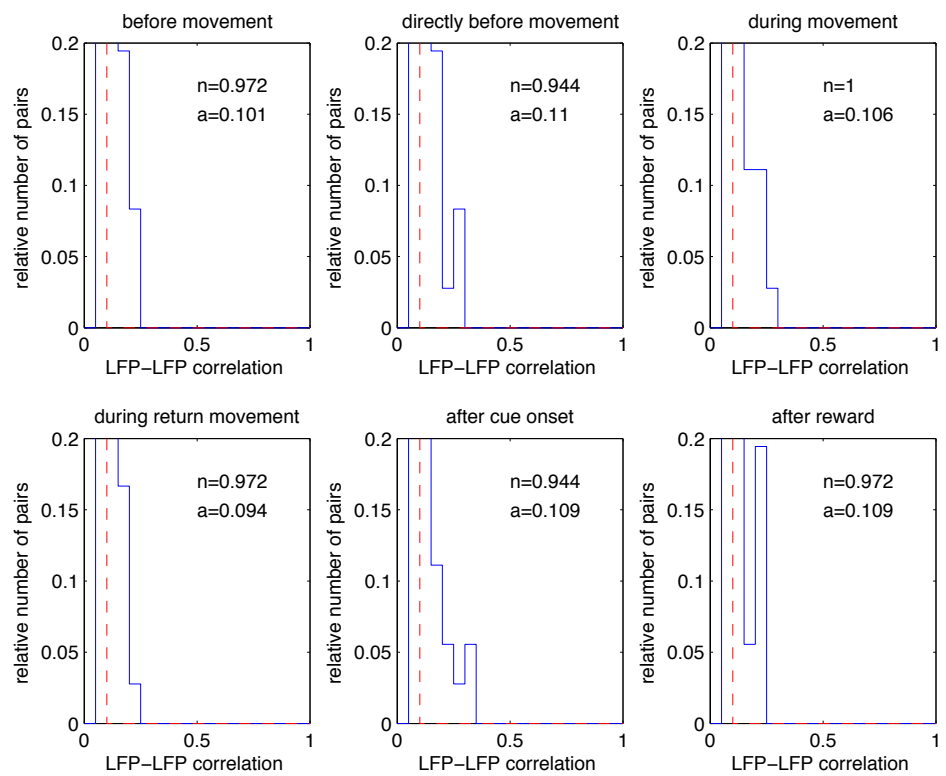

(i)
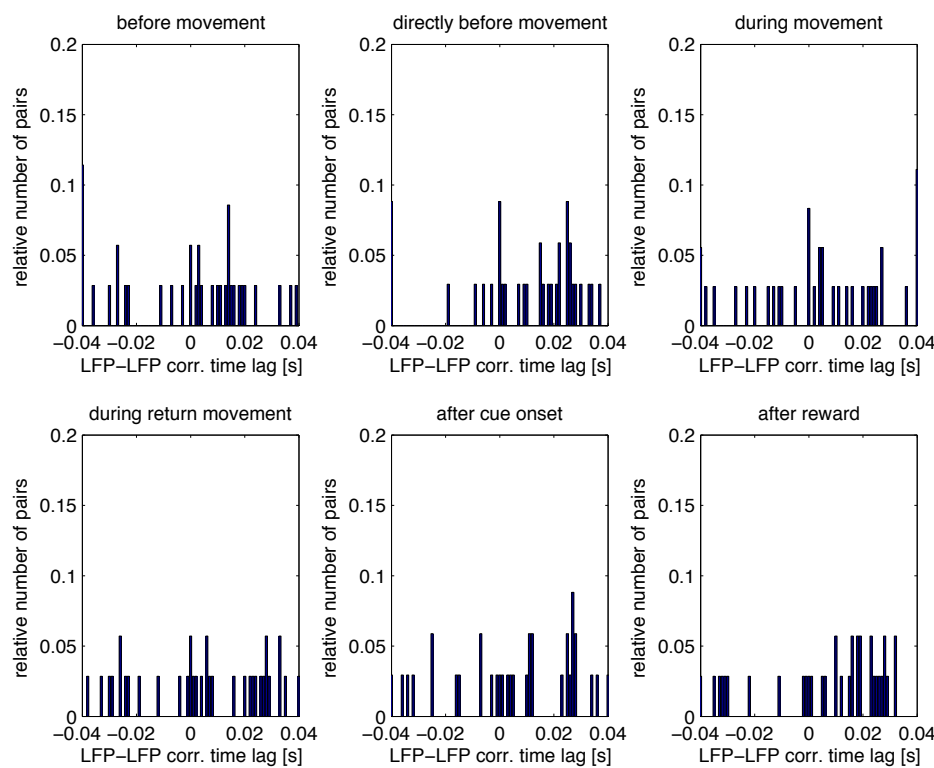

Figure 5.9: (Continued) $(f, g)$ Same for M1-C,Pi CCFs, where a positive time lag indicates a lead of $\mathrm{GPi}$. (h,i) Same for M1-VLa CCFs, where a positive time lag indicates a lead of VLa. Red dashed marks depict control distributions. n denotes the fraction of significant pairs (95\% confidence), and $a$ the average peak correlation. LFP correlations were strong, especially also between C.Pi and VLa (d). 
(a)
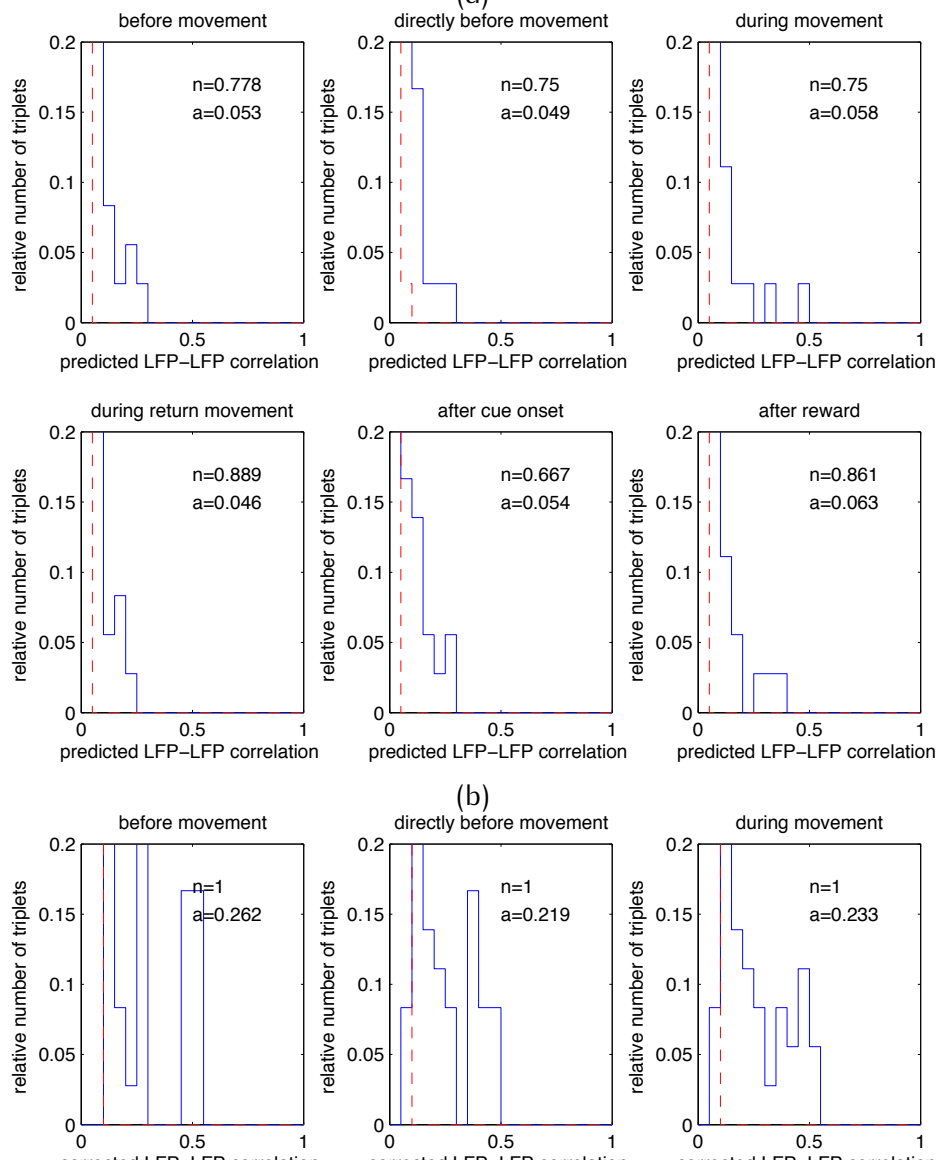

(b)
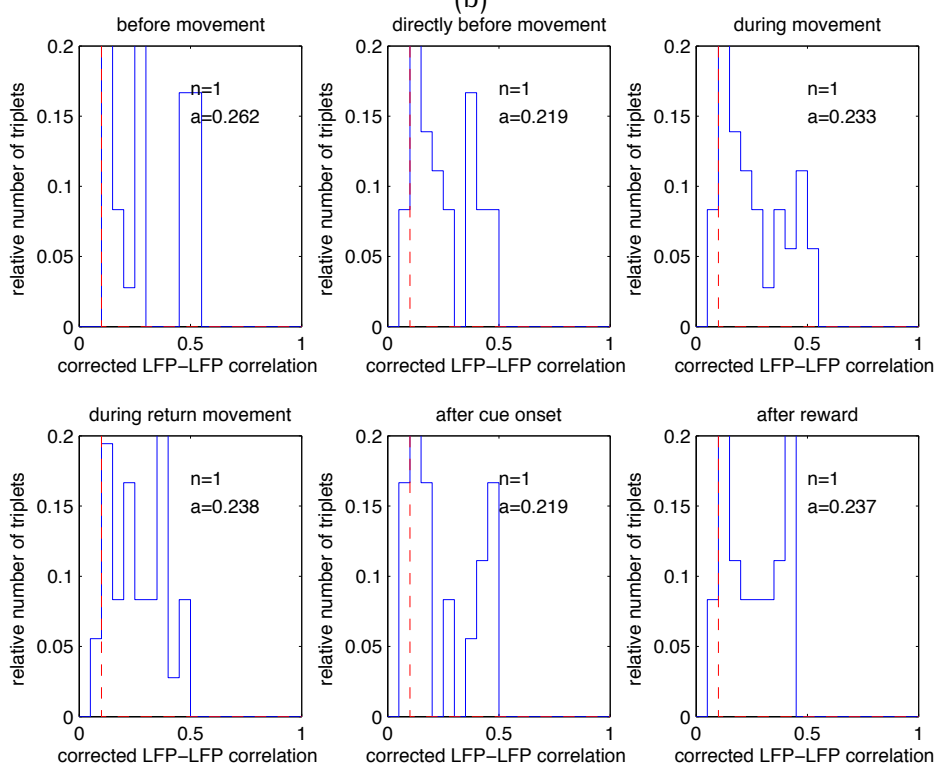

Figure 5.10: (a) Histograms of M1-predicted G.Pi-VLa LFP CCF (pCCF) peaks. (b) Histrograms of peaks of CPi-VLa LFP CCFs (cCCFs), corrected by predicted CCFs shown in (a). Red dashed marks indicate control distributions. $n$ denotes the fraction of significant pairs (95\% confidence), and $a$ the average peak correlation. Only few LFP correlations between GPi and VLa were predicted by M1. 
lower, as VLa does not, as far as we know, project directly back to GiPi. We computed spike-LFP correlations in this section to test those hypotheses, but found few pairs with significant spike-LFP coupling.

Even in M1 (example shown in Fig. 5.11(a)), the correlation coefficient histogram was only different to the control histogram for the outward movement period (Fig. 5.11(c), $p<0.05$, two-sample K-S test). Spike-triggered LFP averages in GPi were not different from the control (Fig. 5.11(d), $p>0.05$, two-sample K-S test), and low compared to spike-triggered averages in $\mathrm{M} 1$ both at rest and during movement ( $p<0.001$, two-sample K-S test). We were in particular interested whether C.Pi spikes evoked currents in VLa and therefore correlated with VLa LFPs (example Fig. 5.11(b)). As depicted in Fig. 5.11(c) and (f), these correlations were both weak, especially for C.Pi spikes and VLa LFPs. C.Pi spike - VLa LFP correlations were significantly weaker than VLa spike - C.Pi LFP correlations both during and before outward movement ( $p<0.05$, two-sample K-S test). We could therefore not confirm an increased processing of $\mathrm{C}_{1} \mathrm{Pi}$ spike information to VLa compared to the other direction with this analysis. Also correlations between M1 LFPs and C.Pi spikes were significant only during return movement (Fig. 5.11(g), $p<0.05$, two-sample K-S test). Stronger correlations were detected between M1 LFPs and VLa spikes (Fig. 5.11(h), significantly different from control distribution before movement, during return movement and after cue onset, $p<0.05$, two-sample K-S test).

Hereafter, we could not show a clear difference between correlations of GPi spikes and VLa LFPs, and the reversed situation. However, spike-LFP correlations in general were very low, even in M1. Many spike-LFP pairs might be necessary to see significant effects. One important factor could also be that the spectra of our LFP signals cover many frequencies, potentially restricting spike-LFP coupling [Martin and Schröder, 2012].

Spike-triggered averages are a common and sensitive measure to detect correlations between spikes and LFPs [Fries, 2005]. However, in some situations the results can be false negative, for example when spike timing is related only to the absolute value of the LFP. Although this is not what we expect, we did a second analysis here, showing the average spike counts in all LFP phases. To test if C.Pi spikes occurred preferably at a certain VLa LFP phase, we plotted the average number of spikes over the LFP phase in Fig. 5.12(a).

LFP traces were beforehand filtered in eight equidistant frequency bands between 5 and $45 \mathrm{~Hz}$. Only few LFP phases had spike counts above the 95\% confidence level, 
(a)
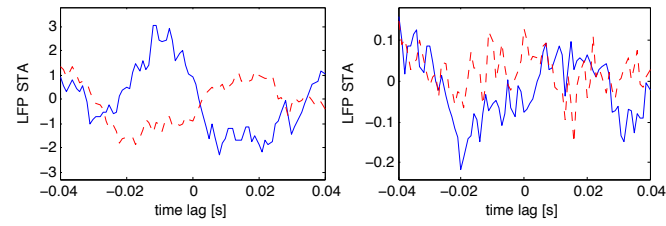

(c)
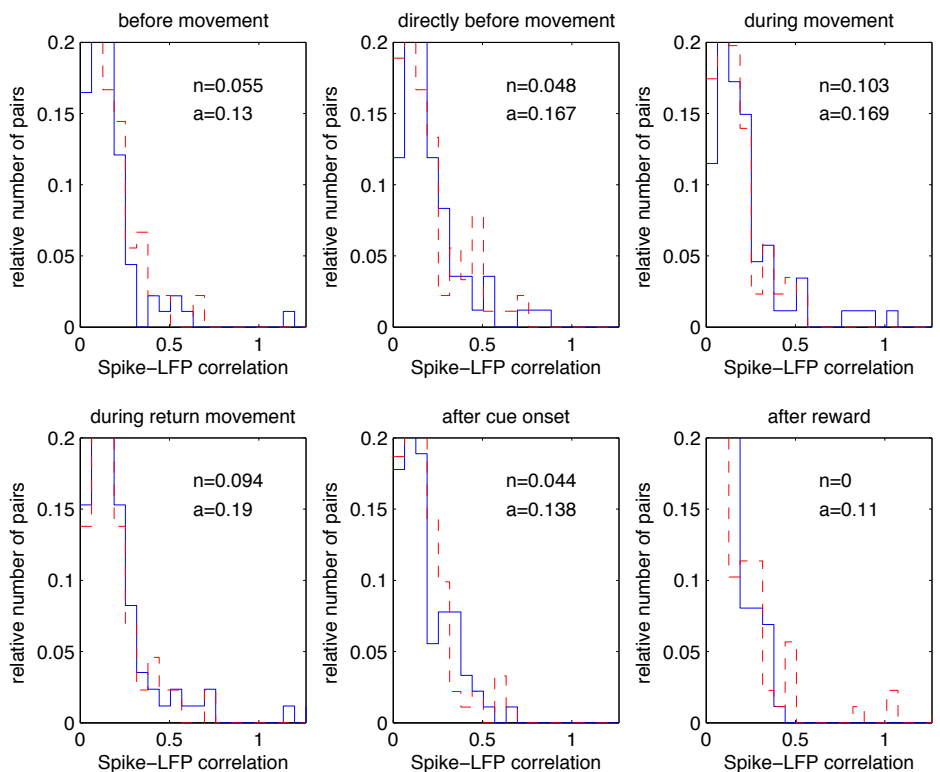

(d)
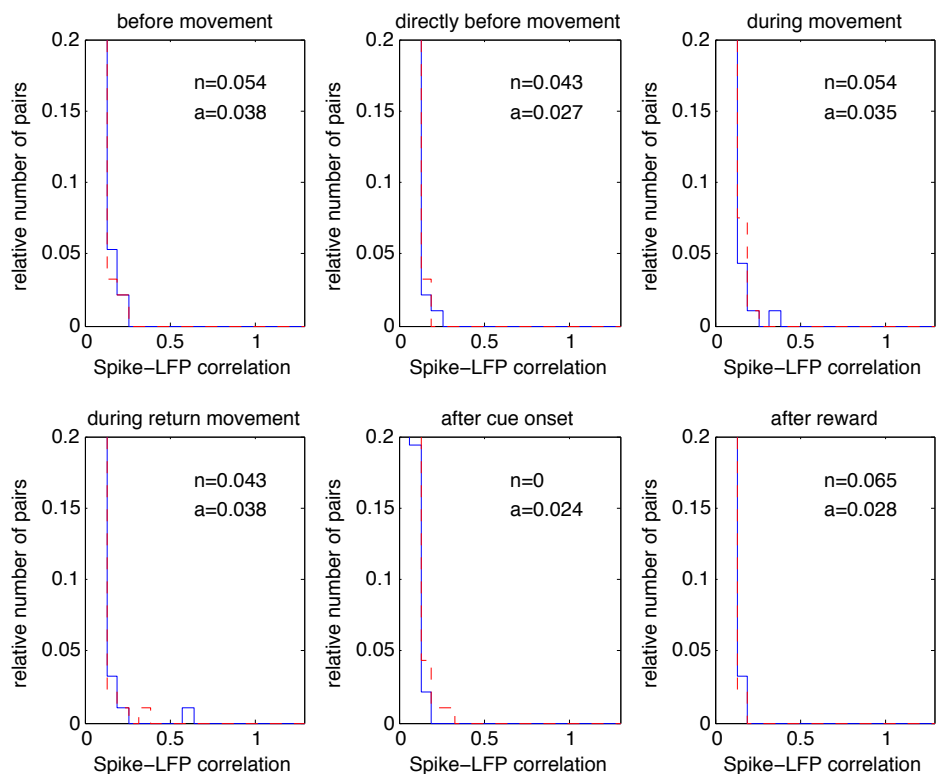

Figure 5.11 
(e)
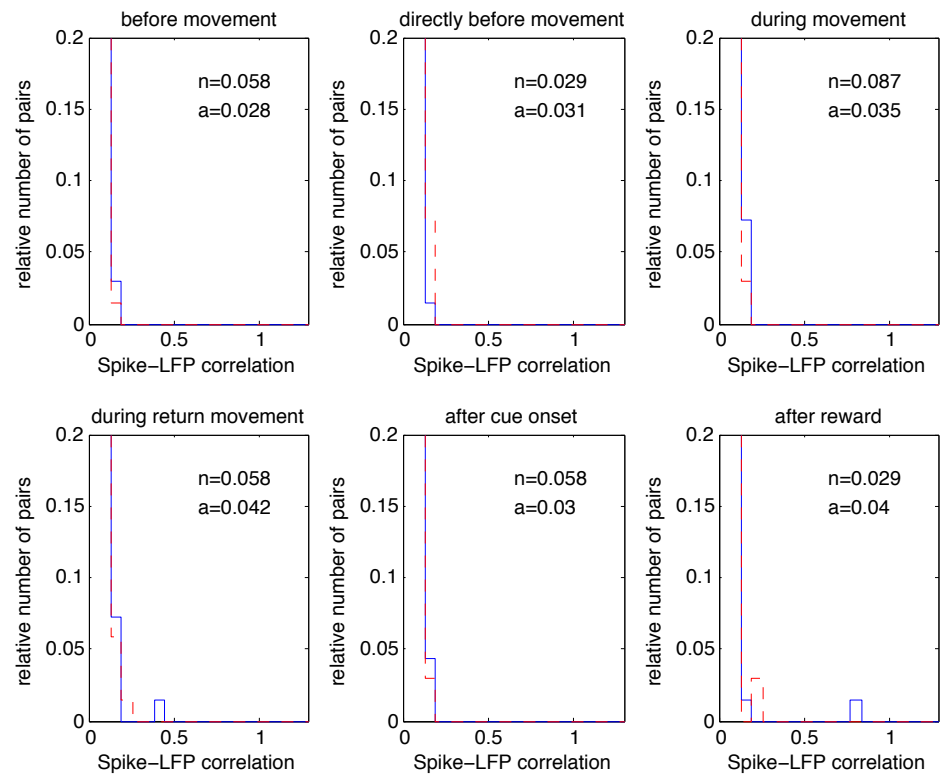

(f)
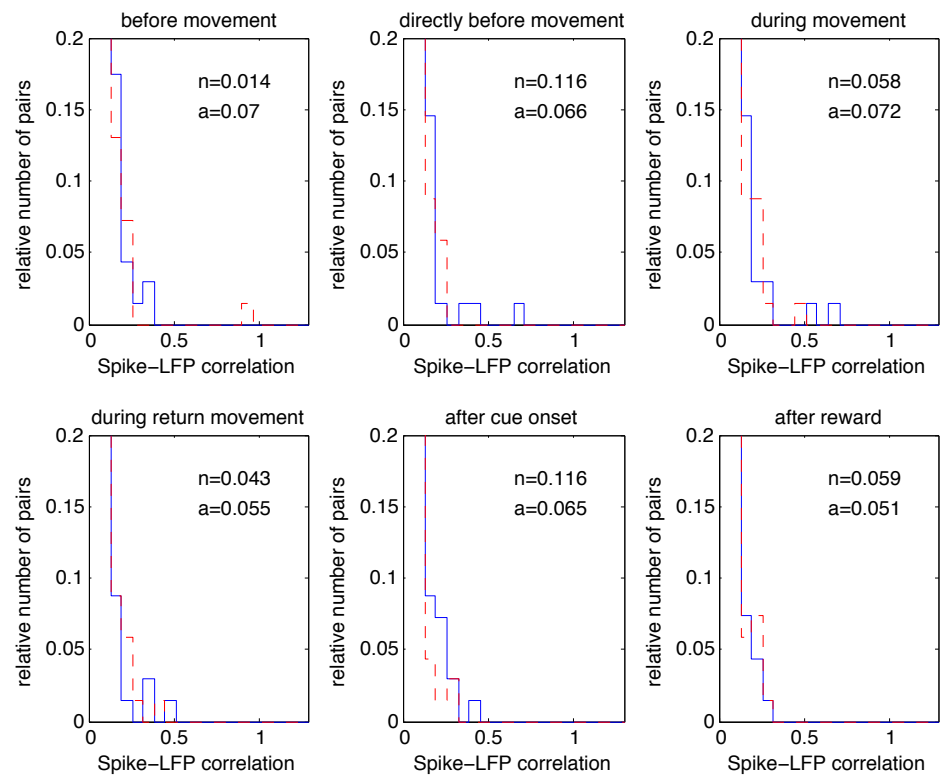

Figure 5.11: (Continued) (a) An example LFP STA using M1 spikes and M1 LFPs. (b) Same for GiPi spikes and VLa LFP. 
(g)
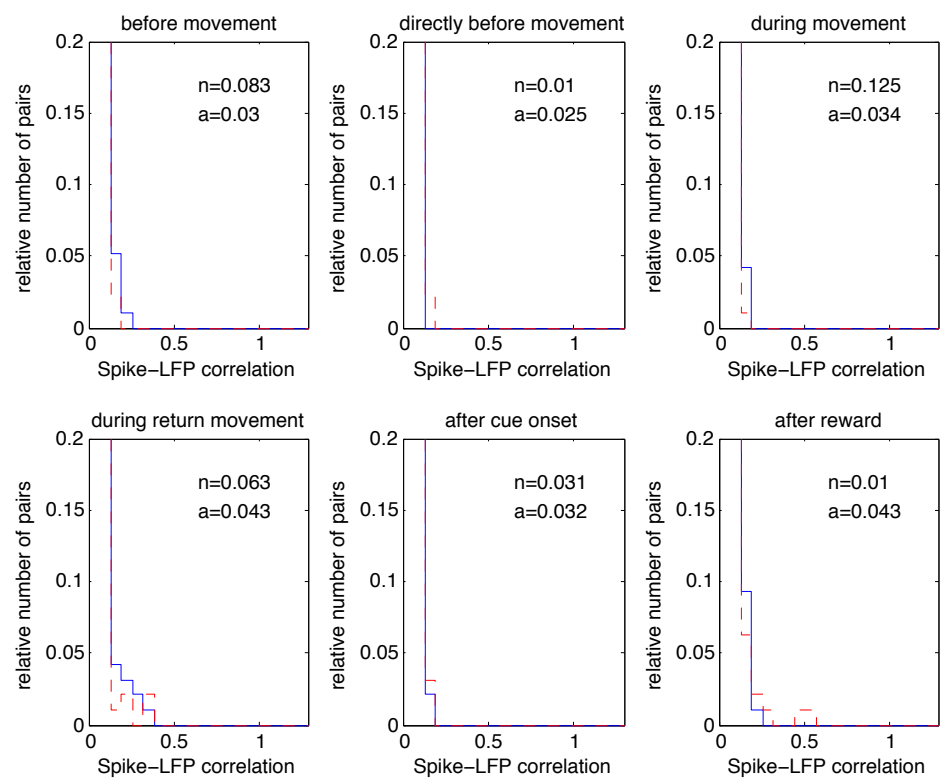

(h)
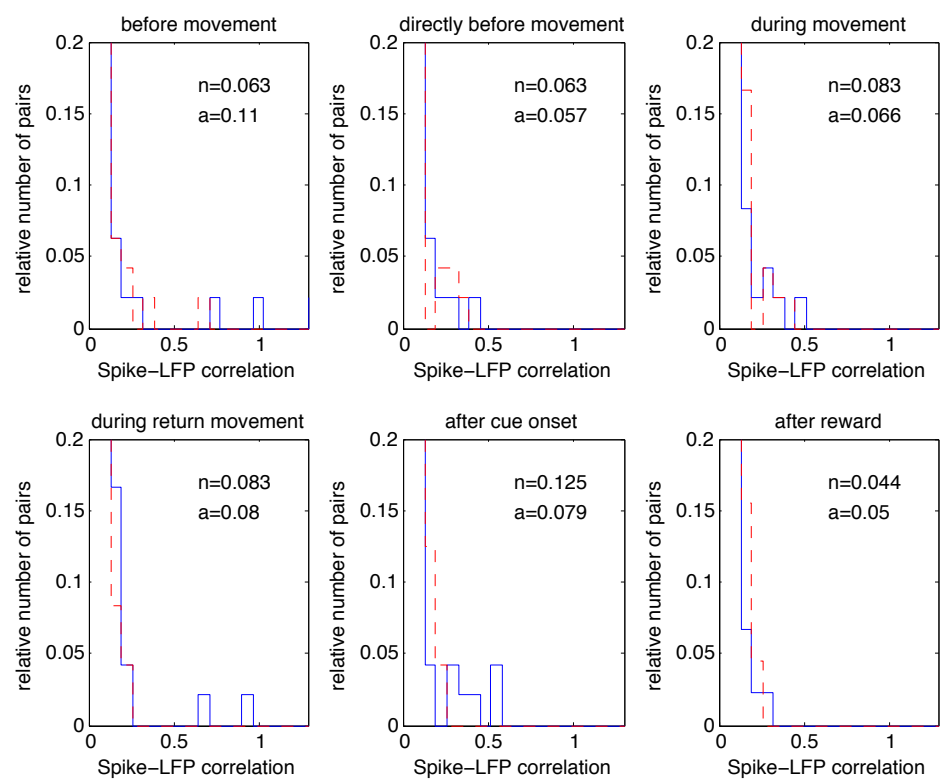

Figure 5.11: (Continued) Histograms of spike-LFP correlation coefficients within (c) M1, (d) C.Pi, (e) between G.Pi spikes and VLa LFP, (f) between VLa spikes and $C_{1} P i$ LFP, (g) between C.Pi spikes and M1 LFP, (h) between VLa spikes and M1 LFP. n denotes the fraction of significant pairs (95\% confidence), and $a$ the average peak correlation. Spike-LFP correlations were weak in all regions, including M1. 
and none of them reached the $99.9 \%$ confidence level (not shown). A very similar picture could be seen for the other direction: VLa spikes did not occur more frequently at certain C.Pi LFP phases than at others (Fig. 5.12(b)). Hence, the latter analysis confirmed very the low spike-LFP correlations, which we already quantified by spiketriggered LFPs.

\subsection{Discussion}

We investigated the dynamics of the monkey basal ganglia-thalamic connection during movement, using both LFP and single unit recordings. The most important results are summarized in Table 5.3. LFP traces in C.Pi and VLa showed brief bursts of high beta activity, and the average occurrence of these bursts was modulated around movement as it has been described in detail for striatum and motor cortex [Feingold et al., 2015]. Compared to spiking in M1, basal ganglia output spikes were uncorrelated, both at rest and during movement. We did not find a direct entrainment of VLa spikes or LFPs to C.Pi spiking, notably also not during movement, although movement-related discharge was common in both GPi and VLa. In contrast, LFP correlations between $\mathrm{C}_{1} \mathrm{Pi}$ and VLa were high and significant for all pairs. Even after subtracting a linear influence of M1, these LFP correlations stayed strong. Our results indicate that, compared to cortical information transmission, the connection between basal ganglia and thalamus is weak on a cell-to-cell basis. Still, when combining noise correlations of all $C_{1} P i-V L a$ cell pairs, we found slight but significant modulations in the mean correlation dependent on the time lag. A rhythmic modulation of the average noise correlations at high beta frequency could be seen. Since we did not find large changes in pairs of cells with high noise correlations, this is most likely an effect of slightly different noise correlations in a large number of cell pairs. In particular, we found excitatory influences of GiPi on VLa, contradictory to the assumption that the main influence of $\mathrm{GPi}_{\mathrm{P}}$ on VLa is inhibitory, and slight inhibitory influences of VLa on C.Pi.

It is not clear how exactly basal ganglia information is relayed to thalamus, including which thalamic neurons receive basal ganglia input. Both thalamic principal neurons and interneurons are targeted by C.Pi fibers [Ilinsky et al., 1997, KultasIlinsky et al., 1997]. If the main influence of GPi on VLa is via interneurons, principal neurons in VLa could be indirectly excited by GPi via disinhibition of interneurons. Moreover, VLa projections diffusively targeting medium spiny neurons in striatum have been described [McFarland and Haber, 2000, Smith et al., 2004]. Via striatum, VLa neurons could thus have an inhibitory effect on C.Pi neurons. We sketch the 
(a)
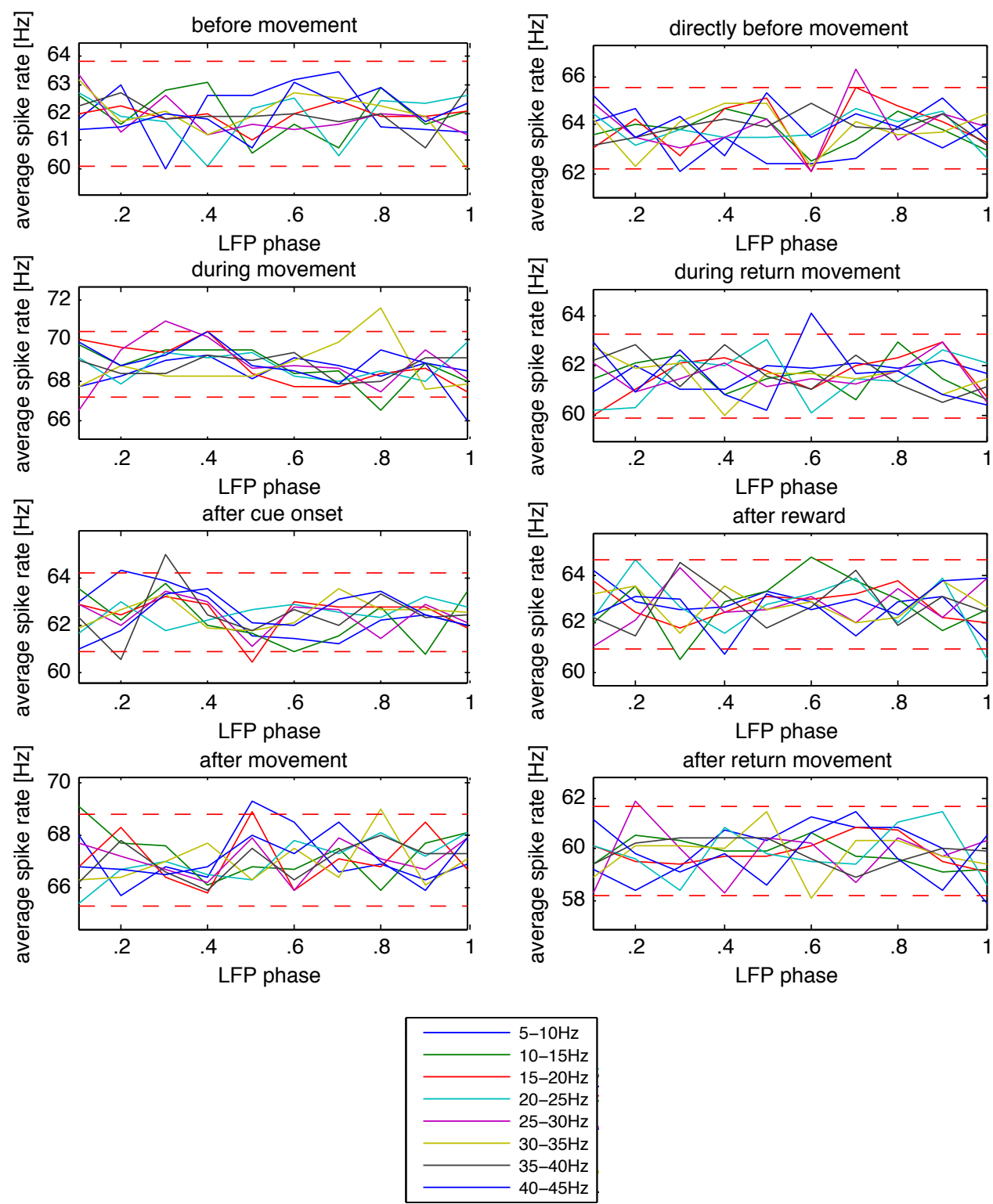

Figure 5.12: Do spikes preferably occur at a certain LFP phase? Average C.Pi spike counts are shown for different VLa LFP phases (a). 
(b)
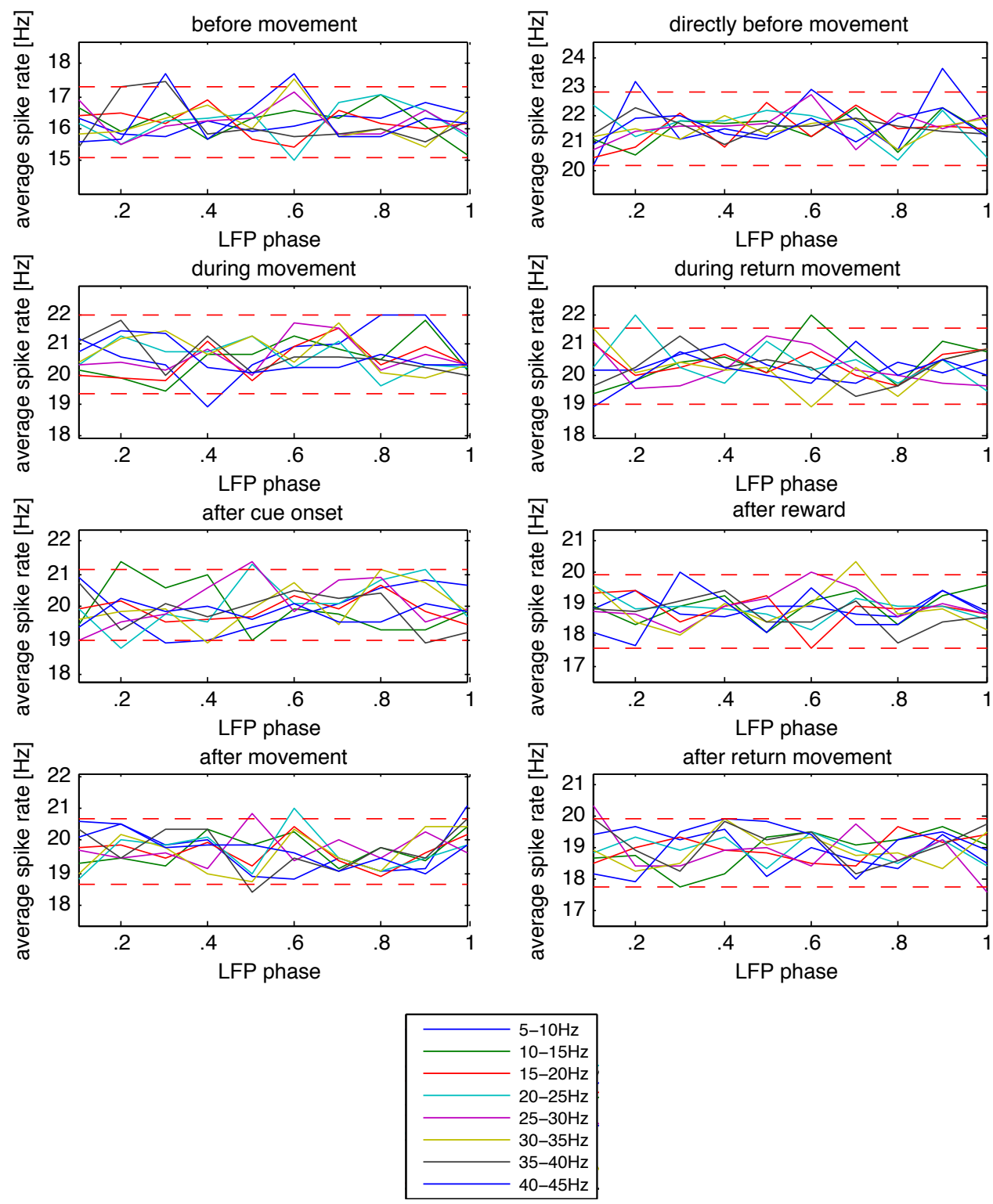

Figure 5.12: (Continued) Average VLa spike counts for different G.Pi LFP phases (b). Red dashed marks indicate 95\% confidence intervals. No clear accumulation of spikes at a certain LFP phase was found, which confirmed weak or nonexistent spike-LFP coupling. 
architecture of the resulting loop between basal ganglia and thalamus in Fig. 5.13. Although the connection between VLa and striatum might be sparse, it cannot be excluded that a feedback loop between $\mathrm{C}_{1} \mathrm{Pi}$, VLa and striatum exists. In conclusion, the connection between basal ganglia and thalamus is likely more complex than suspected based on recordings in birds, and might extend beyond the sole role of a driver or gater. LFP dynamics of this connection are not necessarily informative on cell-to-cell information flow between GPi and VLa. However, movement-related information might be transmitted via large numbers of neurons from the basal ganglia to thalamus, as reflected in coherent LFP signals.

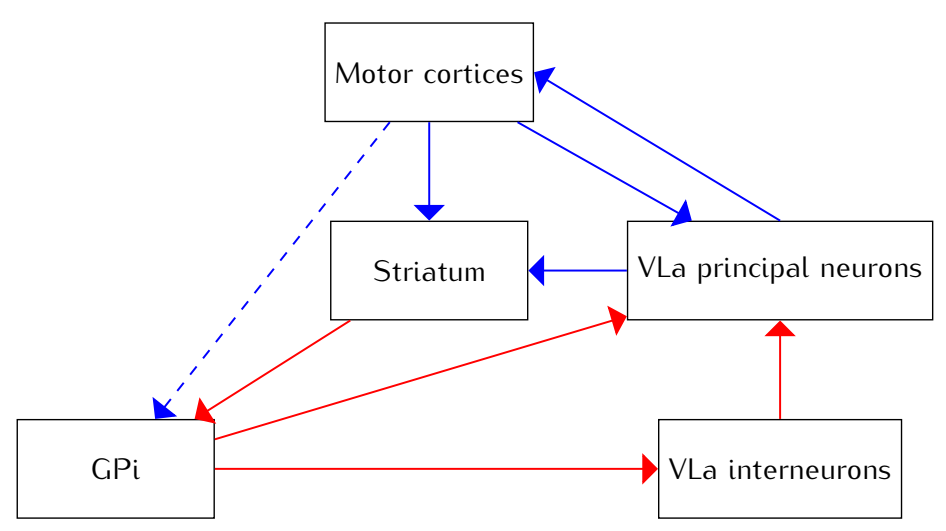

Figure 5.13: Architecture of connections between basal ganglia and thalamus. Inhibitory pathways are drawn red, excitatory pathways in blue. Pathways with sparse evidence are shown dashed. The influence of C.Pi neurons on principal VLa neurons could be indirect, involving thalamic interneurons. Furthermore, feedback from VLa to $\mathrm{C}_{1} \mathrm{Pi}$ could be possible via striatum.

Several limitations must be considered in the present study. First, we recorded $\mathrm{C}_{1} \mathrm{Pi}$ and VLa activity from only one monkey, and cortical data from a different monkey was used. The analysis described will have to be done with data from a second animal, combining $\mathrm{C}_{1} \mathrm{Pi}, \mathrm{VLa}$ and $\mathrm{M} 1$ recordings. In this second animal, it can also be investigated how single unit activity in M1 affects correlations between $\mathrm{C}_{1} \mathrm{Pi}$ and VLa. Furthermore, the number of single unit spike trains is limited for GPi and VLa, as we included only recordings in our analysis that contain single unit data in both $\mathrm{C}_{1} \mathrm{Pi}$ and VLa. Second, our analysis is based on very small time windows of $200 \mathrm{~ms}$ length. Data analysis methods, especially the frequency resolution, are restricted due to this small size, and hindered by firing rate modulations during movement, leading to varying control distributions. We cumulated correlations of all trials, but 
still, longer movement periods may be more appropriate to study movement-related correlation changes. A more complex and longer movement task could potentially also increase the role of the basal ganglia for such a movement and thus eventually information transmission from basal ganglia to thalamus. It is also possible that the basal ganglia play an evaluative role for movement, influencing subsequent movements [Feingold et al., 2015], which was not tested here.

Third, outward movements were cued by visual signals. These cues may have effects on basal ganglia activity themselves. A refined analysis is needed to decide whether the increases in discharge are time-locked to movement onset or to the visual stimulus. However, in $\mathrm{C}_{1} \mathrm{Pi}$, this has been done earlier [Turner and Anderson, 1997], where $70 \%$ of time-locking was to movement onset. In contrast, return movements were not cued. However, artifacts due to jaw movement could occur when reward was given, and return movements took place directly after reward. To estimate these effects, we calculated correlations also in the time period when reward was given. Fourth, we did not consider frequency specific effects yet. Our analysis is based on correlations in the time domain, whereas coherence is a common measure for neural communication [Fries, 2005]. Also more detailed analyses, for example involving Granger causality, may shed light into the interactions of basal ganglia and thalamus. Furthermore, we are particularly interested in the timing of bursts and the relation of burst occurrences in C.Pi and VLa. Last, we interpreted C.Pi activity as the main output of the basal ganglia. It is known that also the $\mathrm{SNr}$ can serve as an output nucleus, but mainly carries information of head movements rather than body movements [DeLong, 1983]. Also the interpretation of subcortical LFPs in general is not clear. While cortical LFPs seem to be mainly influenced by synaptic currents, evoking fields that sum up due to a cortical structure, this assumption might not hold for the basal ganglia [Buzsáki et al., 2012]. Here, we did not find stronger correlations between C.Pi spikes with VLa LFPs than between VLa spikes with GPi LFPs. Thus, it is not clear if this is due to missing input from GPi to VLa, or due to a misleading interpretation of the LFP in VLa.

We are not the first to describe qualitative differences between basal ganglia and cortical activity. It is a widely discussed phenomenon that the healthy globus pallidus, in particular its external part (CPe), does not show significant correlations in single unit activity [Nini et al., 1995, Bar-Gad et al., 2003]. Goldberg et al. [2004] already pointed out that spike-LFP coupling is higher in cortex than in G.P, and that this coupling increases in both brain areas in parkinsonism. However, Goldberg et al. [2004] did not investigate the influence of movement on spike-LFP coupling. Still, it has been mentioned that basal ganglia output stays uncorrelated even during pe- 
riods of movement [Bergman et al., 1998, Morris et al., 2003]. Since the movement periods in our experiments were very short, we cannot exclude that significant correlations within $\mathrm{C}_{\mathrm{P}} \mathrm{Pi}$ occur for longer periods of movement. In the time window "during movement", slight but not significant spike correlations occurred in C.Pi. C.Pi LFPs were highly enhanced in magnitude during movement, potentially indicating subtle synchrony of large numbers of neurons. Hence, it is possible that C.Pi activity is actually not qualitatively different to M1 activity, but characteristics like synchrony are just much weaker than in other structures.

Further, recent studies have reported that in healthy mammals, basal ganglia output does not directly entrain thalamic spiking. Anderson et al. [2015] described low spike-spike correlations between the rat $\mathrm{SNr}$ and the ventral anterior thalamus (VA) as well as low spike-LFP coupling. Also high-frequency stimulation of the $\mathrm{C}_{1} \mathrm{Pi}$ did not lead to entrainment of thalamic cells [Kammermeier et al., 2014], but only to subtle changes in oscillatory power. In contrast, entrainment of thalamic cells by basal ganglia input seems to be common in birds [Goldberg et al., 2012, Goldberg and Fee, 2012]. Computational models, however, have assumed that GiPi output has prominent cell-to-cell effects on thalamus, at least under parkinsonian conditions and in particular after the occurrence of bursts in G.Pi [Rubin and Terman, 2004, Guo et al., 2008, Guo and Rubin, 2011, Reitsma et al., 2011, Cagnan et al., 2009, Pirini et al., 2009, Meijer et al., 2011, Cleary et al., 2013]. Although we found high LFP correlations between $\mathrm{CPi}$ and VLa, it is not clear which physiological properties are actually reflected in these correlations, and whether they describe a transmission of information. M1 might also project directly to C.Pi [Naito and Kita, 1994, Milardi et al., 2014, Smith and Wichmann, 2014], and we found significant LFP correlations between $\mathrm{M} 1$ and $\mathrm{C}_{1} \mathrm{Pi}$, as we did between VLa and M1. However, timing of those correlations was different for GPi-M1 CCFs and VLa-M1 CCFs, and cortical input could only scarcely explain G.Pi-VLa correlations when using a partial spectra method. It thus seems unlikely that input from cortex is the main reason for high LFP correlations between CPi and VLa. Additionally, we reduced artifacts due to volume conduction by using reference electrodes within the same nuclei. Therefore, we assume that at least some parts of the LFP correlations between C.Pi and VLa are indicators of a functional connection between basal ganglia and thalamus.

Our knowledge on downstream effects of basal ganglia activity is patchy. Most evidence comes from birds [Goldberg et al., 2012, Goldberg and Fee, 2012], and recently rodents [Anderson et al., 2015]. However, few data on the primate basal gangliathalamic connection is available [Kammermeier et al., 2014, Zimnik and Turner, 2015]. This connection seems to differ highly between species: for example, interneurons 
are abundant in the primate thalamus [Ilinsky et al., 1997, Kultas-Ilinsky et al., 1997]. For the understanding of motor function of the basal ganglia, downstream effects on thalamus have a crucial role to show which part of basal ganglia activity is relevant for movement. To shed light into the importance of basal ganglia activity changes for PD, models of parkinsonism are needed to investigate how this information transmission changes after dopamine depletion. We expect that the communication between basal ganglia and thalamus differs in parkinsonism and potentially more information is transmitted to thalamus than in the healthy situation [Reitsma et al., 2011, Anderson et al., 2015]. In particular, we are curious how average noise correlations change and what happens to the coherence at high beta frequency (Fig. 5.5). It will furthermore be of interest if synchrony of basal ganglia output is increased during movement in parkinsonian animals, i.e. if the basal ganglia lose their ability to decorrelate movement-related inputs.

\begin{tabular}{|c|c|c|}
\hline Method & Result & Conclusion \\
\hline average spike properties & $\begin{array}{l}\text { different rate and burst proper- } \\
\text { ties in } M 1, C_{1} P i \text {, and VLa }\end{array}$ & $\begin{array}{l}\text { firing rates are in accordance } \\
\text { with literature; care is needed } \\
\text { when comparing nuclei }\end{array}$ \\
\hline average LFP spectra & $\begin{array}{l}\text { beta modulation with move- } \\
\text { ment in GPi, VLa, and M1 }\end{array}$ & $\begin{array}{l}\text { strong beta modulation in } \mathrm{GPi} \\
\text { has no dominant influence on } \\
\text { beta modulation in VLa }\end{array}$ \\
\hline spike-density averages & $\begin{array}{l}\text { movement-related discharge } \\
\text { significant in many units of } \\
\mathrm{GPi}, \mathrm{VLa} \text {, and M1 }\end{array}$ & $\begin{array}{l}\text { predominantly positive dis- } \\
\text { charge in both } \mathrm{GPi} \text { and VLa } \\
\text { argues against a gating of } \\
\text { thalamus by basal ganglia } \\
\text { output }\end{array}$ \\
\hline Spike CCFs & $\begin{array}{l}\text { very low spike coupling be- } \\
\text { tween } \mathrm{CPi} \text { and VLa }\end{array}$ & \multirow{4}{*}{$\begin{array}{l}\text { cell-to-cell interactions are } \\
\text { weak; information might be } \\
\text { transmitted via many neurons }\end{array}$} \\
\hline LFP CCFs \&r pCCFs & $\begin{array}{l}\text { high LFP coupling between } \\
\mathrm{GPi} \text { and VLa which is only } \\
\text { scarcely predicted by M1 }\end{array}$ & \\
\hline Spike-LFP coupling & low spike-LFP coupling & \\
\hline spike count correlations & $\begin{array}{l}\text { low noise correlations between } \\
\text { GPi and VLa, but in sum signif- } \\
\text { icant, especially in beta band }\end{array}$ & \\
\hline
\end{tabular}

Table 5.3: Overview of the most important findings. 


\section{Appendix}

\section{Motivation of Equation (5.5) for predicted cross-correlations}

Similar as Goldberg et al. [2004], we want to predict correlations from a third signal, namely an LFP signal. In contrast to Goldberg et al. [2004], we predict correlations between two LFP signals, and not between two single unit signals. In this appendix, we recapitulate the motivation of Equation (5.5) from Goldberg and Bergman [2005], based on Percival and Walden [1993], adapted to the prediction of LFP correlations.

We assume that each preprocessed LFP can be described by a real-valued zeromean second-order stationary process $X(t)$ that can be expressed as

$$
X(t)=\int_{-\infty}^{\infty} e^{i 2 \pi f t} d \tilde{X}(f)
$$

For details on this stochastic integral, see Priestley [1981]. The increment of the process in frequency domain, $\tilde{X}(f)$, and has the following properties:

(i) $E[d \tilde{X}(f)]=0$ for all $f$, where $E[\cdot]$ is the expectation operator;

(ii) $E\left[d \tilde{X}^{*}(f) d \tilde{X}\left(f^{\prime}\right)\right]=\delta_{f, f^{\prime}} \sigma_{X X}^{2}(f) d f$,

where $\delta_{f, f^{\prime}}$ is the Kronecker delta function, and $\sigma_{X X}^{2}$ the power spectrum of $X(t)$.

* indicates the complex conjugate.

Further, we assume that the LFP of neuron $i\left(L_{i}\right)$ has a time-invariant linear relationship to the source LFP $S$ of the form

$$
L_{i}(t)=\int_{-\infty}^{\infty} a_{i}(u) S(t-u) d u
$$

In the frequency domain, the increment of $\tilde{L}_{i}$ becomes

$$
\tilde{d} L_{i}(f)=\tilde{a}_{i}(f) d \tilde{S}(f)
$$

$a_{i}(u)$ are unknown functions that linearly relate the LFP to the source $S$. Civen that we use zero-mean LFPs, there is no constant term in Equation (5.10). We multiply Equation (5.11) with $d \tilde{S}^{*}(f)$, calculate the expectation value,

$$
E\left[d \tilde{L}_{i}(f) d \tilde{S}^{*}(f)\right]=\tilde{a}_{i}(f) E\left[d \tilde{S}(f) d \tilde{S}^{*}(f)\right]
$$


and use Property (ii) to find an expression for $\tilde{a}_{i}$ :

$$
\tilde{a}_{i} d f=\frac{E\left[d \tilde{L}_{i}(f) d \tilde{S}^{*}(f)\right]}{\sigma_{S S}^{2}(f)} .
$$

The CCF for continuous time is defined analogously to Equation (5.2):

$$
\operatorname{CCF}_{i, j}(\tau)=\frac{E\left[L_{i}(t) L_{j}(t+\tau)\right]}{R_{i j}^{2}},
$$

where the constant $R_{i j}^{2}=E\left[L_{i}(t) L_{j}(t)\right]$ normalizes the CCF. Since the LFPs are realvalued, we can replace $L_{i}(t)$ by $L_{i}^{*}(t)$. To find the pCCF, we estimate the CCF (Equation 5.14) by using LFPs linearly dependent on the source signal as described by Equation (5.10). Hence, we insert Equation (5.10) into Equation (5.14) and use Equations (5.9) and (5.11):

$$
p C C F_{i, j}(\tau)=\frac{1}{R_{i j}^{2}} \int_{-\infty}^{\infty} \int_{-\infty}^{\infty} e^{i 2 \pi\left[-f t+f^{\prime}(t+\tau)\right]} \tilde{a}_{i}(f)^{*} E\left[d \tilde{S}(f)^{*} d \tilde{S}\left(f^{\prime}\right)\right] \tilde{a}_{j}\left(f^{\prime}\right) .
$$

Using Property (ii) again, we can replace $E\left[d \tilde{S}(f)^{*} d \tilde{S}\left(f^{\prime}\right)\right]$ :

$$
p C C F_{i, j}(\tau)=\frac{1}{R_{i j}^{2}} \int_{-\infty}^{\infty} \tilde{a}_{i}^{*}(f) \sigma_{S S}^{2}(f) \tilde{a}_{j}(f) e^{i 2 \pi f \tau} d f .
$$

Finally, we insert Equation (5.13) and obtain

$$
p C C F_{i, j}(\tau)=\int_{-\infty}^{\infty} \frac{\chi_{i}(f) \chi_{j}^{*}(f)}{\sigma_{S S}^{2}(f)} \frac{R_{i S}^{2} R_{j S}^{2}}{R_{i j}^{2}} e^{i 2 \pi f \tau} d f
$$

with the cross-spectral densities

$$
\chi_{i}(f) d f=\frac{E\left[d \tilde{L}_{i}^{*}(f) d \tilde{S}(f)\right]}{R_{i S}^{2}} .
$$

Expressed in terms of Fourier transforms, this leads to Equation (5.5):

$$
p C C F_{L_{i}, L_{j}, S}=\mathcal{F}^{-1}\left(\frac{\mathcal{F}\left(C C F_{L_{i}, S}\right) \mathcal{F}\left(C C F_{S, L_{j}}\right)}{\left|\mathcal{F}\left(C C F_{S, S}\right)\right|}\right),
$$


ChAPTER 6

\section{Conclusion}

With this thesis, we want to provide insights into the role of gap junctions for the pathology of Parkinson's disease (PD). Our hypothesis on gap junction coupling in the external part of the globus pallidus ( $\mathrm{C}_{1} \mathrm{Pe}$ ) and its involvement in the pathophysiology of PD is novel. By using a combination of experiments, data analysis and computational modeling, we approached a deeper understanding of basal ganglia structure and dynamics, how the dynamics could be influenced by gap junction coupling and finally, on the impact of this basal ganglia activity. We had a specific hypothesis which suggested gap junction coupling in the C.Pe as a novel mechanism to disrupt desynchronization of this nucleus, and we provided first evidence for the occurrence of gap junctions in G.Pe. The GPe is ideally located to spread synchrony and oscillations to other parts of the basal ganglia. In our computational model, sparse pallidal gap junction coupling was able to synchronize basal ganglia activity. Lastly, we studied the relation between basal ganglia output and thalamic input activity, showing only weak cell-to-cell influences of the basal ganglia on downstream structures during a learned task. After all, a lot of questions remain open.

\section{$P D$ is a dynamic disease}

The pathology of PD has various sides. One of these sides are the motor symptoms, varying with each patient and his current condition. Motor symptoms of PD can be highly variable, and the same is true for neural activity in the basal ganglia. We call PD a "dynamic disease": neural dynamics of the basal ganglia might be highly important to describe the present condition of the patient. Various studies have shown how dynamic and state-dependent basal ganglia activity is [Magill et al., 2006, Cagnan et al., 2015, Feingold et al., 2015]. Although average properties of neural activity, for example beta oscillations, have shown great potential as biomarkers, it is not clear how short-term temporal variations of such biomarkers correlate or even causally 
relate to motor performance. For example, short episodes of beta oscillations are also common in the healthy basal ganglia (Chapter 5).

Providing a link between the current state of basal ganglia activity and motor control would be a major step towards a better understanding of PD motor symptoms. In particular, it would strengthen the role of computational studies that model the dynamic modulation of biomarkers. On the other hand, computational studies are only beginning to address the question how beta episodes in the heathy brain are decorrelated after some milliseconds, why this decorrelation fails and long beta periods emerge (Chapter 4), and in which way such long beta episodes influence motor control. Many different mechanisms might be able to explain such waxing and waning of correlations as well as their temporal fixation in the parkinsonian basal ganglia, and it seems to be a crucial question which of those possible mechanisms are of pathophysiological importance.

\section{Gap junction coupling might be increased in PD}

In Chapter 3, we described the finding of the gap junction protein connexin-36 (Cx36) in the human pallidum. It is not completely clear how the occurrence of Cx36 relates to the existence of functional gap junctions, but typically Cx36 is seen as a first indication that gap junctions can exist [Belluardo et al., 1999]. Furthermore, we saw a remodeling of Cx36 in PD patients, showing higher levels of this protein than control subjects. Since dopamine has been described to modulate gap junction conductance in the retina [Hampson et al., 1992, Li et al., 2013], it seems likely that gap junctions in the basal ganglia are sensitive to dopamine depletion in PD. Phookan et al. [2015] described a depression of beta oscillations after blocking gap junctions in C.Pe. As broad band gap junction blockers also affect gap junction coupling between glia cells and come along with non-specific side effects, it is not clear how this relates to our results. Still, the results of Phookan et al. [2015] show how high the impact of gap junctions on basal ganglia activity might be. Notably, systemic application of gap junction blockers reduced beta oscillations similarly as their local application to the C.Pe [Phookan et al., 2015], suggesting that also gap junctions in other areas of the brain might be affected.

Gap junctions exist between fast-spiking interneurons (FSIs) of striatum [Kita et al., 1990], and dopamine depletion in PD patients is most prominent in striatum [Rajput et al., 2008]. We also saw an increase in Cx36 puncta in the striatum of PD patients compared to control subjects. Dye-coupling, an indicator of gap junc- 
tion coupling, was increased between striatal medium-spiny neurons (MSNs) of 6hydroxydopamine (6-OHDA) treated rats [Onn and Grace, 1999], although gap junction coupling between MSNs is not proven yet. Onn and Grace [1999] used Lucifer Yellow as a dye, which, due to its size, is normally not considered to pass through Cx36 gap junctions, as present between FSIs. Hence, Onn and Cirace [1999] were not able to describe dye coupling between FSIs. The nature of gap junction coupling thus remains unclear even in striatum, where a modulation of FSI gap junction coupling after dopamine depletion still remains to be shown. Consequently, pallidal gap junctions are even harder to study. While it seemed difficult to obtain paired recordings for inhibitory connections within GPe [Bugaysen et al., 2013], the situation will become even more problematic for gap junctions since their occurrence is expected to be very sparse. Dye-coupling in combination with patch-clamp experiments might be a solution to find pairs of neurons coupled via gap junctions. However, such experiments would still be complex. It thus remains a problem to estimate the actual level of gap junction coupling in-vivo. This is, however, also the case for other connectivities like inhibition. As, in general, little is known on basal ganglia micro-circuitries and how those circuitries shape activity, novel techniques like optogenetics might shed light into the roles of certain types of basal ganglia subnetworks, e. g., within G.Pe [Mastro et al., 2014].

\section{Synchrony in the basal ganglia can be orchestrated by pallidal connectivity}

Mechanistic computational modeling can be used to relate structure to function. We chose a neural network model of the basal ganglia to study the effects of pallidal gap junctions (Chapter 4). While sparse inhibition between C.Pe neurons desynchronized activity, sparse gap junction coupling had a desynchronizing effect only at very low strengths, but synchronized when they became stronger. This occurred in both small and larger network models and was independent of the specific synaptic architecture. We described in Chapter 2 that the CPe might be an important hub within the basal ganglia, controlling the levels of synchrony in many nuclei. Also in our model, the effects of pallidal connectivity spread to the neighboring nuclei STN and $\mathrm{C}_{1} \mathrm{Pi}$, and it is possible that this is also the case for nuclei that were not present in our model. For example, striatum is densely innervated by C.Pe axons [Mallet et al., 2012, Fujiyama et al., 2015], building the basis for a feedback loop between G.Pe and striatum. The network with low gap junction coupling was able to decorrelate basal ganglia activity, while the network with high gap junction coupling showed sustained epochs of coherent beta activity. Thus, increased gap junction coupling in 
the parkinsonian basal ganglia might also make them prone to oscillate with beta oscillations from higher structures.

Still, a lot of microscopic detail is lacking in this model. For example, neuron models were highly simplified, consisting of only one compartment without axons or dendrites. Also different neuron types within G.Pe have been described [Mallet et al., 2012, Mastro et al., 2014, Dodson et al., 2015, Abdi et al., 2015], but we used only one neural population within G.Pe. Notably, the distinct neural populations in $C_{1}$ Pe have different projection sites [Mallet et al., 2012]. First, it was assumed that one GPe population, termed "arkypallidal" neurons, projects to striatum, and the other population, termed "prototypic" neurons, projects to downstream nuclei including STN [Mallet et al., 2012]. However, a recent study demonstrated that also connections from prototypic neurons to striatum exist [Fujiyama et al., 2015]. Since arkypallidal neurons are nevertheless assumed to project only to striatum, these neurons might have a special role in a feedback loop between striatum and C.Pe. An early study using electron microscopy [Kita, 1994] found gap junctions on parvalbumin-negative (arkypallidal) neurons, suggesting that the effects of gap junction coupling between such neurons might be higher in striatum than in other neighboring nuclei. A lot of microscopic detail is known for striatum, and has also been incorporated into computational models. For example, Damodaran et al. [2015] described the effects of gap junctions between FSIs on striatal dynamics, showing that synchronization of FSIs by gap junctions is crucial for the maintenance of beta oscillations. Still, many more factors, like inhibition between FSIs and from FSIs to MSNs, determined the state of the system, and it is not clear what effects the cell models had. This examplifies a typical difficulty when working with network models - it is hard to find out which details are crucial for network dynamics and which details can be neglected. Various different settings have to be tested to get a feeling how sensitive the model is. In the case of the basal ganglia, another problem is that much microscopic detail is not even known.

\section{Effects of physiological basal ganglia activity on downstream structures are weak on a cell-to-cell basis}

Lastly, in Chapter 5, we investigated the physiological impact of basal ganglia activity in a monkey, by looking at its downstream effects during a simple, learned reaching task. Such recordings, in particular including both single unit and local field potential (LFP) data, have not been available until now. Contrary to earlier experiments in songbirds, we did not find evidence for a direct entrainment of thalamic 
activity by basal ganglia inputs, but thalamic unit activity showed only very subtle modification after spiking in the internal part of the globus pallidus (C.Pi). Still, strong LFP correlations between basal ganglia and thalamus suggested that many subtle correlations on the cell level could sum up together to a cumulative influence. We also did not find synchrony and spike-field coupling in basal ganglia output activity. However, if those characteristics are just even lower in the basal ganglia than in cortex, they might not be detectable in a restricted amount of recordings, but only show up for very large numbers of neurons. Due to technical limitations, so many recordings are not available for the basal ganglia-thalamic connection. Again, we can interpret the LFP as the superposition of activity from many neurons, and LFP power is highly increased in $\mathrm{C}_{1} \mathrm{Pi}$ during movement. This huge increase in LFP power could be an indication of slight correlations between many neurons. Unfortunately, the interpretation of LFPs in the basal ganglia is uncertain. While cortical LFPs have been studied extensively, and might primarily be influenced by synaptic currents, it remains speculative if this also holds true for $\mathrm{C}_{\mathrm{Pi}}$, where a structured organization of dendritic connections is missing [Buzsáki et al., 2012]. Furthermore, it is non-trivial to separate movement-related changes in activity from other effects, e. g., due to reward or cueing. We did not do a refined analysis yet relating movement parameters or electromyographic (EMG) data to neural activity.

\section{The basal ganglia are an enigma}

Both basal ganglia structure and function remain nebulous. While much is known on cortical connectivity, function and relation to behavior, such details are widely missing for the basal ganglia. For example, coding of movements in motor cortex is quite well understood. Based on the information of motor cortex discharge, we can already make predictions on the movement itself [Georgopoulos et al., 1986]. We are far from having such a relation for the basal ganglia, and we do not even know when precisely movement-related inputs from the basal ganglia enter the motor cortex. Also the microscopic structure of basal ganglia nuclei such as pallidum or subthalamic nucleus is barely known, as discussed above. Even connections between nuclei are still being discovered, like a potential connection from motor cortex to pallidum [Milardi et al., 2014], or a dense innervation of pallidal projections to striatum [Fujiyama et al., 2015]. Getting a deeper understanding of both basal ganglia structure and function can teach us what the basal ganglia really do, and what might go wrong in PD. This kind of knowledge forms the basis for the development of novel treatments for PD, be it medication or surgery. With regard to our results, a possible treatment complementing L-Dopa could be gap junction blockers. However, we are still in the 
beginning of understanding the roles of gap junctions in the basal ganglia, and it is far to early to investigate the effects of such a medication.

"[...] You think you can hear it rumbling, but you have a lot of time to think. And so you just don't live that moment of the bus hitting you until it happens. There's all kinds of room in that space." 1

\footnotetext{
1 Quote, Interview with Michael J. Fox in "Good Houskeeping", May 2011, on dealing with the situation when you're stuck in the middle of the street in cement shoes, and the bus is coming at you.
} 


\section{References}

Abdi, A., Mallet, N., Mohamed, F. Y., Sharott, A., Dodson, P. D., Nakamura, K. C., Suri, S., Avery, S. V., Larvin, J. T., Garas, F. N., Garas, S. N., Vinciati, F., Morin, S., Bezard, E., Baufreton, J. and Magill, P. J. (2015) Prototypic and Arkypallidal Neurons in the Dopamine-Intact External Globus Pallidus, The Journal of Neuroscience, 35(17), 6667-6688.

Abedi, P. M., Delaville, C., De Deurwaerdere, P., Benjelloun, W. and Benazzouz, A. (2013) Intrapallidal administration of 6-hydroxydopamine mimics in large part the electrophysiological and behavioral consequences of major dopamine depletion in the rat, Neuroscience, 236, 289-297.

Adelman, J. P., Maylie, J. and Sah, P. (2012) Small-conductance Ca2+-activated K+ channels: form and function, Annual Review of Physiology, 74, 245-269.

Alexander, G. E., DeLong, M. R. and Strick, P. L. (1986) Parallel organization of functionally segregated circuits linking basal ganglia and cortex, Annual Review of Neuroscience, 9(1), 357-381.

Alexander, G. E. and Crutcher, M. D. (1990) Functional architecture of basal ganglia circuits: Neural substrates of parallel processing, Trends in Neurosciences, 13(7), 266-271.

Anderson, C. J., Sheppard, D. T., Huynh, R., Anderson, D. N., Polar, C. A. and Dorval, A. D. (2015) Subthalamic deep brain stimulation reduces pathological information transmission to the thalamus in a rat model of parkinsonism, Frontiers in Neural Circuits, 9 (31).

Angeli, A., Akram, H., Zacharia, A., Limousin, P., Hariz, M., Zrinzo, L. and Foltynie, T. (2015) Varying time-course of effects of high frequency stimulation of sub-regions of the globus pallidus in patients with parkinson's disease, Parkinsonism \& related disorders, 21(6), 597-602.

Bar-Gad, I., Heimer, G., Ritov, Y. and Bergman, H. (2003) Functional correlations between neighboring neurons in the primate globus pallidus are weak or nonexistent, Journal of Neuroscience, 23(10), 4012-4016.

Barbiroli, B., lotti, S., Cortelli, P., Martinelli, P., Lodi, R., Carelli, V. and Montagna, P. (1999) Low brain intracellular free magnesium in mitochondrial cytopathies, Journal of Cerebral Blood Flow E Metabolism, 19(5), 528-532.

Bargiotas, P., Muhammad, S., Rahman, M. et al. (2012) Connexin 36 promotes cortical spreading depolarization and ischemic brain damage, Brain research, 1479, 80-85.

Baufreton, J., Kirkham, E., Atherton, J. F., Menard, A., Magill, P. J., Bolam, J. P. and Bevan, M. D. (2009) Sparse but selective and potent synaptic transmission from the globus pallidus to the subthalamic nucleus, Journal of Neurophysiology, 102(1), 532-545.

Bautista, W., Rash, J. E., Vanderpool, K. G., Yasumura, T. and Nagy, J. I. (2014). Re-evaluation of connexins associated with motoneurons in rodent spinal cord, sexually dimorphic motor nuclei and trigeminal motor nucleus, European Journal of Neuroscience, 39(5), 757-770. 
Belluardo, N., Trovato-Salinaro, A., Mudo, G., Hurd, Y. L. and Condorelli, D. F. (1999) Structure, chromosomal localization, and brain expression of human Cx36 gene, Journal of Neuroscience Research, 57(5), 740-752.

Belousov, A. B. (2012) Novel model for the mechanisms of glutamate-dependent excitotoxicity: Role of neuronal gap junctions, Brain research, 1487, 123-130.

Benarroch, E. E. (2013) HCN channels Function and clinical implications, Neurology, 80(3), 304-310.

Benhamou, L., Bronfeld, M., Bar-Gad, I. and Cohen, D. (2012) Gilobus Pallidus External Segment Neuron Classification in Freely Moving Rats: A Comparison to Primates, PloS one, 7(9), e45421.

Bennett, M. V. and Zukin, R. S. (2004) Electrical coupling and neuronal synchronization in the mammalian brain, Neuron, 41(4), 495-511.

Bergman, H., Wichmann, T., Karmon, B. and DeLong, M. R. (1994) Parkinsonian tremor is associated with low frequency neuronal oscillations in selective loops of the basal ganglia, The Basal Cranglia IV, 41, 317-325.

Bergman, H., Feingold, A., Nini, A., Raz, A., Slovin, H., Abeles, M. and Vaadia, E. (1998) Physiological aspects of information processing in the basal ganglia of normal and parkinsonian primates, Trends in Neurosciences, 21(1), 32-38.

Bevan, M. D., Magill, P. J., Terman, D., Bolam, J. P. and Wilson, C. J. (2002) Move to the rhythm: Oscillations in the subthalamic nucleus-external globus pallidus network, Trends in Neurosciences, 25(10), 525-531.

Bond, C. T., Maylie, J. and Adelman, J. P. (1999) Small-Conductance Calcium-Activated Potassium Channels, Annals of the New York Academy of Sciences, 868(1), 370-378.

Boraud, T., Bezard, E., Bioulac, B. and Gross, C. E. (2000) Ratio of inhibited-to-activated pallidal neurons decreases dramatically during passive limb movement in the MPTP-treated monkey, Journal of Neurophysiology, 83(3), 1760-1763.

Bosch-Bouju, C., Hyland, B. I. and Parr-Brownlie, L. C. (2013) Motor thalamus integration of cortical, cerebellar and basal ganglia information: implications for normal and parkinsonian conditions, Frontiers in Computational Neuroscience, 7 (163).

Bosch-Bouju, C., Smither, R. A., Hyland, B. I. and Parr-Brownlie, L. C. (2014) Reduced Reach-Related Modulation of Motor Thalamus Neural Activity in a Rat Model of Parkinson's Disease, The Journal of Neuroscience, 34(48), 15836-15850.

Boyes, J. and Bolam, J. P. (2007). Localization of GABA receptors in the basal ganglia, Progress in Brain Research, 160, 229-243.

Brittain, J. S., Probert-Smith, P., Aziz, T. Z. and Brown, P. (2013) Tremor suppression by rhythmic transcranial current stimulation, Current Biology, 23(5), 436-440.

Brittain, J. S. and Brown, P. (2013) Oscillations and the basal ganglia: Motor control and beyond, Neurolmage, 85, 637-647.

Brotchie, P., lansek, R. and Horne, M. K. (1991) Motor function of the monkey globus pallidus, Brain, 114(4), 1685-1702.

Brown, P., Oliviero, A., Mazzone, P. Insola, A. Tonali, P. and Di Lazzaro, V. (2001) Dopamine dependency of oscillations between subthalamic nucleus and pallidum in Parkinson's disease, Journal of Neuroscience, 21(3), 1033-1038.

Brown, P. (2003) Oscillatory nature of human basal ganglia activity: Relationship to the pathophysiology of Parkinson's disease, Movement Disorders, 18(4), 357-363.

Bugaysen, J., Bronfeld, M., Tischler, H., Bar-Gad, I. and Korngreen, A. (2010) Electrophysiological characteristics of globus pallidus neurons, PloS one, 5(8), e12001. 
Bugaysen, J., Bar-Gad, I. and Korngreen, A. (2013) Continuous Modulation of Action Potential Firing by a Unitary CABAergic Connection in the Globus Pallidus In Vitro, The Journal of Neuroscience, 33(31), 12805-12809.

Bukauskas, F. F., Jordan, K., Bukauskiene, A., Bennett, M. V., Lampe, P. D., Laird, D. W. and Verselis, V. K. (2000) Clustering of connexin 43-enhanced green fluorescent protein gap junction channels and functional coupling in living cells, Proceedings of the National Academy of Sciences, 97(6), 2556-2561.

Buzsáki, G., Anastassiou, C. A. and Koch, C. (2012) The origin of extracellular fields and currents EEG, ECoG, LFP and spikes, Nature Reviews Neuroscience, 13(6), 407-420.

Cagnan, H., Meijer, H. G., Van Gils, S. A., Krupa, M., Heida, T., Rudolph, M., Wadman, W. J. and Martens, H. C. (2009) Frequency-selectivity of a thalamocortical relay neuron during Parkinson's disease and deep brain stimulation: a computational study, European Journal of Neuroscience, 30(7), 1306-1317.

Cagnan, H., Duff, E. P. and Brown, P. (2015) The relative phases of basal ganglia activities dynamically shape effective connectivity in Parkinson's disease, Brain, 138(6), 1667-1678.

Catalan, M. J., Ishii, K., Honda, M., Samii, A. and Hallett, M. (1999) A PET study of sequential finger movements of varying length in patients with Parkinson's disease. Brain, 122(3), 483-495.

Cepeda, C., Walsh, J. P., Hull, C. D., Howard, S. G., Buchwald, N. A. and Levine, M. S. (1989) Dyecoupling in the neostriatum of the rat: I. Modulation by dopamine-depleting lesions, Synapse, 4(3), 229-237.

Chan, C. S., Shigemoto, R., Mercer, J. N. and Surmeier, D. J. (2004) HCN2 and HCN1 channels govern the regularity of autonomous pacemaking and synaptic resetting in globus pallidus neurons, The Journal of Neuroscience, 24(44), 9921-9932.

Chan, C. S., G.lajch, K. E., Gertler, T. S., Guzman, J. N., Mercer, J. N., Lewis, A. S., Goldberg, A. B., Tkatch, T., Shigemoto, R., Fleming, S. M., Chetkovich, D. M., Osten, P., Kita, H. and Surmeier, D. J. (2011). HCN channelopathy in external globus pallidus neurons in models of Parkinson's disease, Nature Neuroscience, 14(1), 85-92.

Chang, H. T., Kita, H. and Kitai, S. T. (1983) The fine structure of the rat subthalamic nucleus: an electron microscopic study, Journal of Comparative Neurology, 221(1), 113-123.

Chen, C. C., Litvak, V., Gilbertson, T., Kühn, A., Lu, C. S., Lee, S. T., Tsaie, C. H., Tischa, S., Limousina, P., Hariz, M. and Brown, P. (2007) Excessive synchronization of basal ganglia neurons at $20 \mathrm{~Hz}$ slows movement in Parkinson's disease, Experimental Neurology, 205(1), 214-221.

Chevalier, G. and Deniau, J. M. (1990) Disinhibition as a basic process in the expression of striatal functions, Trends in Neurosciences, 13(7), 277-280.

Chow, C. C. and Kopell, N. (2000) Dynamics of spiking neurons with electrical coupling, Neural Computation, 12(7), 1643-1678.

Chu, H. Y., Atherton, J. F., Wokosin, D., Surmeier, D. J. and Bevan, M. D. (2015) Heterosynaptic Regulation of External Gilobus Pallidus Inputs to the Subthalamic Nucleus by the Motor Cortex, Neuron, 85(2), 364-376.

Cleary, D. R., Raslan, A. M., Rubin, J. E., Bahgat, D., Viswanathan, A., Heinricher, M. M. and Burchiel, K. J. (2013) Deep brain stimulation entrains local neuronal firing in human globus pallidus internus, Journal of Neurophysiology, 109(4), 978-987.

Connelly, W. M., Schulz, J. M., Lees, G. and Reynolds, J. N. (2010) Differential short-term plasticity at convergent inhibitory synapses to the substantia nigra pars reticulata, The Journal of Neuroscience, 30(44), 14854-14861.

Connors, B. W. and Long, M. A. (2004) Electrical synapses in the mammalian brain, Annual Review of Neuroscience, 27, 393-418. 
Cooper, A. J. and Stanford, I. M. (2000) Electrophysiological and morphological characteristics of three subtypes of rat globus pallidus neurone in vitro, The Journal of Physiology, 527(2), 291-304.

Cooper, A. J. and Stanford, I. M. (2002) Calbindin D-28k positive projection neurones and calretinin positive interneurones of the rat globus pallidus, Brain Research, 929(2), 243-251.

Curti, S., Hoge, G., Nagy, J. I. and Pereda, A. E. (2012) Synergy between electrical coupling and membrane properties promotes strong synchronization of neurons of the mesencephalic trigeminal nucleus, The Journal of Neuroscience, 32(13), 4341-4359.

Damodaran, S., Evans, R. C. and Blackwell, K. T. (2014) Synchronized firing of fast-spiking interneurons is critical to maintain balanced firing between direct and indirect pathway neurons of the striatum, Journal of Neurophysiology, 111, 836-848.

Damodaran, S., Cressman, J. R., Jedrzejewski-Szmek, Z. and Blackwell, K. T. (2015) Desynchronization of Fast-Spiking Interneurons Reduces Beta Band Oscillations and Imbalance in Firing in the Dopamine-Depleted Striatum, The Journal of Neuroscience, 35(3), 1149-1159.

Deffains, M. and Bergman, H. (2015). Striatal cholinergic interneurons and cortico-striatal synaptic plasticity in health and disease, Movement Disorders, 30(8), 1014-1025.

Deister, C. A., Chan, C. S., Surmeier, D. J. and Wilson, C. J. (2009) Calcium-activated SK channels influence voltage-gated ion channels to determine the precision of firing in globus pallidus neurons, The Journal of Neuroscience, 29(26), 8452-8461.

Deister, C. A., Dodla, R., Barraza, D., Kita, H. and Wilson, C. J. (2013) Firing rate and pattern heterogeneity in the globus pallidus arise from a single neuronal population, Journal of Neurophysiology, 109(2), 497-506.

Dejean, C., Nadjar, A., Le Moine, C., Bioulac, B., Crross, C. E. and Boraud, T. (2012) Evolution of the dynamic properties of the cortex-basal ganglia network after dopaminergic depletion in rats, Neurobiology of disease, 46(2), 402-413.

Delong, M. R., Crutcher, M. D. and Georgopoulos, A. P. (1983) Relations between movement and single cell discharge in the substantia nigra of the behaving monkey, Journal of Neuroscience, 3(8), 1599-606.

DeLong, M. R. and Wichmann, T. (2007) Circuits and circuit disorders of the basal ganglia, Archives of Neurology, 64(1), 20-24.

DeLong, M. R. and Wichmann, T. (2015) Basal Ganglia Circuits as Targets for Neuromodulation in Parkinson's Disease, JAMA Neurology, 1-7.

Deniau, J. M. and Chevalier, G. (1985) Disinhibition as a basic process in the expression of striatal functions. II. The striato-nigral influence on thalamocortical cells of the ventromedial thalamic nucleus, Brain Research, 334(2), 227-233.

Dere, E. (2012) Gap junctions in the brain, Elsevier.

DeLong, M. R. (1971) Activity of pallidal neurons during movement, Journal of neurophysiology, 34(3), 414-427.

Dhooge, A., Govaerts, W. and Kuznetsov, Y. A. (2003) MATCONT: a MATLAB package for numerical bifurcation analysis of ODEs, ACM Transactions on Mathematical Software (TOMS), 29(2), 141 164.

Dodson, P. D., Larvin, J. T., Duffell, J. M., Garas, F. N., Doig, N. M., Kessaris, N., Duguid, I. C., Bogacz, R.Journal of Neuroscience, Butt, S. J. B., and Magill, P. J. (2015) Distinct Developmental Origins Manifest in the Specialized Encoding of Movement by Adult Neurons of the External Cilobus Pallidus, Neuron, 86(2), 501-513.

Draguhn, A., Traub, R. D., Schmitz, D. and Jefferys, J. G. R. (1998) Electrical coupling underlies high-frequency oscillations in the hippocampus in vitro, Nature, 394(6689), 189-192. 
Edgerton, J. R. and Jaeger, D. (2011) Dendritic sodium channels promote active decorrelation and reduce phase locking to parkinsonian input oscillations in model globus pallidus neurons, The Journal of Neuroscience, 31(30), 10919-10936.

Engel, A. K. and Singer, W. (2001) Temporal binding and the neural correlates of sensory awareness, Trends in Cognitive Sciences, 5(1), 16-25.

Engel, A. K. and Fries, P. (2010) Beta-band oscillations - signalling the status quo?, Current Opinion in Neurobiology, 20(2), 156-165.

Eusebio, A., Cagnan, H. and Brown, P. (2007) Does suppression of oscillatory synchronisation mediate some of the therapeutic effects of DBS in patients with Parkinson's disease?, Frontiers in Integrative Neuroscience, 6(47).

Fan, K. Y., Baufreton, J., Surmeier, J. D., Chan, C. S. and Bevan, M. D. (2013) Proliferation of external globus pallidus-subthalamic nucleus synapses following degeneration of midbrain dopamine neurons, Journal of Neuroscience, 32(40), 13718-13728.

Feingold, J., Cibson, D. J., DePasquale, B. and Ciraybiel, A. M. (2015) Bursts of beta oscillation differentiate postperformance activity in the striatum and motor cortex of monkeys performing movement tasks, Proceedings of the National Academy of Sciences, 112(44), 13687-13692.

Fernandez-Suarez, D., Celorrio, M., Lanciego, J. L., Franco, R. and Aymerich, M. S. (2012) Loss of parvalbumin-positive neurons from the globus pallidus in animal models of Parkinson disease, Journal of Neuropathology and Experimental Neurology, 71(11), 973-982.

Filion, M., Tremblay, L. and Bédard, P. J. (1988) Abnormal influences of passive limb movement on the activity of globus pallidus neurons in parkinsonian monkeys, Brain Research, 444(1), 165-176.

Filion, M., and Paul, J. B. (1989) Responses of pallidal neurons to striatal stimulation in monkeys with MPTP-induced parkinsonism, Brain Research, 498(1), 17-33.

Fortier, P. A. (2010) Detecting and estimating rectification of gap junction conductance based on simulations of dual-cell recordings from a pair and a network of coupled cells, Journal of Theoretical Biology, 265(2), 104-114.

Franco, V. and Turner, R. S. (2012) Testing the contributions of striatal dopamine loss to the genesis of parkinsonian signs, Neurobiology of Disease, 47(1), 114-125.

François, C., Percheron, G., Yelnik, J. and Heyner, S. (1984) A Golgi analysis of the primate globus pallidus. I. Inconstant processes of large neurons, other neuronal types, and afferent axons, Journal of Comparative Neurology, 227(2), 182-199.

Fries, P. (2005) A mechanism for cognitive dynamics: neuronal communication through neuronal coherence, Trends in Cognitive Sciences, 9(10), 474-480.

Fujita, T., Fukai, T. and Kitano, K. (2012) Influences of membrane properties on phase response curve and synchronization stability in a model globus pallidus neuron, Journal of Computational Neuroscience, 32(3), 539-553.

Fujiyama, F., Nakano, T., Matsuda, W., Furuta, T., Udagawa, J. and Kaneko, T. (2015) A singleneuron tracing study of arkypallidal and prototypic neurons in healthy rats, Brain Structure and Function, 1-8.

Fukuda, T., Kosaka, T., Singer, W. and Galuske, R. A. (2006) Gap junctions among dendrites of cortical GABAergic neurons establish a dense and widespread intercolumnar network. The Journal of Neuroscience, 26(13), 3434-3443.

Fukuda, T. (2009) Network architecture of gap junction-coupled neuronal linkage in the striatum, The Journal of Neuroscience, 29(4), 1235-1243.

Galarreta, M. and Hestrin, S. (1999) A network of fast-spiking cells in the neocortex connected by electrical synapses, Nature, 402(6757), 72-75. 
Galarreta, M. and Hestrin, S. (2001) Electrical synapses between GABA-releasing interneurons, Nature Reviews Neuroscience, 2(6), 425-433

Cittis, A. H., Berke, J. D., Bevan, M. D., Chan, C. S., Mallet, N., Morrow, M. M., and Schmidt, R. (2014) New roles for the external globus pallidus in basal ganglia circuits and behavior, The Journal of Neuroscience, 34(46), 15178-15183.

Govaerts, W. and Sautois, B. (2006). Computation of the phase response curve: a direct numerical approach, Neural Computation, 18(4), 817-847.

Hestrin, S. and Galarreta, M. (2005) Electrical synapses define networks of neocortical GABAergic neurons, Trends in Neurosciences, 28(6), 304-309.

Galvan, A., Floran, B., Erlij, D. and Aceves, J. (2001) Intrapallidal dopamine restores motor deficits induced by 6-hydroxydopamine in the rat, Journal of Neural Transmission, 108, 153-166.

Galvan, A., Villalba, R. M., West, S. M., Maidment, N. T., Ackerson, L. C., Smith, Y. and Wichmann, T. (2005) GABAergic modulation of the activity of globus pallidus neurons in primates: in vivo analysis of the functions of $G_{1} A B A$ receptors and $C_{1} A B A$ transporters, Journal of Neurophysiology, 94(2), 990-1000.

Galvan, A., Hu, X., Smith, Y. and Wichmann, T. (2010) Localization and function of GABA transporters in the globus pallidus of parkinsonian monkeys, Experimental Neurology, 223(2), 505-515.

Galvan, A., Hu, X., Smith, Y. and Wichmann, T. (2011) Localization and pharmacological modulation of $\mathrm{C}_{\mathrm{A}} \mathrm{ABA}-\mathrm{B}$ receptors in the globus pallidus of parkinsonian monkeys, Experimental Neurology, 229, 429-439.

Galvan, A., Devergnas, A. and Wichmann, T. (2015) Alterations in neuronal activity in basal gangliathalamocortical circuits in the parkinsonian state, Frontiers in Neuroanatomy, 9.

Gao, M., Wang, H. L., Wang, L. C. and Chen, X. W. (2013) Modulation of connexin 36 expression in basal ganglia and motor cortex in rat model of Parkinson's disease, Chinese Journal of Contemporary Neurology and Neurosurgery, 13(8), 697-703.

Georgopoulos, A. P., Schwartz, A. B. and Kettner, R. E. (1986) Neuronal population coding of movement direction, Science, 233(4771), 1416-1419.

Gibson, J. R., Beierlein, M. and Connors, B. W. (1999) Two networks of electrically coupled inhibitory neurons in neocortex, Nature, 402(6757), 75-79.

Gittis, A. H., Hang, G. B., LaDow, E. S., Shoenfeld, L. R., Atallah, B. V., Finkbeiner, S. and Kreitzer, A. C. (2011) Rapid target-specific remodeling of fast-spiking inhibitory circuits after loss of dopamine, Neuron, 71(5), 858-868.

Goldberg, J. A., Kats, S. S. and Jaeger, D. (2003) Globus pallidus discharge is coincident with striatal activity during global slow wave activity in the rat, Journal of Neuroscience, 23(31), 10058-10063.

Goldberg, J. A., Rokni, U., Boraud, T., Vaadia, E. and Bergman, H. (2004) Spike synchronization in the cortex-basal ganglia networks of parkinsonian primates reflects global dynamics of the local field potentials, The Journal of Neuroscience, 24(26), 6003-6010.

Goldberg, J. A. and Bergman, H. (2005) A Partial Spectra Method for Predicting Spike Correlations from Local Field Potentials, The Basal Canglia VIII, Springer US, 47-53.

Coldberg, J. A. and Bergman, H. (2011) Computational physiology of the neural networks of the primate globus pallidus: function and dysfunction, Neuroscience, 198, 171-192.

Goldberg, J. H., Farries, M. A. and Fee, M. S. (2012) Integration of cortical and pallidal inputs in the basal ganglia-recipient thalamus of singing birds, Journal of Neurophysiology, 108(5), 1403-1429.

Cooldberg, J. H. and Fee, M. S. (2012) A cortical motor nucleus drives the basal ganglia-recipient thalamus in singing birds, Nature Neuroscience, 15(4), 620-627. 
Goldberg, J. A., Atherton, J. F. and Surmeier, D. J. (2013) Spectral reconstruction of phase response curves reveals the synchronization properties of mouse globus pallidus neurons, Journal of Neurophysiology, 110(10), 2497-2506.

Gradinaru, V., Mogri, M., Thompson, K. R., Henderson, J. M. and Deisseroth, K. (2009) Optical deconstruction of parkinsonian neural circuitry, Science, 324(5925), 354-359.

Ciraybiel, A. M., Aosaki, T., Flaherty, A. W. and Kimura, M. (1994) The basal ganglia and adaptive motor control, Science, 265(5180), 1826-1831.

Cünay, C., Edgerton, J. R. and Jaeger, D. (2008) Channel density distributions explain spiking variability in the globus pallidus: A combined physiology and computer simulation database approach, The Journal of Neuroscience, 28(30), 7476-7491.

Guo, Y., Rubin, J. E., Mclntyre, C. C., Vitek, J. L. und Terman, D. (2008) Thalamocortical relay fidelity varies across subthalamic nucleus deep brain stimulation protocols in a data-driven computational model, Journal of Neurophysiology, 99(3), 1477-1492.

Guo, Y. and Rubin, J. E. (2011) Multi-site stimulation of subthalamic nucleus diminishes thalamocortical relay errors in a biophysical network model, Neural Networks, 24(6), 602-616.

Haas, J. S., Zavala, B. and Landisman, C. E. (2011) Activity-dependent long-term depression of electrical synapses, Science, 334(6054), 389-393.

Hahn, P. J. and Mclntyre, C. C. (2010) Modeling shifts in the rate and pattern of subthalamopallidal network activity during deep brain stimulation, Journal of Computational Neuroscience, 28(3), 425-441.

Halliday, D. M. and Rosenberg, J. R. (1999) Time and frequency domain analysis of spike train and time series data, Modern Techniques in Neuroscience Research, Springer Berlin Heidelberg, 503-543.

Hammond, C., Bergman, H. and Brown, P. (2007) Pathological synchronization in Parkinson's disease: networks, models and treatments, Trends in Neurosciences, 30(7), 357-364.

Hampson, E. C. G. M., Weiler, R. and Vaney, D. I. (1992) Regulation of gap-junction permeability between A-type horizontal cells in rabbit retina-effects of ph and dopamine, Investigative Ophthalmology, 33(4), 1406-1406.

Hansel, D., Mato, G. and Meunier, C. (1995) Synchrony in excitatory neural networks, Neural computation, 7(2), 307-337.

Hanson, J. E., Smith, Y. and Jaeger, D. (2004) Sodium channels and dendritic spike initiation at excitatory synapses in globus pallidus neurons, The Journal of Neuroscience, 24(2), 329-340.

Hardman, C. D. and Halliday, G. M. (1999) The external globus pallidus in patients with Parkinson's disease and progressive supranuclear palsy, Movement Disorders, 14(4), 626-633.

Hashimoto, K. and Kita, H. (2006) Slow oscillatory activity of rat globus pallidus neurons in vitro, European Journal of Neuroscience, 23(2), 443-453.

Hauber, W. and Lutz, S. (1999) Dopamine $D_{1}$ or $D_{2}$ receptor blockade in the globus pallidus produces akinesia in the rat, Behavioural Brain Research, 106(1-2), 143-150.

Heimer, G., Bar-Gad, I., Goldberg, J. A. and Bergman, H. (2002) Dopamine replacement therapy reverses abnormal synchronization of pallidal neurons in the 1-methyl-4-phenyl-1,2,3,6tetrahydropyridine primate model of parkinsonism, Journal of Neuroscience, 22(18), 7850-7855.

Herzog, R. I., Liu, C., Waxman, S. G. and Cummins, T. R. (2003) Calmodulin binds to the C terminus of sodium channels Nav1. 4 and Nav1. 6 and differentially modulates their functional properties, The Journal of Neuroscience, 23(23), 8261-8270.

Hikosaka, O. (2007) GABAergic output of the basal ganglia, Progress in Brain Research, 160, 209226. 
Holt, A. B. and Netoff, T. I. (2014) Origins and suppression of oscillations in a computational model of Parkinson's disease, Journal of Computational Neuroscience, 37(3), 505-521.

Hoover, B. R. and Marshall, J. F. (2002) Further characterization of preproenkephalin mRNAcontaining cells in the rodent globus pallidus, Neuroscience, 111(1), 111-125.

Horak, F. B. and Anderson, M. E. (1984) Influence of globus pallidus on arm movements in monkeys. I. Effects of kainic acid-induced lesions, Journal of Neurophysiology, 52(2), 290-304.

Hu, X. T., Dong, Y., Zhang, X. F. and White, F. J. (2005) Dopamine D2 receptor-activated Ca2+ signaling modulates voltage-sensitive sodium currents in rat nucleus accumbens neurons, Journal of Neurophysiology, 93(3), 1406-1417.

Ilinsky, I. A., Yi, H. and Kultas-llinsky, K. (1997) Mode of termination of pallidal afferents to the thalamus: a light and electron microscopic study with anterograde tracers and immunocytochemistry in Macaca mulatta, Journal of Comparative Neurology, 386(4), 601-612.

Ingham, C. A., Hood, S. H., Mijnster, M. J., Baldock, R. A. and Arbuthnott, G. W. (1997) Plasticity of striatopallidal terminals following unilateral lesion of the dopaminergic nigrostriatal pathway: a morphological study, Exp Brain Res 116, 39-49.

Jaeger, D. and Kita, H. (2011) Functional connectivity and integrative properties of globus pallidus neurons, Neuroscience, 198, 44-53.

Janssen, M. L., Zwartjes, D. G., Tan, S. K., Vlamings, R., Jahanshahi, A., Heida, T., Hoogland, G., Steinbusch, H. W. M., Visser-Vandewalle, V. and Temel, Y. (2012) Mild dopaminergic lesions are accompanied by robust changes in subthalamic nucleus activity, Neuroscience letters, 508(2), 101-105.

Kammermeier, S., Hamada, I., Devergnas, A., Pittard, D., Smith, Y. and Wichmann, T. (2014) Effects of stimulation of the internal globus pallidus on thalamic activity patterns in parkinsonian monkeys, Society for Neuroscience Annual Meeting, poster 248.12.

Katsumaru, H., Kosaka, T., Heizmann, C. W. and Hama, K. (1988) Gap junctions on GABAergic neurons containing the calcium-binding protein parvalbumin in the rat hippocampus (CA1 region), Experimental Brain Research, 72(2), 363-370.

Kawasaki, A., Hayashi, T., Nakachi, K., Trosko, J. E., Sugihara, K., Kotake, Y. and Ohta, S. (2009) Modulation of connexin 43 in rotenone-induced model of Parkinson's disease, Neuroscience, 160(1), 61-68.

Kita, H., Kosaka, T., and Heizmann, C. W. (1990) Parvalbumin-immunoreactive neurons in the rat neostriatum: a light and electron microscopic study, Brain Research, 536(1), 1-15.

Kita, H. (1994) Parvalbumin-immunopositive neurons in rat globus pallidus: a light and electron microscopic study, Brain Research 657, 31-41.

Kita, H., Nambu, A., Kaneda, K., Tachibana, Y. and Takada, M. (2004) Role of ionotropic glutamatergic and $C_{1} A B A e r g i c$ inputs on the firing activity of neurons in the external pallidum in awake monkeys, Journal of Neurophysiology, 92(5), 3069-3084.

Kita, H., Chiken, S., Tachibana, Y. and Nambu, A. (2006) Origins of GABAA and GABAB receptormediated responses of globus pallidus induced after stimulation of the putamen in the monkey, The Journal of Neuroscience, 26(24), 6554-6562.

Kita, H. (2007) Cilobus pallidus external segment, Progress in Brain Research, 160, 111-133.

Kühn, A. A., Kupsch, A., Schneider, G.-H. and Brown, P. (2006) Reduction in subthalamic 8-35 Hz oscillatory activity correlates with clinical improvement in Parkinson's disease, European Journal of Neuroscience, 23(7), 1956-1960.

Kühn, A. A., Kempf, F., Brücke, C., Doyle, L. G., Martinez-Torres, I., Pogosyan, A., Trottenberg, T., Kupsch, A., Schneider, G.-H., Hariz, M. I., Vandenberghe, W., Nuttin B., and Brown, P. (2008) Highfrequency stimulation of the subthalamic nucleus suppresses oscillatory beta activity in patients 
with Parkinson's disease in parallel with improvement in motor performance, The Journal of Neuroscience, 28(24), 6165-6173.

Kühn, A. A., Tsui, A., Aziz, P., Ray, N., Brücke, C. Kupsch, A., Schneider, G.-H. and Brown, P. (2009) Pathological synchronisation in the subthalamic nucleus of patients with Parkinson's disease relates to both bradykinesia and rigidity, Experimental Neurology, 215(2), 380-387.

Kultas-llinsky, K., Reising, L., Yi, H. and Ilinsky, I. A. (1997) Pallidal afferent territory of the Macaca mulatta thalamus: neuronal and synaptic organization of the VAdc, Journal of Comparative Neurology, 386(4), 573-600.

Kumar, A., Cardanobile, S., Rotter, S. and Aertsen, A. (2011) The role of inhibition in generating and controlling Parkinson's disease oscillations in the basal ganglia, Frontiers in Systems Neuroscience, 5 (86).

Lange, H., Thörner, G., Hopf, A. and Schröder, K. F. (1976) Morphometric studies of the neuropathological changes in choreatic diseases, Journal of the Neurological Sciences, 28(4), 401-425.

Leblois, A., Boraud, T., Meissner, W., Bergman, H. and Hansel, D. (2006) Competition between feedback loops underlies normal and pathological dynamics in the basal ganglia, The Journal of Neuroscience, 26(13), 3567-3583.

Leblois, A., Meissner, W., Bioulac, B., Grross, C. E., Hansel, D. and Boraud, T. (2007) Late emergence of synchronized oscillatory activity in the pallidum during progressive parkinsonism, European Journal of Neuroscience, 26(6), 1701-1713.

Legendy, C. R. and Salcman, M. (1985) Bursts and recurrences of bursts in the spike trains of spontaneously active striate cortex neurons, Journal of Neurophysiology, 53(4), 926-939.

Lewis, T. J. and Rinzel, J. (2003) Dynamics of spiking neurons connected by both inhibitory and electrical coupling, Journal of Computational Neuroscience, 14(3), 283-309.

Li, H., Zhang, Z., Blackburn, M. R., Wang, S. W., Ribelayga, C. P. and O'Brien, J. (2013) Adenosine and dopamine receptors coregulate photoreceptor coupling via gap junction phosphorylation in mouse retina, The Journal of Neuroscience, 33(7), 3135-3150.

Lourens, M. A., Schwab, B. C., Nirody, J. A., Meijer, H. G. and van Gils, S. A. (2015) Exploiting pallidal plasticity for stimulation in Parkinson's disease, Journal of Neural Engineering, 12(2), 026005.

Ma, Y., Hioki, H., Konno, M., Pan, S., Nakamura, H., Nakamura, K. C., Furuta, T., Li, J. and Kaneko, T. (2011) Expression of gap junction protein connexin36 in multiple subtypes of GABAergic neurons in adult rat somatosensory cortex, Cerebral Cortex, 21(11), 2639-2649.

Magill, P. J., Bolam, J. P. and Bevan, M. D. (2001) Dopamine regulates the impact of the cerebral cortex on the subthalamic nucleus-globus pallidus network, Neuroscience, 106(2), 313-330.

Magill, P. J., Pogosyan, A., Sharott, A., Csicsvari, J., Bolam, J. P. and Brown, P. (2006) Changes in functional connectivity within the rat striatopallidal axis during global brain activation in vivo, Journal of Neuroscience, 26(23), 6318-6329.

Mallet, N., Pogosyan, A., Marton, L. F., Bolam, J. P., Brown, P. and Magill, P. J. (2008) Parkinsonian beta oscillations in the external globus pallidus and their relationship with subthalamic nucleus activity, Journal of Neuroscience, 28(52), 14245-14258.

Mallet, N., Micklem, B. R., Henny, P., Brown, M. T., Williams, C., Bolam, J. P., Nakamura, K. C. and Magill, P. J. (2012) Dichotomous organization of the external globus pallidus, Neuron, 74(6), 1075-1086.

Martin, K. A. and Schröder, S. (2016) Phase Locking of Multiple Single Neurons to the Local Field Potential in Cat V1, The Journal of Neuroscience, 36(8), 2494-2502.

Mastro, K. J., Bouchard, R. S., Holt, H. A. and Gittis, A. H. (2014) Transgenic mouse lines subdivide external segment of the globus pallidus (GPe) neurons and reveal distinct $G_{1} P e$ output pathways, The Journal of Neuroscience, 34(6), 2087-2099. 
MATLAB version 7.14. Natick, Massachusetts: The MathWorks Inc., 2012.

Mathai, A., Ma, Y., Paré, J. F., Villalba, R. M., Wichmann, T. and Smith, Y. (2015) Reduced cortical innervation of the subthalamic nucleus in MPTP-treated parkinsonian monkeys, Brain, 138(4), 946-962.

McCairn, K. W. and Turner, R. S. (2009) Deep brain stimulation of the globus pallidus internus in the parkinsonian primate: local entrainment and suppression of low-frequency oscillations, Journal of Neurophysiology, 101(4), 1941-1960.

McFarland, N. R. and Haber, S. N. (2000) Convergent inputs from thalamic motor nuclei and frontal cortical areas to the dorsal striatum in the primate, The Journal of Neuroscience, 20(10), 37983813.

McHahon, D. G., Knapp, A. G. and Dowling, J. E. (1989) Horizontal cell gap junctions: single-channel conductance and modulation by dopamine, Proceedings of the National Academy of Sciences, 86(19), 7639-7643.

Meijer, H. G. E., Krupa, M., Cagnan, H., Lourens, M. A. J., Heida, T., Martens, H. C. F., Bour, L. and van Cils, S. A. (2011) From Parkinsonian thalamic activity to restoring thalamic relay using deep brain stimulation: new insights from computational modeling, Journal of Neural Engineering, 8(6), 066005 .

Mercer, J. N., Chan, C. S., Tkatch, T., Held, J. and Surmeier, D. J. (2007) Nav1. 6 sodium channels are critical to pacemaking and fast spiking in globus pallidus neurons, The Journal of Neuroscience, 27(49), 13552-13566.

Middleton, F. A. and Strick, P. L. (2000) Basal ganglia and cerebellar loops: Motor and cognitive circuits, Brain Research Reviews, 31(2), 236-250.

Miguelez, C., Morin, S., Martinez, A., Goillandeau, M., Bezard, E., Bioulac, B. and Baufreton, J. (2012) Altered pallido-pallidal synaptic transmission leads to aberrant firing of globus pallidus neurons in a rat model of Parkinson's disease, Journal of Physiology, 590(22), 5861-5875.

Milardi, D., Gaeta, M., Marino, S., Arrigo, A., Vaccarino, G., Mormina, E., Rizzo, G., Milazzo C., Finocchio G., Baglieri A., Anastasi G. and Quartarone, A. (2015) Basal ganglia network by constrained spherical deconvolution: A possible cortico-pallidal pathway?, Movement Disorders, 30(3), $342-$ 349.

Mink, J. W. and Thach, W. T. (1991) Basal ganglia motor control. II. Late pallidal timing relative to movement onset and inconsistent pallidal coding of movement parameters, Journal of Neurophysiology, 65(2), 301-329.

Montgomery, E. B. Jr (2007) Basal ganglia physiology and pathophysiology: A reappraisal, Parkinsonism \& Related Disorders, 13(8), 455-465.

Moore, H. and Cirace, A. A. (2002) A role for electrotonic coupling in the striatum in the expression of dopamine receptor-mediated stereotypies, Neuropsychopharmacology, 27(6), 980-992.

Morris, G., Nevet, A. and Bergman, H. (2003) Anatomical funneling, sparse connectivity and redundancy reduction in the neural networks of the basal ganglia, Journal of Physiology-Paris, 97(4), 581-589.

Naito, A. and Kita, H. (1994) The cortico-pallidal projection in the rat: an anterograde tracing study with biotinylated dextran amine, Brain Research, 653(1), 251-257.

Nambu, A., Yoshida, S. and Jinnai, K. (1991) Movement-related activity of thalamic neurons with input from the globus pallidus and projection to the motor cortex in the monkey, Experimental brain research, 84(2), 279-284.

Nambu, A. and Llinas, R. (1994) Electrophysiology of globus pallidus neurons in vitro, Journal of neurophysiology, 72(3), 1127-1139. 
Nambu, A. and Llinas, R. (1997) Morphology of globus pallidus neurons: Its correlation with electrophysiology in guinea pig brain slices, Journal of Comparative Neurology, 377(1), 85-94

Nambu, A. (2004) A new dynamic model of the cortico-basal ganglia loop, Progress in Brain Research, 143, 461-466.

Nambu, A. (2007) Globus pallidus internal segment, Progress in Brain Research, 160, 135-150.

Nemani, V. M. and Binder, D. K. (2005) Emerging role of gap junctions in epilepsy, Histology and Histopathology, 20, 253-259.

Nini, A., Feingold, A., Slovin, H., and Bergman, H. (1995) Neurons in the globus pallidus do not show correlated activity in the normal monkey, but phase-locked oscillations appear in the MPTP model of parkinsonism,Journal of Neurophysiology, 74(4), 1800-1805.

Nobrega-Pereira, S., Gelman, D., Bartolini, G., Pla, R., Pierani, A. and Marín, O. (2010) Origin and molecular specification of globus pallidus neurons, The Journal of Neuroscience, 30(8), 2824-2834.

Phookan, S., Sutton, A. C., Walling, I., Smith, A., O'Connor, K. A., Campbell, J. C., Calosa, M., Yua, W., Pilitsisa, J. G., Brotchie, J.M. and Shin, D. S. (2015) Gap junction blockers attenuate beta oscillations and improve forelimb function in hemiparkinsonian rats, Experimental Neurology, 265, 160-170.

Poolos N. P. (2012) Hyperpolarization-Activated Cyclic Nucleotide-Gated (HCN) lon Channelopathy in Epilepsy, Jasper's Basic Mechanisms of the Epilepsies, 4th edition.

Obeso, J. A., Rodriguez-Oroz, M. C., Rodriguez, M., Lanciego, J. L., Artieda, J., Cıonzalo, N. and Olanow, C. W. (2000) Pathophysiology of the basal ganglia in Parkinson's disease, Trends in Neurosciences, 23, S8-S19.

Onn, S. P. and Grace, A. A. (1999) Alterations in electrophysiological activity and dye coupling of striatal spiny and aspiny neurons in dopamine-denervated rat striatum recorded in vivo, Synapse, 33(1), 1-15.

Oorschot, D. E. (1996) Total number of neurons in the neostriatal, pallidal, subthalamic, and substantia nigral nuclei of the rat basal ganglia: a stereological study using the cavalieri and optical disector methods, Journal of Comparative Neurology, 366(4), 580-599.

Palacios-Prado, N., Hoge, G., Marandykina, A. et al. (2013) Intracellular magnesium-dependent modulation of gap junction channels formed by neuronal connexin36, The Journal of Neuroscience, 33(11), 4741-4753.

Parent, M. and Parent, A. (2004) The pallidofugal motor fiber system in primates, Parkinsonism $\varepsilon_{1}$ Related Disorders, 10(4), 203-211.

Pasquereau, B., DeLong, M. R. and Turner, R. S. (2015) Primary motor cortex of the parkinsonian monkey: altered encoding of active movement, Brain, awv312.

Pavlides, A., Hogan, S. J. and Bogacz, R. (2015) Computational Models Describing Possible Mechanisms for Generation of Excessive Beta Oscillations in Parkinson's Disease, PLoS Computational Biology, 11(12), e1004609.

Percival, D. B. and Walden, A. T. (1993) Spectral analysis for physical applications. Cambridge University Press.

Person, A. L. and Perkel, D. J. (2005) Unitary IPSPs drive precise thalamic spiking in a circuit required for learning, Neuron, 46(1), 129-140.

Person, A. L. and Perkel, D. J. (2007) Pallidal neuron activity increases during sensory relay through thalamus in a songbird circuit essential for learning, The Journal of Neuroscience, 27(32), 86878698.

Percheron, G., François, C., Parent, A., Sadikot, A. F., Fenelon, G. and Yelnik, J. (1991) The primate central complex as one of the basal ganglia, The Basal Ganglia III, 39, 177-186. 
Perkel, D. H., Gierstein, G. L. and Moore, G. P. (1967) Neuronal spike trains and stochastic point processes: II. Simultaneous spike trains, Biophysical Journal, 7(4), 419-440.

Pessiglione, M., Guehl, D., Rolland, A. S., François, C., Hirsch, E. C., Féger, J. and Tremblay, L. (2005) Thalamic neuronal activity in dopamine-depleted primates: evidence for a loss of functional segregation within basal ganglia circuits, The Journal of Neuroscience, 25(6), 1523-1531.

Pfeuty, B. (2005) The combined effects of inhibitory and electrical synapses in synchrony, Neural Computation, 17, 633-670.

Pirini, M., Rocchi, L., Sensi, M. and Chiari, L. (2009) A computational modelling approach to investigate different targets in deep brain stimulation for Parkinson's disease, Journal of Computational Neuroscience, 26(1), 91-107.

Plenz, D. and Kital, S. T. (1999) A basal ganglia pacemaker formed by the subthalamic nucleus and external globus pallidus, Nature, 400, 677-682.

Pogosyan, A., Gaynor, L. D., Eusebio, A. and Brown, P. (2009) Boosting cortical activity at beta-band frequencies slows movement in humans, Current Biology, 19(19), 1637-1641.

Priestley, M. B. (1981) Spectral analysis and time series, Volume 1. Academic press.

Quiroga-Varela, A., Walters, J. A., Brazhnik, E., Marin, C. and Obeso, J. A. (2013) What basal ganglia changes underlie the parkinsonian state? The significance of neuronal oscillatory activity, Neurobiology of Disease, doi: 10.1016/j.nbd.2013.05.010.

Rajput, A. H., Sitte, H. H., Rajput, A., Fenton, M. E., Pifl, C. and Hornykiewicz, O. (2008) Globus pallidus dopamine and Parkinson motor subtypes, Neurology, 70(16), 1403-1410.

Ramanathan, S., Tkatch, T., Atherton, J. F., Wilson, C. J. and Bevan, M. D. (2008) D2-like dopamine receptors modulate SKCa channel function in subthalamic nucleus neurons through inhibition of Cav2. 2 channels, Journal of Neurophysiology, 99(2), 442-459.

Rasband, W.S., ImageJ, U. S. National Institutes of Health, Bethesda, Maryland, USA, http://imagej.nih.gov/ij/, 1997-2012.

Rascol, O., Sabatini, U., Chollet, F., Celsis, P., Montastruc, J. L., Marc-Vergnes, J. P. and Rascol, A. (1992) Supplementary and primary sensory motor area activity in Parkinson's disease: regional cerebral blood flow changes during finger movements and effects of apomorphine, Archives of Neurology, 49(2), 144-148.

Rash, J. E., Staines, W. A., Yasumura, T., Patel, D., Furman, C. S., Stelmack, G. L. and Nagy, J. I. (2000) Immunogold evidence that neuronal gap junctions in adult rat brain and spinal cord contain connexin-36 but not connexin-32 or connexin-43, Proceedings of the National Academy of Sciences, 97(13), 7573-7578.

Raz, A., Vaadia, E. and Bergman, H. (2000) Firing patterns and correlations of spontaneous discharge of pallidal neurons in the normal and the tremulous 1-methyl-4-phenyl-1, 2, 3, 6tetrahydropyridine vervet model of parkinsonism, The Journal of Neuroscience, 20(22), 8559-8571.

Reitsma, P., Doiron, B. and Rubin, J. E. (2011) Correlation transfer from basal ganglia to thalamus in Parkinson's disease, Frontiers in Computational Neuroscience, 5.

Riehle, A., Grün, S., Diesmann, M. and Aertsen, A. (1997) Spike synchronization and rate modulation differentially involved in motor cortical function, Science, 278(5345), 1950-1953.

Rubin, J. E. and Terman, D. (2004) High frequency stimulation of the subthalamic nucleus eliminates pathological thalamic rhythmicity in a computational model, Journal of Computational Neuroscience, 16(3), 211-235.

Rubin, J. E., Mclntyre, C. C., Turner, R. S. and Wichmann, T. (2012) Basal ganglia activity patterns in parkinsonism and computational modeling of their downstream effects, European Journal of Neuroscience, 36(2), 2213-2228. 
Sadek, A. R., Magill, P. J., and Bolam, J. P. (2005) Local connectivity between neurons of the rat globus pallidus, The basal ganglia VIII , 56, 611-619.

Sadek, A. R., Magill, P. J. and Bolam, J. P. (2007) A single-cell analysis of intrinsic connectivity in the rat globus pallidus, Journal of Neuroscience, 27(24), 6352-6362.

Schultheiss, N. W., Edgerton, J. R. and Jaeger, D. (2010) Phase response curve analysis of a full morphological globus pallidus neuron model reveals distinct perisomatic and dendritic modes of synaptic integration, The Journal of Neuroscience, 30(7), 2767-2782.

Schultheiss, N. W., Edgerton, J. R. and Jaeger, D. (2012) Robustness, variability, phase dependence, and longevity of individual synaptic input effects on spike timing during fluctuating synaptic backgrounds: a modeling study of globus pallidus neuron phase response properties, Neuroscience, 219, 92-110.

Schwab, B. C., Heida, T., Zhao, Y., Marani, E., van Gils, S. A. and van Wezel, R. J. A. (2013a) Synchrony in Parkinson's disease: Importance of intrinsic properties of the external pallidal segment, Frontiers in Systems Neuroscience, 7(60).

Schwab, B. C., Seemann, G., Lasher, R. A., Torres, N. S., Wulfers, E. M., Arp, M., Carruth, E. D., Bridge, J. H. B. and Sachse, F. B. (2013b) Quantitative analysis of cardiac tissue including fibroblasts using three-dimensional confocal microscopy and image reconstruction: towards a basis for electrophysiological modeling, IEEE Transactions on Medical Imaging, 32(5), 862-872.

Schwab, B. C., Heida, T., Zhao, Y., van Cils, S. A. and van Wezel, R. J. A. (2014) Pallidal gap junctions - Triggers of synchrony in Parkinson's disease?, Movement Disorders, 29(12), 1486-1494.

Schwab, B. C., van Wezel, R. J. A. and van Cils, S. A. (2016) Sparse pallidal connectivity shapes synchrony in a network model of the basal ganglia, submitted.

Sharott, A., Magill, P. J., Harnack, D., Kupsch, A., Meissner, W. and Brown, P. (2005) Dopamine depletion increases the power and coherence of $\beta$-oscillations in the cerebral cortex and subthalamic nucleus of the awake rat, European Journal of Neuroscience, 21(5), 1413-1422.

Shink, E. and Smith, Y. (1995) Differential synaptic innervation of neurons in the internal and external segments of the globus pallidus by the CABA- and glutamate-containing terminals in the squirrel monkey, Journal of Comparative Neurology, 358(1), 119-141.

Sims, R. E., Woodhall, G. L., Wilson, C. L. and Stanford, I. M. (2008) Functional characterization of $\mathrm{G}_{1} A B A$ ergic pallidopallidal and striatopallidal synapses in the rat globus pallidus in vitro, European Journal of Neuroscience, 28(12), 2401-2408.

Smith, Y., Bevan, M. D., Shink, E. and Bolam, J. P. (1998) Microcircuitry of the direct and indirect pathways of the basal ganglia, Neuroscience, 86(2), 353.

Smith, Y., Raju, D. V., Pare, J. F. and Sidibe, M. (2004) The thalamostriatal system: a highly specific network of the basal ganglia circuitry, Trends in Neurosciences, 27(9), 520-527.

Smith, Y. and Wichmann, T. (2015) The cortico-pallidal projection: An additional route for cortical regulation of the basal ganglia circuitry, Movement Disorders, 30(3), 293-295.

Soehl, G., Maxeiner, S. and Willecke, K. (2005) Expression and functions of neuronal gap junctions, Nature Reviews Neuroscience, 6(3), 191-200.

Spray, D.C., Harris, A.L. and Bennett, M.V. (1981) Gap Junctional Conductance Is Simple and Sensitive Function of intracellular $\mathrm{pH}$, Science, 211, 13.

Stanford, I. M. and Cooper, A. J. (1999) Presynaptic $\mu$ and $\delta$ opioid receptor modulation of GABAA IPSCs in the rat globus pallidus in vitro, The Journal of Neuroscience, 19(12), 4796-4803.

Stein, E. and Bar-Gad, I. (2013) Beta oscillations in the cortico-basal ganglia loop during parkinsonism, Experimental Neurology, 245, 52-59. 
Sung, J. Y., Lee, H. J., Jeong, E. I., Oh, Y., Park, J., Kang, K. S. and Chung, K. C. (2007) $\alpha$-Synuclein overexpression reduces gap junctional intercellular communication in dopaminergic neuroblastoma cells, Neuroscience Letters, 416(3), 289-293.

Tachibana, Y., Iwamuro, H., Kita, H., Takada, M. and Nambu, A. (2011) Subthalamo-pallidal interactions underlying parkinsonian neuronal oscillations in the primate basal ganglia, European Journal of Neuroscience, 34(9), 1470-1484.

Tamás, G., Buhl, E. H., Lörincz, A. and Somogyi, P. (2000) Proximally targeted GABAergic synapses and gap junctions synchronize cortical interneurons, Nature Neuroscience, 3(4), 366-371.

Terman, D., Rubin, J. E., Yew, A. C., and Wilson, C. Y. (2002) Activity patterns in a model for the subthalamopallidal network of the basal ganglia, Journal of Neuroscience, 22(7), 2963-2976.

Terman, D., Rubin, J. E. and Diekman, C. O. (2013) Irregular activity arises as a natural consequence of synaptic inhibition, Chaos: An Interdisciplinary Journal of Nonlinear Science, 23(4), 046110.

Traub, R. D., Bibbig, A., Fisahn, A., LeBeau, F. E., Whittington, M. A. and Buhl, E. H. (2000) A model of gamma-frequency network oscillations induced in the rat $C A 3$ region by carbachol in vitro, European Journal of Neuroscience, 12(11), 4093-4106.

Traub, R. D. and Whittington, M. A. (2010) Cortical oscillations in health and disease, Oxford University Press.

Turner, R. S. and Anderson, M. E. (1997) Pallidal discharge related to the kinematics of reaching movements in two dimensions, Journal of Neurophysiology, 77(3), 1051-1074.

Turner, R. S., Desmurget, M., Grethe, J., Crutcher, M. D. and Girafton, S. T. (2003) Motor subcircuits mediating the control of movement extent and speed, Journal of Neurophysiology, 90(6), 39583966.

Vandecasteele, M., Cllowinski, J. and Venance, L. (2005) Electrical synapses between dopaminergic neurons of the substantia nigra pars compacta, The Journal of Neuroscience, 25(2), 291-298.

Vandecasteele, M., Deniau, J. M., Gilowinski, J. and Venance, L. (2007) Electrical synapses in basal ganglia, Reviews in the Neurosciences, 18(1), 15-36.

Van Vreeswijk, C., Abbott, L. F. and Ermentrout, G. B. (1994) When inhibition not excitation synchronizes neural firing, Journal of Computational Neuroscience, 1(4), 313-321.

Vervaeke, K., Lorincz, A., Clleeson, P., Farinella, M., Nusser, Z. and Silver, R. A. (2010) Rapid desynchronization of an electrically coupled interneuron network with sparse excitatory synaptic input, Neuron, 67(3), 435-451.

Vitek, J. L., Hashimoto, T., Peoples, J., DeLong, M. R. and Bakay, R. A. (2004) Acute stimulation in the external segment of the globus pallidus improves parkinsonian motor signs, Movement Disorders, 19(8), 907-915.

Wang, Y., Denisova, J. V., Kang, K. S., Fontes, J. D., Zhu, B. T. and Belousov, A. B. (2010) Neuronal gap junctions are required for NMDA receptor-mediated excitotoxicity: Implications in ischemic stroke, Journal of Neurophysiology, 104(6), 3551-3556.

Weinberger, M. and Dostrovsky, J. O. (2011) A basis for the pathological oscillations in basal ganglia: the crucial role of dopamine, Neuroreport, 22(4), 151.

Wegiel, J., Flory, M., Kuchna, I., Nowicki, K., Ma, S., Imaki, H., Wegiel, J., Cohen, I. L., London, E., Wisniewski, T., and Brown, W. (2014) Stereological study of the neuronal number and volume of 38 brain subdivisions of subjects diagnosed with autism reveals significant alterations restricted to the striatum, amygdala and cerebellum, Acta Neuropathologica Communications, 2, 141.

Wichmann, T. (2006) Basal ganglia discharge abnormalities in Parkinson's disease, Parkinson's Disease and Related Disorders, 21-25. 
Wichmann, T. and Soares, J. (2006) Neuronal firing before and after burst discharges in the monkey basal ganglia is predictably patterned in the normal state and altered in parkinsonism, Journal of Neurophysiology, 95(4), 2120-2133.

Wichmann, T., DeLong, M. R., Guridi, J. and Obeso, J. A. (2011) Milestones in research on the pathophysiology of Parkinson's disease, Movement Disorders, 26(6), 1032-1041.

Wichmann, T. and Smith, Y. (2013) Extrastriatal plasticity in parkinsonism, Basal Ganglia, 3(1), 5-8.

Wilson, C. J., Bryce Beverlin, I. I. and Netoff, T. (2011) Chaotic desynchronization as the therapeutic mechanism of deep brain stimulation, Frontiers in Systems Neuroscience, 5.

Wilson, C. J. (2013) Active Decorrelation in the Basal Ganglia, Neuroscience.

Witkovsky, P., Owen, W. G. and Woodworth, M. (1983) Gap junctions among the perikarya, dendrites, and axon terminals of the luminosity-type horizontal cell of the turtle retina, Journal of Comparative Neurology, 216(4), 359-368.

Woodward Hopf, F., Seif, T., Mohamedi, M. L., Chen, B. T. and Bonci, A. (2010) The small-conductance calcium-activated potassium channel is a key modulator of firing and long-term depression in the dorsal striatum, European Journal of Neuroscience, 31(11), 1946-1959.

Xu, J., Kang, N., Jiang, L., Nedergaard, M. and Kang, J. (2005) Activity-dependent long-term potentiation of intrinsic excitability in hippocampal CA1 pyramidal neurons, The Journal of Neuroscience, 25(7), 1750-1760.

Yamawaki, N., Stanford, I. M., Hall, S. D. and Woodhall, G. L. (2008) Pharmacologically induced and stimulus evoked rhythmic neuronal oscillatory activity in the primary motor cortex in vitro, Neuroscience, 151(2), 386-395.

Yelnik, J. (2002) Functional anatomy of the basal ganglia, Movement Disorders, 17, S15-S21.

Zimnik, A. J., Nora, G. J., Desmurget, M. and Turner, R. S. (2015) Movement-Related Discharge in the Macaque Gilobus Pallidus during High-Frequency Stimulation of the Subthalamic Nucleus, The Journal of Neuroscience, 35(9), 3978-3989.

Zimnik, A. J. and Turner, R. S. (2015) Basal ganglia output is not the determinant of movementrelated activity in the pallidal-recipient thalamus, Society for Neuroscience Annual Meeting, poster 2015-S-17042-SfN. 



\section{List of Abbreviations}

CCF

CCCF

pCCF

Cx

DBS

EMG

EP

FSI

G.P

G.Pe

GPi

$\mathrm{HCN}$

L-Dopa

LFP

MPTP

mRNA

MSN

M1

$\mathrm{NaF}$

PCA

$P D$

PRC

$P V$

SK

SCP

SEM

$\mathrm{SNc}$

$\mathrm{SNr}$ cross-correlation function

corrected cross-correlation function

predicted cross-correlation function

connexin

deep brain stimulation

electromyography

endopenduncular nucleus

fast-spiking interneuron

globus pallidus

external part of the globus pallidus

internal part of the globus pallidus

hyperpolarization-activated cyclic nucleotide-gated

L-3,4-dihydroxyphenylalanine

local field potential

1-Methyl-4-Phenyl-1,2,3,6-Tetrahydropyridine

messenger ribonucleic acid

medium spiny neuron

here: primary motor cortex

fast, transient, voltage-dependent sodium

principal component analysis

Parkinson's disease

phase response curve

parvalbumin

small conductance calcium-activated potassium

superior cerebellar peduncle

standard error of the mean

substantia nigra pars compacta

substantia nigra pars reticulata 
STA spike triggered average

STN subthalamic nucleus

tACS transcranial alternating current stimulation

VLa ventrolateral anterior nucleus of thalamus

6-OHDA 6-hydroxydopamine 


\section{Summary}

Patients with Parkinson's disease (PD) typically suffer severely from different types of symptoms. Motor symptoms, restricting the patients' ability to perform controlled movements in daily life, are of special clinical interest and have been related to neural activity in the basal ganglia. Low-frequency oscillations, bursting (short periods of high-frequency spiking) and especially synchrony of basal ganglia activity emerge after dopamine depletion that follows cell death in the substantia nigra pars compacta. These changes are all believed to contribute to motor symptoms. In this thesis, we described a possible mechanism for the emergence of synchrony in the basal ganglia of PD patients based on gap junctions in the external part of the globus pallidus (GPe), using a combination of experimental and computational methods.

Firstly, we argued why the C.Pe is our focus of attention (Chapter 2). During the last years, several studies have demonstrated that the GPe projects to all basal ganglia nuclei, putting itself in a hub position. Forming feedback loops with neighboring nuclei, for example the subthalamic nucleus or the striatum, the GPe can powerfully orchestrate activity in the basal ganglia. In particular, adaptions of neural properties or connectivity of C.Pe neurons might evoke strong changes in the dynamics of the whole basal ganglia. As shifts in synchrony after dopamine depletion are most prominent in $\mathrm{G}_{1} \mathrm{Pe}$, it can be speculated that crucial pathological processes in PD occur in this nucleus.

In Chapter 3, we investigated the hypothesis that gap junctions could be present between neurons of G.Pe. As a first indication of neural gap junction coupling, we looked for the protein Connexin-36 with immunohistochemistry and confocal imaging. $\mathrm{C}_{1} A B$ Aergic neurons in $\mathrm{C}_{1} \mathrm{Pe}$ and also the internal part of the globus pallidus indeed showed Connexin-36, while the protein was absent in the subthalamic nucleus. We also detected a slight increase in the number of Connexin-36 spots in PD patients compared to control subjects. However, we do not know which fraction of the detected Connexin-36 spots actually indicates functional gap junctions, including the 
possibility of no functional gap junction coupling at all. In a small, existing network model of the basal ganglia, we demonstrated that pallidal gap junctions could have a high influence on synchrony in the basal ganglia. Thus, we suggested that gap junctions may exist in C.Pe and could influence subcortical synchrony.

We elaborate a more detailed basal ganglia network model in Chapter 4. Connection architectures and strengths were adapted to experimental data, with a focus on connectivity between C.Pe neurons. We varied the strength of both pallidal inhibition and gap junctions. Whereas inhibition desynchronized the network, including the neighboring nuclei subthalamic nucleus and internal part of the globus pallidus, gap junctions had a desynchronizing effect only at low strengths. At higher strengths, gap junctions synchronized the network. In particular, gap junctions increased beta synchrony. The effect was present also in a smaller model and occurred in two different $\mathrm{C}_{1} \mathrm{Pe}$ neuron models. Also the concrete connection architecture had only a minor influence on synchrony. We concluded that intrapallidal connectivity is of special importance for synchrony of basal ganglia activity, even if those connections are very sparse.

Lastly, in Chapter 5, we investigated correlations between basal ganglia output and thalamic input activity during movements. These correlations are of particular interest as they provide insights into the physiological role of basal ganglia activity, and how the basal ganglia exert their influence on thalamus. While cell-to-cell interactions were very low even during movement, we found strong correlations between local field potentials in the two structures. The high local field potential correlations could only scarcely be explained by a common linear influence of cortex. When combining correlations of many neuron pairs, a slight but significant coherence in the beta band could be detected. Conclusively, it could be that information transmission from the basal ganglia to thalamus is possible only via the coordinated involvement of many neurons, as reflected in local field potentials. In pathological situations such as PD, this coordination might be hampered.

Many open questions remain. Most importantly, we did not provide evidence for the functionality of pallidal gap junctions, requiring extensive paired recordings in a sparsely coupled network. Nevertheless, given the absence of a functional proof, experiments can be performed to test the potential impact of pallidal gap junctions. For example, Connexin-36 knock-out mice are predicted to show less synchrony after administration of 6-hydroxydopamine than wilde type mice. A better description of basal ganglia anatomy and physiology can help to understand information process- 
ing in subcortical structures, and may finally lead to improved therapies of basal ganglia disorders such as Parkinson's disease. 



\section{Samenvatting}

Patiënten met de ziekte van Parkinson lijden vaak ernstig aan verschillende symptomen. Motorische klachten maken het voor de patiënten moeilijk om gecontroleerde bewegingen uit te voeren. Deze klachten zijn daarom klinisch van bijzonder belang en worden gerelateerd aan neurale activiteit in de basale ganglia. Laag frequente oscillaties, bursts (korte periodes van hoog frequente activiteit) en vooral synchronisatie in de basale ganglia ontstaan nadat dopamine verdwijnt door celdood in de substantia nigra pars compacta. Al deze veranderingen zullen waarschijnlijk bijdragen aan de motorische klachten. In dit proefschrift beschrijven we een mogelijk mechanisme dat het optreden van synchronisatie in de basale ganglia van patiënten met de ziekte van Parkinson kan verklaren. Het mechanisme is gebaseerd op gap junctions in het externe gedeelte van de globus pallidus ( $\mathrm{C}_{1} \mathrm{Pe}$ ) en we maken gebruik van zowel experimentele als ook computationele methodes.

In het begin van dit proefschrift wordt uitgelegd waarom we ons op de GPe concentreren (hoofdstuk 2). Verschillende studies hebben in de laatste jaren laten zien dat vanaf de $C_{1} P e$ projecties naar alle kernen in de basale ganglia bestaan. Daarom zit de GPe in een soort centrale hub positie. Omdat de C.Pe feedback loops vormt met andere kernen, zoals bijvoorbeeld met de subthalamische kern of het striatum, is hij bij uitstek in staat om activiteit in de basale ganglia te regelen. Vooral aanpassingen in de cellulaire eigenschappen van G.Pe cellen of in hun verbindingen kunnen tot sterke veranderingen in de dynamica van de basale ganglia leiden. Aangezien de verschuiving in synchronisatie na afbraak van dopamine het hoogst is in $\mathrm{C}_{1} \mathrm{Pe}$, speculeren we dat cruciale pathologische processen bij de ziekte van Parkinson in deze kern plaatsvinden.

In hoofdstuk 3 hebben we de hypothese onderzocht dat er gap junctions aanwezig zijn tussen $C_{1} P e$ neuronen. Als een eerste aanwijzing voor gap junction koppeling hebben we met immunohistochemie en confocale microscopie gekeken naar de proteine connexine-36. We hebben connexine-36 op GABA neuronen gevonden in de GPe 
en in het interne gedeelte van de globus pallidus, maar niet in de subthalamische kern. Verder hebben we ook een toename van connexine-36 vlekjes bij patiënten met de ziekte van Parkinson vastgesteld in vergelijking met proefpersonen zonder neurologische schade. Echter weten we niet hoeveel van deze connexine-36 vlekjes inderdaad functionele gap junctions vormen en we kunnen zelfs niet uitsluiten dat er helemaal geen functionele koppelingen zijn. In een klein, bestaand netwerk van de basale ganglia hebben we laten zien dat gap junctions in de globus pallidus een duidelijke invloed op synchronisatie kunnen hebben. Daarom hebben we de hypothese geponeerd dat gap junctions in de GPe bestaan en een belangrijke rol spelen bij het niveau van synchronisatie in subcorticale structuren.

Het netwerk model van de basale ganglia is in hoofstuk 4 uitgebouwd. De architectuur en sterkte van de connecties zijn aangepast aan experimentele data, met speciale aandacht voor de verbindingen tussen GPe neuronen. We hebben de sterkte van zowel de inhibitie als het aantal gap junctions in de C.Pe gevarieerd. Hoewel inhibitie het netwerk, inclusief de subthalamische kern en het interne gedeelte van de globus pallidus, kan desynchroniseren, kunnen gap junctions dit alleen doen als de geleidbaarheid laag is. Hogere gap junction geleidbaarheid leidt in dit model tot synchronisatie van het hele netwerk. Deze synchronisatie is in het bijzonder hoger in de beta band in vergelijking met andere frequentie banden. We hebben dit ook gevonden in een klein model met minder cellen, en we hebben ook verschillende modellen voor de G.Pe neuronen gebruikt. De speciale architectuur van verbindingen was minder belangrijk voor de mate van synchronisatie. We hebben geconcludeerd dat connecties in de GPe cruciaal zijn voor het optreden van synchrone activiteit in de basale ganglia, zelfs als het aantal verbindingen per neuron heel klein is.

Tenslotte, in hoofdstuk 5, hebben we correlaties tussen output activiteit van de basale ganglia en input activiteit in de thalamus tijdens bewegingen bestudeerd. Deze correlaties zijn van bijzonder belang, omdat we hiermee een idee krijgen van de fysiologische betekenis van de activiteit in de basale ganglia. Hiervoor hebben we bekeken hoe activiteit in de basale ganglia thalamische activiteit beïnloedt. Hoewel interacties tussen aparte neuronen in de basale ganglia en thalamus bijna nooit significante correlaties lieten zien, waren de correlaties tussen cle lokale veldpotentialen in de twee structuren in het algemeen heel hoog. De correlaties tussen de lokale veldpotentialen konden alleen in heel beperkte mate door een gelijke, lineaire input van cortex worden verklaard. Verder hebben we een geringe, maar significante correlatie in de beta band tussen de gemiddelde activiteit van aparte neuronen in de twee structuren gevonden. We hebben daarom voorgesteld dat transmissie van informatie van de basale ganglia naar de thalamus door de coöperatie van een groot 
aantal aan neuronen zou kunnen gebeuren, wat ook in de lokale veldpotentialen terug te vinden is. In pathologische situaties, zoals bij de ziekte van Parkinson, zou deze coöperatie belemmerd kunnen zijn.

Veel vragen blijven onbeantwoord. De belangrijkst is of, en in welke mate, de gap junctions in het pallidum functioneel zijn. Om dit te bepalen zouden omvangrijke gepaarde afleidingen van GPe neuronen nodig zijn. Desalniettemin is het denkbaar om naar de mogelijke invloed van gap junctions te kijken, ook zonder een bewijs voor functionele koppeling. Bijvoorbeeld voorspellen we dat connexine-36 knock-out muizen na injectie van 6-hydroxydopamine minder beta synchronisatie laten zien dan wilde-type muizen. Een verbeterde beschrijving van anatomie en fysiologie van de basale ganglia zou ons kunnen helpen om informatie verwerking in subcorticale structuren te begrijpen en zou uiteindelijk tot betere therapieën van de ziekte van Parkinson kunnen leiden. 



\section{Zusammenfassung}

Patienten mit der Parkinson-Krankheit leiden typischerweise unter verschiedenen Symptomen. Insbesondere motorische Symptome beeinträchtigen den Patienten stark. Sie machen es im Alltag schwierig, kontrollierte Bewegungen auszuführen, und sind daher von hohem klinischen Belang. Zudem wurde zwischen diesen motorischen Symptomen und neuronaler Aktivität in den Basalganglien eine Korrelation festgestellt. Sowohl niedrigfrequente Oszillationen als auch Bursts (kurze Perioden hochfrequenter Aktivität) und vor allem Synchronität der Aktivität von Neuronen in den Basalganglien entstehen nach Abbau des Neurotransmitters Dopamin. Der Abfall des Dopaminspiegels ist bedingt durch einen neuronalen Zelltod in der Substantia Nigra Pars Compacta. Die beschriebenen Veränderungen in der neuronalen Aktivität scheinen alle zu den motorischen Symptomen beizutragen. In der vorliegenden Dissertation wird ein möglicher Mechanismus beschrieben, der das Auftreten von Synchronität in den Basalganglien von Patienten mit der Parkinson-Krankheit erklären kann. Der Mechanismus basiert auf Gap Junctions im externen Teil des Gilobus Pallidus (GPe). Um den Mechanismus zu untersuchen, verwenden wir eine Kombination von experimentellen und rechnergestützten Methoden.

Zunächst erklären wir, warum wir uns auf den C,Pe konzentrieren (Kapitel 2). In den letzten Jahren haben mehrere Studien gezeigt, dass Synapsen des C.Pe zu allen anderen Kernen der Basalganglien projizieren, und der GiPe sich damit in der Positrons eines zentralen Drehkreuzes befindet. Indem er Feedback-Schleifen mit anderen Kernen formt, zum Beispiel mit dem Subthalamischen Kern oder dem Striatum, hat der GPe besonders ausgeprägte Möglichkeiten, neuronale Aktivität in den gesamten Basalganglien zu beeinflussen. Vor allem Adaptionen der zellulären Eigenschaften oder der Verbindungen innerhalb des GPes könnten deutliche Veränderungen in der Basalganglien-Dynamik hervorrufen. Da der Antieg in Synchronität nach Dopamin-Abbau im GPe am stärksten ausgeprägt ist, spekulieren wir, dass bei der Parkinson-Krankheit entscheidende pathologische Prozesse in diesem Kern 
stattfinden.

In Kapitel 3 untersuchen wir die Hypothese, dass Gap Junctions zwischen GPe Neuronen bestehen. Als einen ersten Anhaltspunkt für Gap Junction Kopplung beschreiben wir das Auftreten von Connexin-36 mit Hilfe von Immunohistochemie und Konfokalmiktroskopie. GABAerge Neuronen im G1Pe sowie im internen Teil des Cilobus Pallidus zeigten deutlich Connexin-36 auf Zellmembranen, während wir im Subthalamischen Kern kein Connexin-36 fanden. Patienten mit der Parkinson-Krankheit besaßen im Mittel etwas mehr Connexin-36 Punkte im Pallidum als Vergleichspersonen ohne neurologische Auffälligkeiten. Es ist jedoch nicht bekannt, wie viele dieser Connexin-36 Punkte tatsächlich auf funktionelle Gap Junctions hindeuten. Dies schließt die Möglichkeit mit ein, dass überhaupt keine funktionellen Crap Junctions bestehen. In einem kleinen, existierenden Netzwerk-Modell der Basalganglien demonstrieren wir, dass Gap Junctions im Pallidum, sofern vorhanden und funktionell, einen hohen Einfluss auf neuronale Synchronität haben können. Wir schlagen daher vor, dass Gap Junctions im G.Pe existieren und subkortikale Synchronität entscheidend beeinflussen können.

Das Netwerk-Modell aus Kapitel 3 wird in Kapitel 4 ausgebaut. Die Architektur und Stärke der synaptischen Verbindungen wurden an experimentelle Daten angepasst, wobei der Fokus auf Verbindungen zwischen Neuronen im C.Pe lag. Wir variierten sowohl die Stärke der inhibitorischen Synapsen als auch der Gap Junctions im G.Pe. Inhibition zwischen G.Pe Neuronen konnte das Netzwerk inklusive subthalamischem Kern und internem Teil des Gilobus Pallidus desynchronisieren, wohingegen Gap Junctions dies nur bei sehr kleine Leitfähigkeiten taten. Bei höheren Leitfähigkeiten synchronisierten Gap Junctions das gesamte Netzwerk. Erwähnenswert ist, dass die Synchronisation verstärkt im Beta-Band zu finden war, und weniger in anderen Frequenzbändern. Wir fanden den beschriebenen Effekt ebenso in einem kleineren Modell mit weniger Neuronen, und er trat bei zwei verschiedenen GPe Neuronen-Modellen auf. Auch die genaue Architektur der verschiedenen Verbindungen hatte nur einen zweitrangigen Einfluss auf das Entstehen von Synchronität. Wir schlussfolgern daher, dass pallidale Verbindungen für synchrone neuronale Aktivität in den Basalganglien besonders wichtig sind - obwohl die Anzahl dieser Verbindungen vermutlich sehr klein ist.

Schließlich untersuchen wir in Kapitel 5 Korrelationen zwischen Aktivität im Ausgangsstadium der Basalganglien und im Eingangsstadium des Thalamus. Diese Korrelationen sind von besonderer Relevanz, da sie Einsichten in die physiologische Aufgabe der Aktivität in den Basalganglien ermöglichen, indem wir die Wirkung 
dieser Aktivität auf den Thalamus studieren. Während die Interaktionen zwischen einzelnen Zellen sehr schwach waren, sogar während Bewegungen, fanden wir starke Korrelationen zwischen lokalen Feldpotentialen der beiden Strukturen. Die hohen Korrelationen der lokalen Feldpotentiale konnten nur sehr eingeschränkt durch einen direkten, linearen Einfluss des Kortex auf beide Areale erklärt werden. Jedoch konnten wir geringe, aber signifikante Kohärenz im Beta-Band feststellen, als wir die Korrelationen der Aktivität von vielen Neuronen-Paaren mittelten. Folglich wäre es möglich, dass Informationen von den Basalganglien zum Thalamus über die Kooperation einer großen Anzahl an Neuronen übertragen werden, was sich auch in den lokalen Feldpotentialen widerspiegelt. In pathologischen Zuständen wie PD könnte diese Kooperation gestört sein.

Diese Arbeit hinterlässt viele offene Fragen. Die wichtigste Frage betrifft die Funktionalität der Gap Junctions im C.Pe, und das Maß dieser Funktionalität. Ein Nachweis der Funktionalität ist nur durch gleichzeitige, gepaarte intrazelluläre Ableitungen von GPe Neuronen möglich, und wird erschwert durch die geschätzt geringe Anzahl an Gap Junctions im C.Pe. Trotzclem könnten Experimente durchgeführt werden, die den möglichen Einfluss solcher Gap Junctions prüfen, auch bei ausstehendem formellen Nachweis ihrer Funktionalität. Zum Beispiel prognostizieren wir, dass Mäuse ohne das Connexin-36-Gen nach Administration von 6-Hydroxydopamin mehr Synchronität in den Basalganglien zeigen als naive Mäuse nach der gleichen Administration. Eine genauere Beschreibung von Anatomie und Physiologie der Basalganglien kann uns helfen, Informationsverarbeitung in subkortikalen Strukturen besser zu verstehen, und könnte letztendlich zu verbesserten Therapien von Störungen der Basalganglien, wie der Parkinson-Krankheit, führen. 



\section{Publications}

\section{Journal Publications}

B. C. Schwab, R. J. A. van Wezel, and S. A. van Cills:

Sparse pallidal connectivity shapes synchrony in a network model of the basal ganglia.

Submitted, March 2016

M. A. J. Lourens, B. C. Schwab, J. Nirody, H. G. E. Meijer, and S. A. Van Cills:

Exploiting pallidal plasticity for stimulation in Parkinson's disease.

Journal of Neural Engineering, 12(2), April 2015

B. C. Schwab, T. Heida, Y. Zhao, S. A. van Gils, and R. J. A. van Wezel:

Pallidal gap junctions - Triggers of synchrony in Parkinson's disease?

Movement Disorders, 29(12), pp. 1486-1494, October 2014

B. C. Schwab, T. Heida, Y. Zhao, E. Marani, S. A. van Cils, and R. J. A. van Wezel:

Synchrony in Parkinson's disease: Importance of intrinsic properties of the external globus pallidus.

Frontiers in Systems Neuroscience, 7(60), October 2013

B. C. Schwab, G. Seemann, R. A. Lasher, N. S. Torres, E. M. Wülfers, M. Arp, E. D. Carruth, J. H. B. Bridge, and F. B. Sachse:

Quantitative analysis of cardiac tissue including fibroblasts using three-dimensional confocal microscopy and image reconstruction: Towards a basis for electrophysiological modeling.

IEEE Transactions on Medical Imaging, 32(5), pp. 862-872, May 2013 


\section{Conference Contributions}

B. C. Schwab, R. J. A. van Wezel, and S. A. van Cills:

Synchronization of the Parkinsonian globus pallidus by neural gap junctions.

IBRO World Congress, Rio de Janeiro, Brazil, 2015 (Talk)

B. C. Schwab, H. G. E. Meijer, R. J. A. van Wezel, and S. A. van Cills:

Gap junctions as modulators of synchrony in Parkinson's disease.

Society for Neuroscience Annual Meeting (SfN), Washington DC, USA, 2014 (Talk)

B. C. Schwab, H. G. E. Meijer, R. J. A. van Wezel, and S. A. van Cills:

Synchronization of the Parkinsonian globus pallidus by gap junctions.

Computational Neuroscience, Québec City, Canada, 2014 (Talk)

B. C. Schwab, H. G. E. Meijer, R. J. A. van Wezel, and S. A. van Cills:

Basal ganglia dynamics assuming pallidal gap junctions.

CNS Workshop: Dynamics of Disease States, Québec City, Canada, 2014 (Talk)

B. C. Schwab, S. A. van Cils, Y. Zhao, T. Heida, and R. J. A. van Wezel:

Possible roles of neural gap junctions in network activity changes in Parkinson's disease.

International Basal Ganglia Society Meeting, Eilat, Israel, 2013 (Poster)

B. C. Schwab, R. J. A. van Wezel, T. Heida, and S. A. van Gils:

Possible roles of neural gap junctions in Parkinson's disease pathology.

4th Dutch Biomedical Engineering Conference, Egmond aan Zee, The Netherlands, 2013 (Talk)

E. D. Carruth, R. A. Lasher, B. Schwab, F. B. Sachse, and R. W. Hitchcock:

Three-dimensional modeling and quantitative analysis of protein distributions in cardiac tissue.

7th Annual Mountain West Biomedical Engineering Conference, 2011 (Poster) 


\section{Acknowledgements}

Acknowledgements appear in print version only. 


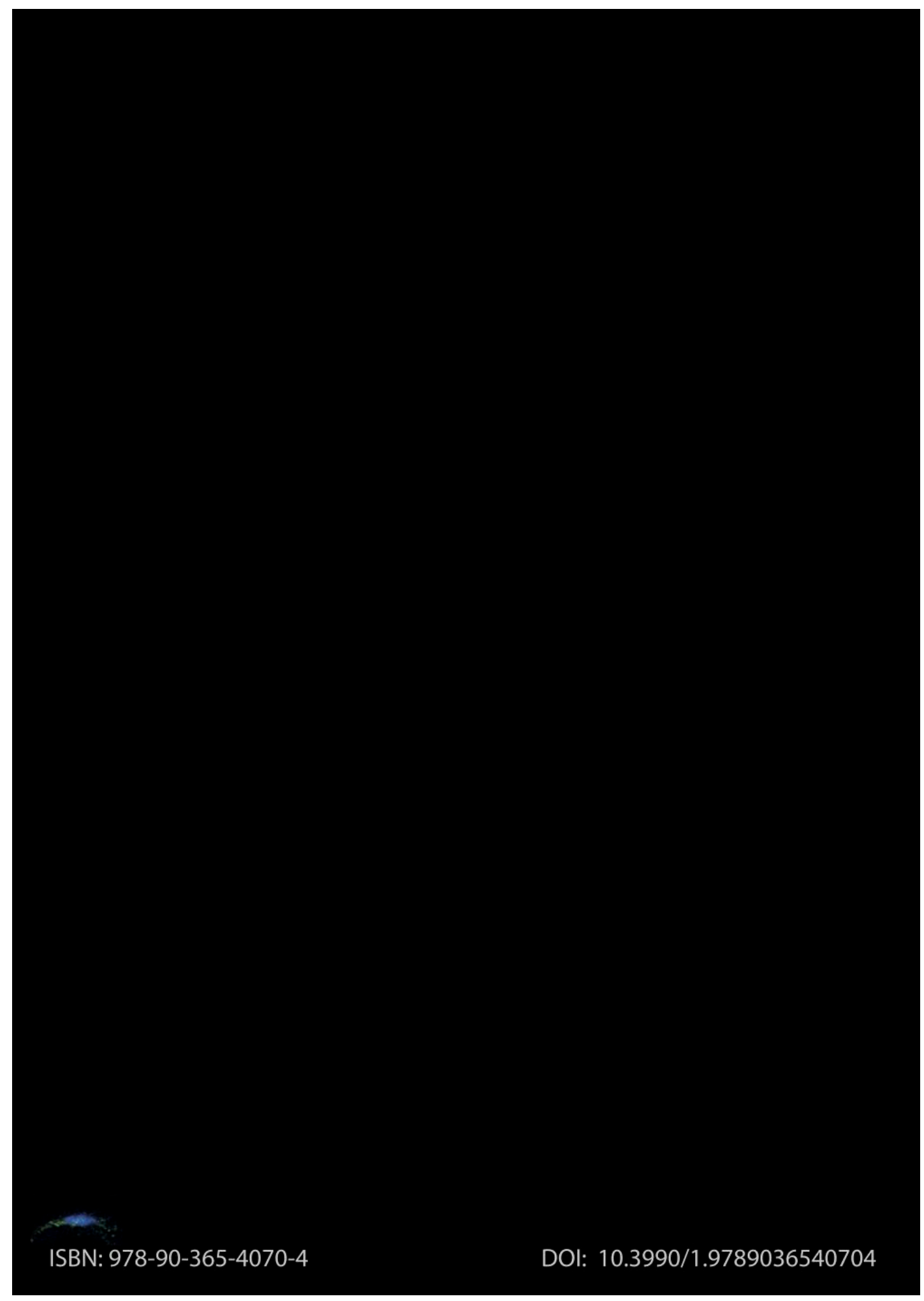

